\title{
Lithospheric Structure of the Hikurangi Subduction Margin
}

\author{
Herath Mudiyanselge \\ Manusha Pasan Sanjaya Herath
}

\author{
A thesis \\ submitted to the Victoria University of Wellington \\ in fulfilment of the \\ requirements for the degree of \\ Doctor of Philosophy in Geophysics
}

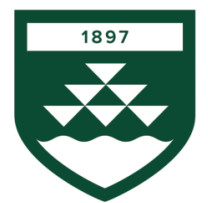

VICTORIA UNIVERSITY OF

WELLINGTON

TE HERENGA WAKA

NEW ZEALAND

School of Geography, Environment and Earth Sciences

Victoria University of Wellington

Wellington, New Zealand

May 2021 



\section{Abstract}

In this thesis, controlled-source seismic data acquired during two regional-scale experiments are analysed to determine the offshore lithospheric structure at the Hikurangi subduction margin in New Zealand. Subduction of the 120 Myr old Hikurangi Plateau occurs beneath the east coast of North Island, New Zealand. Because the plateau is an oceanic large igneous province, where the crustal thickness is about $50 \%$ greater than normal oceanic crust, there are different dynamics and seismicity patterns compared to the subduction of a regular oceanic crust. On interseismic time-scales, the plate interface in the south is locked down to depths of 30 $\mathrm{km}$ and experiences deep (30-45 km) slow-slip events (SSEs). In contrast, the plate interface is creeping in the north and experiences shallow $(5-10 \mathrm{~km})$ SSEs. It is important to understand the seismic velocity structure from the upper crust down to the base of the lithosphere in order to gain insights into the observed variations in subduction-thrust slip behaviour, SSEs and the structure of an oceanic plateau in general. The thesis consists of three projects, each focusing on different aspects of the lithosphere at the Hikurangi subduction margin.

Project one investigates the crustal and upper mantle structure of the subducting Hikurangi Plateau at the southern Hikurangi margin. In this study, onshore-offshore seismic data acquired during the Seismic Array HiKurangi Experiment (SAHKE) are used. By forward-model raytracing the travel-times of observed refractions and wide-angle reflections in common receiver gathers, the Hikurangi Plateau crustal thickness is estimated to be $12 \pm 1 \mathrm{~km}$. A $\sim 10 \%$ reduction in P-wave-speeds in the Hikurangi Plateau crust beneath the trough is observed. Refractions provide evidence for two upper mantle layers: an upper layer with regular upper mantle P-wave-speeds $(8.0 \pm 0.2 \mathrm{~km} / \mathrm{s})$; and a deeper layer with a high P-wave-speed $(8.7 \pm 0.2 \mathrm{~km} / \mathrm{s})$ at a 
depth of $50 \pm 2 \mathrm{~km}$ beneath the Hikurangi trough. Similarly fast upper mantle P-wave speeds are reported along margin-parallel azimuths under the North Island, about $100 \mathrm{~km}$ down-dip of the subduction zone at depths of $\sim 8-10 \mathrm{~km}$ from the Moho suggesting that the upper mantle of the Hikurangi Plateau is characterised by anomalously high P-wave-speeds along all azimuths. A velocity reduction of $\sim 10 \%$, similar to that in the crust, is deduced to extend down to $25 \pm 2 \mathrm{~km}$ in the upper mantle beneath the trough, as a result of the formation of a low-velocity zone in the faster upper mantle layer. It is proposed that this is due to the serpentinisation of mantle peridotite by hydration through bending-induced normal faults and/or due to crack porosity introduced by thermal cracking, further enhanced by bending-related faulting. This implies that the "regular mantle" $\left(\mathrm{V}_{\mathrm{P}} \sim 8 \mathrm{~km} / \mathrm{s}\right)$ is not regular, but rather the faster upper mantle has mechanically bent, fractured and altered. The onset of seismicity in the lower band of the double seismic zone and high upper mantle $V_{P}$ under the North Island is observed at depths of $\sim 50 \mathrm{~km}$. This is consistent with the hypothesis that the lower band of earthquakes in a double seismic zone is due to antigorite dehydration processes, a hydrous mineral in the low-velocity zone in the upper mantle beneath the trough. Despite the differences in crustal thickness and high upper mantle P-wave speeds, subduction-related upper mantle hydration and dehydration are analogous with other margins where regular oceanic crust is subducting.

The second project is focused on a series of long-offset, late-arriving wide-angle seismic reflections observed in the onshore-offshore common receiver gathers from the first project. Results from modelling these wide-angle reflections using forward-model raytracing, amplitude versus offset modelling and synthetic waveform modelling, are consistent with a series of reflective horizons approaching sub-lithospheric depths of the subducting Pacific Plate. A $\sim 3-5 \mathrm{~km}$ thick, azimuthally anisotropic layer with a $\mathrm{P}$-wave anisotropy of $13-15 \%$ is proposed to exist at a depth of $70 \mathrm{~km}$. A $5 \mathrm{~km}$ thick layer with low P-wave velocity $(7.6 \mathrm{~km} / \mathrm{s})$ and a high $\mathrm{V}_{\mathrm{P}} / \mathrm{V}_{\mathrm{S}}$ ratio $(>>2.5)$ is then required below the anisotropic layer. It is followed by another $\sim 3-5$ $\mathrm{km}$ thick layer with slightly lower $(7.4 \mathrm{~km} / \mathrm{s})$ or higher $(7.8 \mathrm{~km} / \mathrm{s})$ P-wave velocity and a regular $\mathrm{V}_{\mathrm{P}} / \mathrm{V}_{\mathrm{S}}$ ratio $(\sim 1.85)$. The higher $\mathrm{V}_{\mathrm{P}} / \mathrm{V}_{\mathrm{S}}$ ratio in the upper layer indicates that it contains either melt or volatiles, whereas the relatively low $V_{P} / V_{S}$ ratio in the 
lower layer may indicate a relatively lower fluid content. These two layers comprise a composite low $\mathrm{V}_{\mathrm{P}}$ layer with a thickness of $\sim 8-10 \mathrm{~km}$ beneath the anisotropic layer, and is interpreted to be the lithosphere-asthenosphere boundary (LAB) channel. It is consistent with the down-dip projected depths of the LAB channel found from an earlier study. The most prominent discovery here is the azimuthally anisotropic layer whose fast azimuth is subparallel to the direction of absolute plate motion (perpendicular to the margin). Strong shearing occurring at the LAB channel due to the differential movement of the lithosphere on top of the asthenosphere is suggested to give the preferential alignment of olivine crystals along the direction of maximum finite shear strain and produce the observed azimuthal anisotropy. Results from this study show, therefore, that it is not a single low-velocity channel that makes up the LAB, but a series of layers that make up an LAB zone. In addition, the study highlights the key role that wide-angle reflections from controlled-sources can play in investigating the fine-scale structure of the LAB boundary zone due to the short wavelengths and the generation of enhanced amplitudes when the reflections approach the critical angles $\left(\sim 55^{\circ}\right)$.

The primary objective of the third research project is to estimate the $V_{\mathrm{P}} / \mathrm{V}_{\mathrm{S}}$ ratio of the Hikurangi margin forearc using mode-converted seismic phases from airgun shots recorded by an array of multi-component ocean bottom seismographs (OBS) deployed as a part of the Seismogenesis at Hikurangi Integrated Research Experiment (SHIRE). PPS mode-conversions at the sediment-basement interface and the top of the subducting crust are identified. Estimated average $V_{P} / V_{S}$ ratios for the topmost sediments range from $2.5 \pm 1.0$ to $6.0 \pm 2.5$ and are consistent with water-saturated, unconsolidated sediments. Average $\mathrm{V}_{\mathrm{P}} / \mathrm{V}_{\mathrm{S}}$ ratios for the entire column of sediments and sedimentary rocks above the subducting crust range from $\sim 1.55 \pm 0.08$ to 2.20 \pm 0.08 . Low-average $\mathrm{V}_{\mathrm{P}} / \mathrm{V}_{\mathrm{S}}$ ratios between $\sim 1.55 \pm 0.08$ and $\sim 1.78 \pm 0.12$ are estimated for a region of higher sediment thickness in the southern Hikurangi margin. The thick sediments may result in a higher degree of compaction. The low $\mathrm{V}_{\mathrm{P}} / \mathrm{V}_{\mathrm{S}}$ ratios are also coincident with the offshore extension of the Pahau Torlesse Terrane which consists mainly of low-porosity, highly compacted, Cretaceous greywackes. In contrast, high $\mathrm{V}_{\mathrm{P}} / \mathrm{V}_{\mathrm{S}}$ ratios between $\sim 1.85 \pm 0.10$ and $2.22 \pm 0.08$ are observed in regions with lower sediment thickness, which may reflect effects of lower 
degree of compaction and lithology. Furthermore, the average $\mathrm{V}_{\mathrm{P}} / \mathrm{V}_{\mathrm{S}}$ of the rocks and sediments above the subducting crust show a weak correlation with the slip-rate deficits on the subduction thrust. Shear-wave splitting results indicate an anisotropy of $\sim 3.5 \%$ localised in the top layer $(\sim 1-2 \mathrm{~km})$ of sediments beneath the seafloor. Fast polarisation directions are oriented perpendicular to the plate interface contours, suggesting stress-aligned, fluid-filled cracks.

The work presented in this thesis provides constraints on the lithospheric structure of the Hikurangi subduction margin, from the upper plate down to the base of the lithosphere, using controlled-source seismology. The results provide insights on the physical properties of the materials and their association with geodynamic processes. The outcomes of this thesis advance our knowledge of the Hikurangi subduction margin and contribute to our knowledge of plate tectonics in general. 


\section{Acknowledgements}

I am lucky and privileged to have been supported by many people during my PhD. First and foremost, my sincerest thanks go to my supervisors, Tim Stern and Martha Savage, for their enormous support and guidance since the very first day of my arrival in Wellington. Tim and Martha have been wonderful mentors who always had an open door whenever I came across any issue. Tim's optimism and consistence encouragement was very instrumental in transforming my efforts into a successful project with good outcomes. Martha's unwavering support and guidance for research helped my PhD life flow smoothly.

I am also grateful to my supervisors from GNS Science, Dan Bassett and Stuart Henrys who provided valuable insights, ideas and guidance which helped increase the quality of the research. I sincerely acknowledge Carolyn Boulton, Simon Lamb, Rupert Sutherland, John Townend, Calum Chamberlain and Finn Illsley-Kemp for teaching the courses that I attended during the $\mathrm{PhD}$ and for being there to help and discuss whenever I ran into problems.

Huw Horgan from Victoria University of Wellington, Ingo Pecher from the University of Auckland and Donna Shillington from the Northern Arizona University are acknowledged for their encouraging comments, constructive criticism and suggestions in revising the thesis and in preparing manuscripts from the unpublished project chapters.

I am also thankful to Adrian Benson and Aleksandr Beliaev for helping me with technical issues and guiding me towards feasible and future-proof paths. Adrian has been wonderful to share his Geophysics knowledge with me when I was in the first stages of my PhD.

I am thankful to my officemates and colleagues from the Geophysics group and 
Antarctic Research Center: Bryant Chow, Hubert Zal, Dominic Evanzia, Konstantinos Michailos, Abhijith Venugopal, Hamish Hirschberg, Kenny Graham, Chet Hopp, Weiwei Wang, Katie Woods, Wei Ji Leong, Jamey Stutz, Steven Kessler, Andrea Perez, El Mestel, Levan Tielidze, Jesse Kearse, Steven Sewell for all the help, discussions and the occasional banter throughout my PhD journey.

I would like to acknowledge the support of Wanda Stratford, Katrina Jacobs, Rory Hart, Dan Barker, Richard Kellet, Jenny Black, Brook Tozer, and the SHIRE and NZ3D field teams for the help and support during fieldwork. Thank you Wanda and Brook for the help, ideas and suggestions that you provided during my PhD.

I am also thankful to the New Zealand Ministry of Business, Innovation and Employment (MBIE) and GNS Science for providing me a scholarship to support with my PhD and also the Doctoral Submission Scholarship of the Victoria University of Wellington, which helped me immensely during the last few months of the PhD. Miranda Voke, Emily Brook, Monica Hanson, Steff Marinus and Minda Goncalves from SGEES office and Patricia Stein from the Faculty of Science are thanked for their support.

My heartfelt thanks go to Karen Brown and Tim for welcoming and hosting me when I first arrived in Wellington.

I am very much indebted to my parents Sujatha and Tikiri for their never-ending love, affection and guidance for a career in science and for all the support and encouragement during the PhD. I am thankful to my sister Hansika and brother-in law Janaka for their support throughout, and their little one Hiruka who brought many smiles during the PhD. Thank you my parents-in-law Kumari and Upali, and brother-in-law Naveen for their support and encouragement.

I have been so lucky to have married a wonderful woman, Bimaya, who kept her career aside and assisted me immensely during my $\mathrm{PhD}$. Thanks very much for being a caring and loving best friend. 


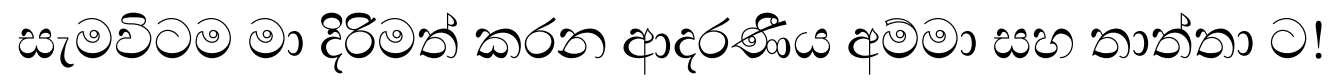

To my beloved mom and dad, for your endless encouragement! 



\section{Contents}

$\begin{array}{ll}\text { Abstract } & \text { i }\end{array}$

$\begin{array}{ll}\text { Acknowledgements } & \text { v }\end{array}$

1 Introduction 1

1.1 Context and Rationale . . . . . . . . . . . . . . . . . . . . 1

1.2 Thesis Structure . . . . . . . . . . . . . . . . . . . . . . 4

1.3 Previous Studies on the Lithospheric Structure at the Hikurangi Margin 7

1.3.1 Hikurangi Margin Forearc . . . . . . . . . . . . . . 8

1.3.2 Hikurangi Plateau . . . . . . . . . . . . . . . . . . 9

2 Theory and Methods 13

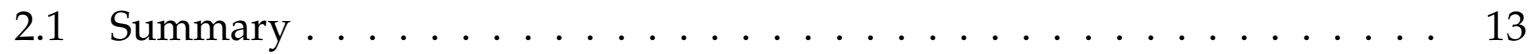

2.2 Seismic Waves and Rays . . . . . . . . . . . . . . . . . . . . . 13

2.3 Controlled-source Seismology . . . . . . . . . . . . . . . . . 15

2.3.1 Multichannel Seismic Reflection Surveying . . . . . . . . . . . . . 16

2.3.2 Wide-angle Reflection and Refraction Seismic Surveying . . . . . 17

2.4 Processing Wide-angle Seismic Data . . . . . . . . . . . . . . . 20

2.4.1 Common Receiver Gathers . . . . . . . . . . . . . . . . . . 20

2.4 .2 Improving Signal-to-noise Ratio . . . . . . . . . . . . . . . 22

2.5 Phase Identification . . . . . . . . . . . . . . . . . . . . . . . 23

2.6 Travel-time Modelling . . . . . . . . . . . . . . . . . . . 24

2.6.1 Raytrace Modelling . . . . . . . . . . . . . . 25

2.6 .2 Uncertainties . . . . . . . . . . . . . . . 27

2.7 Seismic Anisotropy . . . . . . . . . . . . . . . . . . . . . 28

2.7.1 Shear-wave Splitting . . . . . . . . . . . . . . . . 31

2.8 Synthetic Waveform Modelling . . . . . . . . . . . . . . . . . . . 32 
2.8.1 Spectral-element Method . . . . . . . . . . . . . . . . . 34

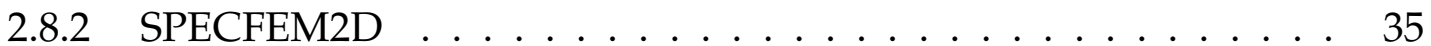

3 Hydration of the Crust and Upper Mantle of the Hikurangi Plateau as it Subducts at the Southern Hikurangi Margin 39

Abstract . . . . . . . . . . . . . . . . . . . . . . . . . . . . . . . 39

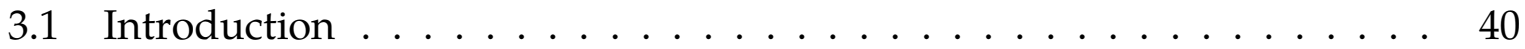

3.2 Hikurangi Subduction Margin . . . . . . . . . . . . . . . . . . . 41

3.3 Data . . . . . . . . . . . . . . . . . . . . . . 43

3.4 Velocity Modelling . . . . . . . . . . . . . . . . . . . . 44

3.4.1 Onshore-offshore Common Receiver Gathers . . . . . . . . . . . . 44

3.4 .2 Initial Models . . . . . . . . . . . . . . . . . . . . . 50

3.4.3 Forward-model Raytracing . . . . . . . . . . . . . . . . . 51

3.4.4 Model Parameter Uncertainties . . . . . . . . . . . . . . . . . . 52

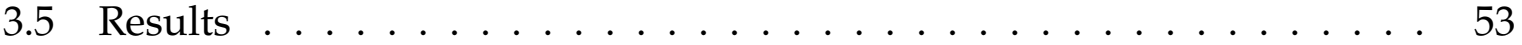

3.5.1 Crustal Structure of Overriding Australian Plate . . . . . . . . 54

3.5.2 Crustal Structure of Subducting Pacific Plate . . . . . . . . . 57

3.5.3 Upper Mantle Structure of Subducting Pacific Plate . . . . . . . 58

3.6 Discussion . . . . . . . . . . . . . . . . . . . . . . . . . . . . . . . . . . 59

3.6.1 Oceanic Crustal Thickness of Hikurangi Plateau . . . . . . . . . 59

3.6.2 High Upper Mantle P-wave-speeds of Hikurangi Plateau . . . . . 60

3.6.3 Hydration and Dehydration of the Subducting Slab . . . . . . . 61

3.6.4 Deep-lithospheric Reflections from the Pacific Plate . . . . . . . . 64

3.7 Conclusions . . . . . . . . . . . . . . . . . . . . . 66

4 Evidence for a Lithosphere-Asthenosphere Boundary Zone of the Pacific Plate $\begin{array}{ll}\text { at the Southern Hikurangi Margin } & 67\end{array}$ Abstract . . . . . . . . . . . . . . . . . . . . . . . . . . 67

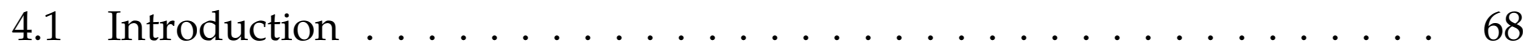

4.2 Structure of the Pacific Plate at Hikurangi margin . . . . . . . . . 69

4.3 Data . . . . . . . . . . . . . . . . . . . . . . . . . . 69

4.4 Wide-angle Reflections in the Onshore-offshore Data . . . . . . . . . 70

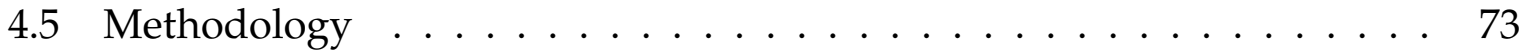

4.6 Depths of Reflectors and Interval Velocities _ . . . . . . . . . . . 77 


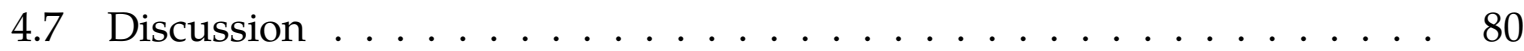

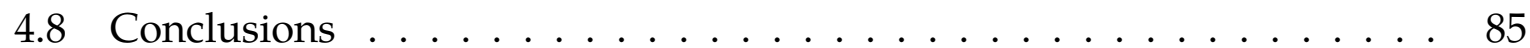

$5 \quad \mathrm{~V}_{\mathrm{P}} / \mathrm{V}_{\mathrm{S}}$ Ratio, Material and Slip-rate Deficit Variations along Hikurangi Margin Forearc $\quad 87$

Abstract . . . . . . . . . . . . . . . . . . . . . . . 8 87

5.1 Introduction $\ldots \ldots \ldots \ldots \ldots \ldots \ldots \ldots \ldots$

5.2 Tectonic Setting $\ldots \ldots \ldots \ldots \ldots$

5.3 Data . . . . . . . . . . . . . . . . . . 92

5.3 .1 OBS Gathers . . . . . . . . . . . . . . . . . . . . . . . . 93

5.4 Orienting OBS Horizontal Components $\ldots \ldots \ldots \ldots$

5.4 .1 Hodogram Analysis . . . . . . . . . . . . . . . . . . . 94

5.4 .2 Polarisation Analysis . . . . . . . . . . . . . . . . . . . . 94

5.4 .3 Resolving Ambiguity . . . . . . . . . . . . . . . . . 97

5.4.4 Rotating to Radial and Transverse Components _ . . . . . . . . 98

5.5 Processing OBS gathers . . . . . . . . . . . . . . . . . . 98

5.6 Identification of Mode-converted Phases . . . . . . . . . . . . . . . 98

5.6.1 Polarisation Angles and Rectilinearity . . . . . . . . . . . . 100

5.6 .2 Linear Moveout . . . . . . . . . . . . . . . . . . . . . . . . 105

5.6.3 Comparison with Hydrophone Component . . . . . . . . . . . 105

5.7 Identified Mode-converted Phases ． . . . . . . . . . . . . . . . . . 107

5.7.1 PPS Mode-converted Phases ． . . . . . . . . . . . . . . . 107

5.8 Average $\mathrm{V}_{\mathrm{P}} / \mathrm{V}_{\mathrm{S}}$ Estimation from PPS Mode-converted Phases . . . . . . 109

5.8 .1 Calculating $\Delta \mathrm{T} \ldots \ldots \ldots \ldots \ldots \ldots$

5.8.2 Calculating $\mathrm{T}_{\mathrm{P}}$ and $\mathrm{T}_{\mathrm{Psf}} \ldots \ldots \ldots \ldots \ldots \ldots$

5.9 Shear-wave Splitting . . . . . . . . . . . . . . . . . . . . . 117

5.10 Results and Discussion . . . . . . . . . . . . . . . . . . 118

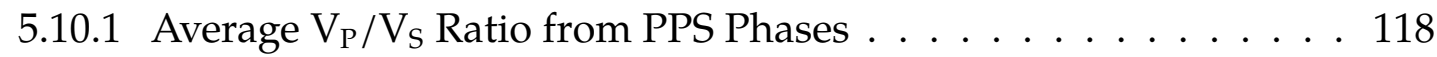

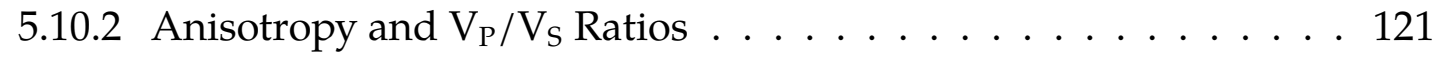

5.10.3 Average $V_{\mathrm{P}} / \mathrm{V}_{\mathrm{S}}$ Ratio, Lithology, Sedimentary Thickness and Sliprate Deficit Variations . . . . . . . . . . . . . . . . 122

5.11 Conclusions . . . . . . . . . . . . . . . . . . . . . 124

$\begin{array}{lll}6 & \text { Thesis Summary } & 127\end{array}$ 
6.1 Synthesis . . . . . . . . . . . . . . . . . . . . . 127

6.2 Research Questions . . . . . . . . . . . . . . . . . . . . . . . . . . . 129

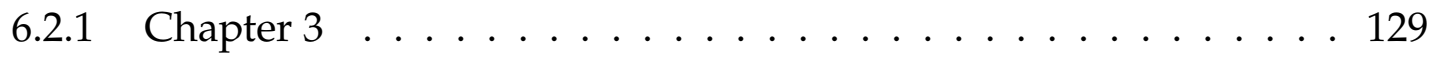

6.2 .2 Chapter $4 \ldots \ldots \ldots \ldots \ldots 131$

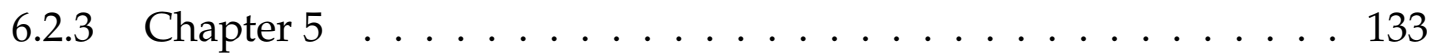

6.3 Concluding Remarks . . . . . . . . . . . . . . . . . . 135

$\begin{array}{ll}\text { Appendices } & 137\end{array}$

$\begin{array}{lll}\text { A Supplementary Material for Chapter 3 } & 139\end{array}$

A.1 Pre-modelling Considerations . . . . . . . . . . . . . . . . . . . . . 141

A.1.1 Defining the Transects . . . . . . . . . . . . . . . . . . . 141

A.1.2 Projection of SAHKE01 Airgun Shots . . . . . . . . . . . . 141

B Supplementary Material for Chapter 4 171

$\begin{array}{lll}\text { C Supplementary Material for Chapter } 5 & 183\end{array}$ 


\section{List of Figures}

1.1 Tectonic setting around New Zealand . . . . . . . . . . . . . . . . 2

1.2 Tectonic setting at the Hikurangi subduction margin . . . . . . . . . . . 3

2.1 Schematic diagram of a wavefront and a raypath . . . . . . . . . . . 14

2.2 Partitioning of an obliquely incident seismic raypath at an interface . . . 15

2.3 Schematic of an on-land seismic experiment . . . . . . . . . . . 17

2.4 Schematic of an onshore-offshore acquisition geometry . . . . . . . . . 19

2.5 Schematic of an ocean-bottom acquisition geometry . . . . . . . . . . . 21

2.6 RAYINVR model parametrisation . . . . . . . . . . . . . . . . . 27

$2.7 \quad \mathrm{P}$ - and S-wave velocities along crystallographic directions of olivine . . . 32

2.8 Schematic of shear-wave splitting . . . . . . . . . . . . . . . 33

3.1 Map of study area . . . . . . . . . . . . . . . . . . . 45

3.2 Common receiver gather at PEG3 from PEGASUS23 airgun shots . . . . 46

3.3 Common receiver gather at PEG6 from PEGASUS25 airgun shots . . . . 47

3.4 Common receiver gather at MRZ from SAHKE01 airgun shots . . . . . . 48

3.5 Common receiver gather at BFZ from SAHKE01 airgun shots . . . . . . . 49

3.6 Final velocity models along PEGASUS23 and PEGASUS25 transects . . . 55

3.7 Perturbation, grid-search, sensitivity test results and ray density of turning waves in the Hikurangi Plateau crust . . . . . . . . . . . . . . 56

3.8 Conceptual model of the hydration of the Hikurangi Plateau beneath the trough and its dehydration down-dip of the subduction zone . . . . . . . 65

4.1 Map of the study area with the surrounding tectonic setting $\ldots$. . . . 71

4.2 Onshore-offshore common receiver gathers showing late-arriving, faroffset arrivals . . . . . . . . . . . . . . . . . 72

4.3 Raytrace model for the observed phases in the common receiver gather at BFZ with SAHKE01 airgun shots . . . . . . . . . . . . . . . 75 
4.4 Synthetic common receiver gather at BFZ station with SAHKE01 airgun

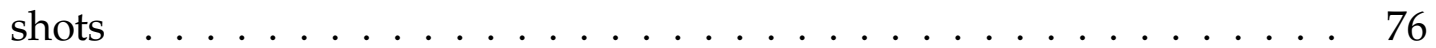

4.5 One-dimensional P-wave velocity models with different velocity contrasts and travel-times of the reflected phases . . . . . . . . 78

4.6 Relative amplitudes of $\mathrm{R}_{0}-\mathrm{R}_{3}$ wide-angle reflections $\ldots \ldots \ldots$

4.7 Reflection coefficients for $R_{0}-R_{3}$ from AVO modelling $\ldots \ldots \ldots$. . . . . 81

4.8 Schematic diagram of the lithosphere-asthenosphere boundary zone at the Hikurangi margin . . . . . . . . . . . . . . . . . . . . . . . . 82

5.1 Variation of Poisson's ratio with $\mathrm{V}_{\mathrm{P}} / \mathrm{V}_{\mathrm{S}} \ldots \ldots \ldots \ldots$

5.2 OBS locations along Hikurangi forearc . . . . . . . . . . . . . . . 91

5.3 JAMSTEC OBS instrumentation . . . . . . . . . . . . . . . . . . 93

5.4 Orientation of the components of the triaxial geophones of an OBS . . 95

5.5 Direct water arrival hodogram of the horizontal components at OBS $309 \quad 95$

5.6 Linear regression analysis of the direct water arrival at OBS $309 \ldots 6$

5.7 Polarisation angles from eigendecomposition of $\mathrm{H}_{1}$ and $\mathrm{H}_{2}$ covariance

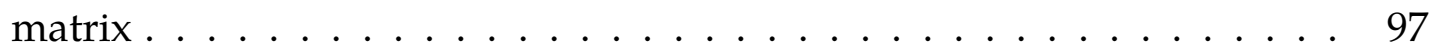

5.8 Schematic diagram for resolving OBS orientation ambiguity $\ldots$. . . . . 100

5.9 Hodograms of radial versus vertical components rotated using the two ambiguous angles . . . . . . . . . . . . . . . . . . 101

5.10 Hodograms of vertical, radial and transverse components at OBS 309 after correct rotation . . . . . . . . . . . . . . . . . . 102

5.11 Vertical, radial, transverse and hydrophone component gathers at OBS 309103

5.12 Zoomed vertical, radial, transverse and hydrophone component gathers at OBS $309 \ldots \ldots \ldots$. . . . . . . . . . . . . . . . . . . . 104

5.13 Schematic showing PSS, PPS and PSP mode-conversions . . . . . . . . 104

5.14 Polarisation angle and rectilinearity of arrivals at OBS 309 . . . . . . . 106

5.15 Vertical, radial, transverse and hydrophone component gathers at OBS 301108

5.16 Zoomed vertical, radial, transverse and hydrophone component gathers at OBS $301 \ldots \ldots \ldots$. . . . . . . . . . . . . . . . . . . . . . . . . . . . . . . . . . .

5.17 Vertical, radial, transverse and hydrophone component gathers at OBS 327110

5.18 Zoomed vertical, radial, transverse and hydrophone component gathers at OBS $327 \ldots \ldots \ldots \ldots$

5.19 Schematic diagram of PPS converted phases . . . . . . . . . . . . . . 112 
5.20 P- to S- transmission coefficients for incident plane waves . . . . . . . . . 113

5.21 Travel-time lags of $\mathrm{PP}_{\mathrm{o}} \mathrm{S}_{\mathrm{b}}$ phases $\ldots \ldots \ldots$. . . . . . . . . . . 113

5.22 Travel-time lags of $\mathrm{PP}_{\mathrm{o}} \mathrm{S}_{\mathrm{c}}$ phases . . . . . . . . . . . . . . . 114

5.23 Multichannel seismic reflection image along SHIRE03 OBS profile . . . . 115

5.24 $V_{\mathrm{P}}$ and average $\mathrm{V}_{\mathrm{P}} / \mathrm{V}_{\mathrm{S}}$ along Hikurangi margin forearc from PPS phases 116

5.25 Shear-wave splitting result at OBS 309 . . . . . . . . . . . . . . 119

$5.26 \mathrm{~V}_{\mathrm{P}} / \mathrm{V}_{\mathrm{S}}$ variation with porosity and clay content $\ldots \ldots \ldots . . \ldots 121$

5.27 Fast polarisation azimuths and delay times at OBS stations from shearwave splitting . . . . . . . . . . . . . . . . . 123

A.1 Map of high P-wave-speeds in the upper mantle of the Hikurangi Plateau 140

A.2 Projection of seismographs and airgun shots to the transect . . . . . . . 141

A.3 Apparent velocity difference when source-receiver azimuth is not inline with structural $\operatorname{dip} \ldots \ldots \ldots \ldots$. . . . . . . . . . . . . . . . . . . . . . . .

A.4 Common receiver gather at PEG1 from PEGASUS23 airgun shots . . . . 145

A.5 Common receiver gather at PEG2 from PEGASUS23 airgun shots . . . . 146

A.6 Common receiver gather at TMWZ from PEGASUS23 airgun shots . . 147

A.7 Common receiver gather at PEG4 from PEGASUS23 airgun shots . . . 148

A.8 Common receiver gather at PEG5 from PEGASUS23 airgun shots . . . . 149

A.9 Common receiver gather at PEG11 from PEGASUS23 airgun shots . . . . 150

A.10 Common receiver gather at PEG12 from PEGASUS23 airgun shots . . . . 151

A.11 Common receiver gather at TIWZ from PEGASUS23 airgun shots . . . 152

A.12 Common receiver gather at PEG13 from PEGASUS23 airgun shots . . . . 153

A.13 Common receiver gather at HOWZ from PEGASUS23 airgun shots . . . 154

A.14 Common receiver gather at MRZ from PEGASUS23 airgun shots . . . . 155

A.15 Common receiver gather at CPWZ from PEGASUS25 airgun shots . . . . 156

A.16 Common receiver gather at PEG7 from PEGASUS25 airgun shots . . . 157

A.17 Common receiver gather at BFZ from PEGASUS25 airgun shots . . . . 158

A.18 Common receiver gather at PEG8 from PEGASUS25 airgun shots . . . . 159

A.19 Common receiver gather at PRWZ from PEGASUS25 airgun shots . . . . 160

A.20 Common receiver gather at PEG3 from SAHKE01 airgun shots . . . . . . 161

A.21 Multichannel reflection image and stacking velocities along PEGASUS23

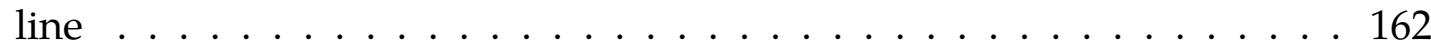


A.22 Multichannel reflection image and stacking velocities along PEGASUS25 line

A.23 Multichannel reflection image and stacking velocities along SAHKE01 line163

A.24 Perturbation test results for thickness, velocity of Australian Plate crust and the dip of the plate interface . . . . . . . . . . . . . 163

A.25 Three-parameter grid-search test for model parameter uncertainties . . . 164

A.26 Travel-time misfits with offset and histogram of travel-time misfits along PEGASUS23 transect . . . . . . . . . . . . . . . . . . . . . 164

A.27 Travel-time misfits with offset and histogram of travel-time misfits along PEGASUS25 transect . . . . . . . . . . . . . . . . . . . 165

A.28 Ray density along PEGASUS23 transect . . . . . . . . . . . . . . 165

A.29 Ray density along PEGASUS25 transect . . . . . . . . . . . . . . . 165

A.30 Plate interface comparison along PEGASUS23 transect . . . . . . . . . . 166

A.31 Plate interface comparison along PEGASUS25 transect . . . . . . . . . 167

A.32 Delay times for $P_{n 2}, R_{1}$ and $R_{2}$ phases . . . . . . . . . . . . 168

A.33 Comparison with the refraction profile of Chadwick [1997] . . . . . . . . 169

A.34 Comparison between curvatures of subducting slabs . . . . . . . . . . 169

B.1 Raytrace based test to assess the peg-leg multiple alternative for $R_{0}-R_{2}$

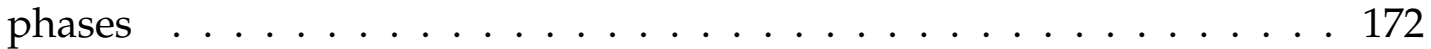

B.2 Raytrace based test to assess the peg-leg multiple alternative for $R_{0}-R_{2}$

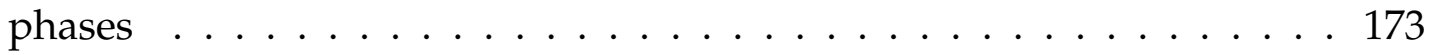

B.3 Particle motion of the $\mathrm{R}_{0}-\mathrm{R}_{3}$ phases to test $\mathrm{P}$ - to $\mathrm{S}$ - conversion and sideswipe alternatives . . . . . . . . . . . . . . . 174

B.4 Particle motion of the $\mathrm{R}_{0}-\mathrm{R}_{3}$ phases to test $\mathrm{P}$ - to $\mathrm{S}$ - conversion and sideswipe alternatives . . . . . . . . . . . . . . . 175

B.5 Water depth variation between original and projected SAHKE01 airgun

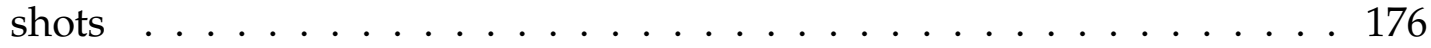

B.6 Water depth variation between original and projected SAHKE01 airgun

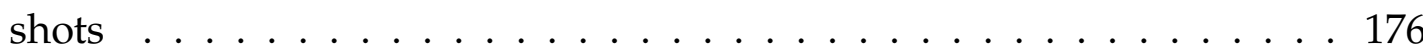

B.7 Raytrace model for the observed phases in the common receiver gather at MRZ station with SAHKE01 airgun shots . . . . . . . . . . . . . . . 177

B.8 Raytrace model for the observed phases in the common receiver gather at MRZ station with PEGASUS23 airgun shots . . . . . . . . . . . . 178 
B.9 Uncertainty estimate for the depth to $R_{0} \ldots \ldots \ldots \ldots$. . . . . . 178

B.10 Alternative raytrace model for the observed phases in the common receiver gather at BFZ station with SAHKE01 airgun shots . . . . . . . . 179

B.11 Synthetic common receiver gathers to estimate the uncertainties in velocities of the anisotropic layer . . . . . . . . . . . . . . . . . . 180

B.12 Synthetic common receiver gathers to estimate the uncertainty in the thickness of the anisotropic layer . . . . . . . . . . . . . . . 181

C.1 Vertical, radial, transverse and hydrophone component gathers at OBS 331184 



\section{List of Tables}

3.1 Observed seismic phases in the common receiver gathers along PEGASUS23 and PEGASUS25 transects . . . . . . . . . . . . . . . . 52

3.2 Details of perturbation and grid-search tests to estimate model parameter uncertainties . . . . . . . . . . . . . . . . . . . 54

5.1 Orientations of the horizontal components of OBS stations . . . . . . . 99

A.1 Seismograph location details . . . . . . . . . . . . . . . . . . . . . 144

B.1 Raytrace statistics for $R_{0}-R_{3}$ wide-angle reflections $\ldots \ldots \ldots$. . . . . 174

B.2 Model parameters for synthetic seismogram modelling . . . . . . . . 176 



\section{Chapter 1}

\section{Introduction}

\subsection{Context and Rationale}

New Zealand straddles the tectonic plate boundary between the Pacific and Australian Plates (Figure 1.1). The tectonic character of the plate boundary varies along its course through New Zealand. In the southwestern South Island of New Zealand is the Fiordland subduction margin, a recently initiated subduction zone system where the Australian Plate subducts beneath the Pacific Plate. It changes from subduction to a continental transform fault, the Alpine Fault further north in the South Island. The Alpine Fault continues up to the northern South Island until it translates into the Marlborough fault zone, which then merges with the Hikurangi subduction margin offshore of the eastern North Island of New Zealand.

The subducting Pacific Plate at the Hikurangi margin contains the relatively buoyant Hikurangi Plateau, an oceanic large igneous province [Coffin and Eldholm, 1994; Davy et al., 2008] (Figure 1.1). There are many along-strike variations in structure and character of the Hikurangi margin. Geodetic data reveals that the plate interface in the southern margin is locked (has a high slip-rate deficit) during interseismic time periods [Walcott, 1984] with deep slow-slip events (30-35 km) [Wallace et al., 2012a,b] (Figure 1.2). The locked subduction patch here has the potential to generate a megathrust earthquake [Wallace et al., 2004]. In the north, the subduction thrust is observed to be creeping, with shallow slow-slip events $(5-10 \mathrm{~km}$ ) [Wallace et al., 2004, 2012a,b] (Figure 1.2). The slow-slip events in the south are of long duration ( $\sim 1$ year) and with recurrence intervals of $\sim 5$ years. In the north, the slow-slip events are of short duration ( $<1$ month) and have recurrence intervals of $\sim 1-2$ years [Wallace, 


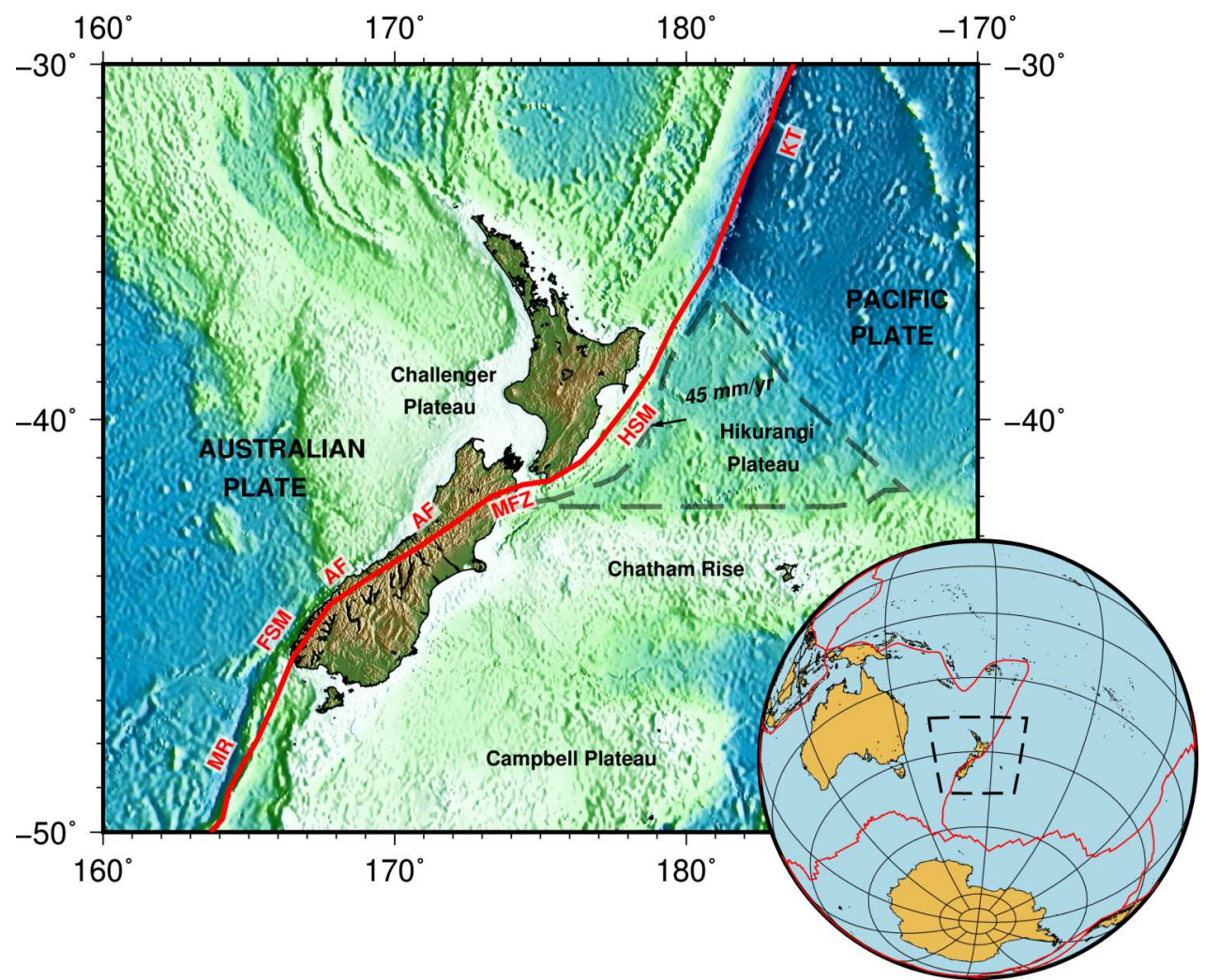

Figure 1.1 Tectonic setting around New Zealand. Red lines indicate active plate boundaries. HSMHikurangi subduction margin, AF-Alpine Fault, MFZ-Marlborough fault zone, FSM-Fiordland subduction margin, MR-McQuarie Ridge, KT-Kermadec trench. Black, dashed outline indicates the approximate extents of non-subducted portion of the Hikurangi Plateau. In the inset map of the globe, dashed back polygon indicates the extent of the main map, and red lines indicate the present-day tectonic plate boundaries.

2020]. The relative plate convergence obliquity changes from margin-perpendicular in the north to nearly margin-parallel in the south. The southern segment of the Hikurangi margin is characterised by frontal accretion whereas the northern segment is characterised by subducting seamounts and tectonic erosion [Wallace, 2020]. These variations in character make the Hikurangi margin an ideal location to understand the subduction dynamics associated with an oceanic plateau and the full spectrum of fault slip modes of a subduction thrust.

It has been challenging to explore the lithospheric structure offshore at the Hikurangi margin using passive-source seismic methods due to the lack of long-term offshore seismograph deployments. Recent offshore deployments of seismographs have been conducted but are confined to the northern part of the margin [e.g. Yarce et al., 2019; Zal et al., 2020]. Extensive seismic reflection surveys in the east coast for petroleum exploration have examined only the shallower part of the margin, at most down to the subduction interface [e.g. Barker et al., 2009; Bell et al., 2010]. 


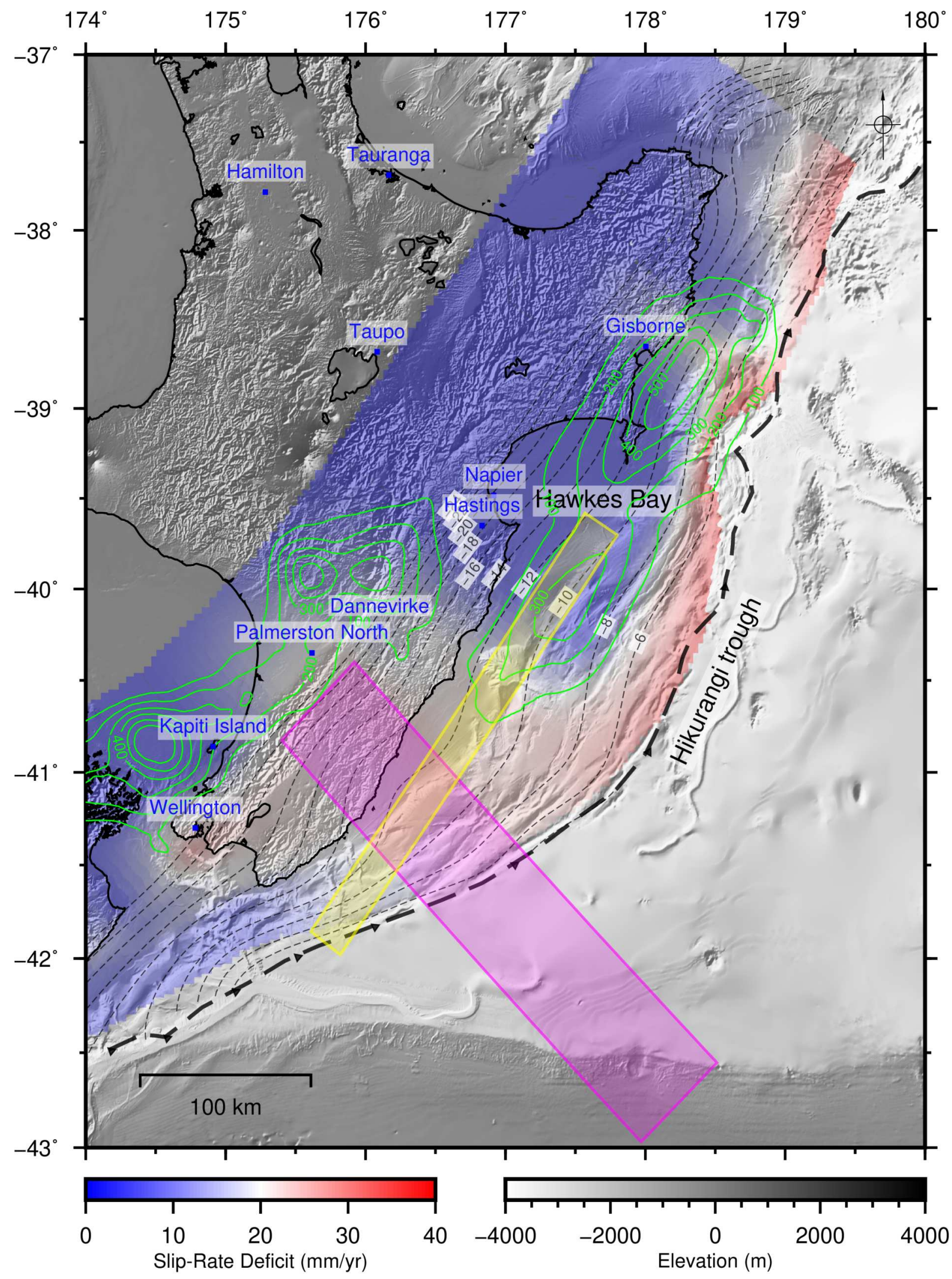

Figure 1.2 Tectonic setting at the Hikurangi subduction margin. Thick, black dashed line shows the Hikurangi trough. Thin, black dashed lines indicate the elevation of the plate interface from Williams et al. [2013]. Green lines indicate the slow-slip contours from Wallace et al. [2012a]. Slip-rate deficits are from Wallace et al. [2012b]. The region shaded in magenta is the study area of Chapters 3 and 4 . The region shaded in yellow is the study area of Chapter 5. 
As the depths to the subduction interface are shallower due to the buoyancy of the subducting Hikurangi Plateau and the presence of a large sub-aerial forearc region at the Hikurangi margin, a combination of controlled-source seismic methods can be used to image the full width of the subduction interface and the deeper velocity structure. Wide-angle modelling of ocean bottom seismograph data and near-offset modelling of multichannel seismic data can be performed in the submerged portion of the forearc while wide-angle modelling of onshore-offshore seismic data can be performed in the sub-aerial portion of the forearc.

Taking these favourable conditions for controlled-source seismology into account, two major controlled-source seismic experiments were conducted in New Zealand focusing on imaging the subsurface velocity structure of the Hikurangi subduction margin. Seismic Array HiKurangi Experiment (SAHKE) was conducted in 2009-2010 in the southern Hikurangi margin. Seismogenesis at Hikurangi Integrated Research Experiment (SHIRE) was conducted in 2017-2019 focusing on the northern Hikurangi margin where slow-slip events are observed and along the strike of the margin. In this thesis, controlled-source seismic data acquired in these two experiments are used to constrain the lithospheric structure at the Hikurangi subduction margin.

The overall objectives of the thesis are: constraining the crustal and upper mantle structure of the Hikurangi Plateau in the southern Hikurangi margin using onshore-offshore, controlled-source seismic data (i.e. offshore airgun shots recorded by onshore seismographs) acquired during SAHKE (magenta shaded region in Figure 1.2) and estimating the $V_{P} / V_{S}$ ratio of the upper plate (i.e. accretionary wedge) in the southern and central parts of the Hikurangi margin (yellow shaded region in Figure 1.2) using ocean bottom seismic data from SHIRE (i.e. airgun shots recorded by seismographs at the ocean bottom).

\subsection{Thesis Structure}

The thesis consists of 6 chapters.

\section{Chapter 1 - Introduction}

Chapter 1 provides an introduction to the thesis and an overview of the lithospheric structure at the Hikurangi subduction margin from previous studies. It also poses the 
research questions and the hypotheses to be tested in the research projects presented in the thesis.

\section{Chapter 2 - Theory and Methods}

Chapter 2 provides the theoretical and methodological framework relevant to the scope of this thesis. It provides an overview of seismic wave and ray theory, controlled-source seismology, controlled-source seismic survey geometries used in this thesis, modelling of controlled-source seismic data, seismic anisotropy and synthetic seismogram generation.

\section{Chapter 3 - Hydration of the Crust and Upper Mantle of the Hikurangi Plateau as it Subducts at the Southern Hikurangi Margin}

Chapter 3 presents the results from modelling controlled-source, onshore-offshore seismic data across the southern Hikurangi margin. The main objective of this chapter is to constrain the crustal and upper mantle P-wave velocity structure of the Hikurangi Plateau.

The main research questions to be answered in this chapter are:

1. What is the crustal thickness of the Hikurangi Plateau? Is it consistent with estimates from other studies nearby?

2. Is there evidence for anomalously fast upper mantle P-wave-speeds offshore Hikurangi margin?

3. Does the bending-induced normal faulting of the subducting plate near the Hikurangi trough result in the reduction of P-wave-speeds in the crust and the upper mantle? What are the implications for crustal and upper mantle hydration?

The results from this chapter provide insights into the crustal and upper mantle P-wave velocity structure and implications for crustal and upper mantle hydration of the subducting Hikurangi Plateau at the Hikurangi trough. This chapter has been published in Earth and Planetary Science Letters [Herath et al., 2020], with the title "Hydration of the crust and upper mantle of the Hikurangi Plateau as it subducts at the southern Hikurangi margin". I (Pasan Herath) was responsible for performing the data 
analysis, modelling and writing of the manuscript. Stuart Henrys, Tim Stern, and Martha Savage led the SAHKE project and collected the data. All these authors, Dan Bassett and Carolyn Boulton contributed to the interpretation of results and writing the manuscript.

Supplementary information for this chapter is given in Appendix A.

Chapter 4 - Evidence for a Lithosphere-Asthenosphere Boundary Zone of the Pacific Plate at the Southern Hikurangi Margin

The main objective of Chapter 4 is to investigate further the origin of a series of enigmatic late-arriving, far-offset wide-angle reflections observed in the onshore-offshore seismic data in Chapter 3. Initial investigations in Chapter 3 suggest that they may be reflections from the lithosphere-asthenosphere boundary of the Pacific Plate.

The main research questions to be answered in this chapter are:

1. Do these wide-angle reflections come from the lithosphere-asthenosphere boundary channel?

2. If yes, what do these wide-angle reflections reveal about the lithosphere-asthenosphere boundary?

3. What are the alternative explanations for the wide-angle reflections? Are they plausible?

The results from this study provide insights into the fine-scale structure at the lithosphere-asthenosphere boundary (LAB) and evidence for a more structured LAB which we refer to as the "LAB zone" here.

Supplementary information for this chapter is given in Appendix B.

Chapter $5-\mathrm{V}_{\mathrm{P}} / \mathrm{V}_{\mathrm{S}}$ Ratio, Material and Slip-rate Deficit Variations along Hikurangi Margin Forearc

Chapter 5 focuses on the identification of mode-converted seismic waves from airgun shots captured by an array of ocean bottom seismographs along the Hikurangi margin to estimate the compressional- to shear-wave velocity ratio $\left(V_{\mathrm{P}} / \mathrm{V}_{\mathrm{S}}\right)$ of the sediments and rocks above the subducting slab. 
With this, we expect to answer the following questions.

1. How does the $V_{P} / V_{S}$ ratio of the sediments and rocks above the subducting crust vary along the Hikurangi margin forearc? Does it show any relationship with the crustal composition and the subduction thrust slip behaviour?

2. How does the anisotropy of the sediments and rocks above the subducting crust vary along the forearc? Are the $\mathrm{V}_{\mathrm{P}} / \mathrm{V}_{\mathrm{S}}$ ratios affected by anisotropy?

It provides insights into the relationships between $V_{P} / V_{S}$ ratio, thickness and composition of the overriding crust, and the slip-rate deficits along the strike of the Hikurangi margin. It also provides insights into the seismic anisotropy of the Hikurangi margin forearc.

Supplementary information for this chapter is given in Appendix C.

\section{Chapter 6 - Thesis Summary}

Chapter 6 is provides a synthesis of the findings presented in the thesis and the answers to the research questions and the hypotheses tested in Chapters 3, 4 and 5. It also outlines their key findings, contributions, drawbacks and directions for future research.

The individual projects presented in Chapters 3, 4 and 5 have been undertaken by myself (Pasan Herath) under the supervision of Prof. Tim Stern and Prof. Martha Savage from Victoria University of Wellington and Dr. Dan Bassett and Dr. Stuart Henrys from GNS Science. These chapters have been compiled in a form appropriate for publication in peer-reviewed journals with their own abstracts, introductions, methods, results, discussion and conclusions sections. Thus, they have been written in first-person plural ("we"), which is the common standard for publications with more than one author. In addition, some repetition between Chapter 1 and these three chapters can be expected.

\subsection{Previous Studies on the Lithospheric Structure at the Hikurangi Margin}

Lithospheric structure at the Hikurangi margin has been explored using both passive-source and active-source seismic methods both onshore [e.g. Eberhart-Phillips et al., 2020; Henrys et al., 2013; Savage et al., 2007; Stern et al., 2015] and offshore [e.g. 
Barker et al., 2009; Bell et al., 2010; Henrys et al., 2006; Mochizuki et al., 2019; Yarce et al., 2019]. Onshore studies have provided constraints on seismic velocity and interface structure of different components of the lithosphere, from the forearc wedge of the Australian Plate down to the base of the subducting Pacific Plate. Offshore studies have provided constraints from the seafloor down to the top of the upper mantle beneath the Moho.

The following sections provide an overview of the lithospheric structure at the Hikurangi margin determined from these studies.

\subsubsection{Hikurangi Margin Forearc}

The Hikurangi margin forearc takes up the region from the volcanic arc to the trough of the overriding Australian Plate. The forearc wedge is built against a Mesozoic basement backstop of Torlesse Terrane greywackes [Barnes et al., 2010; Mountjoy and Barnes, 2011]. It consists of three major components (1) an inner foundation of Late Cretaceous and Paleogene, pre-subduction rocks; (2) an outer wedge of late Cenozoic accreted trench-fill turbidites; and a deforming cover sequence of Miocene to Recent shelf and slope basin sediments [Barnes et al., 2010]. The accretionary wedge of the forearc is characterised by long $(\sim 100 \mathrm{~km})$ thrust ridges and slope basins and major thrust faults, many of which splay from the plate interface to the surface [Wallace et al., 2009, and references therein].

Frontal accretion is observed to dominate in the southern Hikurangi margin, reflecting the high sediment supply in the region. The accretionary wedge here is characterised by contraction indicated by folding and the presence of Torlesse Supergroup greywackes from the fossil Gondwana subduction margin [Bland et al., 2015; Mortimer, 2004]. In contrast, the central and northern segments of the margin are characterised by backarc extension, less sediment accretion due to lower sediment supply and subducting seamounts [Barker et al., 2009; Bell et al., 2010].

The width of the accretionary wedge of the Hikurangi margin varies along the margin (Figure 1.2), with a maximum of about $70 \mathrm{~km}$ in the central part of the margin. The accretionary wedge narrows towards the southwest from $\sim 41^{\circ} \mathrm{S}$ and towards the northeast of Hawke Bay (Figure 1.2). The forearc wedge at the coastline is about 12-14 $\mathrm{km}$ thick except at Hawke's Bay (Figure 1.2), based on the plate interface model of 
Williams et al. [2013]. From onshore-offshore seismic data, Bassett et al. [2014] constrain average forearc P-wave velocities of $5.0 \mathrm{~km} / \mathrm{s}$ for the southern Hikurangi margin and 3.5-4.5 km/s for the northern Hikurangi margin. This along-strike variation in P-wave velocities is further verified from modelling ocean bottom seismograph data [Bassett et al., 2018].

A layer of low-velocity sediments is reported to exist between the overriding Australian Plate crust and the subducting Hikurangi Plateau crust [e.g. Chadwick, 1997; Henrys et al., 2013; Tozer, 2013], and is defined as a layer of subducting sediments.

\subsubsection{Hikurangi Plateau}

The subducting Pacific Plate at the Hikurangi margin contains the Hikurangi Plateau, an oceanic large igneous province [Coffin and Eldholm, 1994]. The Hikurangi, Ontong-Java and Manihiki Plateaus are considered to have formed together as a single super-plateau, the Ontong Java-Manihiki-Hikurangi Plateau, based on their similar formation ages, chemical composition, velocity structure and submarine emplacement [Taylor, 2006]. It is thought to have originated in the Lower Jurassic-Early Cretaceous as part of an oceanic spreading centre overlying a hotspot plume head [Hoernle et al., 2010]. The three plateaus, Ontong-Java, Manihiki and Hikurangi rifted apart from each other in the Early Cretaceous and were brought to their present positions by tectonic plate motions [Davy et al., 2008; Hochmuth et al., 2015; Taylor, 2006].

Oceanic plateaus have undergone extensive melting and modification after formation at a mid-oceanic ridge [Coffin and Eldholm, 1994]. Usually, the melting is thought to be due to the interaction with a mantle plume . However, alternative phenomena including melting due to a collision structure or large scale Rayleigh-Taylor instability [Tanton and Hager, 2000] and decompression melting of buoyant mantle that was removed from a cratonic root by subduction [Mochizuki et al., 2019] are also proposed as mechanisms facilitating the melting. As a result of the melting, mantle peridotite becomes depleted and the thickness of the overlying oceanic crust increases. Tectonic and geophysical implications of this process are: the plateau becomes more buoyant, because of both thickened crust and depleted mantle; and the P-wave-speeds increase in the depleted mantle [Jordan, 1978]. 


\subsubsection{Crustal Structure}

Gravity modelling estimates the crustal thickness of the Hikurangi Plateau to be $\sim 10$ $\mathrm{km}$ in the northern Hikurangi margin and $\sim 15 \mathrm{~km}$ in the southern Hikurangi margin [Davy and Wood, 1994]. Recent, controlled-source seismic data reveal a $10 \mathrm{~km}$ thick Hikurangi Plateau crust under the southwestern North Island [Henrys et al., 2013; Tozer et al., 2017] and $\sim 10 \mathrm{~km}$ thick crust overlain by a $\sim 1-2 \mathrm{~km}$ thick volcanoclastic layer offshore of the east coast of southern North Island [Mochizuki et al., 2019]. These crustal thickness estimates are supported by receiver function studies [Savage et al., 2007]. In the Hawkes Bay region, Bannister [1988] estimates a crustal thickness of 11-12 km, based on relocated earthquake hypocentres. Reyners et al. [2011], based on the distribution of seismicity suggests that the crust of the Hikurangi Plateau is $\sim 35 \mathrm{~km}$ thick. Thus the higher thickness of the oceanic crust of the Hikurangi Plateau compared to the regular oceanic crust $(\sim 7 \mathrm{~km}$ thick) is significant.

Controlled-source seismic data reveal P-wave velocities with varying gradients with a minimum of $\sim 5 \mathrm{~km} / \mathrm{s}$ at the top and a maximum of $\sim 7.5 \mathrm{~km} / \mathrm{s}$ at the bottom of the subducting Hikurangi Plateau crust in the southern Hikurangi margin [Henrys et al., 2013; Mochizuki et al., 2019; Tozer et al., 2017]. These estimates on P-wave velocities are supported by passive-source tomographic studies [Eberhart-Phillips et al., 2010, 2020].

\subsubsection{Lithospheric Mantle Structure}

Passive-source seismic studies on the eastern North Island along northeast-southwest oriented raypaths reveal high $\mathrm{P}$-wave-speeds of $8.50-9.00 \mathrm{~km} / \mathrm{s}$ within the mantle lithosphere of the Hikurangi Plateau [e.g. Bannister, 1988; Galea, 1992; Haines, 1979; Kayal and Smith, 1984]. A controlled-source, wide-angle reflection and refraction experiment in the eastern North Island, also oriented northeast-southwest, confirms the presence of a fast upper mantle layer with P-wave-speeds of $8.70-8.80 \mathrm{~km} / \mathrm{s}$, and indicates that it is not directly below the Moho, but underlies a regular mantle layer of thickness $\sim 8-10 \mathrm{~km}$ and P-wave-speeds of $8.10-8.20 \mathrm{~km} / \mathrm{s}$ [Chadwick, 1997]. All these studies measure upper mantle P-wave-speeds in the margin-parallel direction. One explanation for the high upper-mantle P-wave-speeds is that they are a result of anisotropy created by flow-induced orientation of mantle olivine crystals [Brisbourne and Stuart, 1998; Galea, 1992; Robinson, 1986]. Shear-wave splitting of SKS phases have also yielded margin-parallel fast directions in this region [Marson-Pidgeon and Savage, 
2004], but SKS phases cannot distinguish well what depths correspond to the anisotropy. A few passive-source seismic studies [Chong, 1982; Eberhart-Phillips et al., 2010; Robinson, 1986] and a controlled-source seismic study [Mochizuki et al., 2019] conducted along margin-perpendicular directions have also found the existence of fast upper mantle P-wave-speeds. Stern et al. [2020] also found the existence of fast upper mantle P-wave-speeds of up to $8.7 \mathrm{~km} / \mathrm{s}$. Reyners [2012] suggests that the lower crust of the proposed $\sim 35 \mathrm{~km}$ thick Hikurangi Plateau crust (Section 1.3.2.1) is transformed to eclogite to explain the high P-wave-speeds $(>8.5 \mathrm{~km} / \mathrm{s})$. However, estimates from recent studies on the Hikurangi Plateau (Section 1.3.2.1) show the crustal thickness is about 10-12 km. Another recently proposed explanation for the unusually high upper mantle P-wave-speeds is radial anisotropy created by a fabric specific to the gravitational collapse of a plume head [Stern et al., 2020]. This argument is supported by a similarly high upper mantle P-wave-speeds found in parts of the now dispersed Ontong-Java Plateau. Regardless of interpretation, the upper mantle of the Hikurangi Plateau has unusually high P-wave-speeds, and these wave-speeds are azimuthally isotropic.

Under the southern North Island, the thickness of the Pacific Plate is estimated to be $\sim 73$ $\mathrm{km}$ [Stern et al., 2015], suggesting a thickness of 60-63 km for the mantle lithosphere. A $\sim 10 \mathrm{~km}$ thick, low-velocity, sheared, melt-rich channel is proposed to exist at the base of the Pacific Plate under the North Island, and is interpreted as the lithosphereasthenosphere boundary channel of the subducting Pacific Plate [Stern et al., 2015]. 



\section{Chapter 2}

\section{Theory and Methods}

\subsection{Summary}

Chapter 2 outlines the theoretical and methodological framework relevant to the thesis. The chapter begins from an introduction to seismic waves and rays. A description on controlled-source seismology with an overview of controlled-source seismic surveys and survey geometries utilised in this thesis is given. Finally, a summary on raytracing-based travel-time modelling, seismic anisotropy, shear-wave splitting and synthetic seismogram generation using seismic wave propagation simulations adopted in the thesis is given.

\subsection{Seismic Waves and Rays}

Seismic waves are parcels of elastic strain energy that propagate outwards from a seismic source such as an earthquake or an explosion [Kearey et al., 2002] (Figure 2.1). There are two broad categories of seismic waves, namely body waves and surface waves. Body waves travel through the interior of the Earth. Surface waves propagate along the surface of the Earth.

In controlled-source seismology, the main focus is the use of body waves. There are two types of body waves, compressional or primary (P-) and shear or secondary (S) waves. Propagation velocities of body waves depend on the elastic moduli of the material, with P-waves being faster than S-waves. In P-waves, the direction of particle motion is along the direction of wave propagation. In S-waves, the direction of particle motion is perpendicular to the direction of propagation. There are two types of S-waves: $\mathrm{S}_{\mathrm{V}}$ which is polarised in the vertical plane and $\mathrm{S}_{\mathrm{H}}$ which is polarised in the horizontal 
plane. P- and $\mathrm{S}_{\mathrm{V}}$-waves are coupled with each other when they interact with horizontal boundaries, whereas $\mathrm{S}_{\mathrm{H}}$ waves remain separate [Stein and Wysession, 2003].

In a homogeneous and isotropic medium, seismic body waves generated by a point source travel at the same velocity (as defined by the elastic properties of the medium) in all directions. At a given time, the propagation of the seismic waves can be approximated by a spherical wavefront, defined as the locus of all points which the pulse has reached at a particular time. Seismic rays are defined as thin pencils of seismic energy travelling along ray paths that, in isotropic media, are everywhere perpendicular to wavefronts [Kearey et al., 2002] (Figure 2.1).

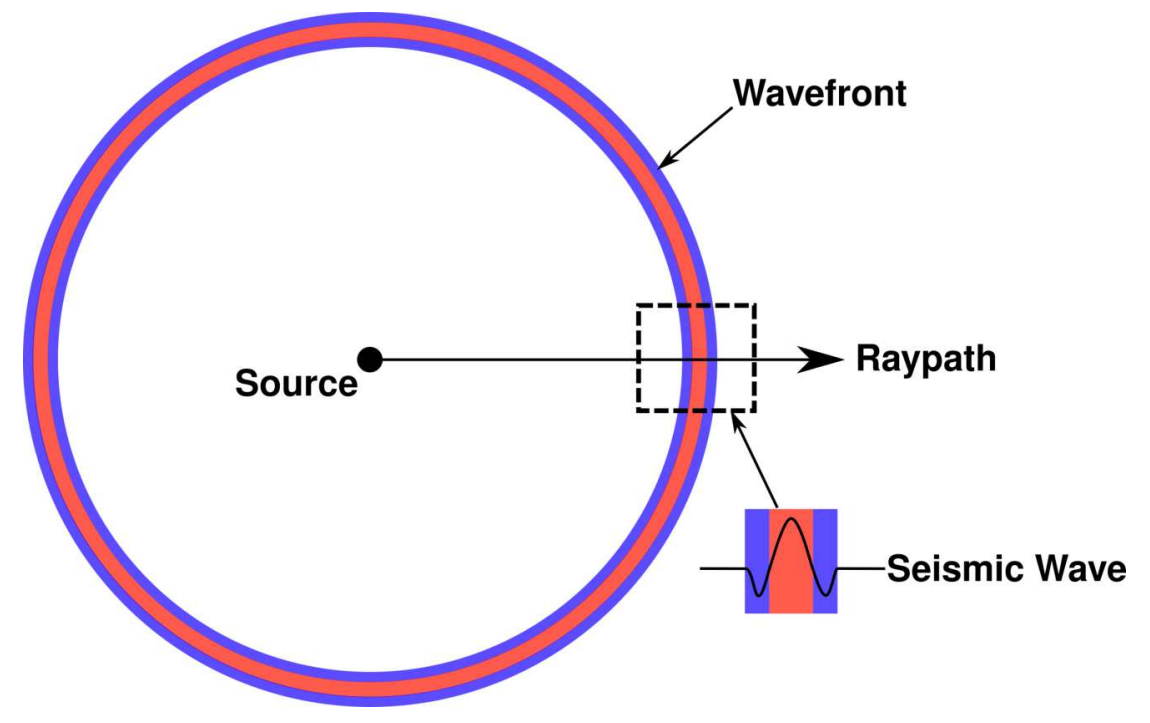

Figure 2.1 A wavefront generated from a point source. Raypaths are perpendicular to wavefronts at any instance in isotropic media.

Upon reaching an interface with another medium with different seismic velocities, the wavefronts reflect and refract and also undergo mode conversion ( $\mathrm{P}$ to $\mathrm{S}$ and vice versa). This can be represented by raypaths (Figure 2.2). Refraction and reflection angles obey Snell's Law, which states that along a ray, the ray parameter $(p)$ remains constant, where $p=\sin (i) / v$. Here, $i$ is the inclination of the ray, and $v$ is the velocity.

The displacement coefficients as a function of incidence angles for a plane wave incident on an interface can be calculated using Zoeprittz equations [Aki and Richards, 2002].

Seismographs record the ground displacement, velocity or acceleration induced by the incoming reflected and refracted wavefronts in the form of a seismogram. Therefore, a seismogram is a convolution of the source-time function, travel-path and receiver effects. 


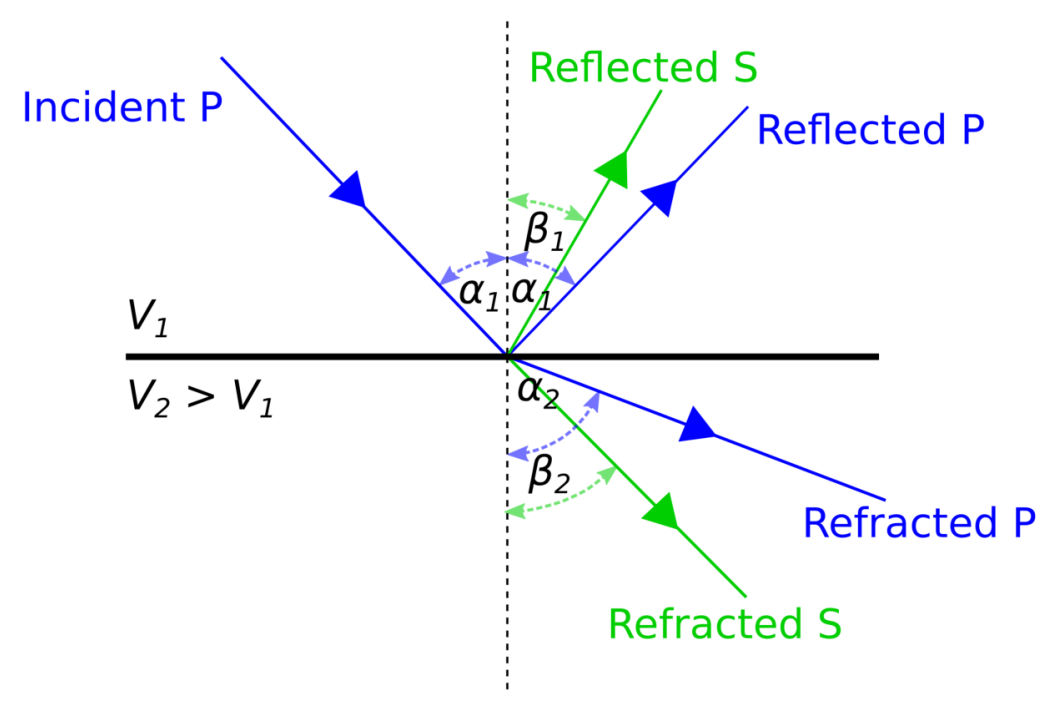

Figure 2.2 Schematic diagram showing the partitioning of an obliquely incident P-wave at an interface.

\subsection{Controlled-source Seismology}

Controlled-source seismology is based on the use of artificial sources to generate seismic waves and recording them by an array of seismographs. As the origin location and time of the sources can be known precisely with the use of GPS clocks, the uncertainties in model parameter determination from modelling controlled-source seismic data are lower when compared with those from modelling passive-source seismic data. The capability to have a densely spaced sources and/or receivers and repeat the same source signature are also advantages of controlled-source seismology. However, these advantages come at a cost, in terms of the funding required and difficulties in handling the logistics for regional scale experiments.

Artificial sources used in controlled-source seismology include explosives detonated in boreholes, ground impact from vibrations and hammers mounted on trucks and marine airgun arrays. The choice of a particular source depends on where the seismic survey is carried out and also other aspects like the required depth of penetration and the degree of resolution of the subsurface structures imaged. The widely used controlled-sources for experiments that focus on imaging the deep interior of the Earth ( $>10 \mathrm{~km}$ deep) are explosive blasts (e.g. dynamite) [e.g. Bean and Jacob, 1990; Stern et al., 2015] and airgun shots [e.g. Henrys et al., 2013; Van Avendonk et al., 2011]. The depth of penetration and the resolution of the imaged subsurface structures can be increased by improving the source parameters, i.e. the weight of explosives used in the case of dynamite or the 
airgun volume and charging pressure in the case of airgun shots. Increasing the source parameters results in higher source energy and a wide spectrum of frequencies.

The recorders which record the seismic energy are known as seismographs. Seismographs typically consist of geophones that record the ground motion velocity by measuring the voltage generated by a metallic mass suspended inside a magnetic field. Most of the controlled-source experiments on land focus on using P-waves to model the subsurface structures and velocities. These experiments tend to use seismographs with a vertical geophone to record the incoming P-waves. In experiments which focus on recording both P- and S-waves, two more horizontal geophones that are orthogonal to each other are included in the seismograph to capture ground motion from S-waves.

In a controlled-source experiment, an array of seismographs is stationed at increasing offsets from the source (Figure 2.3). The array of seismographs record the reflected and refracted waves from the controlled-source in the subsurface which are used to derive the velocity structure underneath.

Multichannel seismic reflection surveys and wide-angle reflection and refraction surveys are two categories of controlled-source seismic surveys. The main differences between the two categories are in the survey geometry and the processing of the data. Although they differ from each other, multichannel seismic reflection surveys complement wide-angle surveys by providing constraints on the near surface velocity structure.

\subsubsection{Multichannel Seismic Reflection Surveying}

Multichannel seismic (MCS) surveys are conducted both onshore and offshore. Most modern onshore MCS surveys use explosives, Vibroseis or hammer impacts as the seismic source recorded by long arrays of geophones. In offshore MCS surveys, airgun shots from a seismic vessel are recorded by a streamer of hydrophones towed behind the airgun array in the same seismic vessel. These surveys provide useful information on the variations of the subsurface structures, and indirect information on the seismic-wave velocities (i.e. stacking velocities). This is because most of the refracted arrivals which directly resolves the velocity structure are muted at the processing stage [White, 2012]. Structures and seismic wave-speeds in the deeper regions of the 


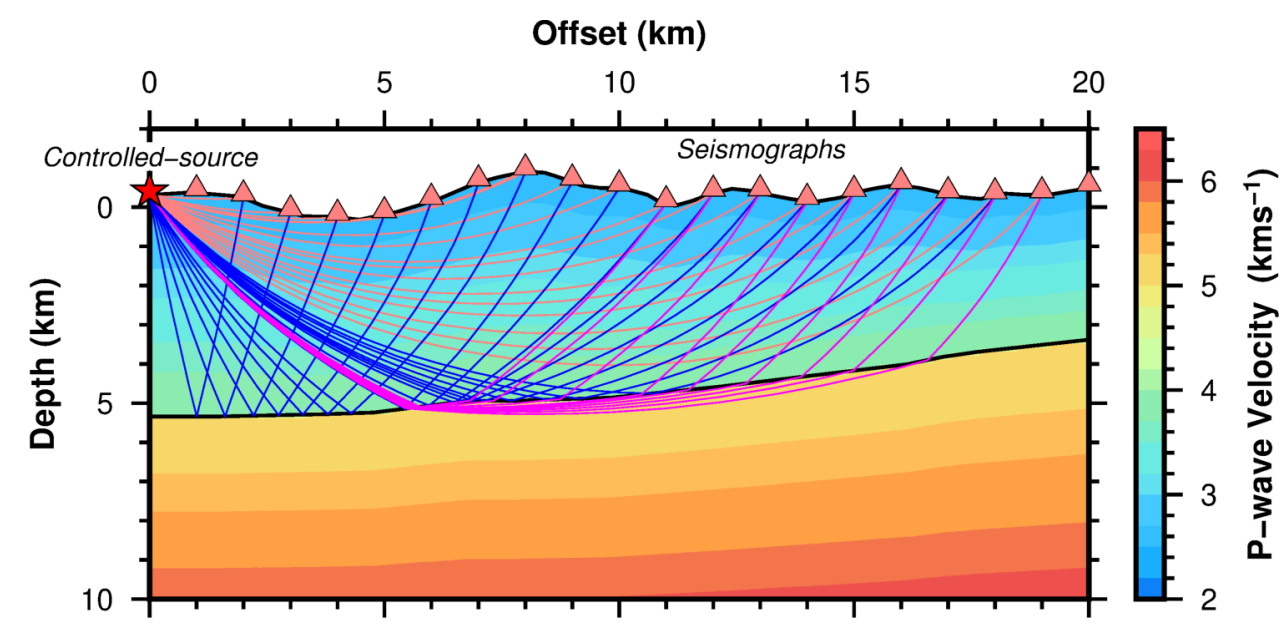

Figure 2.3 Layout of a typical, on-land wide-angle seismic survey. Coloured raypaths indicate the refracting waves in the upper layer (light red), in the lower layer (magenta) and the reflected wave from the interface in between the two layers (blue).

crust and upper mantle may not be resolved due to the smaller source-receiver offsets $(\leq 12 \mathrm{~km})$ used in these surveys [Kearey et al., 2002].

\subsubsection{Wide-angle Reflection and Refraction Seismic Surveying}

Wide-angle seismic surveys aimed at exploring structures and velocities in regions of the crust and upper mantle use larger source-receiver offsets $(>50-100 \mathrm{~km})$ and hence can sample deeper regions of the Earth (up to 20-30 km) or even deeper with much larger source-receiver offsets. The seismic sources are either explosives or airguns depending on where the target of interest is located. Since wide-angle seismic surveys are used in crustal-scale experiments, the seismic sources are designed to contain low frequencies because due to the longer travel paths, higher frequencies are absorbed by the rocks [White, 2012]. In onshore surveys, both the sources and seismographs are located on-land. In the case of offshore surveys, ocean bottom seismographs (OBS) are widely used, and the sources are usually airgun shots from a seismic vessel. A specialist type of wide-angle seismic survey that integrate offshore airgun shots from a seismic vessel and onshore seismographs is known as the onshore-offshore seismic profiling technique [Okaya et al., 2003].

Airgun shots used in ocean-bottom and onshore-offshore seismic surveys are recorded both in hydrophones of the streamer and in the ocean-bottom/onshore seismographs, which provide both multichannel reflection and wide-angle reflection and refraction data. However, in crustal-scale experiments, the seismic lines are acquired twice using a smaller shot spacing $(<50 \mathrm{~m})$ for multichannel seismic data and a relatively longer 
shot spacing (100-200 m) for wide-angle reflection and refraction data. Nevertheless, this allows us to use these two different active-source seismic data, multichannel and wide-angle, to obtain a well constrained image of the shallow and deep subsurface.

Wide-angle reflection and refraction seismic surveys are mostly conducted as two-dimensional surveys, as it is simple to conduct, less expensive and easy to interpret.

\subsubsection{Onshore-offshore Seismic Surveys}

The onshore-offshore seismic profiling technique is particularly useful when the target of interest spans through the transition from ocean to land and parallel to the coastline (e.g. plate boundary fault in a subduction zone) (Figure 2.4a). Source-receiver offsets can be progressively increased by recording airgun shots of a seismic vessel moving away from the coastline at the seismographs on land. Thus deeper velocity structure and interfaces can be resolved. The closely spaced airgun sources provide high resolution in the common receiver gathers that are generated from the continuously recorded data in the onshore seismographs. This high resolution common receiver gathers facilitate quick and easy identification of seismic phases.

In a single-sided onshore-offshore seismic survey, a refraction velocity versus dip ambiguity normally arises with unidirectional shooting [Okaya et al., 2003]. However, when many land instruments are deployed in an onshore-offshore transect, the large number of common receiver gathers compensates for the unreversed shooting, particularly when analysed using modern tomography and ray trace methods [Okaya et al., 2003]. Moreover, if there are enough onshore receivers, shot-gathers can be made, which are an effective reversal of the receiver-gathers.

The commonly observed seismic phases in a common receiver gather in a typical onshore-offshore seismic survey at a subduction zone setting are: refraction in the overriding continental crust $\left(\mathrm{P}_{\mathrm{g}}\right)$; wide-angle reflection from the top of the subducting oceanic crust $\left(\mathrm{P}_{\mathrm{c}} \mathrm{P}\right)$; refraction in the oceanic crust $\left(\mathrm{P}_{\mathrm{o}}\right)$; wide-angle reflection from the Moho $\left(\mathrm{P}_{\mathrm{m}} \mathrm{P}\right)$ and the upper mantle refraction $\left(\mathrm{P}_{\mathrm{n}}\right)$ (Figure 2.4$)$. 
(a)

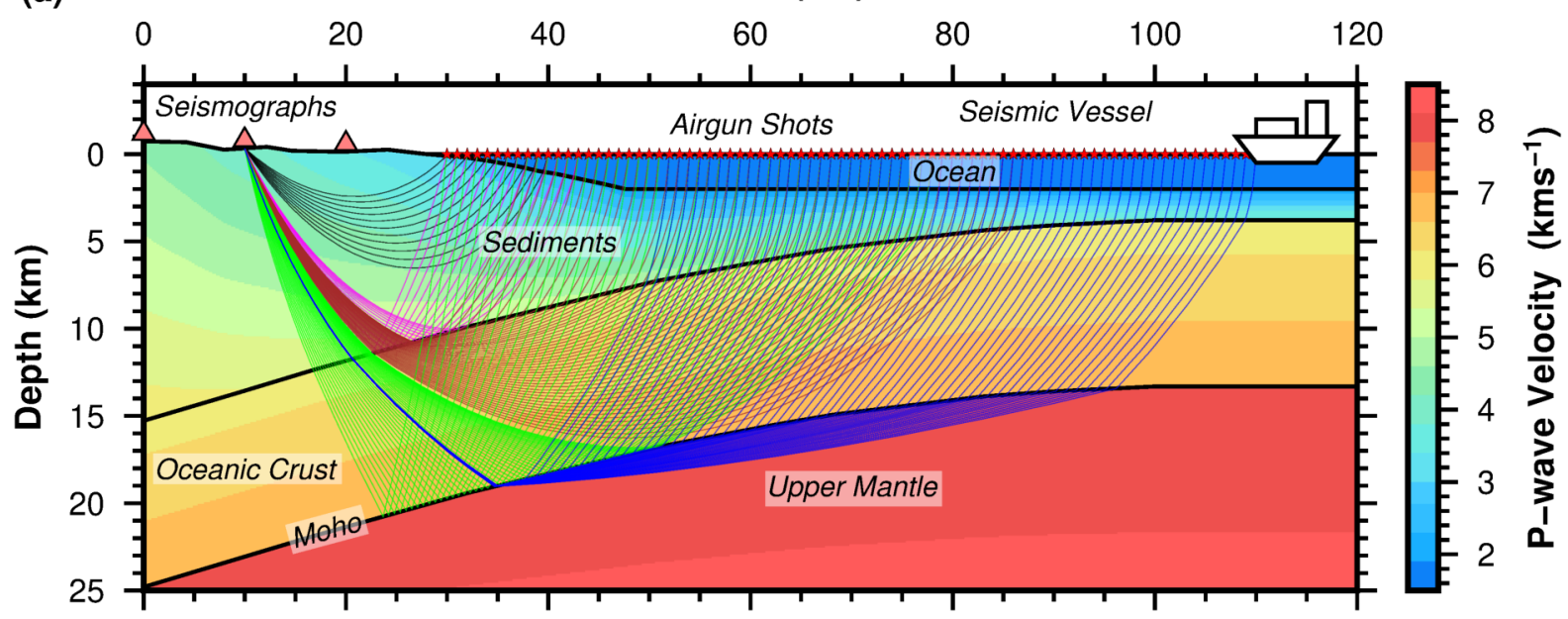

(b)

Offset (km)

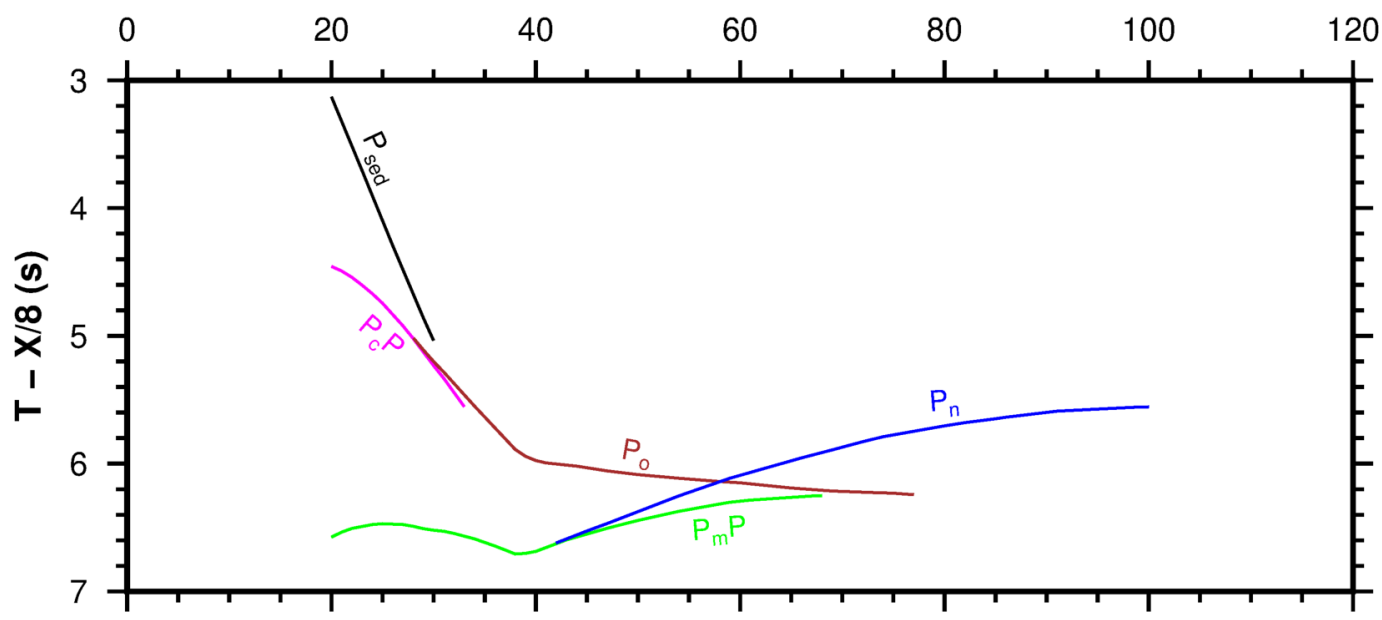

Figure 2.4 (a) Layout of an onshore-offshore seismic survey at a subduction zone setting. Raypaths indicate the key seismic reflections and refractions. (b) Travel-times for the reflected and refracted waves colour-coded as in (a).

\subsubsection{Ocean Bottom Seismic Surveys}

Ocean bottom seismic surveys are conducted when the target of interest lies beneath the ocean floor. Here, ocean bottom seismographs (OBS) are deployed on the ocean floor at a spacing of a few kilometres. These OBSs record the direct, reflected, refracted and waves off airgun shots from a seismic vessel (Figure 2.5a).

The commonly observed seismic phases in a vertical component common receiver gather in a typical ocean bottom seismic survey at an oceanic lithosphere setting are: direct water wave $\left(P_{w}\right)$; refraction in the sediments overlying the oceanic crust $\left(P_{\text {sed }}\right)$; wide-angle reflection from the top of the oceanic crust $\left(\mathrm{P}_{\mathrm{c}} \mathrm{P}\right)$; refraction in the oceanic crust $\left(\mathrm{P}_{\mathrm{o}}\right)$; wide-angle reflection from the Moho $\left(\mathrm{P}_{\mathrm{m}} \mathrm{P}\right)$ and the upper mantle refraction $\left(P_{n}\right)$ (Figure 2.5$)$. 
Airgun sources contain P-wave energy only. However, upon the oblique incidence at an interface with a significant impedance contrast (e.g. ocean bottom, sediment-basalt interface), mode-conversions could occur where P-waves convert to S-waves or vice versa. When ocean bottom seismic experiments target such mode-conversions to derive the shear-wave velocity structure or the seismic velocity ratio $\left(V_{P} / V_{S}\right)$ structure, the OBSs are equipped with two orthogonal horizontal geophones in addition to the vertical component geophone as the incoming S-wave energy to the OBSs are primarily recorded by the horizontal components. For example, in a typical ocean bottom seismic experiment, mode-conversions can occur at the ocean bottom (ob) for down-going waves from airgun shots $\left(\mathrm{PS}_{\mathrm{ob}} \mathrm{S}_{\mathrm{x}} \mathrm{x}=[\mathrm{sed}, \mathrm{o}, \mathrm{n}]\right.$ phases in Figure 2.5). Mode-conversions can also occur at the Moho $(\mathrm{m})$ and the top of oceanic crust (c) for up-going waves from refractions $\left(\mathrm{PP}_{\mathrm{x}} \mathrm{S}_{\mathrm{y}}\right.$ where $\mathrm{x}=[\mathrm{o}, \mathrm{n}]$ and $\mathrm{y}=[\mathrm{m}, \mathrm{c}]$ in Figure 2.5).

\subsection{Processing Wide-angle Seismic Data}

Seismographs record the ground motion continuously. The continuously recorded data at each seismograph are sorted into a common receiver gather to visualise the refracted, reflected and mode-converted arrivals as a function of source-receiver offset. The common receiver gathers are processed further to improve the signal-to-noise ratio to facilitate the identification of different seismic phases.

\subsubsection{Common Receiver Gathers}

Wide-angle, controlled-source seismic data acquired in onshore-offshore and ocean bottom seismic surveys are usually visualised using common receiver gathers. Common receiver gathers are produced by extracting seismograms from the continuously recorded data at each receiver starting at the onset of each airgun shot. The extracted seismograms are plotted against increasing and/or decreasing offsets between the source and receiver.

Because of the reciprocal property of a seismic wave as it travels between a shot and a receiver, a receiver gather for any given seismograph can be viewed and modelled as a shot at the seismograph being recorded at every airgun shot point.

When the objective of the study is modelling the P-wave velocity structure, common 
(a)

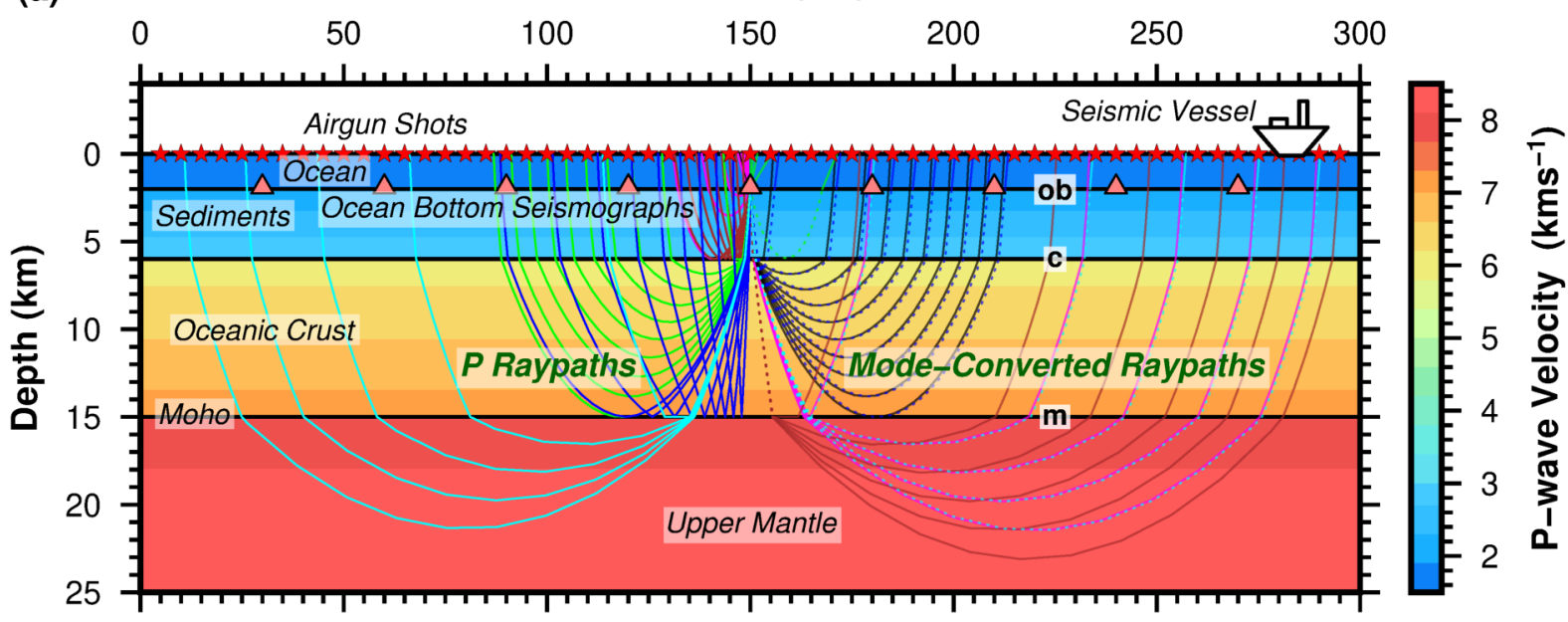

(b)

Offset (km)

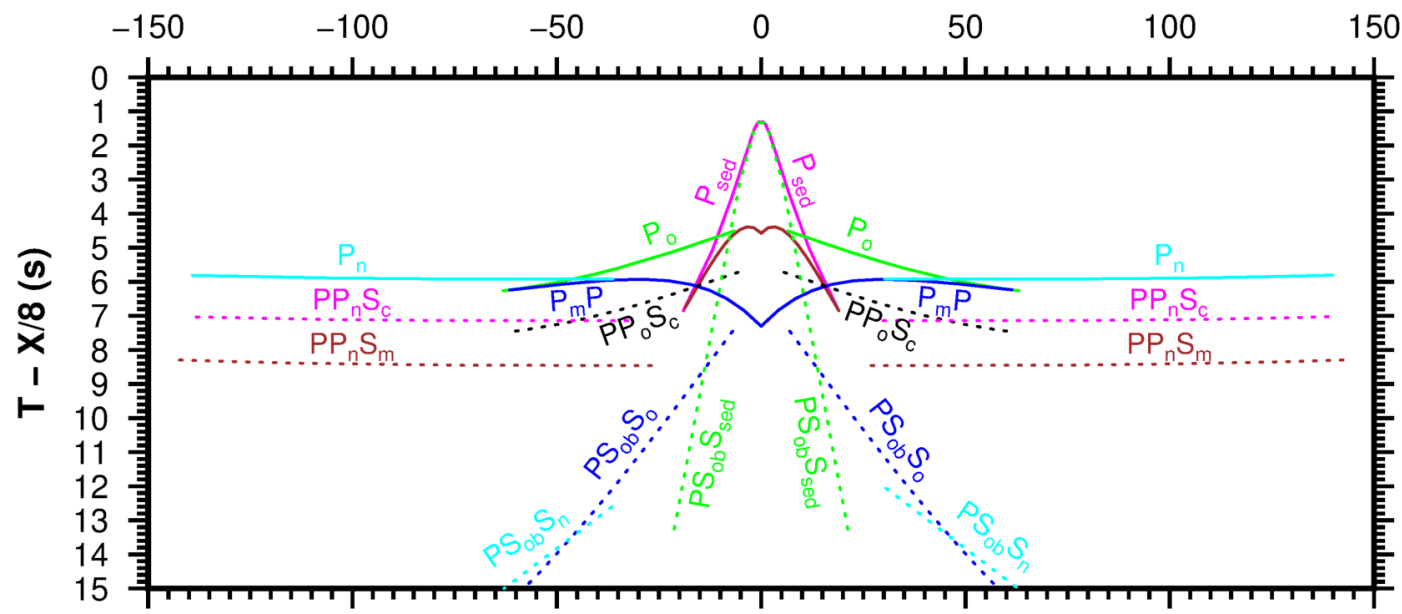

Figure 2.5 (a) Layout of an ocean bottom seismic survey in an oceanic setting. Raypaths on the left half indicate the common P-wave refractions and wide-angle reflections. Raypaths on the right half indicate the possible P- to S- mode conversions at the seafloor for downgoing waves and at the sediment-oceanic crust interface and Moho for upgoing waves. S- raypaths are indicated by dotted lines (b) Travel-times for the reflected and refracted P-waves (solid lines) and for the P- to S-converted waves (dotted lines) in (a). Travel-times are colour-coded as in (a).

receiver gathers are produced using the continuously recorded data in the vertical $(\mathrm{Z})$ component of the seismograph. However, when modelling the S-wave velocity structure, common receiver gathers of the two horizontal components are also produced as S-waves are easily identifiable in them.

The typical length of the time axis of a common receiver gather is $30-60$ seconds as these experiments are aimed at resolving the deeper velocity structure from refractions and wide-angle reflections. However, such long travel-time axes usually create visualisation issues. This is overcome by reducing the travel-time axis by the application of a reduction velocity (Figure 2.4b, 2.5b) where the travel-time corresponding to the offset with the reduction velocity is reduced from the true 
travel-time (Equation 2.1). Applying a reduction velocity also has advantages in identifying different seismic phases.

$$
\text { Reduced Travel-time }=\text { True Travel-time }-\frac{\text { Offset }}{\text { Reduction Velocity }}
$$

\subsubsection{Improving Signal-to-noise Ratio}

Waveforms in the common receiver gathers contain both signal and noise. Signal is the part of the waveform which contains information related to the subsurface geology and structures being explored. Noise could be categorised into two types, random and coherent. Random noise is the statistically random noise usually due to environmental factors (e.g. ground vibration due to traffic, electric wires, wind, flowing water, rain). Coherent noise on the other hand is generated by the experiment itself (e.g. surface waves), but is of no interest in most studies [Kearey et al., 2002].

The raw common receiver gathers are processed further before the identification of refractions and wide-angle reflections. The processing includes the application of different operations on the waveforms that helps increase the signal-to-noise ratio and coherency of arrivals. In this thesis, processing of the common receiver gathers are carried out using GLOBE ${ }^{T M}$ Claritas suite of seismic processing software.

\subsubsection{Frequency Filtering}

Frequency filtering is applied when the signal and noise in the waveforms have different frequency content. The signal in the waveform can be separated on this basis. Different types of frequency filters can be applied to retain the required frequencies in the waveform. Thus waveforms can be low-pass, high-pass, band-pass and band-reject filtered depending on the requirement. Frequency range of the filter is usually defined by four corner frequencies, with a broader taper $(>1 \mathrm{~Hz})$ on either sides to avoid artefacts in the filtered output.

Common receiver gathers used in this thesis are bandpass filtered using combinations of corner frequencies in the range 1-20 Hz using a Butterworth bandpass filter. BUTTERFILT processor in GLOBE ${ }^{T M}$ Claritas is used for this. 


\subsubsection{Automatic Gain Control}

Automatic gain control (AGC) is an amplitude scaling operation which can improve the visibility of weak and/or late-arriving events in which the amplitudes have decayed due to attenuation or spherical divergence. The mean or the median absolute amplitude of a user-defined time window centred at each sample in the trace is calculated as the scaling factor. The samples are multiplied by this scaling factor.

AGC processor in GLOBE ${ }^{T M}$ Claritas is employed with time-windows ranging from 5,000 to $20,000 \mathrm{msec}$ in processing the common receiver gathers.

\subsubsection{Runmixing}

Runmixing replaces the central trace with a stacked trace generated by summing up the amplitudes of an odd number of adjacent traces. Amplitudes in each trace are multiplied by weights, usually decreasing from the central trace, before summation. Thus the application of a runmixing operation on the waveforms results in the constructive interference of coherent signal and destructive interference of random noise. This is particularly useful in boosting up the amplitudes of weak, coherent arrivals across traces.

In the thesis, the RUNMIX processor in GLOBE ${ }^{T M}$ Claritas is used with 5 traces. The traces are weighted by $[0.1,0.2,0.4,0.2,0.1]$ to maintain a sum of 1.0 to preserve relative amplitudes.

\subsubsection{Deconvolution}

Deconvolution is the process that leads to compressing the basic wavelet (source wavelet) in the seismogram, attenuating reverberations (ringiness) and short period multiples. Therefore, it increases the temporal resolution and provides a more realistic representation of the impulse response of the subsurface [Yilmaz, 2001].

Predictive deconvolution was applied using the DECONW processor in GLOBE ${ }^{T M}$ Claritas, using variable filter and gap lengths in this thesis.

\subsection{Phase Identification}

Refracted phases in common receiver gathers are easily distinguishable with their linear moveout. However, perturbations to this linear trend could be introduced by 
the effects of anisotropy, velocity gradients and lateral variations in structure [White, 2012]. When the refracting interface is horizontal, the reciprocal of the gradient of the refraction yields the the P-wave velocitiy of the refraction. However, when the interface is dipping, the gradient of the refracted phase in the common receiver gather provides an apparent velocity of the refraction, which is higher than the actual velocity when shooting up-dip and lower than the actual velocity when shooting down-dip [Kearey et al., 2002].

Linear move-out (LMO) operators apply a reduction velocity to the travel-time axes of the common receiver gathers, which flattens the refracted phases that have a velocity similar to the reduction velocity. Therefore LMOs are used to estimate the apparent velocities of refracted phases. This is particularly useful in the identification of refractions from the crust and upper mantle, which commonly have different velocities of $\sim 6 \mathrm{~km} / \mathrm{s}$ and $\sim 8 \mathrm{~km} / \mathrm{s}$, respectively.

Wide-angle reflections appear as parabolas in common receiver gathers and are identified based on their asymptotic relationship to a refracted phase with increasing source-receiver offsets. For example, in Figure 2.5, $\mathrm{P}_{\mathrm{m}} \mathrm{P}$ is observed to converge with $\mathrm{P}_{\mathrm{o}}$ when the source-receiver offsets increase. Thus, the wide-angle reflection from the base of a particular layer has an asymptotic relationship with the refraction in the same layer.

Furthermore, when enigmatic phases are observed in a common receiver gather, synthetic travel-time calculations using a simple and representative velocity model can be used to understand their origins.

Travel-times of the identified refractions and wide-angle reflections are picked. A pick uncertainty for each picked travel-time is assigned based on the dominant frequency of the arrival, source-receiver offset and signal-to-noise ratio.

\subsection{Travel-time Modelling}

Travel-time modelling is a widely used technique with controlled-source seismic data. Travel-time modelling based on the geometric ray theory calculates the travel-time between a seismic source and a receiver. Calculation of the travel-time assumes Fermat's principle which states that, of the many possible paths between two points, 
the seismic ray follows the path that gives the shortest travel-time between the points [Lowrie, 2007]. Travel-times of the seismic phases are calculated by integrating the travel-time for each segment of the raypaths predicted by the geometric ray theory. Although the geometric ray theory does not fully describe important aspects of wave propagation, it is widely used because it often greatly simplifies the analysis and gives the correct answer or a good approximation [Stein and Wysession, 2003]. It is also quick and computationally cheap.

The primary objective of travel-time modelling is to develop a seismic velocity model that can fit the observed travel-times of refractions and wide-angle reflections. This is achieved by comparing the travel-times of the observed refracted and wide-angle reflections with the travel-times calculated for the same seismic phases based on a velocity model.

\subsubsection{Raytrace Modelling}

Raytrace modelling or raytracing is a technique used in travel-time modelling which implements the principles of geometric ray theory. In this, a velocity model is modified iteratively until a good fit between the observed and calculated travel-times is obtained. A final velocity model can obtained by forward modelling or inverse modelling. In forward modelling, the user updates the velocity model iteratively until a good fit between the observed and calculated travel-times is obtained. This can be time consuming and it does not provide estimates of model parameter resolution, uncertainty and non-uniqueness. Inverse modelling based on a particular norm can minimise the time needed for modelling and also provide estimates of model parameter resolution, uncertainty and non-uniqueness. But this relies greatly on the presence of a good ray coverage between the sources and receivers. In modelling travel-times, raytrace modelling is done using computer codes. Rays between a source and a receiver are traced though a discretised velocity model.

In this thesis, travel-time modelling in a forward model raytracing approach is carried out using RAYINVR [Zelt and Smith, 1992]. RAYINVR model parametrisation is based on a layered, variable-block-size representation of 2D isotropic velocity structure [Zelt and Ellis, 1988; Zelt and Smith, 1992]. Layer boundaries in the model are defined by nodes and velocities within each layer are defined by velocities at the top and the bottom boundaries (Figure 2.6). A layer boundaries thus can have topography and 
velocity discontinuities across them. Within a layer, velocity gradients can be introduced both horizontally and vertically. This is an advantage of RAYINVR, as modelling regions of the Earth with complex structures and velocity variations often requires such parametrisation capabilities.

In RAYINVR, rays are traced through the velocity model using zero-order asymptotic ray theory which involves the solving of a pair of first-order ordinary differential equations (Equations 2.2 and 2.3) efficiently by a Runge-Kutta method. Snell's law is applied at layer boundaries. In Equations 2.2 and 2.3, $\theta$ is the angle between the tangent to the ray and the z-axis, $v$ is the wave velocity, and $v_{x}$ and $v_{z}$ are partial derivatives of the velocity with respect to $x$ and $z$ coordinates [Zelt and Smith, 1992].

$$
\begin{gathered}
\frac{d x}{d z}=\tan \theta \\
\frac{d \theta}{d z}=\frac{v_{z} \tan \theta-v_{x}}{v}
\end{gathered}
$$

The travel-times are computed by the discrete form of the travel-time equation (Equation 2.4).

$$
t=\sum_{i=1}^{n} \frac{l_{i}}{v_{i}}
$$

Here, $t$ is the travel-time. $l_{i}$ is the path length and $v_{i}$ is the velocity of the $i^{\text {th }}$ ray segment.

After the parametrisation of an initial velocity model based on available constraints (e.g. topographic and bathymetric data, multichannel seismic reflection data), modelling the velocity structure is carried out with a top-down approach, in which the shallower velocity and interface structure is modelled first using seismic phases that refract and reflect from shallower layers, and working down the model progressively using later phases. By doing so, ambiguities and errors in the shallower layers do not propagate into deeper layers, which otherwise would result in incorrect velocity and depth determinations in the deeper parts of the model. 


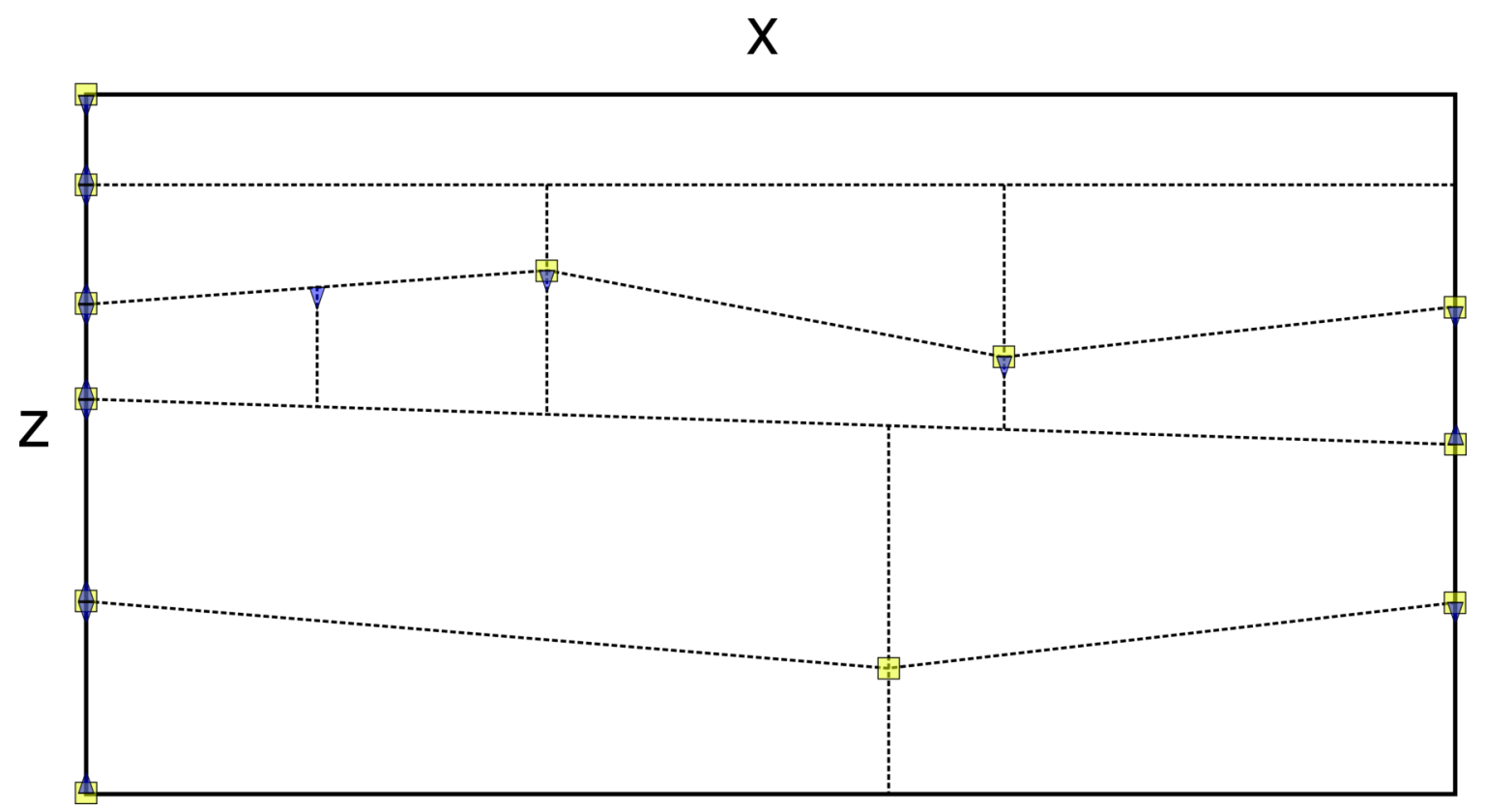

Figure 2.6 RAYINVR model parametrisation (modified after Zelt and Smith [1992]). Solid rectangle indicates the model domain. Black dashed lines are layer boundaries. Yellow squares are boundary nodes. Upright blue triangles are velocity nodes at the lower boundary and inverted blue triangles triangles are velocity nodes at the upper boundary for a particular layer.

\subsubsection{Uncertainties}

\subsubsection{Uncertainties with Data}

Systematic errors in modelling travel-times using raytracing methods may arise due to (a) equipment recording errors of phase arrival times, due to timing errors within the data recording system and/or (b) interpretation errors when picking phase arrivals [Tozer, 2013]. In the seismic data used in this thesis, equipment timing errors were not detected and if there had been, they had been corrected after the data collection. Errors in picking phase arrivals in the seismic data arise as a result of the width of the wiggle (dependant on frequency), ambiguities in picking the first break and cycle skips. Such interpretation errors are common. This type of error in incorporated into each traveltime pick as a pick error for each phase. It is mainly dependant on the frequency of the arrivals, source-receiver offset and the signal-to-noise ratio.

Goodness-of-fit of the observed and calculated travel-times in raytracing is measured by two statistical parameters, the root mean squared of travel-time misfits $\left(\mathrm{T}_{\mathrm{RMS}}\right)$ and $\chi^{2}$. $\mathrm{T}_{\text {RMS }}$ is a measure of how well the predicted travel-times match with the observations. $\chi^{2}$ on the other hand takes into account how well the predicted travel-times match with the observations relative to their pick uncertainty. In the presence of a large number of observed travel-time picks, normalised $\mathrm{T}_{\mathrm{RMS}}$ and $\chi^{2}$ are calculated based on Equations 
2.5 and 2.6. A model is considered to best fit the observations when the $\mathrm{T}_{\mathrm{RMS}}$ is minimal, possibly less than or equal to the pick errors, or when $\chi^{2}$ is equal to 1 . In the best-fit model, travel-time misfits are expected to have a normal distribution with zero mean.

$$
\begin{aligned}
& \text { Normalised } \mathrm{T}_{\mathrm{RMS}}=\sqrt{\frac{1}{n} \sum_{i=1}^{n}\left[t_{\mathrm{i}} c a l c-t_{\mathrm{i}} o b s\right]^{2}} \\
& \text { Normalised } \chi^{2}=\frac{1}{n-1} \sum_{i=1}^{n}\left[\frac{t_{\mathrm{i}} c a l c-t_{\mathrm{i}} o b s}{\sigma_{\mathrm{i}}}\right]^{2}
\end{aligned}
$$

In Equations 2.5 and 2.6, $n$ is the total number of rays traced, $t_{\mathrm{i}} c a l c$ is the calculated travel-time, $t_{\mathrm{i}}$ obs is the observed travel-time and $\sigma_{\mathrm{i}}$ is the pick uncertainty.

\subsubsection{Model Parameter Uncertainties}

Non-uniqueness or the presence of a number of solutions that can explain the observations is a key issue in geophysical modelling. For example, in travel-time modelling, the interface from which a wide-angle reflection occurs can be shallower or deeper depending on the velocities. This solution space can be inspected by using perturbation and grid-search tests for multiple model parameters (e.g. depth of a boundary node and the velocity) [e.g. Tozer et al., 2017]. This is particularly important when a forward model raytracing approach is implemented as it cannot provide estimates of model parameter uncertainties and/or non-uniqueness.

In addition, ray density plots are computed by calculating the number of rays travelling through a grid of cells in the model domain. Ray density plots provide insights on ray coverage and thus indicate which parts of the model are well constrained by raytrace modelling.

\subsection{Seismic Anisotropy}

In seismology, it is often assumed that the Earth is composed of isotropic material, in which the elastic properties do not change with direction. The stiffness tensor [Stein and Wysession, 2003] that defines the elastic constants of the material, (i.e. seismic wave velocities) can be explained by the Lame parameters $\lambda$ and $\mu$. In matrix form, the 
stiffness tensor for an isotropic material is given in Equation 2.7.

$$
C_{m n}=\left[\begin{array}{cccccc}
\lambda+2 \mu & \lambda & \lambda & 0 & 0 & 0 \\
\lambda & \lambda+2 \mu & \lambda & 0 & 0 & 0 \\
\lambda & \lambda & \lambda+2 \mu & 0 & 0 & 0 \\
0 & 0 & 0 & \mu & 0 & 0 \\
0 & 0 & 0 & 0 & \mu & 0 \\
0 & 0 & 0 & 0 & 0 & \mu
\end{array}\right]
$$

The isotropic P- and S-wave velocities, $V_{p}$ and $V_{s}$, respectively are related to the Lame parameters as in Equations 2.8 and 2.9, where $\rho$ is the density of the material.

$$
\begin{gathered}
V_{p}=\sqrt{\frac{\lambda+2 \mu}{\rho}} \\
V_{s}=\sqrt{\frac{\mu}{\rho}}
\end{gathered}
$$

However, there is increasing evidence that the assumption of isotropy in seismology in many parts of the Earth is an oversimplification. Therefore, we have to consider the effects of anisotropy, where seismic wave velocities change with direction. Anisotropy in the Earth can occur due to effects of deformation on cracks and minerals that are intrinsically anisotropic.

In the most general anisotropic case, to define the stiffness tensor of an anisotropic material, 21 independent elastic constants are required (Equation 2.10). The stiffness tensor (Equation 2.10) is symmetric where $C_{12}=C_{21}, C_{23}=C_{23} \ldots, C_{56}=C_{65}$, and thus has only 21 independent elastic constants. 


$$
C_{m n}=\left[\begin{array}{llllll}
C_{11} & C_{12} & C_{13} & C_{14} & C_{15} & C_{16} \\
C_{21} & C_{22} & C_{23} & C_{24} & C_{25} & C_{26} \\
C_{31} & C_{32} & C_{33} & C_{34} & C_{35} & C_{36} \\
C_{41} & C_{42} & C_{43} & C_{44} & C_{45} & C_{46} \\
C_{51} & C_{52} & C_{53} & C_{54} & C_{55} & C_{56} \\
C_{61} & C_{62} & C_{63} & C_{64} & C_{65} & C_{66}
\end{array}\right]
$$

The number of independent elastic parameters reduces further with increasing degree of symmetry of the material. For example, plagioclase feldspar (monoclinic symmetry) requires all 21 elastic constants, olivine (orthorhombic symmetry) requires 9 elastic constant and quartz (hexagonal symmetry) requires 6 independent elastic constants.

The degree of anisotropy is given by the coefficient of anisotropy $k$ (Equation 2.11) [Stein and Wysession, 2003].

$$
k=\frac{V_{\text {max }}-V_{\text {min }}}{V_{\text {mean }}}
$$

In the Earth, seismic anisotropy can occur at various depths with varying length scales; thus, it can originate due to a number of reasons.

Anisotropy in the crust is mainly suggested to be due to differential stresses (stress-induced anisotropy) or the presence of structures (structure-controlled anisotropy). Symmetric combination of materials such as horizontally layered sedimentary rocks, sediments overlying layers of basalt and gabbro [Stein and Wysession, 2003] yields transverse anisotropy, with a vertically oriented axis of symmetry. This is also referred to as radial anisotropy where similar wave-speeds are observed along all azimuths in the horizontal plane and typically there is a slower wave speed along the vertical. Anisotropy in the crust can also occur as a result of the preferential closing of fluid-filled microcracks due to differential horizontal stresses [Babuška and Cara, 1991]. This can result in azimuthal anisotropy where seismic wave-speeds differ with azimuth.

Seismic anisotropy in the mantle is primarily due to the lattice preferred orientation 
(LPO) of intrinsically anisotropic minerals (e.g. olivine). Flow processes related to plate spreading can reorient olivine crystals parallel to the spreading direction, by slip along their [100] axes (Figure 2.7). P-wave propagation speed in olivine is fastest along this direction. Thus $\mathrm{P}_{\mathrm{n}}$ waves propagating along the spreading direction will show strong azimuthal anisotropy. Plate cooling with age will result in the freezing of the fabric responsible for this anisotropy and can be used to determine paleo-spreading directions of plates.

However, LPO of olivine crystals also depends on the pressure and temperature conditions and the presence of water [e.g. Jung and Karato, 2001; Karato et al., 2008]. Thus, the [100] axes of olivine crystals will not always be aligned along the direction of maximum shear stress. Several different types of olivine fabrics can be generated in response to these different conditions. In type-A fabric, the olivine [100] axis is subparallel to the shear direction and the (010) plane is subparallel to the shear plane. It typically occurs at low-stress and water-poor conditions. It is considered to be the cause of anisotropy in the oceanic lithosphere generated by stresses due to tectonic plate movement or stresses within the lithosphere [Karato et al., 2008]. Type-B fabric, where the olivine [001] axis is subparallel to the shear direction and the (010) plane is subparallel to the shear plane, occurs at high-stress (>300 MPa) and water-rich conditions such as at the corner of the mantle wedge in subduction zones. In type-C olivine fabric, the [001] axis is subparallel to the shear direction and the (100) plane is subparallel to the shear plane, and it occurs at low-stress and water-rich conditions [Karato et al., 2008]. Type-D and type-E olivine fabrics are similar to type-A, where the [100] axis subparallels the shear direction [Jung and Karato, 2001; Katayama et al., 2004]. Type-D fabric occurs in water-poor and high-stress conditions [Jung and Karato, 2001]. Type-E fabric occurs at moderate water content and low stress conditions [Katayama et al., 2004].

\subsubsection{Shear-wave Splitting}

Shear-wave splitting, also known as seismic birefringence, is a widely used technique to assess seismic anisotropy in the Earth [Savage, 1999]. It is based on the observation that when a shear-wave enters an anisotropic medium, it splits into two separate waves, one which travels faster and the other slower [Savage, 1999; Stein and Wysession, 2003]. The faster wave is polarised parallel to the fast direction and leads the orthogonal slow 


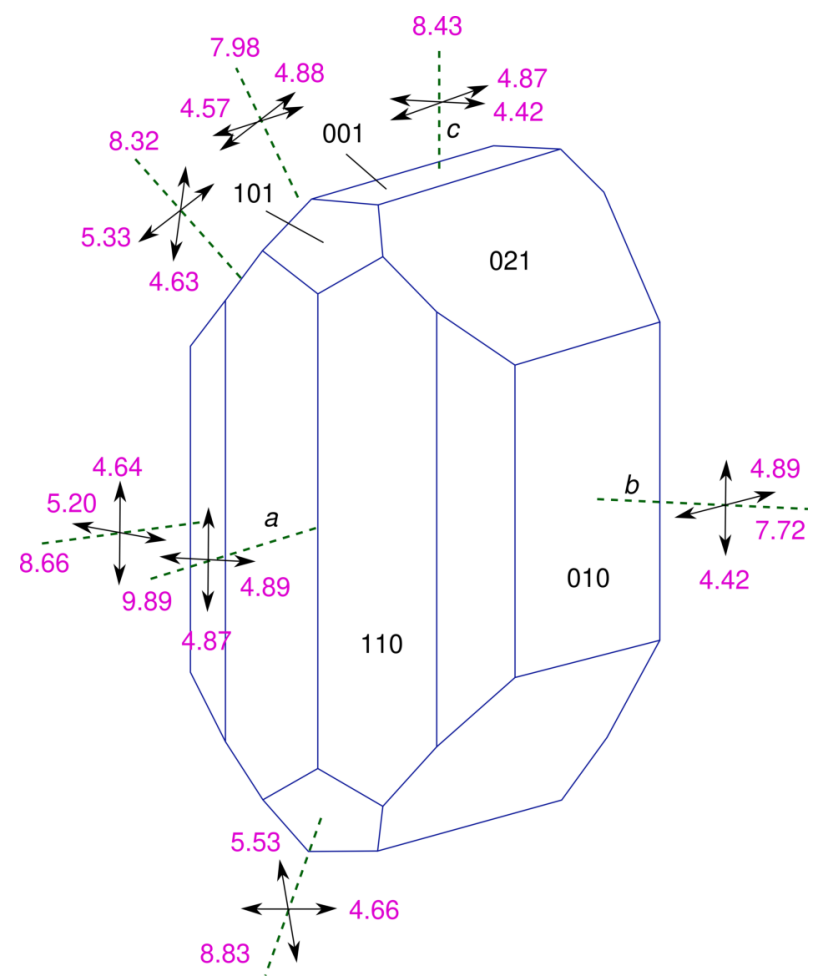

Figure 2.7 P- and S-wave velocities in $\mathrm{km} / \mathrm{s}$ along different crystallographic directions for olivine. Pwave velocities are along the directions of green dashed lines. S-wave velocities are along the two sets of perpendicular lines (black arrows) to the green dashed lines. Modified after Stein and Wysession [2003].

wave (Figure 2.8). Shear-wave splitting techniques can be used to calculate the fast polarisation direction $(\phi)$ and the delay time $(d t)$ (Figure 2.8), which can then be used to infer tectonic processes, such as deformation or state of stresses in the region.

Shear-wave splitting has been widely used with SKS phases of teleseismic earthquakes [Stein and Wysession, 2003] to assess the anisotropic nature of the upper mantle [Gledhill and Gubbins, 1996; Savage, 1999; Silver and Chan, 1988]. Crustal anisotropy has also been assessed in a variety of tectonic settings using S-waves from local earthquakes [e.g. Crampin, 1990; Graham et al., 2020; Savage et al., 2010; Zal et al., 2020].

Shear-wave splitting has also been used to measure anisotropy with PPS-type modeconverted phases in controlled-source seismic data in active margins [e.g. Kandilarov et al., 2015; Peacock et al., 2010; Pulford et al., 2003; Tsuji et al., 2011].

\subsection{Synthetic Waveform Modelling}

Although the geometric ray theory and modern raytracing techniques like RAYINVR provide convenient means of calculating travel-times of seismic waves between a source and a receiver to produce a velocity model, they do not use much of the 


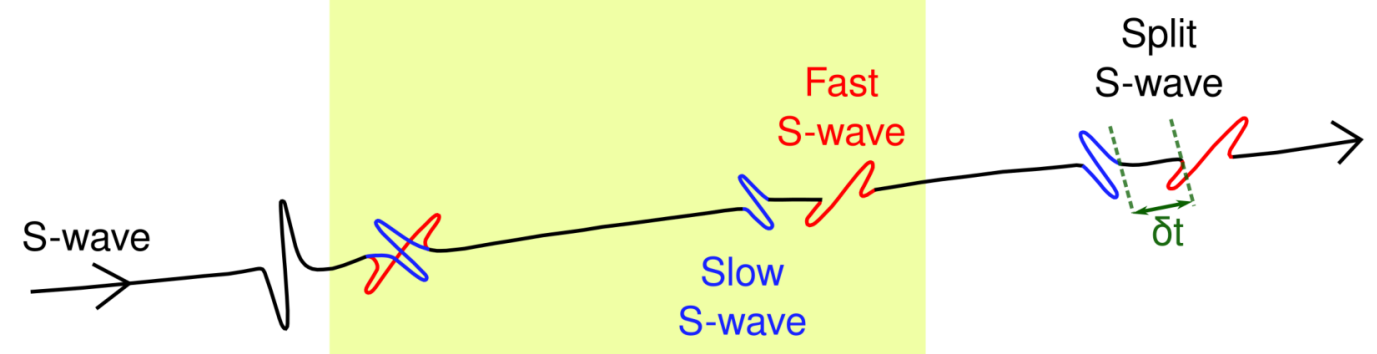

Figure 2.8 Schematic of shear wave splitting when a shear (S-) wave propagates through an anisotropic medium. $\delta t$ is the time delay accumulated between the fast and the slow $S$-waves due to the propagation through the anisotropic medium. Modified after Wüstefeld et al. [2008].

information carried inside the waveforms (e.g. frequency, amplitude). In wide-angle reflection and refraction modelling, it can sometimes be a challenge to explain some seismic phases purely from a travel-time point of view, as these arrivals can be rather enigmatic. Therefore, synthetic waveform modelling may be required to test different velocity models that can explain such phase arrivals. Furthermore, synthetic waveform modelling can be used to validate velocity models obtained from travel-time modelling.

In synthetic seismogram modelling, seismic wave propagation simulations are conducted based on an Earth model. In this, the seismic wave equation (Equation 2.12) is solved on a discretised Earth model. Ground displacements $(u)$ due to the propagation of the seismic waves from a source at receiver locations are calculated.

$$
\nabla^{2} u=\frac{1}{V^{2}} \frac{\partial^{2} u}{\partial t^{2}}
$$

Here, $\nabla^{2}=\frac{\partial^{2}}{\partial x^{2}}+\frac{\partial^{2}}{\partial y^{2}}+\frac{\partial^{2}}{\partial z^{2}}, u$ is the seismic wave-field, $V$ is the seismic wave velocity of the medium and $t$ is time.

A number of methods are available to calculate synthetic seismograms. They differ from each other on how the seismic wave equation is solved on the discretised earth 
model. These include the finite-difference method, pseudospectral method, finite-element method, finite-volume method and spectral-element method. Igel [2017] provides an overview on the theory, implementation and usage of these techniques.

The most widely used, conventional method is the finite-difference method which solves the strong form of the seismic wave equation (in differential form). However, it suffers difficulties in accurately modelling complex model boundaries (e.g. free surface) [Komatitsch and Tromp, 1999], and when incorporating anisotropy into the Earth models. Pseudospectral methods also suffer difficulties in handling free-surface boundary conditions and are limited to models with smooth variations [Komatitsch and Tromp, 1999]. Finite-element method can bypass some of the limitations in these methods as it can incorporate complex structures like surface topography or internal structures [Igel, 2017], but requires greater computational power to solve large systems of linear equations [Komatitsch and Tromp, 1999].

The spectral-element method, at present, is one of the widely applied numerical techniques for seismic wave propagation simulations which surpasses much of the limitations in the previously mentioned techniques.

\subsubsection{Spectral-element Method}

The spectral-element method is based on the finite-element method. It solves the weak form of the seismic wave equation (in integral form), on meshes of quadrangular elements in two-dimensional models or hexahedral elements in three-dimensional models, adapted to the free surface and to the main interfaces within the model [Komatitsch and Tromp, 1999]. In the weak form of the seismic wave equation, the stress-free boundary condition is naturally satisfied and does not need to be imposed explicitly. Anisotropy strain components can also be conveniently defined on the mesh element [Chen and Tromp, 2007]. The seismic wavefield on the elements is discretised using high-degree Lagrange interpolants, and integration over an element is accomplished based upon the Gauss-Lobatto-Legendre integration rule [Komatitsch and Tromp, 1999].

Free and open source codes which implement the spectral-element technique for seismic wave propagation simulations are the SES3D package 
(https://cos.ethz.ch/software/production/ses3d.html) and the SPECFEM package (https://github.com/SPECFEM). The SPECFEM package has been implemented as SPECFEM2D for two-dimensional seismic wave propagation simulation, SPECFEM3D Cartesian for three-dimensional seismic wave propagation simulation in regional scales and SPECFEM3D Globe for global scale seismic-wave propagation simulation in three-dimensions.

In this thesis, seismic wave propagation simulations are carried out using the SPECFEM2D package [Tromp et al., 2008].

\subsubsection{SPECFEM2D}

Since SPECFEM2D implements a spectral-element method, it can handle topography for layer boundaries (including the free-surface). It is also optimised to run in parallel using many CPUs in high performance computer systems, and in graphical processing units (GPUs) which cuts down the simulation time significantly [Komatitsch et al., 2010]. Most importantly, realistic velocity models which can include acoustic, elastic, anisotropic, poroelastic layers, or tomographic velocity models and attenuation can be used for the simulations. In addition, it can accurately simulate the amplitudes and phases of the reflections at the critical angle.

The user-manual, example input files for simulations and the source code of SPECFEM2D can be obtained from the COMPUTATIONAL INFRASTRUCTURE for GEODYNAMICS (CIG) community website (https://geodynamics.org/cig/software/specfem2d/).

\subsubsection{Mesh Generation}

Before, a seismic wave propagation simulation is carried out, the Earth model needs to be discretised. This is referred to as "meshing". Meshing creates a grid of spectral elements for the desired Earth model, honouring the free surface and the main interfaces in the model [Komatitsch and Tromp, 1999]. The size of a mesh element depends on several factors including the number of points per wavelength in the seismogram, P- and S- wave velocities in elastic media and P-wave velocity in acoustic media, and the dominant source frequency. Typically, for an accurate seismogram, $\sim 6$ points per wavelength is required. With SPECFEM, the number of points per wavelength in acoustic media is $\sim 5.5$, which is dependant on the minimum P-wave 
velocity of the medium. In elastic media, the number of points per wavelength is $\sim 5$ and is dependant on the minimum S-wave velocity [Komatitsch and Tromp, 1999]. Based on these criteria, the maximum size of an element in a mesh can be calculated using Equation 2.13.

$$
\text { Element Size }=\frac{\text { Minimum P- or S-wave Velocity }}{\text { Points per Wavelength } \times \text { Dominant Source Frequency }}
$$

Meshing for SPECFEM2D can be conducted using the inbuilt mesher xmeshfem2D, which creates a structured, quadrangular mesh based on the coordinates $(x, z)$ of the interfaces of the model, number of elements along the $x$-axis of the model and number of elements in between interfaces along the z-axis. External meshes from third party software like GMSH [Geuzaine and Remacle, 2009] can also be used. Special care should be taken to avoid twisting of the elements, i.e. the elements need to have modest curvature.

The mesh used in a simulation needs to be optimised based on the above parameters as large mesh elements will make the simulation unstable and will also not provide accurate results. Smaller mesh elements will make the models much larger making the computation expensive in terms of memory and time.

\subsubsection{Velocity and Density Model}

The velocity and density model for a simulation can consist of acoustic, elastic, poro-elastic, anisotropic and tomographic layers. To make the simulations simple and efficient, in this thesis, combinations of acoustic (isotropic), elastic (isotropic) and anisotropic layers are used. Acoustic layers are defined by their density and P-wave speed. Elastic layers are defined by their density, P- and S-wave speeds. Anisotropic layers are defined by the elastic tensor elements.

\subsubsection{Seismic Sources and Receivers}

In onshore-offshore and ocean bottom seismic experiments, a large number $(>1000)$ of airgun sources are recorded by onshore or ocean bottom seismographs. Generation of synthetic waveforms for a common receiver gather at a station with such a large number of sources is computationally expensive. Yet, generating synthetic waveforms for a single source at multiple receiver locations is simple. Therefore, for such 
simulations, the receiver is replaced by a source, and the airgun shots (sources) are replaced by a series of receivers as reciprocity is valid in synthetic waveform modelling [Igel, 2017].

The source is usually a "Ricker" point source [Shearer, 2009]. The fundamental frequency $\left(f_{0}\right)$ of the source is chosen so that the required resolution in the common receiver gathers will be achieved. The generated synthetic waveforms after the simulation can be convolved with the source-time function of the airgun source, so that the synthetic gather will have similar characteristics as the real common receiver gather.

\subsubsection{Time Step}

The time step $(\Delta t)$ is the interval at which the seismic wave-field is calculated. It should be chosen to be less than a threshold value so that the simulation will be stable and the resulting seismograms will have adequate resolution. The threshold value depends on the Courant number $(C)$ [Schuberth, 2003] and the minimum ratio between the distance $(h)$ of neighbouring mesh points and the wave-speeds $(v)$ in the model domain $(\Omega)$ (Equation 2.14).

$$
\Delta t<\operatorname{Cmin}_{\Omega}(h / v)
$$

This chapter provided the basic theoretical and methodological framework of the thesis. In addition to these, any different approaches and/or methods adopted are explained in Chapters 3, 4 and 5. 



\title{
Chapter 3
}

\section{Hydration of the Crust and Upper Mantle of the Hikurangi Plateau as it Subducts at the Southern Hikurangi Margin}

\begin{abstract}
Controlled-source seismic studies at most subduction zones show that bending of the subducting plate results in reduced seismic wave-speeds in the crust and upper mantle near the trench. Similar studies also have found unusually high P-wave-speeds $\left(\mathrm{V}_{\mathrm{P}}\right)$ in the upper mantle under oceanic plateaus. Onshore-offshore seismic profiling at the southern Hikurangi margin, where the $\sim 120$ Ma old oceanic Hikurangi Plateau is subducting, indicates that a fast $\left(\mathrm{V}_{\mathrm{P}} \sim 8.7 \pm 0.2 \mathrm{~km} / \mathrm{s}\right)$ upper mantle layer lies beneath a $\sim 25 \mathrm{~km}$ thick mantle layer with more regular wave-speeds $\left(\mathrm{V}_{\mathrm{P}} \sim 8.0 \pm 0.2 \mathrm{~km} / \mathrm{s}\right)$ under the Hikurangi trough. This is consistent with previous findings of upper mantle $\mathrm{V}_{\mathrm{P}} \sim 8.7-9.0 \mathrm{~km} / \mathrm{s}$ in the margin-parallel direction under the North Island ( $\sim 100 \mathrm{~km}$ northwest of the deformation front) at depths $\sim 8-10 \mathrm{~km}$ below the Moho. Our profiles are margin-perpendicular, thus we show that the upper mantle lid of the subducting Pacific Plate is characterised by unusually high P-wave-speeds along all azimuths. We find an area of lowered $V_{P}$ in the $\sim 12 \pm 1 \mathrm{~km}$ thick Hikurangi Plateau crust beneath the trough. This drop in $V_{P}$ is $\sim 10 \%$, and a similar drop in $V_{P}$ is deduced to depths of $25 \pm 2 \mathrm{~km}$ into the upper mantle. We interpret that the increase in thickness of the regular mantle beneath the trough results from the formation of a low-velocity zone in the faster upper mantle layer; this zone formed from serpentinisation by hydration through bending-induced normal faults and/or due to crack porosity introduced by thermal cracking, further enhanced by bending-related
\end{abstract}


faulting. Thus the "regular mantle" $\left(\mathrm{V}_{\mathrm{P}} \sim 8 \mathrm{~km} / \mathrm{s}\right)$ is not in fact regular, but rather the high-speed mantle has mechanically bent, fractured, and altered. The absolute depth of fast mantle $V_{\mathrm{P}}$ under the Hikurangi trough is around $50 \mathrm{~km}$. The onset of the lower band of seismicity of the double seismic zone and high upper mantle $V_{P}$ under the North Island is observed at similar depths. This is consistent with the hypothesis that the lower band of earthquakes in a double seismic zone is due to antigorite dehydration processes, a hydrous mineral formed in the low velocity zone in the upper mantle beneath the trough. Our study on the Hikurangi margin is different, as the subducting plate here contains a $\sim 120$ Ma old oceanic plateau with a $\sim 12 \mathrm{~km}$ thick crust, but the results are similar to other subduction margins where regular oceanic crust is subducting.

\subsection{Introduction}

Subduction zones have the capacity to recycle water back into the mantle [Cai et al., 2018; Ranero et al., 2003; Shillington et al., 2015] because bending of the subducting plate at the outer rise and trench creates avenues for hydration down to $\sim 30 \mathrm{~km}$ into the mantle [Cai et al., 2018; Emry and Wiens, 2015; Faccenda et al., 2009]. Seismic evidence from both passive and controlled-source studies show that a low-velocity region is created in the crust and upper mantle at the point of maximum bending in subduction zones. Controlled-source methods give more precise determination of wave-speed and suggest that there is a $\sim 10 \%$ drop in P-wave- speeds [e.g. Shillington et al., 2015; Van Avendonk et al., 2011]. On the other hand, passive-source seismic methods provide better depth estimates of the low-velocity region. Surface wave inversion results from the Mariana subduction zone reveal that the low wave-speed region is $\sim 30 \mathrm{~km}$ thick, $\sim 80 \mathrm{~km}$ long, pillow shaped, and roughly symmetrically disposed about the trench axis [Cai et al., 2018].

The reduction in seismic wave-speeds observed in the crust could be due to hydration and alteration of the gabbroic crust. In the upper mantle, it could be due to the serpentinisation of mantle peridotite by hydration along normal faults [Grevemeyer et al., 2018; Miller and Lizarralde, 2016] or the development of crack porosity further enhanced by bending-related faulting [Korenaga, 2017], although it is difficult to distinguish between the two. The concept of deep mantle hydration, whether by water filled cracks or hydrated minerals, is consistent with the hypothesis of antigorite 
dehydration being the source of the lower band of earthquakes in double seismic zones [Peacock, 2001], which are now considered a global feature for active margins [Brudzinski et al., 2007; Florez and Prieto, 2019]. From a global correlation of double seismic zones, a mean separation of $\sim 31 \mathrm{~km}$ is proposed to exist between the two bands of seismicity [Brudzinski et al., 2007; Florez and Prieto, 2019]. This is consistent with the proposition that mantle hydration due to bending can penetrate to depths of $30 \mathrm{~km}$ below the Moho [Cai et al., 2018; Emry and Wiens, 2015; Faccenda et al., 2009].

In this study, we present new evidence from controlled-source seismic data for a lowspeed zone in the crust and mantle of the Hikurangi Plateau, as it subducts beneath the North Island of New Zealand at the southern Hikurangi margin. The Hikurangi margin is different from other subduction margins where bending related hydration of the crust and upper mantle is suggested to occur (e.g. Alaska, middle America, Marianas, Northern Japan). The sedimentation rate at the Hikurangi margin is high, and the plate that is being subducted contains an oceanic plateau with a thick (10-12 $\mathrm{km}$ ) crust. Significant hydration of thick oceanic lithosphere is a good conduit to deliver water into the deep interior of the Earth. Thus, the importance of this study is to present a further case of plate-bending related reduced seismic wave-speeds in the subducting oceanic lithosphere, but in a different setting and under different conditions to other subduction zones.

\subsection{Hikurangi Subduction Margin}

The Hikurangi margin at the east coast of North Island, New Zealand, marks the subduction of the Pacific Plate under the Australian Plate (Figure 3.1). The subducting Pacific Plate here contains the oceanic Hikurangi Plateau (HP), a large igneous province. The HP is considered to have formed together with the Ontong-Java and Manihiki Plateaus as a single large super-plateau based on similar formation ages, chemical composition, velocity structure and submarine emplacement [Hochmuth et al., 2019; Taylor, 2006]. The original super-plateau, Ontong-Java-Manihiki-Hikurangi Plateau, is thought to have originated in the Lower Jurassic-Early Cretaceous as part of an oceanic spreading centre overlying a hotspot plume head [Hoernle et al., 2010]. In the Early Cretaceous, the Ontong-Java, Manihiki and Hikurangi plateaus rifted apart and were brought to their present positions by tectonic plate motions [Davy 
et al., 2008; Hochmuth et al., 2015; Taylor, 2006]. While the Ontong-Java Plateau is too buoyant to subduct [Miura et al., 2004], the HP subducts readily [Walcott, 1984].

Oceanic plateaus are a type of oceanic lithosphere that has undergone extensive melting and modification after formation at a mid-oceanic ridge [Coffin and Eldholm, 1994]. The melting is thought to be due to interaction with a mantle plume, although alternative explanations include melting due to a collision structure, large-scale Rayleigh-Taylor instability [Tanton and Hager, 2000] or decompression melting of buoyant mantle that was removed from a cratonic root by subduction [Mochizuki et al., 2019]. Because of this melting, the mantle peridotite becomes depleted and the overlying oceanic crust is thickened. Geophysical consequences of this process are that the plateau becomes more buoyant, because of both thickened crust and depleted mantle, and that the Pwave-speeds in the depleted mantle increase [Jordan, 1978].

An estimate of $\sim 15 \mathrm{~km}$ for the thickness of the HP crust was initially made for the southern Hikurangi margin from gravity modelling [Wood and Davy, 1994]. Recent, controlled-source seismic studies reveal a $\sim 10 \mathrm{~km}$ thick HP crust under the southwestern North Island [Henrys et al., 2013; Tozer et al., 2017] and $10 \mathrm{~km}$ thick crust overlain by a $\sim 1-2 \mathrm{~km}$ thick volcanoclastic layer offshore of the east coast of southern North Island [Mochizuki et al., 2019]. These are supported by receiver function studies suggesting a thickness of $\sim 11 \mathrm{~km}$ for the HP crust [Savage et al., 2007]. Bannister [1988] estimates a thickness of 11-12 km for the crust beneath Hawkes Bay (Figure 3.1a), based on relocated earthquake hypocentres. Reyners [2012], based on the distribution of seismicity, suggests that the crust of the HP is $\sim 35 \mathrm{~km}$ thick. Thus, there is some variation in interpretations for the thickness of HP crust.

Earthquake travel-time studies on the eastern North Island of New Zealand along northeast-southwest oriented raypaths infer high P-wave-speeds $\left(\mathrm{V}_{\mathrm{P}}\right)$ of 8.50-9.00 $\mathrm{km} / \mathrm{s}$ within the upper mantle of the subducting plate [Bannister, 1988; Galea, 1992; Haines, 1979; Kayal and Smith, 1984] (Figure A.1). These are all refracted waves in the upper mantle and can be referred to as $\mathrm{P}_{\mathrm{n}}$ [Storchak et al., 2011]. An active-source, wide-angle reflection and refraction experiment in the eastern North Island, also oriented northeast-southwest confirms the presence of a fast $P_{n}\left(V_{P}=8.70-8.80 \mathrm{~km} / \mathrm{s}\right)$ layer, and indicates that it is not directly below the Moho, but underlies a regular mantle layer of thickness $\sim 8-10 \mathrm{~km}$ and $\mathrm{V}_{\mathrm{P}}$ of $8.10-8.20 \mathrm{~km} / \mathrm{s}$ [Chadwick, 1997] (Figure 
A.1). All these studies measure $P_{n}$ in the margin-parallel direction. One explanation for the high upper-mantle $V_{P}$ is that they are a result of anisotropy created by flow-induced orientation of mantle olivine crystals [Brisbourne and Stuart, 1998; Galea, 1992; Robinson, 1986]. Shear-wave splitting of SKS phases have also yielded margin-parallel fast directions in this region [Marson-Pidgeon and Savage, 2004], but SKS phases cannot distinguish well what depths correspond to the anisotropy. A few passive-source seismic studies [Chong, 1982; Eberhart-Phillips et al., 2010; Robinson, 1986] and a controlled-source seismic study [Mochizuki et al., 2019] conducted along margin-perpendicular directions have also found the existence of fast $P_{n}$ speeds. Reyners [2012] suggests that the lower crust of the $\sim 35 \mathrm{~km}$ thick HP crust is transformed to eclogite to explain the high P-wave-speeds $(>8.5 \mathrm{~km} / \mathrm{s})$. Another recently proposed explanation is radial anisotropy created by a fabric specific to the gravitational collapse of a plume head [Stern et al., 2020]. Regardless of interpretation, the upper mantle of the $\mathrm{HP}$ has unusually high $\mathrm{V}_{\mathrm{P}}$, and these wave-speeds are azimuthally isotropic.

\subsection{Data}

During the Seismic Array HiKurangi Experiment (SAHKE) [Henrys et al., 2013], two transects of temporary seismographs were deployed north of the main SAHKE transect to record wide-angle reflections and refractions from the airgun shots of PEGASUS23 and PEGASUS25 offshore multi-channel seismic (MCS) lines (Figure 3.1b). The temporary seismographs (PEG1-PEG13) included L4 and L28 short-period, three-component geophone sensors connected to Reftek RT130 data loggers [Seward et al., 2010]. M/V Reflect Resolution acquired the PEGASUS and SAHKE MCS lines with a $10 \mathrm{~km}$ long streamer. The airgun source for PEGASUS lines had a capacity of 5,400 cubic inches and was shot every $37.5 \mathrm{~m}$. Both parameters were increased to 6,000 cubic inches and $100 \mathrm{~m}$, respectively, for SAHKE lines. The source was towed at a depth of $6 \pm 1 \mathrm{~m}$. The two transects are identified as PEGASUS23 and PEGASUS25 transects in this paper. Their onshore segments are defined by extending the two MCS lines towards the northwest along their azimuths (Section A.1.1 and Figure A.2). Origins of the model distance (x-axis) of the two transects are at the perpendicularly projected locations of the permanent seismic station MRZ and PRWZ (Figure $3.1 b$ ), respectively, to the two transects. Wide-angle reflections and refractions 
of the same airgun shots recorded on the permanent stations of the GeoNet and Regional seismic networks are also used in this study. Details about the seismographs used are given in Table A.1.

Larger source-receiver offsets are obtained by using the data recorded on the temporary and permanent stations with the airgun shots of the SAHKE01 line. SAHKE01 line deviates $10^{\circ}$ from the PEGASUS 23 and $15^{\circ}$ from the PEGASUS25 transects. An implicit assumption in interpreting these gathers, in terms of a 2D model, is that the crustal structure does not vary laterally beneath the triangular region defined by the shot line and all the direct raypaths to the onshore station (Figure 3.1b). We minimise this 3D to $2 \mathrm{D}$ projection issue by only using the shots that have source-receiver offsets greater than $150 \mathrm{~km}$ for MRZ, $100 \mathrm{~km}$ for PEG3, and $130 \mathrm{~km}$ for BFZ. The maximum error in velocity estimation due to this projection is $\sim 0.05 \mathrm{~km} / \mathrm{s}$ (Section A.1.2 and Figure A.3).

The main objective of using this onshore-offshore dataset is to constrain the velocity structure of the subducting Hikurangi Plateau. Offshore airgun source to onshore receiver raypaths traverse the crust and upper mantle across the Hikurangi trough. We focus on modelling travel-times of the observed refractions and wide-angle reflections to understand how the seismic velocities of the subducting oceanic crust and upper mantle are affected by bending of the plate near the trough.

\subsection{Velocity Modelling}

\subsubsection{Onshore-offshore Common Receiver Gathers}

Common receiver gathers at onshore seismographs were generated by extracting seismograms using GPS-based airgun shot times, from the continuously recorded data of the vertical component at each seismometer and plotting against shot offset. Twelve common receiver gathers were produced from the airgun shots of PEGASUS23 line (Figures 3.2a, A.4a-A.14a). Six receiver gathers were produced from the airgun shots of the PEGASUS25 line (Figures 3.3a, A.15a-A.19a). Three receiver gathers were produced from the airgun shots of the SAHKE01 line (Figures 3.4a, 3.5a, and A.20a). Bandpass filtering at 2-4-8-12 $\mathrm{Hz}$ corner frequencies and automatic gain control were applied to all the gathers. Finally, a reduction velocity was applied to distinguish between refracted seismic phases.

The identified seismic phases in the processed receiver gathers were: 


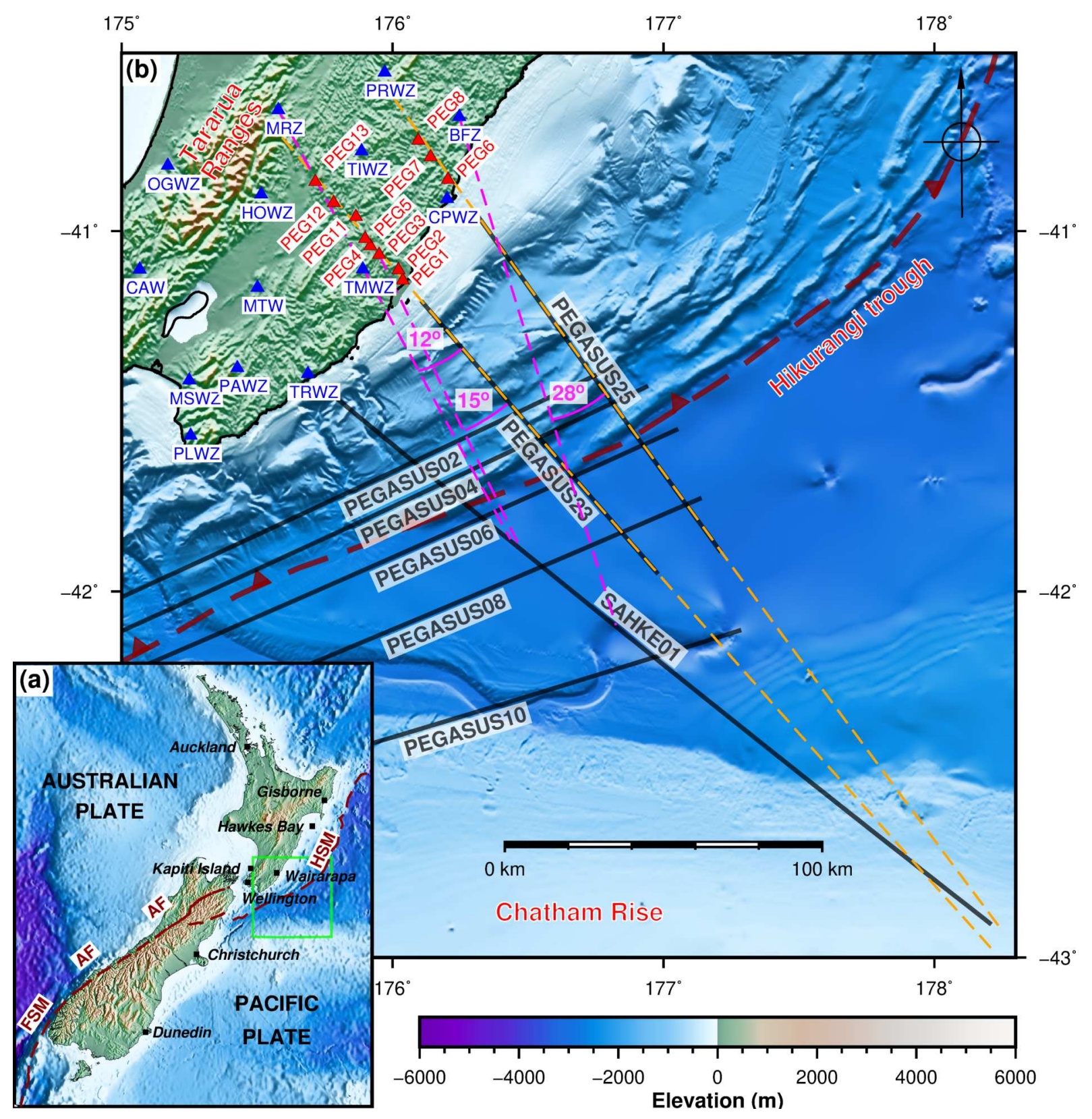

Figure 3.1 (a) Tectonic setting around New Zealand. HSM - Hikurangi Subduction Margin, AF - Alpine Fault, FSM - Fiordland Subduction Margin. Light green rectangle indicates the extent of (b). (b) Map of the study area. Black lines are multi-channel seismic (MCS) lines. Red triangles are temporary seismic stations. Blue triangles are permanent seismic stations. Dark red, dashed line is the Hikurangi trough. Orange dashed lines are extended onshore-offshore transects. Pink dashed lines connect airgun shots from the SAHKE01 line to MRZ, PEG3 and BFZ stations that have source-receiver offsets of 150, 100 and $160 \mathrm{~km}$, respectively. Only the shots with offsets greater than these source-receiver offsets (southeast of the intersection with the SAHKE01 line) have been used in the study. 
Hydration of the Crust and Upper Mantle of the Hikurangi Plateau as it Subducts at the Southern HiKURANGi MARGIN
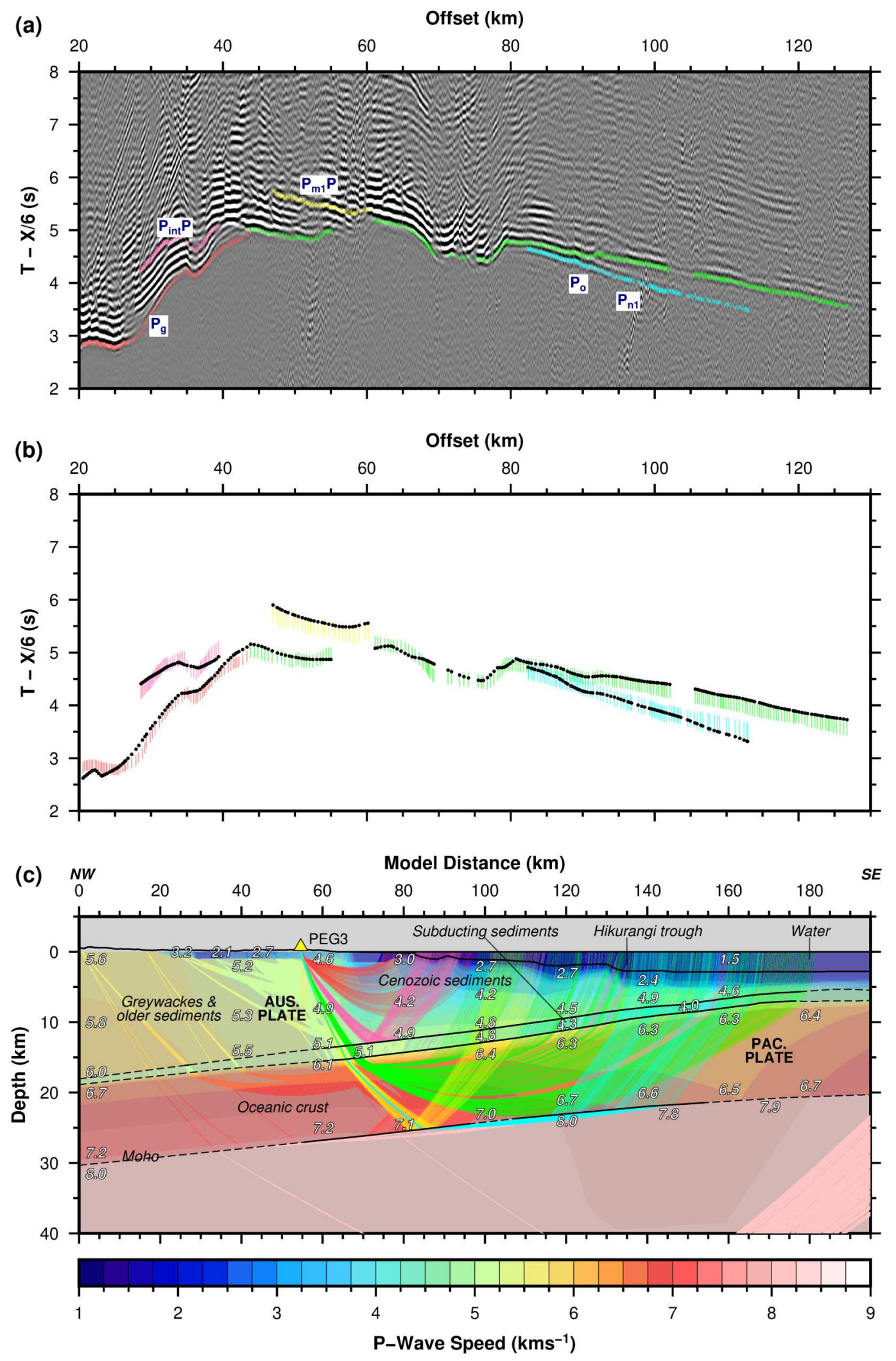

Figure 3.2 (a) Common receiver gather at PEG3 station with airgun shots of PEGASUS23 line. Different seismic phases are colour-coded. $\mathrm{P}_{\mathrm{g}}$-red, $\mathrm{P}_{\text {int }} \mathrm{P}$-pink, $\mathrm{P}_{\mathrm{o}}$-light green, $\mathrm{P}_{\mathrm{m} 1} \mathrm{P}$-yellow and $\mathrm{P}_{\mathrm{n} 1}$-cyan. (b) Observed travel-times (colour-coded as in (a)) and calculated travel-times (black dots). The height of an observed travel-time pick is twice its pick uncertainty. Note that the travel-time axes of (a) and (b) are reduced at $6 \mathrm{~km} / \mathrm{s}$. (c) Traced rays and the velocity model, with rays colour-coded as in (a) and (b). Yellow triangle-location of seismograph. Grey shading indicates areas in the velocity model without ray coverage. White numbers with black outline are P-wave-speeds at those locations in $\mathrm{km} / \mathrm{s}$. Dashed black lines are inferred interfaces. Continuous black lines are confirmed interfaces. The x-axes in (a) and (b) indicate the offset from the recording station and the $x$-axis in (c) indicates the model distance starting from MRZ station. AUS. PLATE and PAC. PLATE are Australian Plate and Pacific Plate respectively. 
(a)

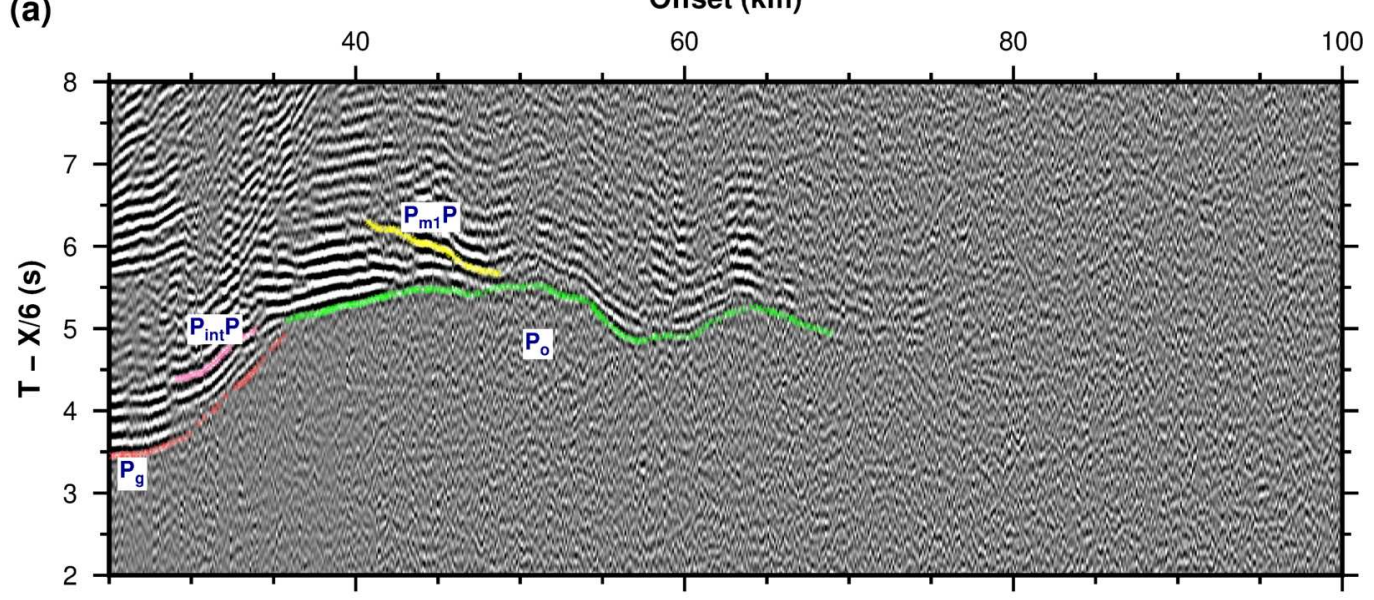

(b)
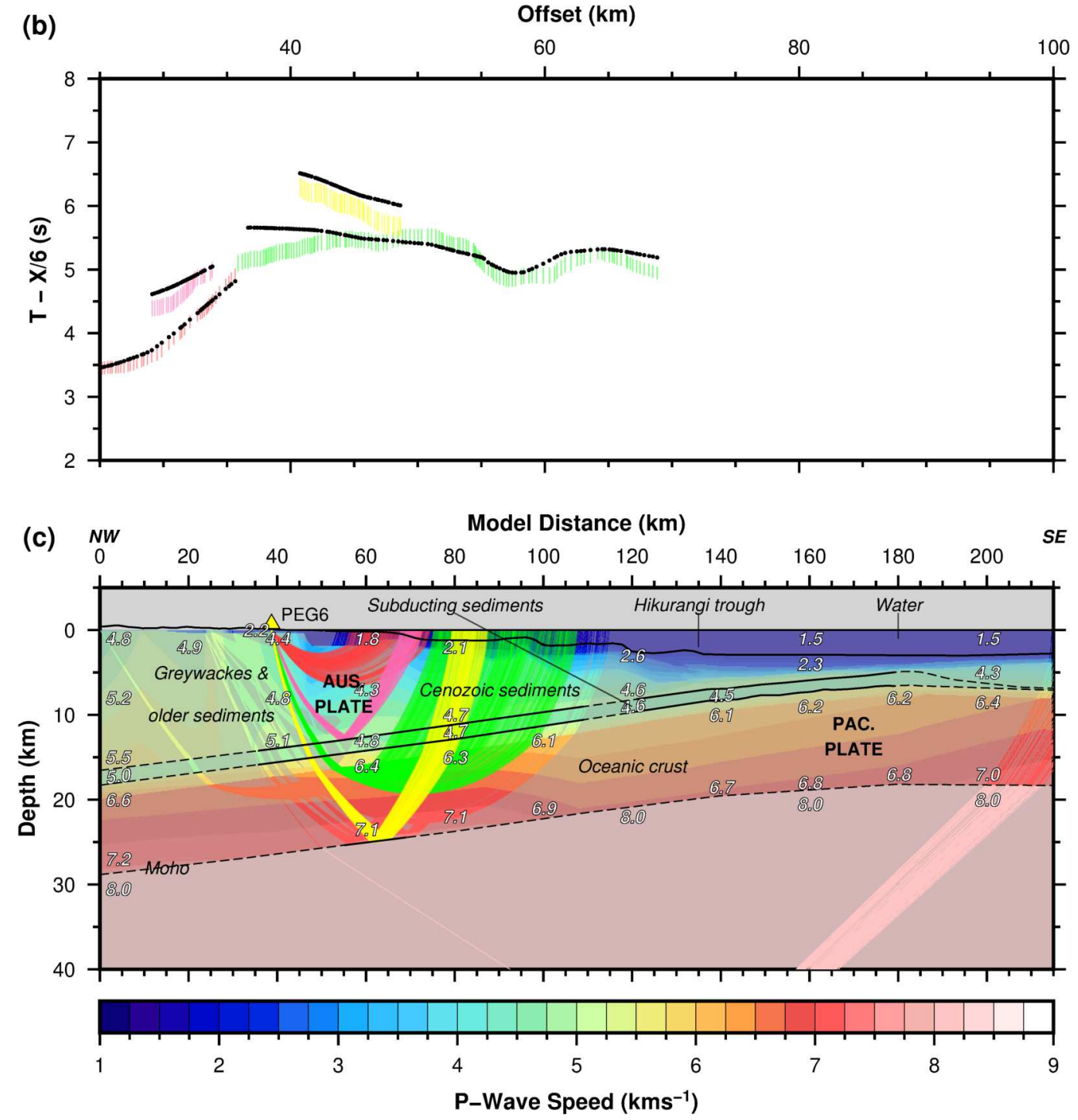

Figure 3.3 (a) Common receiver gather at PEG6 station with airgun shots of PEGASUS25 line. Different seismic phases are colour-coded. $\mathrm{P}_{\mathrm{g}}$-red, $\mathrm{P}_{\text {int }} \mathrm{P}$-pink, $\mathrm{P}_{\mathrm{o}}$-light green, $\mathrm{P}_{\mathrm{m} 1} \mathrm{P}$-yellow and $\mathrm{P}_{\mathrm{n} 1}$-cyan. (b) Observed travel-times (colour-coded as in (a)) and calculated travel-times (black dots). The height of an observed travel-time pick is twice its pick uncertainty. Note that the travel-time axes of (a) and (b) are reduced at $6 \mathrm{~km} / \mathrm{s}$. (c) Traced rays and the velocity model, with rays colour-coded as in (a) and (b). Yellow triangle-location of seismograph. Grey shading indicates areas in the velocity model without ray coverage. White numbers with black outline are P-wave-speeds at those locations in $\mathrm{km} / \mathrm{s}$. Dashed black lines are inferred interfaces. Continuous black lines are confirmed interfaces. The x-axes in (a) and (b) indicate the offset from the recording station and the x-axis in (c) indicates the model distance starting from MRZ station. AUS. PLATE and PAC. PLATE are Australian Plate and Pacific Plate respectively. 
Hydration of the Crust and Upper Mantle of the Hikurangi Plateau as it Subducts at the Southern HiKURANGi MARgin
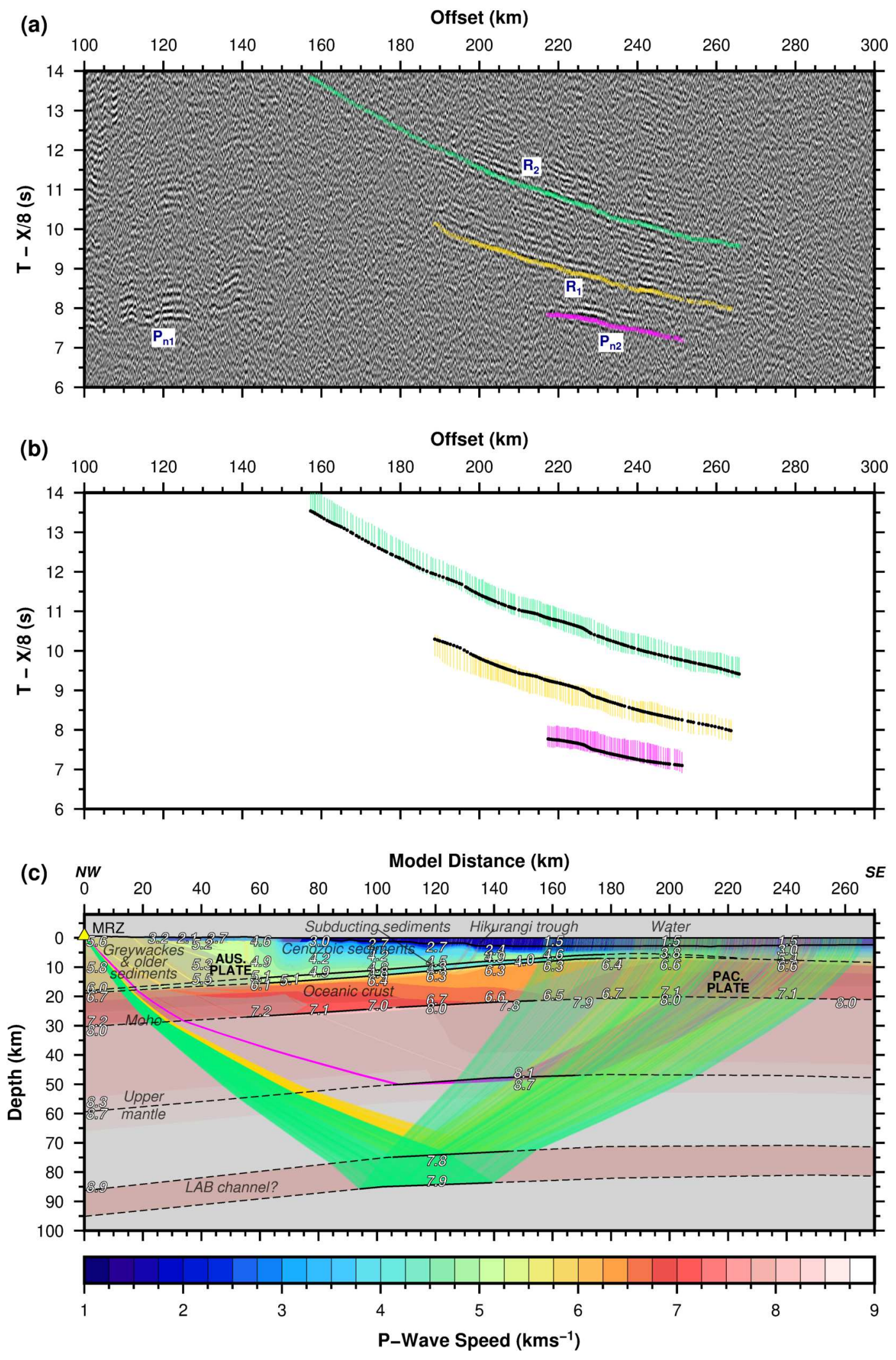

Figure 3.4 (a) Common receiver gather at MRZ station with airgun shots of SAHKE01 line. Different seismic phases are colour-coded. $\mathrm{P}_{\mathrm{g}}$-red, $\mathrm{P}_{\text {int }} \mathrm{P}$-pink, $\mathrm{P}_{\mathrm{o}}$-light green, $\mathrm{P}_{\mathrm{m} 1} \mathrm{P}$-yellow and $\mathrm{P}_{\mathrm{n} 1}$-cyan. (b) Observed travel-times (colour-coded as in (a)) and calculated travel-times (black dots). The height of an observed travel-time pick is twice its pick uncertainty. Note that the travel-time axes of (a) and (b) are reduced at $6 \mathrm{~km} / \mathrm{s}$. (c) Traced rays and the velocity model, with rays colour-coded as in (a) and (b). Yellow triangle-location of seismograph. Grey shading indicates areas in the velocity model without ray coverage. White numbers with black outline are P-wave-speeds at those locations in $\mathrm{km} / \mathrm{s}$. Dashed black lines are inferred interfaces. Continuous black lines are confirmed interfaces. The x-axes in (a) and (b) indicate the offset from the recording station and the $x$-axis in (c) indicates the model distance starting from MRZ station. AUS. PLATE and PAC. PLATE are Australian Plate and Pacific Plate respectively. 

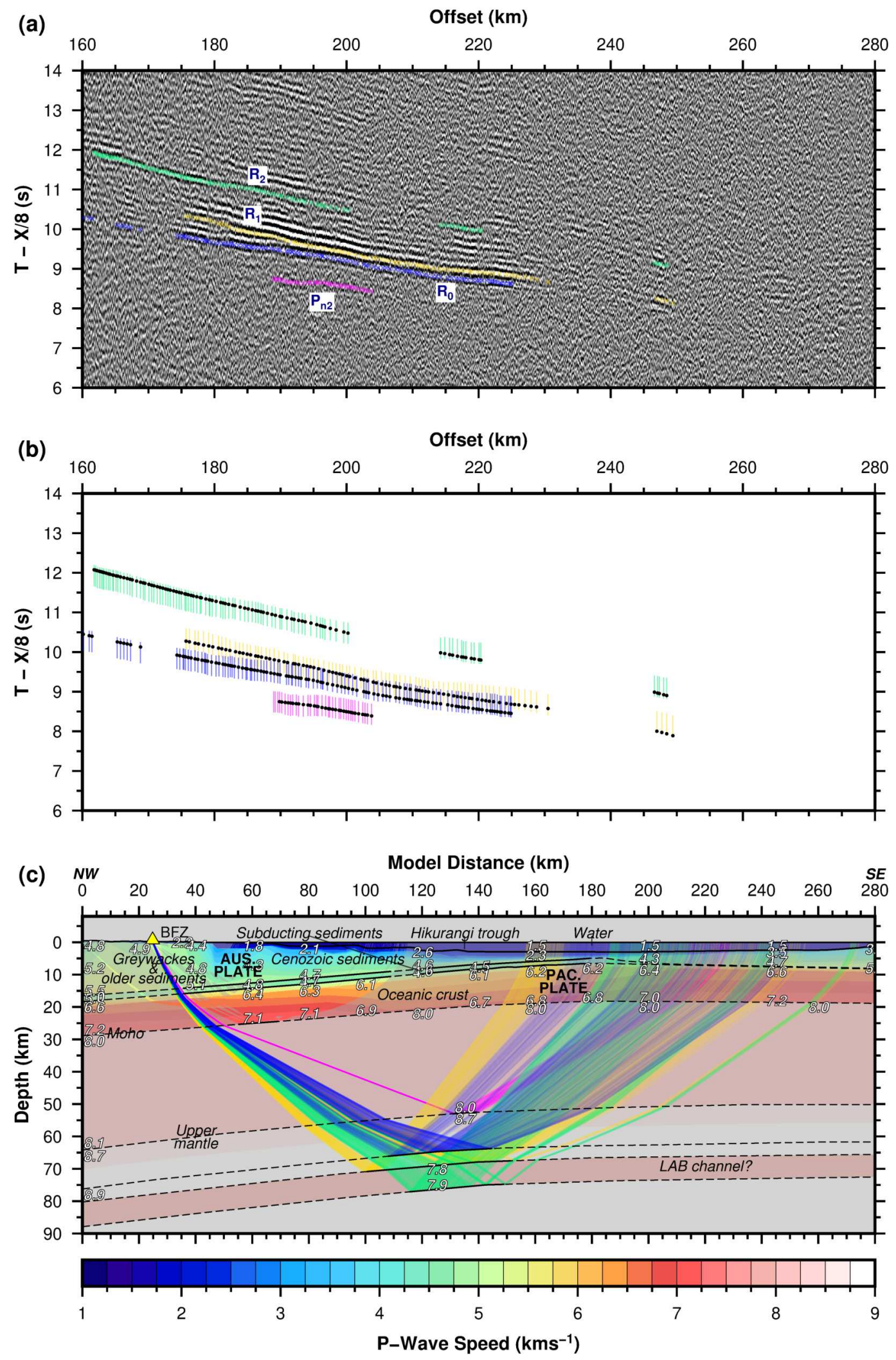

Figure 3.5 (a) Common receiver gather at BFZ station with airgun shots of SAHKE01 line. Different seismic phases are colour-coded. $\mathrm{P}_{\mathrm{g}}$-red, $\mathrm{P}_{\text {int }} \mathrm{P}$-pink, $\mathrm{P}_{\mathrm{o}}$-light green, $\mathrm{P}_{\mathrm{m} 1} \mathrm{P}$-yellow and $\mathrm{P}_{\mathrm{n} 1}$-cyan. (b) Observed travel-times (colour-coded as in (a)) and calculated travel-times (black dots). The height of an observed travel-time pick is twice its pick uncertainty. Note that the travel-time axes of (a) and (b) are reduced at $6 \mathrm{~km} / \mathrm{s}$. (c) Traced rays and the velocity model, with rays colour-coded as in (a) and (b). Yellow triangle-location of seismograph. Grey shading indicates areas in the velocity model without ray coverage. White numbers with black outline are P-wave-speeds at those locations in $\mathrm{km} / \mathrm{s}$. Dashed black lines are inferred interfaces. Continuous black lines are confirmed interfaces. The x-axes in (a) and (b) indicate the offset from the recording station and the $x$-axis in (c) indicates the model distance starting from MRZ station. AUS. PLATE and PAC. PLATE are Australian Plate and Pacific Plate respectively. 
- $\mathrm{P}_{\mathrm{g}}$ - refraction in the crust of the overriding Australian Plate

- $\mathrm{P}_{\text {int }} \mathrm{P}$ - reflection from the plate interface that separates the overlying Australian Plate from the top of subducting sediments

- $\mathrm{P}_{\mathrm{o}}$ - refraction in the subducting $\mathrm{HP}$ crust

- $\mathrm{P}_{\mathrm{m} 1} \mathrm{P}$ - reflection from the Moho

- $\mathrm{P}_{\mathrm{n} 1}$ - refraction from the mantle beneath the Moho

- $\mathrm{P}_{\mathrm{n} 2}$-refraction from deeper upper mantle layer beneath the Moho of HP

In addition, we note three wide-angle reflections, $R_{0}, R_{1}$ and $R_{2}$ (Figures 3.4a, 3.5a and A.14a), with two-way travel-times $\sim 35-40$ sec.

\subsubsection{Initial Models}

A crucial step in this type of single-sided, onshore-offshore seismic surveys is to incorporate constraints for the near-surface seismic velocities in the onshore region because very low seismic velocities at the surface can propagate large travel-time misfits that will be compensated by the deeper structure.

Seismic [Cape et al., 1990; Chadwick, 1997] and gravity [Pancha, 1994] studies in the eastern Wairarapa region indicate the presence of low $V_{\mathrm{P}}(\sim 2.5 \mathrm{~km} / \mathrm{s})$ and thick $(\sim 1$ $\mathrm{km}$ ) sediments at the surface. We incorporated these constraints into the onshore sections of the initial velocity models for the two transects. Topography of the onshore sections of the models was obtained from [Mitchell et al., 2012].

In the offshore regions of the two transects, initial velocity models were constrained using MCS reflection data [Bland et al., 2015]. On the PEGASUS23 transect, MCS reflection data constrain the bathymetry between $\sim 70$ and $\sim 190 \mathrm{~km}$ model distances (Figure A.21). The top of a thin layer of low-velocity subducted sediments and the top of the Hikurangi Plateau crust are constrained between $\sim 130$ and $\sim 190 \mathrm{~km}$ model distances (Figure A.21). In the PEGASUS25 transect, the MCS data constrained the same layers between $\sim 60$ and $\sim 180 \mathrm{~km}$ and $\sim 120$ and $\sim 180 \mathrm{~km}$ model distances respectively (Figure A.22). These reflectors were picked in the time-domain and were depth converted using the stacking velocities (Figures A.21 and A.22). 
P-wave velocities of the initial velocity models used stacking velocities derived from the processing of MCS data (courtesy of Crown Minerals). The derivation of stacking velocities from MCS data assumes that the reflectors are horizontal. However, in the case of an MCS line along the dip of a subduction zone where the reflectors are dipping, velocity analysis may result in anomalous and unrealistic velocities. Accordingly, we use stacking velocities only from locations with dips $<2^{\circ}$.

In extending the two transects to accommodate the long-offset data from the SAHKE01 line, the tops of both the subducting sediments and the oceanic crust observed on the SAHKE01 MCS line (Figure A.23) and on the cross lines PEGASUS 2, 4, 6, 8 and 10 (Figure 3.1b) were picked and were converted to depths using the stacking velocities of those lines. Thereafter, these depths were linearly interpolated into the southeast extensions of the two transects. P-wave-speeds were directly projected from the stacking velocities of SAHKE01 (Figure A.23) to these extensions. Bathymetry of the extensions was obtained from Mitchell et al. [2012].

These constraints were incorporated to build initial velocity models for the two transects in RAYINVR [Zelt and Smith, 1992] format. RAYINVR velocity models are parametrised by layer boundaries defined by boundary nodes and the velocities within each layer are specified by upper and lower velocity nodes. This parametrisation conveniently introduces both vertical and horizontal velocity gradients in the velocity model.

The accuracy of the initial RAYINVR models was tested against the MCS reflection data by comparing the observed two-way travel-times of the prominent reflections in MCS data (Figures A.21 and A.22) with the calculated two-way travel-times for the same reflections in the RAYINVR models. The overall RMS of travel-time residuals for the two-way travel-times between the initial velocity model and the actual MCS data were $\sim 50 \mathrm{msec}$ and $\sim 70 \mathrm{msec}$ for the PEGASUS23 and PEGASUS25 transects, respectively.

\subsubsection{Forward-model Raytracing}

The travel-times of the observed phases were picked. Travel-time pick errors were assigned for each phase based on their dominant frequency, source-receiver offset and signal-to-noise ratio (Table 3.1).

The observed phases were raytraced using RAYINVR [Zelt and Smith, 1992] in a 
Table 3.1 Observed seismic phases in the common receiver gathers along PEGASUS23 and PEGASUS25 transects

\begin{tabular}{lcccccccc}
\hline \multirow{2}{*}{ Phase } & \multicolumn{4}{c}{ PEGASUS23 } & \multicolumn{5}{c}{ PEGASUS25 } \\
\cline { 2 - 9 } & $\mathbf{N}$ & Av. Unc. & $\mathrm{T}_{\text {RMS }}$ & $\chi^{2}$ & $\mathbf{N}$ & Av. Unc. & $\mathbf{T}_{\text {RMS }}$ & $\chi^{2}$ \\
\hline $\mathrm{P}_{\mathrm{g}}$ & 677 & 0.102 & 0.132 & 1.628 & 325 & 0.100 & 0.105 & 1.103 \\
$\mathrm{P}_{\mathrm{int}} \mathrm{P}$ & 417 & 0.112 & 0.137 & 1.613 & 138 & 0.125 & 0.205 & 2.705 \\
$\mathrm{P}_{\mathrm{o}}$ & 724 & 0.125 & 0.146 & 1.466 & 597 & 0.126 & 0.164 & 1.783 \\
$\mathrm{P}_{\mathrm{m} 1} \mathrm{P}$ & 195 & 0.143 & 0.223 & 3.246 & 152 & 0.150 & 0.217 & 2.104 \\
$\mathrm{P}_{\mathrm{n} 1}$ & 210 & 0.152 & 0.145 & 0.927 & na & na & na & na \\
$\mathrm{P}_{\mathrm{n} 2}$ & 118 & 0.200 & 0.137 & 0.473 & 33 & 0.200 & 0.047 & 0.056 \\
$\mathrm{R}_{0}$ & na & na & na & na & 137 & 0.200 & 0.122 & 0.374 \\
$\mathrm{R}_{1}$ & 165 & 0.200 & 0.134 & 0.450 & 122 & 0.200 & 0.084 & 0.177 \\
$\mathrm{R}_{2}$ & 225 & 0.200 & 0.179 & 0.808 & 100 & 0.200 & 0.128 & 0.413 \\
Overall & 2731 & na & 0.150 & 1.452 & 1604 & na & 0.155 & 1.463 \\
\hline $\mathrm{N}$ & & &
\end{tabular}

$\mathrm{N}=$ Number of travel-time picks.

Av. Unc. = Average uncertainty in travel-time picks of a phase in seconds.

$\mathrm{T}_{\mathrm{RMS}}=$ Normalised root mean square of travel-time misfits in seconds.

forward modelling approach to minimise the travel-time residuals. A forward modelling approach was favoured because of (1) the asymmetric source-receiver geometry of the onshore-offshore technique; (2) the sparse ray coverage in some areas of the models and (3) the ability to include constraints on wave-speeds and interface depths from previous studies [e.g. Tozer et al., 2017]. The top layers were modelled first using phases that sample them, and the deeper layers were modelled sequentially using latter phases to minimise the propagation of errors and ambiguities into the deeper layers.

\subsubsection{Model Parameter Uncertainties}

Perturbation tests were carried out sequentially from the top to the bottom of the model to assess the uncertainties in the determined velocities and thicknesses of each layer in the final preferred models (Figure 3.6). For a layer, depth nodes of the lower boundary and the velocity nodes at the top and/or bottom of the layer were perturbed at regular intervals with both positive and negative perturbations around the preferred values (Table 3.2). $\mathrm{T}_{\mathrm{RMS}}$ for the depth, velocity perturbation pair was obtained from RAYINVR and was plotted against the two parameters being perturbed 
(Figure 3.7). Model parameters that can trace the greatest number of observed travel-time picks with minimum $\mathrm{T}_{\mathrm{RMS}}$ were considered to best fit the actual model.

Variations in pick error are ignored and the errors ( $\left.t_{\text {observed }}-t_{\text {calculated }}\right)$ are assumed to be independent and to have a normal distribution with zero mean and common variance $\sigma^{2}$. The minimum $\mathrm{T}_{\mathrm{RMS}}{ }^{2}$ is then an estimate of $\sigma^{2}$ and non-optimal $\mathrm{T}_{\mathrm{RMS}}{ }^{2} /$ minimum $\mathrm{T}_{\mathrm{RMS}}{ }^{2}$ has approximately a $\chi^{2} / d f$ distribution, with $d f$ being the degrees of freedom $(d f$ $=$ no. of travel-time picks - no.of model parameters) [Stern and Benson, 2011]. The test with the lowest number of travel-time picks (Figure 3.7c, d) used 118 travel-time picks and 2 model parameters $(d f=116)$. We calculate the $\mathrm{T}_{\mathrm{RMS}}$ contour within which there is a $95 \%$ probability for the model parameter combination to fit the observations. This contour is referred as the $95 \%$ confidence interval $\left(\mathrm{T}_{\mathrm{RMS}, 95}\right)$ and is calculated using Equation 3.1.

$$
\mathrm{T}_{\mathrm{RMS}, 95}=\mathrm{T}_{\mathrm{RMS}, \min } \sqrt{\frac{\chi^{2}(0.95)}{d f}}
$$

We employed two parameter perturbation tests to estimate the uncertainties in the (1) thickness of the overriding Australian Plate crust and $V_{P}$ at its base (i.e. at the plate interface) (Figure A.24a), (2) $\mathrm{V}_{\mathrm{P}}$ at the base of the Australian Plate crust and in the dip of the plate interface (Figure A.24b), (3) thickness and $V_{P}$ of the Hikurangi Plateau crust (Figure 3.7a) and (4) thickness and $V_{P}$ of the regular mantle (Figure 3.7b). Also, uncertainties in the depth to the faster upper mantle layer and its $V_{P}$ have been estimated using a grid-search approach similar to perturbation tests mentioned earlier (Figure 3.7c, d). We used a three-parameter grid-search test to estimate the trade-offs between the $V_{P}$ at the base of the regular mantle layer, depth and $V_{P}$ of the faster upper mantle layer (Figure A.25). All model parameter uncertainties are given in Table 3.2.

Furthermore, we applied a sensitivity test to check the robustness of $V_{P}$ determination of the oceanic crust with offset (Figure 3.7e).

\subsection{Results}

Both PEGASUS23 and PEGASUS25 transects exhibit a similar crustal and upper mantle structure in the region sampled by the seismic waves (Figure 3.6). The root 
Hydration of the Crust and Upper Mantle of the Hikurangi Plateau as it Subducts at the Southern HikURANGI Margin

Table 3.2 Details of perturbation and grid-search tests to estimate model parameter uncertainties

\begin{tabular}{|c|c|c|c|c|}
\hline $\begin{array}{l}\text { Test Type } \\
\text { (Figure) }\end{array}$ & Model Parameter & Preferred Value & Min./Max./Inc. & Uncertainty \\
\hline Perturbation & Thickness of HP crust & $12 \mathrm{~km}$ & $-5 / 5 / 0.1 \mathrm{~km}$ & $\pm 1 \mathrm{~km}$ \\
\hline (Figure 3.7a) & $V_{P}$ of $H P$ crust & Variable & $-0.5 / 0.5 / 0.1 \mathrm{~km} / \mathrm{s}$ & $\pm 0.1 \mathrm{~km} / \mathrm{s}$ \\
\hline Perturbation & Thickness of HP regular mantle & $25 \mathrm{~km}$ & $-20 / 10 / 1 \mathrm{~km}$ & $\pm 2 \mathrm{~km}$ \\
\hline (Figure 3.7b) & $\mathrm{V}_{\mathrm{P}}$ of regular mantle & $8.0 \mathrm{~km} / \mathrm{s}$ & $-0.5 / 0.5 / 0.05 \mathrm{~km} / \mathrm{s}$ & $\pm 0.2 \mathrm{~km} / \mathrm{s}$ \\
\hline Grid-search & Depth to faster upper mantle from Moho & $25 \mathrm{~km}$ & $0 / 45 / 1 \mathrm{~km}$ & $\pm 2 \mathrm{~km}$ \\
\hline (Figure 3.7c) & $V_{P}$ at the top of faster mantle layer & $8.7 \mathrm{~km} / \mathrm{s}$ & $8.2 / 9.2 / 0.05 \mathrm{~km} / \mathrm{s}$ & $\pm 0.2 \mathrm{~km} / \mathrm{s}$ \\
\hline Perturbation & Thickness of Aus. plate cont. crust & Variable & $-2 / 2 / 0.1 \mathrm{~km}$ & $\pm 1 \mathrm{~km}$ \\
\hline (Figure A.24a) & Base $V_{P}$ of Aus. Plate cont. crust & Variable & $-0.5 / 0.5 / 0.05 \mathrm{~km} / \mathrm{s}$ & $\pm 0.1 \mathrm{~km} / \mathrm{s}$ \\
\hline Perturbation & Dip of the plate interface & $4^{\circ}$ & $-2.2^{\circ} / 2.2^{\circ} / 0.04^{\circ}$ & $\pm 1^{\circ}$ \\
\hline (Figure A.24b) & Base $V_{P}$ of Aus. Plate cont. crust & Variable & $-0.5 / 0.5 / 0.05 \mathrm{~km} / \mathrm{s}$ & $\pm 0.1 \mathrm{~km} / \mathrm{s}$ \\
\hline \multirow{3}{*}{$\begin{array}{l}\text { Grid-search } \\
\text { (Figure A.25) }\end{array}$} & $V_{P}$ at the base of regular mantle & $8.0 \mathrm{~km} / \mathrm{s}$ & $7.8 / 9.0 / 0.1 \mathrm{~km} / \mathrm{s}$ & $\pm 0.2 \mathrm{~km} / \mathrm{s}$ \\
\hline & Depth to faster upper mantle from Moho & $25 \mathrm{~km}$ & $0 / 40 / 1 \mathrm{~km}$ & $\pm 2 \mathrm{~km}$ \\
\hline & $V_{P}$ at the top of faster mantle & $8.7 \mathrm{~km} / \mathrm{s}$ & $8.0 / 9.2 / 0.1 \mathrm{~km} / \mathrm{s}$ & $\pm 0.2 \mathrm{~km} / \mathrm{s}$ \\
\hline
\end{tabular}

mean squared travel-time misfits $\left(\mathrm{T}_{\mathrm{RMS}}\right)$ and the $\chi^{2}$ statistics for the final preferred velocity models are given in Table 3.1. Travel-time misfits with model distance and histograms of travel-time misfits for the two transects are shown in Figures A.26 and A.27, respectively. Figures A.28 and A.29 show the ray density along the two transects.

\subsubsection{Crustal Structure of Overriding Australian Plate}

A P-wave-speed of $\sim 5.5 \mathrm{~km} / \mathrm{s}$ just below the weathering layer (Figure 3.6c) in the onshore crustal regions reflects the presence of consolidated sediments and Cretaceous basement greywacke in this region [Hines et al., 2013]. The speed increases to $\sim 6.0 \pm 0.1 \mathrm{~km} / \mathrm{s}$ at a depth of $\sim 18 \mathrm{~km}$. At shallow depths $(\sim 7 \mathrm{~km})$, an abrupt lateral change in $\mathrm{V}_{\mathrm{P}}$ from $\sim 5 \mathrm{~km} / \mathrm{s}$ to $\sim 4 \mathrm{~km} / \mathrm{s}$ can be observed at $\sim 70 \mathrm{~km}$ model distance (Figure 3.6), marking the transition from the greywackes to Cenozoic sediments. The depth to the plate interface at the coastline ( $\sim 60 \mathrm{~km}$ PEGASUS23 model distances) is $\sim 13 \mathrm{~km}$ and it reduces to $\sim 5 \mathrm{~km}$ at the Hikurangi trough (Figure 3.6a). The velocities along the base of the Australian Plate crust (i.e. at the plate interface) have been determined within $\pm 0.1 \mathrm{~km} / \mathrm{s}$ (Figure A.24a). The dip of the plate interface is $\sim 4^{\circ} \pm 1^{\circ}$ towards the west (Figure A.24b). Along PEGASUS23 transect, within its ray coverage, the plate interface is comparable to that of Williams et al. [2013], who derived the plate interface model by interpolating the upper envelope of relocated earthquakes in the subducting slab and the plate interface identified by active-source seismic surveys 


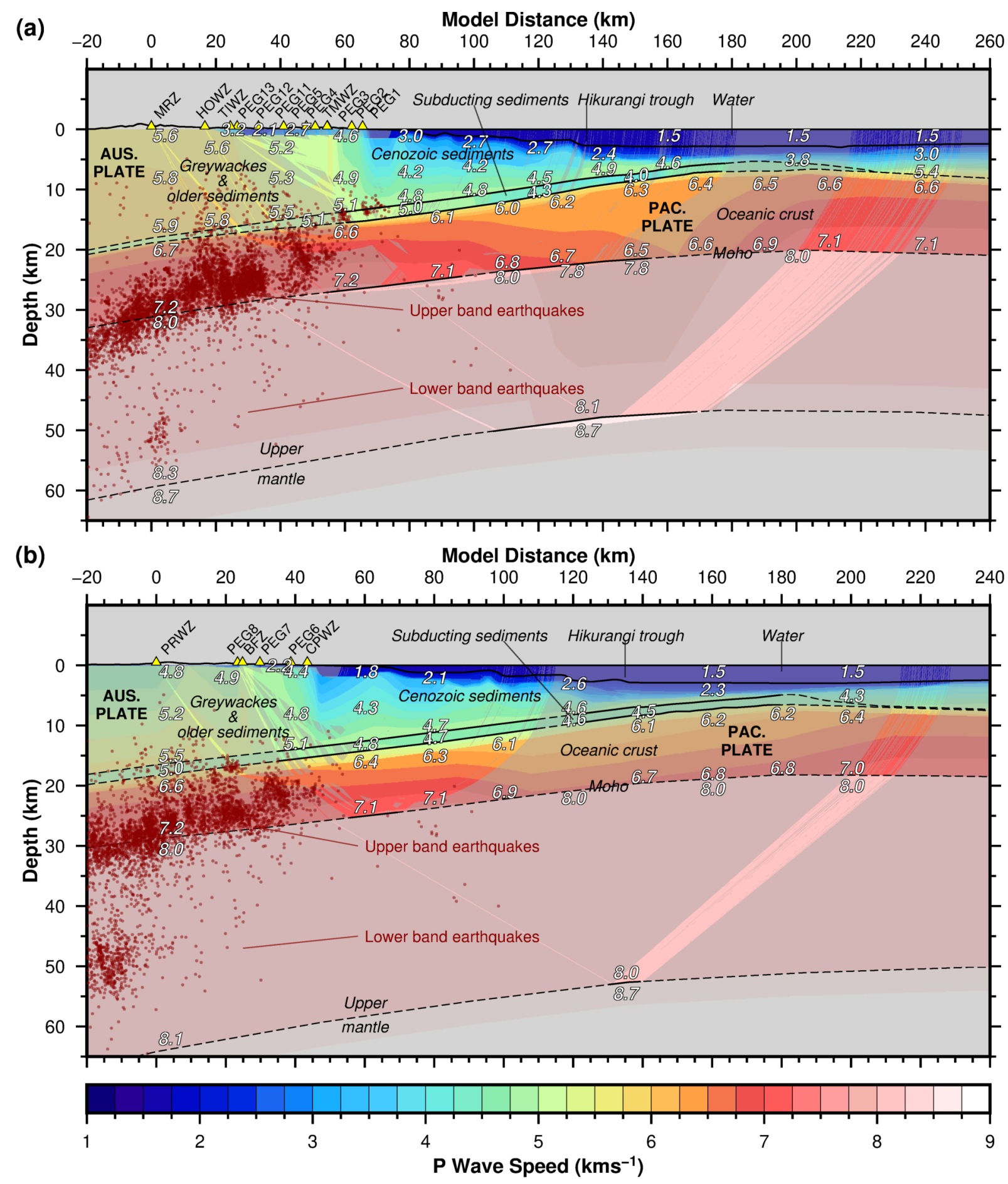

Figure 3.6 Final velocity models along (a) PEGASUS23 transect and (b) PEGASUS25 transect. Note that in (a), station symbols for TMWZ and PEG3 cannot be distinguished as they are very close to each other. Continuous black lines are confirmed layer boundaries. Dashed black lines are inferred layer boundaries. Grey shading indicates areas in the velocity model without ray coverage. White numbers with black outline are P-wave-speeds at those locations in $\mathrm{km} / \mathrm{s}$. Red dots indicate the perpendicularly projected earthquake hypocentres within $50 \mathrm{~km}$ from each transect. Hypocentre relocations are from Eberhart-Phillips et al. [2017]; Evanzia et al. [2018]; Reyners et al. [2011]; Zhang and Thurber [2006]. AUS. PLATE and PAC. PLATE are Australian Plate and Pacific Plate respectively. 

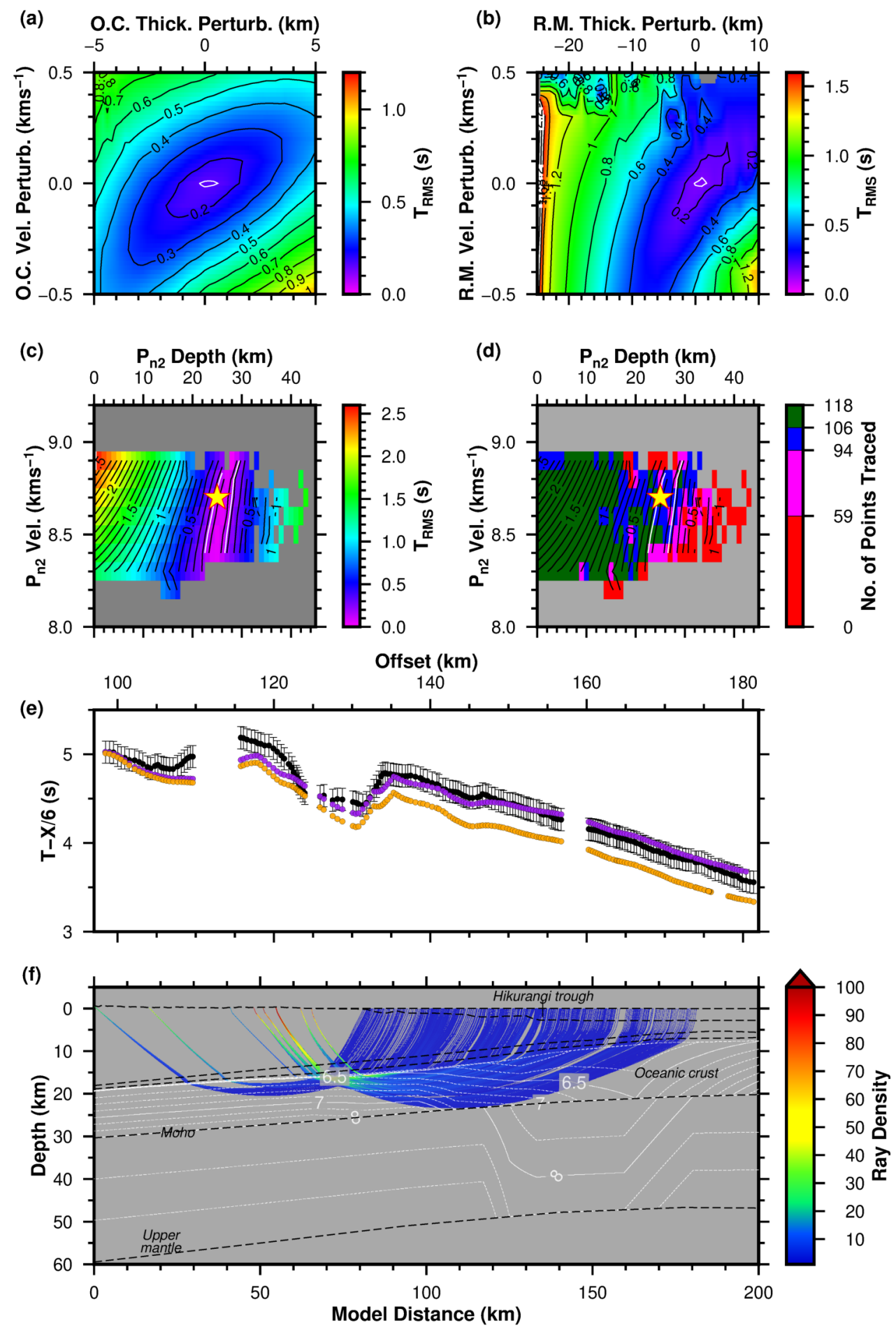

Figure 3.7 (a) Perturbation test to estimate the uncertainties in the determined thickness and velocities within the oceanic crust (O.C.). (b) Perturbation test to estimate the uncertainties in the determined thickness and velocities within the regular mantle (R.M.). (c) Grid-search test to estimate the uncertainties in the determined depth from the Moho and velocity of the faster upper mantle layer $\left(\mathrm{P}_{\mathrm{n} 2}\right)$. (d) Number of travel-time picks traced for depth-velocity pairs in (c). White contour in (a)-(d) indicates the $95 \%$ confidence interval. Yellow stars with red outline indicate the preferred values for velocity and depth in (c) and (d). (e) Sensitivity test to assess the robustness of the velocity determination of the crust with offset using the refracted phase $\left(\mathrm{P}_{\mathrm{o}}\right)$ in the oceanic crust at PEG3 station with the shots of PEGASUS23 line. Black lines with error bars are the observed travel-times. Purple dots are calculated travel-times with the P-wave-speed reduction in the oceanic crust as in the preferred velocity model. Orange dots are calculated travel-times without the P-wave-speed reduction in the oceanic crust. (f) Plot of velocity contours (white) indicating the drop in P-wave-speed resolved in the crust and the upper mantle of Hikurangi Plateau beneath the Hikurangi trough along PEGASUS23 transect. Contour interval is $0.1 \mathrm{~km} / \mathrm{s}$. This is superimposed on density of rays turning exclusively in the Hikurangi Plateau crust. 
(Figure A.30). In the PEGASUS25 transect, it is the top of the oceanic crust that aligns well with the plate interface of Williams et al. [2013] (Figure A.31). This is within the depth accuracy of $2 \mathrm{~km}$ suggested by Williams et al. [2013] for the hypocentral depths, which were primarily used to define the plate interface beneath the North Island.

\subsubsection{Crustal Structure of Subducting Pacific Plate}

The layer of subducting sediments (Figure 3.6) is a poorly resolved feature in the velocity models presented here. Its thickness in the offshore region with MCS coverage is $\sim 2 \mathrm{~km}$ (Figures A.21-A.23). In the refraction data, there is no direct evidence for its existence under the onshore regions, because a low-velocity layer is incapable of producing first arrivals. However, towards the south of the two transects, along the onshore portion of the SAHKE01 transect beneath the North Island, its thickness is consistently found from reflection data to be $\sim 2 \mathrm{~km}$ [Henrys et al., 2013; Tozer et al., 2017]. We therefore model the subducting sediments as a $\sim 2 \mathrm{~km}$ thick layer $\left(\mathrm{V}_{\mathrm{P}} \sim 4.5-5 \mathrm{~km} / \mathrm{s}\right)$, with some variation in the PEGASUS23 transect towards the northwestern end (0-40 km PEGASUS23 transect model distances) to fit the travel-times.

The velocity structure of the HP crust is modelled as two layers, a $\sim 2 \mathrm{~km}$-thick layer with a steep velocity gradient $\left(\mathrm{V}_{\mathrm{P}} \sim 5.5-6.2 \mathrm{~km} / \mathrm{s}\right)$ underlain by a $\sim 10 \mathrm{~km}$-thick layer with a gentle velocity gradient. Thus, the combined thickness of the HP crust is $12 \pm 1$ $\mathrm{km}$ throughout (Figures 3.6, 3.7a and Table 3.2). The highest $\mathrm{V}_{\mathrm{P}}$ resolved at the base of the HP crust is estimated to be $\sim 7.1 \pm 0.1 \mathrm{~km} / \mathrm{s}$, based on long-offset refracted phases in the crust $\left(\mathrm{P}_{\mathrm{o}}\right)$ and reflections from the Moho $\left(\mathrm{P}_{\mathrm{m} 1} \mathrm{P}\right)$. This is similar to the highest $\mathrm{V}_{\mathrm{P}}$ of $7.25 \pm 0.35 \mathrm{~km} / \mathrm{s}$ reported for the HP crust along SAHKE01 line [Mochizuki et al., 2019]. P-wave-speed along the upper boundary of the $\sim 10 \mathrm{~km}$ thick layer of the crust for both transects is generally $\sim 6.6 \pm 0.1 \mathrm{~km} / \mathrm{s}$ but reduces to $\sim 6.2 \pm 0.1 \mathrm{~km} / \mathrm{s}$ beneath the central part of the transects $(90-180 \mathrm{~km}$ model distances along PEGASUS23 transect) in the vicinity of the Hikurangi trough (Figure 3.6a). Similarly, $V_{P}$ of $7.1 \pm 0.1 \mathrm{~km} / \mathrm{s}$ at the base of the crust reduces to $\sim 6.6 \pm 0.1 \mathrm{~km} / \mathrm{s}$ in the same region. Refracted waves in the crust sample much of the low-velocity zone beneath the trough (Figure 3.7f). The low-velocity zone in the crust is resolved better in the PEGASUS23 compared to the PEGASUS25 transect, as the rays in the former traverse across much of the trough region (Figure A.28 versus A.29). 
Perturbation test results indicate uncertainties of $\pm 0.05 \mathrm{~km} / \mathrm{s}$ for $V_{P}$ within, and \pm 0.5 $\mathrm{km}$ for the thickness of the crust at the $95 \%$ confidence interval (Figure 3.7a). However, conservatively we suggest uncertainties of $\pm 0.1 \mathrm{~km} / \mathrm{s}$ for $\mathrm{V}_{\mathrm{P}}$ and $\pm 1 \mathrm{~km}$ for thickness.

\subsubsection{Upper Mantle Structure of Subducting Pacific Plate}

We identify two upper mantle layers with distinct P-wave-speeds (Figure 3.6); (1) in the top $25 \mathrm{~km}$ of the mantle, a regular upper mantle with a $\mathrm{V}_{\mathrm{P}}$ of $\sim 7.8-8.2 \pm 0.2 \mathrm{~km} / \mathrm{s}$ and (2) a faster and deeper upper mantle layer with a $V_{P}$ of $\sim 8.7 \pm 0.2 \mathrm{~km} / \mathrm{s}$. However, using refracted arrivals in ocean bottom seismometers along the SAHKE01 line (Figure $3.1 b$ ) about $\sim 30 \mathrm{~km}$ south of the PEGASUS23 transect, Mochizuki et al. [2019] report a $V_{P}$ of $8.30 \pm 0.25 \mathrm{~km} / \mathrm{s}$ in the upper mantle beneath the Moho (Figure A.1).

The upper mantle layer is $25 \pm 2 \mathrm{~km}$ thick and indicates regular mantle P-wave-speeds of $8.0 \pm 0.2 \mathrm{~km} / \mathrm{s}$ (Figure 3.6). In the PEGASUS23 transect, similar to the reduction in wave-speeds in the oceanic crust beneath the trough, the mantle here $(\sim 120-180 \mathrm{~km}$ model distances) also requires a reduction in $V_{P}$ down to $7.8 \pm 0.2 \mathrm{~km} / \mathrm{s}$ (Figure 3.6a) to better fit and explain all the travel-time picks of $P_{n 1}$ phase. This reduction in $V_{P}$ is not well resolved due to lower ray coverage further to the east from $P_{n 1}$ phase. The maximum permissible $V_{P}$ at the base of this $\sim 25 \mathrm{~km}$ thick layer is $8.2 \pm 0.2 \mathrm{~km} / \mathrm{s}$ (Figure A.25). Perturbation test results indicate uncertainties of $\pm 0.05 \mathrm{~km} / \mathrm{s}$ for $V_{P}$ within and $\pm 0.5 \mathrm{~km}$ for the thickness of the regular mantle at the $95 \%$ confidence interval (Figure $3.7 \mathrm{~b})$. However, we suggest conservative uncertainties of $\pm 0.2 \mathrm{~km} / \mathrm{s}$ for $\mathrm{V}_{\mathrm{P}}$ and $\pm 2 \mathrm{~km}$ for the thickness.

P-wave-speeds of $8.7 \pm 0.2 \mathrm{~km} / \mathrm{s}$ in the faster, deeper upper mantle layer were recorded as first arrivals on three gathers with lengths of $\sim 35 \mathrm{~km}, \sim 15 \mathrm{~km}$ and $\sim 20 \mathrm{~km}\left(\mathrm{P}_{\mathrm{n} 2}\right.$ phase in Figures 3.4a, 3.5a and A.20a). The depth to the faster upper mantle layer is $\sim 50 \mathrm{~km}$ from the surface (25 $\pm 2 \mathrm{~km}$ from the Moho) (Figures 3.6, A.24c, $\mathrm{d}$ and A.25). The rays sample a maximum lateral section of $\sim 70 \mathrm{~km}$ of the refracting boundary of the fast, upper mantle under the Hikurangi trough. This boundary is confirmed only between 105-175 km model distances along PEGASUS23 transect (Figure 3.6a). This refracting boundary has been extended parallel to the Moho towards either ends of the two transects for simplicity. According to the grid-search test (Figure 3.7c, d), there is a trade-off of $\pm 2 \mathrm{~km}$ with the depth to the layer, and the speed is determined to $\pm 0.2 \mathrm{~km} / \mathrm{s}$. The depth to the Moho under the western Chatham Rise in the region 
east of the Chatham Islands is estimated to be $\sim 25 \mathrm{~km}$ with a $\sim 12 \mathrm{~km} \mathrm{HP}$ crust [Grobys et al., 2008; Riefstahl et al., 2020]. Taking this into account, the Moho depth at model distances $>150 \mathrm{~km}$ (Figure 3.4c) is very likely to follow the unconfirmed Moho in the model. A jump in Moho depth could be expected at about $200 \mathrm{~km}$ from the 150 $\mathrm{km}$ model distance $(350 \mathrm{~km}$ model distance if the model were to be extended eastwards), where the sediments of the Chatham Rise are thicker. Therefore, the effect of the unresolved Moho in the determination of the depth to this faster upper mantle layer is not significant. Furthermore, to assess the effect of the velocity structure of the $\mathrm{HP}$ crust at model distances $>150 \mathrm{~km}$, in the determination of the depths to the deeper upper mantle layers, delay times between the final preferred model and after applying velocities of $6.60-7.20 \mathrm{~km} / \mathrm{s}$ for the base of the HP crust at model distances $>150 \mathrm{~km}$ for $\mathrm{P}_{\mathrm{n} 2}, \mathrm{R}_{1}$ and $\mathrm{R}_{2}$ phases were calculated (Figure A.32). The maximum delay times of $\sim 0.13 \mathrm{sec}$ are within the pick uncertainties of these phases (Table 3.1). Therefore, the velocity structure of $\mathrm{HP}$ at model distances $>150 \mathrm{~km}$ has negligible effect on the determination of the depths of the deeper upper mantle layers.

The interfaces from which $R_{0}, R_{1}$ and $R_{2}$ reflections occur can be placed at depths of $\sim 65-80 \mathrm{~km}$ in the mantle from raytracing (Figures 3.4c, 3.5c and A.14c).

\subsection{Discussion}

\subsubsection{Oceanic Crustal Thickness of Hikurangi Plateau}

Well-constrained refracted and reflected phases indicate that the Hikurangi Plateau crust (including the $\sim 2 \mathrm{~km}$ thick overlying low $\mathrm{V}_{\mathrm{P}}$ volcanoclastic layer) is $12 \pm 1 \mathrm{~km}$ thick. This is consistent with previous inversions of earthquake data [Bannister, 1988; Brisbourne and Stuart, 1998; Galea, 1992; Savage et al., 2007] and controlled-source studies from the SAHKE transect [Henrys et al., 2013; Mochizuki et al., 2019; Tozer et al., 2017]. We suggest that the very large estimate of $35 \mathrm{~km}$ [Reyners, 2012] is because the double seismic zone was used to represent the subducting crust. Nevertheless, even a $12 \mathrm{~km}$ thickness is $\sim 70 \%$ greater than the regular oceanic crustal thickness of $\sim 7 \mathrm{~km}$ [White et al., 1992] and thus represents a significant buoyancy anomaly [Mochizuki et al., 2019]. At $12 \mathrm{~km}$ thick, the crust is only one third of the maximum crustal thickness of the Ontong-Java Plateau [Coffin and Eldholm, 1994], but it is consistent with the western margin of the Manihiki Plateau [Hochmuth et al., 2019], which also was a part of the original much larger Ontong-Java-Manihiki-Hikurangi Plateau 
[Taylor, 2006]. The lower buoyancy induced by the thinner crust of the HP compared to that of the thicker crust of Ontong-Java Plateau explains why the former readily subducts and the latter resists subduction.

Oceanic plateaus have thicker crust than regular oceanic crust. Most of them are considerably thicker than the HP. Kerguelen Plateau crust in the southern Indian ocean is $\sim 17 \mathrm{~km}$ thick [Charvis et al., 1995]. Ninety East Ridge crust has a maximum thickness of $\sim 24 \mathrm{~km}$ [Grevemeyer et al., 2001]. Caribbean Plateau crust is 8-15 km thick [Kerr, 2014]. Rio Grande Rise off eastern South America has a maximum crustal thickness of $\sim 25 \mathrm{~km}$ [Garcia et al., 2019]. Thus, when compared with most other oceanic plateaus, the HP crust is thinner. Such variations in crustal thickness of this order are possible for an oceanic plateau because crustal thickness is a function of mantle temperature and spreading rate [e.g. White et al., 1992].

\subsubsection{High Upper Mantle P-wave-speeds of Hikurangi Plateau}

P-wave-speeds of $8.5-9.0 \mathrm{~km} / \mathrm{s}$ are documented at sub-Moho depths within the HP just east of and beneath the North Island (Figure A.1). These high speeds were first detected 35 years ago along a northeast-southwest azimuth from earthquake travel-times [Kayal and Smith, 1984], and the prevailing interpretation was that the high speeds result from anisotropy created by flow-induced orientation of mantle olivine crystals [Brisbourne and Stuart, 1998; Chadwick, 1997; Galea, 1993]. Chadwick [1997] also reports a high-speed mantle of $V_{P}=8.7 \mathrm{~km} / \mathrm{s}$ beneath a regular mantle of $\mathrm{V}_{\mathrm{P}}=8.2 \mathrm{~km} / \mathrm{s}$ under the eastern North Island. In Chadwick's [1997] profile (Figure A.1), the high-speed layer is $\sim 8-10 \mathrm{~km}$ below the Moho beneath the eastern North Island (Figure A.33). Similar wave-speeds within the upper mantle measured along two northwest-southeast azimuths are also obtained from a well-located earthquake recorded by the $\sim 900$ channel onshore array of SAHKE [Stern et al., 2018]. In addition, Chong [1982]; Eberhart-Phillips et al. [2010]; Robinson [1986] reveal P-wave-speeds of 8.6-9.0 km/s for the upper mantle in margin-oblique azimuths (Figure A.1). Thus, the higher wave-speeds in the upper mantle of the HP are well documented.

The presence of similar wave-speeds in mutually perpendicular directions requires revision to the previous interpretation of an azimuthally anisotropic mantle. One interpretation is that the high speeds are due to the presence of eclogite [Love et al., 2015]. However, the shallowest depth at which we observe these high speeds is $\sim 32$ 
km along Chadwick's [1997] profile (Figure A.1), and eclogite only becomes stable at depths $>44 \mathrm{~km}$ and temperatures $>500^{\circ} \mathrm{C}$ [Hacker, 1996]. Moreover, the wave-speeds are too high for eclogite $(7.0-8.2 \mathrm{~km} / \mathrm{s})$ [Kobussen et al., 2006]. An alternative explanation is that the high wave-speeds are due to a distinct fabric with radial anisotropy developed in a plume setting [Stern et al., 2020]. This fabric has a low wave-speed in the vertical direction and can therefore be described as radially anisotropic, yet azimuthally isotropic.

\subsubsection{Hydration and Dehydration of the Subducting Slab}

P-wave-speeds in the HP crust and upper mantle show a reduction beneath the trough (Figures 3.6, 3.7e, f), where plate bending-induced normal faults occur. Similar reductions in crustal and upper mantle P-wave-speeds have been reported for most subduction zones (e.g. Middle America [Grevemeyer et al., 2007]; Van Avendonk et al. [2011]; North Chile [Ranero and Sallarès, 2004]; Alaska [Shillington et al., 2015]; Kermadec [Bassett et al., 2016]; Northern Japan [Fujie et al., 2018]).

Bending-induced normal faults provide fluid migration pathways and can result in hydration of the subducting slab. For water to infiltrate into the slab, strong negative pressure gradients are required to overcome the lithostatic pressure. From numerical modelling, Faccenda et al. [2009] suggest that sub-hydrostatic or even negative pressure gradients could exist, which result in downward pumping of fluids along the normal faults into the upper mantle. However, Korenaga [2017] argues that the magnitudes of such pressure gradients are insufficient to overcome the confining pressure and suggests that development of crack porosity by thermal cracking, further enhanced by bending-related faulting is enough to explain the bending-related velocity anomalies in the subducting crust and upper mantle. On the other hand, Naif et al. [2015] show highly conductive normal faults within the subducting crust at the middle America trench, suggesting percolation of water into the crust. Furthermore, Miller and Lizarralde [2016] suggest that serpentinised normal faults within the upper mantle are capable, on their own, of reducing wave propagation speed from the background mantle wave-speeds.

The presence of normal faults in the oceanic crust is evident from seismic reflection images along SAHKE01 line [Bland et al., 2015]. Further evidence for normal faults within the subducting oceanic crust comes from seismic reflection data, which 
indicate that reactivated normal faults facilitate kinking of the subducting oceanic crust under the central North Island [Henrys et al., 2006]. Du et al. [2004] also correlate northeast-striking and westerly dipping linear seismic features from a relocated earthquake catalogue in the Wellington region with normal faults within the subducting Pacific Plate. Although these normal faults lie beneath the North Island, westward of the Hikurangi trough, they are likely to have originated by bending of the slab in the outer rise region. Furthermore, a recent relocated earthquake catalogue in the region shows that the subducting slab in the eastern part of the southern North Island is under extension oblique to the margin, resulting in normal faults, and under compression parallel to the margin, resulting in strike-slip faults [Evanzia et al., 2017]. A radius of curvature of $149 \mathrm{~km}$ for the subducting slab under the southern North Island [Evanzia et al., 2018], results in a 10\% increase in length (extension) of the subducting slab due to bending, causing normal faulting. Normal faults that originate at the top of the oceanic crust by bending can potentially extend as deep as $25 \mathrm{~km}$ into the upper mantle of the subducting slab [Emry and Wiens, 2015; Faccenda et al., 2009] and nearly up to half of the plate thickness [Garcia et al., 2019].

Previous seismic studies that detected the high $V_{P}(>8.5 \mathrm{~km} / \mathrm{s})$ upper mantle layer under the North Island (Figure A.1) also found that it started at depths down to 15 km below the Moho [Brisbourne and Stuart, 1998; Chadwick, 1997; Galea, 1992; Robinson, 1986]. We interpret the increase in thickness of the lower wave-speed $(\sim 8 \mathrm{~km} / \mathrm{s})$ mantle beneath the trough to reflect the presence of a down-warped low-velocity zone, similar to the zone of reduced low-velocity zone in the crust. The $\sim 10 \%$ reduction in $V_{P}$ in both crust and upper mantle observed here can be explained in two ways. First, it could result from the hydration of the crust and the upper mantle through bending-induced normal faults. This leads to the formation of hydrous minerals in the crust (e.g. hornblende, chlorite) and in the mantle (e.g. antigorite). A $\sim 10 \%$ reduction in $\mathrm{V}_{\mathrm{P}}$ would result from $\sim 40 \mathrm{wt} \%$ alteration of the gabbroic crust ( $~ 0.5 \mathrm{wt} \%$ water) and $\sim 20 \mathrm{wt} \%$ serpentinisation of mantle peridotite ( $\sim 3 \mathrm{wt} \%$ water) [Korenaga, 2017]. This is the most widely reported explanation for similar observations at other subduction zones [e.g. Ranero and Sallarès, 2004; Shillington et al., 2015; Van Avendonk et al., 2011]. Thus, the $\sim 25 \mathrm{~km}$ thick "regular mantle" ( $\mathrm{V}_{\mathrm{P}} \sim 8$ $\mathrm{km} / \mathrm{s}$ ) here is not in fact regular, but rather the high $V_{P}$ mantle has mechanically bent, deformed and hydrated. Further evidence for deformation of the low-velocity zone is 
the observation of a "shadow zone" between $\mathrm{P}_{\mathrm{n} 1}$ and $\mathrm{P}_{\mathrm{n} 2}$ phases in Figures 3.4a (140-220 km offsets) and A.20a (100-160 km offsets), implying attenuation due to cracking and/or water. Therefore, we do not see any arrivals at these offsets. Alternatively, the $\sim 10 \%$ reduction in $\mathrm{V}_{\mathrm{P}}$ could be due to the development of crack porosity by thermal cracking [Korenaga, 2017]. For the $120 \mathrm{Ma}$ old HP, cracks as deep as 20-30 km can be expected to form with an interval of a few tens of kilometres [Korenaga, 2017]. In contrast to the tensional normal faults, if the thermal cracks are open, downward water pumping is possible and this would result in rapid serpentinisation of the thermal cracks. As the plate starts to subduct, normal faults would further enhance the porosity introduced by thermal cracking.

The upper mantle velocities of $8.30 \pm 0.25 \mathrm{~km} / \mathrm{s}$ beneath the Moho near the Hikurangi trough along the SAHKE01 transect reported by Mochizuki et al. [2019] are relatively faster than those reported in this study, but still slower than the faster upper mantle velocities $>8.5 \mathrm{~km} / \mathrm{s}$ reported in the region. This could be indicative of a lower degree of hydration due to serpentinisation and/or water filled cracks or the seismic waves sampling the fractures at a slightly different angle, along the SAHKE01 line.

Double seismic zones in the subducting slab have been identified for almost all the subduction zones in the world [Brudzinski et al., 2007]. The upper and lower bands of double seismic zones are separated vertically by an aseismic or weakly seismic zone up to $40 \mathrm{~km}$ thick [Hacker et al., 2003]. The upper band is thought to represent the seismicity that results from the dehydration of the crust and the lower band is thought to represent the seismicity that results from the dehydration of antigorite in the mantle [Brudzinski et al., 2007]. The presence of a double seismic zone beneath the southern North Island has been established by Du et al. [2004]; Eberhart-Phillips et al. [2017]; Evanzia et al. [2017]; Reyners et al. [2011]; Robinson [1986]; Zhang and Thurber [2006]. The separation between the two bands of seismicity is about $30 \mathrm{~km}$. The upper band of seismicity aligns well with the subducting crust and probably indicates the dehydration of altered gabbroic crust (Figure 3.6). The onset of the lower band of seismicity under the North Island corresponds to where high upper mantle $V_{P}$ of $8.7-9.0 \mathrm{~km} / \mathrm{s}$ is reported to be about $10-15 \mathrm{~km}$ underneath the Moho (Figure 3.8). This is about $80 \mathrm{~km}$ west of the Hikurangi trough. The serpentinised mantle, either through fluid-rock interactions along normal faults or through the increase in 
porosity first introduced by thermal cracks, dehydrates here at temperatures of $\sim 500-550^{\circ} \mathrm{C}$ [Fagereng and Ellis, 2009] and pressures of $\sim 1.0-1.2 \mathrm{GPa}$ [Guillot et al., 2015], and the low velocities now revert to the high velocities of $8.7-9.0 \mathrm{~km} / \mathrm{s}$. This phenomenon explains the variation in the depth to the faster upper mantle layer in Figure 3.8. It also provides evidence to support the hypothesis that the lower band of earthquakes in the double seismic zone is due to serpentinite dehydration processes [Brudzinski et al., 2007].

The higher buoyancy of subducting thick crust could result in shallower subduction angles and less bending-related faulting. Despite this, evidence for bending-induced faulting in the outer rise region and possible hydration within the crust and upper mantle can be found in the subducting 135 Ma old Indian Plate in eastern Java where the crustal thickness varies from 9 to $18 \mathrm{~km}$ [Planert et al., 2010; Shulgin et al., 2011]. Much younger ( 29-51 Ma old), but thick ( $\sim 15 \mathrm{~km})$, crust of the Nazca Ridge subducts at the Peruvian margin in Chile with a subduction angle $\sim 10^{\circ}$ [Contreras-Reyes et al., 2019], before being subjected to flat slab subduction further onshore. The presence of bending-induced normal faults in the subducting Nazca ridge is evident from MCS images [Hampel et al., 2004]. Yet, bending-related velocity reduction in the subducting crust or in the upper mantle is not observed [Contreras-Reyes et al., 2019]. The extent of buoyancy depends on both crustal thickness and the age of oceanic plateaus. Young plateaus are more likely to resist subduction than an older plateau of similar crustal thickness. This is because the crust becomes less buoyant as it cools [Cloos, 1993; Kerr, 2005]. Thus, although the Hikurangi Plateau crust is thick, its age ( $\sim 120 \mathrm{Ma})$ may control its ability to bend (Figure A.34), hydrate, and undergo subduction. The hydration of the subducting Hikurangi Plateau appears analogous to processes documented elsewhere in oceanic crust although spatial variations in the degree of hydration could be present along the margin.

\subsubsection{Deep-lithospheric Reflections from the Pacific Plate}

$\mathrm{R}_{0}$ reflection at $\sim 35 \mathrm{sec}$ two-way travel-time is observed only on one gather (Figure $3.5 \mathrm{a})$ and is most likely to be a reflection from the top of a much faster $\left(\mathrm{V}_{\mathrm{P}} \sim 8.9 \mathrm{~km} / \mathrm{s}\right)$ upper mantle layer at a depth of $\sim 65 \mathrm{~km}$ (Figure 3.5c). $\mathrm{R}_{1}$ and $\mathrm{R}_{2}$ reflections at $\sim 37$ $40 \mathrm{sec}$ two-way travel-time, or $\sim 70-80 \mathrm{~km}$ deep, are recorded on three receiver gathers 


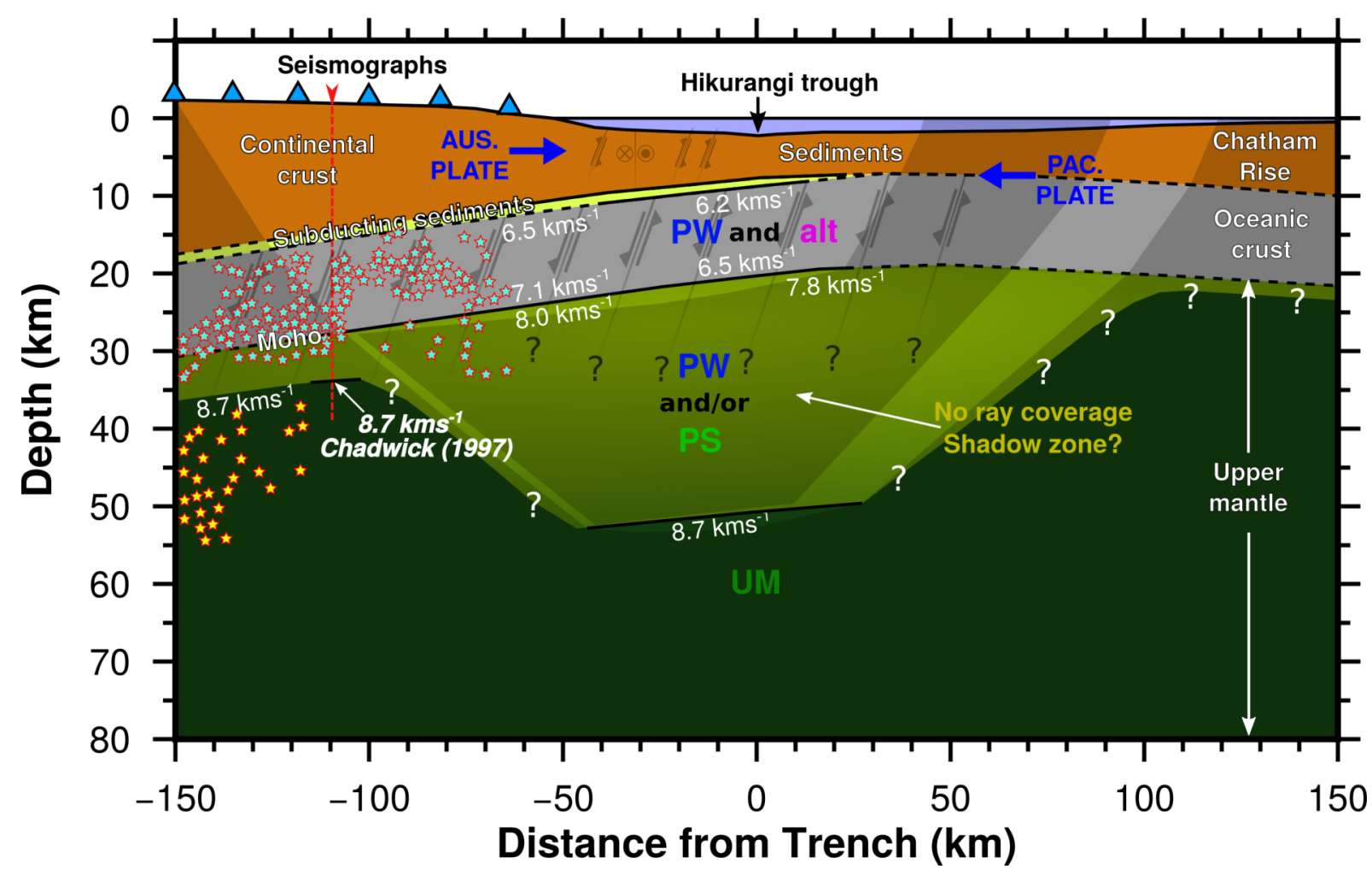

Figure 3.8 Conceptual model of the Hikurangi Plateau being subjected to bending by subduction showing the interpreted variation in depth to the high-speed upper mantle layer from the onshore region to the trough. Intersection with Chadwick's [1997] profile is denoted by the red dashed line. Also shown in red is the depth and P-wave-speed of the faster upper mantle layer of Chadwick [1997]. Light blue and yellow stars with red outline indicate the hypocentres of the upper and lower bands of the double seismic zone reported by Du et al. [2004], respectively. Black lines indicate faults. Normal and reverse faults are indicated by arrows showing relative movement on either side of the faults. Sense of movement along strike-slip faults is denoted by cross (going into the page) and dot (coming out of the page). Faults on the seabed of the forearc west of the Hikurangi trough have been approximated from Litchfield et al. [2013]. Dark shaded regions indicate areas without ray coverage. PW-pore water, alt-alteration, PSpartial serpentinisation, UM-ultramafic. AUS. PLATE and PAC. PLATE are Australian Plate and Pacific Plate respectively. Vertical exaggeration $=4$. 
(Figures 3.4, 3.5 and A.14). The interfaces from which the $\mathrm{R}_{1}$ and $\mathrm{R}_{2}$ reflections occur correspond closely to those reported by Stern et al. [2015] from the onshore portion of SAHKE transect, which were interpreted to represent a channel at the base of the tectonic plate. These reflections are discussed in detail in Chapter 4.

\subsection{Conclusions}

The conclusions of this study are as follows:

1. The crustal thickness of the Hikurangi Plateau, east of the North Island, is $12 \pm 1$ $\mathrm{km}$.

2. There is a high P-wave-speed upper mantle $(8.7 \mathrm{~km} / \mathrm{s})$ resident beneath a $\sim 25 \mathrm{~km}$ thick mantle of $\mathrm{V}_{\mathrm{P}} \sim 8 \mathrm{~km} / \mathrm{s}$ under the Hikurangi trough, and the upper mantle of this part of the Hikurangi Plateau appears to be azimuthally isotropic.

3. There is an apparent $\sim 10 \%$ drop in P-wave-speed beneath the Hikurangi trough in the subducting Hikurangi Plateau crust and upper mantle. We infer that it is a result of hydration through bending-induced normal faults and/or the enhancement of crack porosity originally formed by thermal cracking.

4. The upper and lower bands of seismicity in the double seismic zone observed in the southern Hikurangi margin are likely to be due to dehydration of the altered gabbroic crust and the mantle serpentinite, respectively.

\section{Acknowledgements}

We would like to thank the SAHKE Field Team and the crew of the $M / V$ Reflect Resolution for their efforts in collecting the data used in this study. Productive discussions with Simon Lamb helped to improve the interpretations and discussion. We are also thankful to Alexey Shulgin and an anonymous reviewer whose comments helped improve the paper published in Earth and Planetary Science Letters encompassing this study. Adrian Benson (now at GNS Science) and Aleksander Balieav (Victoria University of Wellington) are acknowledged for their technical support. 


\title{
Chapter 4
}

\section{Evidence for a Lithosphere-Asthenosphere Boundary Zone of the Pacific Plate at the Southern Hikurangi Margin}

\begin{abstract}
Controlled-source, onshore-offshore seismic profiling off eastern North Island, New Zealand, reveals a series of distinct reflection horizons from depths of 70-85 km. These wide-angle reflections are interpreted as coming from the base of the Pacific Plate as they line up with the same down-dip reflections identified from an earlier onshore study based on near vertical incidence reflections. The horizons can thus be traced as continuous over a length scale of $\sim 100 \mathrm{~km}$. From the moveout, amplitude and polarity of the reflections, we interpret the package in terms of two distinct layers: a 3-5 km thick layer with strong ( 13-15\%) azimuthal P-wave anisotropy; beneath this is a $\sim 8-10 \mathrm{~km}$ thick layer of low P-wave-speeds. This layer subdivides into an upper layer with high $\mathrm{V}_{\mathrm{P}} / \mathrm{V}_{\mathrm{S}}$ ratio $(>>2.5)$ indicating the presence of fluid (melt and/or volatiles) and a lower layer with low $\mathrm{V}_{\mathrm{P}} / \mathrm{V}_{\mathrm{S}}$ ratio $(\sim 1.85)$ with indicating a lower fluid content. Strong azimuthal anisotropy in the top layer is interpreted to be a result of olivine crystals realigning in response to the finite strain accumulated due to the differential motion between the lithosphere and asthenosphere. Our results show that it is not a single reflector or low-velocity channel that constitutes the lithosphere-asthenosphere boundary (LAB), but a series of layers that make up what we call the LAB zone (LABZ). Detection of this thin anisotropic layer would not be possible with passive-source seismic methods, and thus our results also emphasise the crucial role that controlled-source seismic methods play in defining the fine
\end{abstract}


structure at the base of a tectonic plate.

\subsection{Introduction}

Unravelling the fine structure of the lithosphere-asthenosphere boundary (LAB) has been a long-standing challenge to seismology [Fischer et al., 2010]. Up until quite recently much of the inference about the structure of the LAB has been based on passive-source seismic methods with either surface or body waves [e.g. Kawakatsu et al., 2009; Kumar et al., 2007; Rychert et al., 2010]. These data typically have frequencies less than $1 \mathrm{~Hz}$ and thus seismic wavelengths are $>10 \mathrm{~km}$. Determining fine structure at length scales less than this is difficult, and therefore the view that the $\mathrm{LAB}$ is a diffusive boundary with a length scale of $10 \mathrm{~km}$ or greater has evolved [Fischer et al., 2010]. In particular, it is viewed as a staggered zone separating convective heat-transfer below to solid state thermal conduction above the LAB. This view was in keeping with a long-standing intuition that the lithosphere is in effect a thermal boundary layer.

More recently, controlled-source seismic methods have been applied to image the LAB at different tectonic settings and a different nuance on its structure is emerging [e.g. Mehouachi and Singh, 2018; Stern et al., 2015]. These data, with frequencies as high as $14 \mathrm{~Hz}$, are near vertical reflection images from both large explosive and airgun sources, indicate a number of coherent reflective horizons that can be tracked continuously for distances of up to $200 \mathrm{~km}$ at LAB depths. Based on polarity and amplitudes, these reflections are interpreted to define a low-velocity channel in which melt has accumulated, aided by the presence of either water or carbon dioxide in the mantle. The thickness of the channel appears to reduce with increasing age of the plate [Mehouachi and Singh, 2018].

In this study, we take the investigation of the fine structure within the upper mantle one step further. We use wide-angle reflections to investigate the LAB from an onshore-offshore gather [Okaya et al., 2003] where offshore air-guns shots are recorded on static seismographs onshore. The advantage of wide-angle reflections is that enhanced amplitudes are generated when the reflections approach the critical angle (typically $>55^{\circ}$ [Castagna, 1993]). This is a key property because there are some impedance contrasts that generate significant amplitudes only at these wide angles 
and are essentially hidden from near vertical reflection surveys. This way, we identify a new reflective horizon, on top of the low-velocity LAB channel, which was not seen in previous studies. We interpret this layer as a $\sim 3-5 \mathrm{~km}$ thick azimuthally anisotropic layer generated by shear and finite strain at the base of the lithosphere.

\subsection{Structure of the Pacific Plate at Hikurangi margin}

The Pacific Plate, which contains the oceanic Hikurangi Plateau [Davy and Wood, 1994; Taylor, 2006], subducts beneath the Australian Plate at the east coast of the North Island of New Zealand (Figure 4.1a). The Pacific Plate moves with an absolute plate motion vector of $\sim 45-60 \mathrm{~mm} /$ year at an azimuth of $\sim 325^{\circ}$ [Kreemer, 2009] and a relative plate motion vector (with respect to the Australian Plate) of $\sim 45 \mathrm{~mm} /$ year at an azimuth of $\sim 260^{\circ}$ [DeMets et al., 2010] (Figure 4.1a). The depth to the base of the Pacific Plate under the southern North Island is estimated to be $\sim 73 \mathrm{~km}$ [Stern et al., 2015]. The upper 10-12 km of the plate is made of the crust of the Hikurangi Plateau as suggested from controlled-source seismic data [Henrys et al., 2013; Herath et al., 2020; Mochizuki et al., 2019; Tozer et al., 2017]. The bottom $\sim 60 \mathrm{~km}$ is made of the lithospheric mantle, which transitions into the asthenosphere through a $\sim 10 \mathrm{~km}$ thick low-velocity, sheared meltrich layer interpreted as the LAB channel [Stern et al., 2015]. A similar LAB channel is also proposed for the oceanic Atlantic Plate based on multichannel seismic reflection data [Mehouachi and Singh, 2018].

\subsection{Data}

SAHKE and PEGASUS multichannel seismic (MCS) lines of the Seismic Array Hikurangi Experiment (SAHKE) [Henrys et al., 2013] were acquired by $R / V$ Reflect Resolution in 2009/2010 (Figure 4.1b). The airgun source of the seismic vessel had a capacity of 98.3 litre $\left(6,000 \mathrm{in}^{3}\right)$ for the SAHKE lines and a shot spacing of $100 \mathrm{~m}$. These parameters were reduced to 88.5 litre $\left(5,400 \mathrm{in}^{3}\right)$ and $37.5 \mathrm{~m}$ for the PEGASUS lines. The source was towed at a depth of $6 \pm 1 \mathrm{~m}$ for both configurations. Simultaneous with the acquisition of the MCS data, the airgun sources were recorded by a network of temporary seismographs and the permanent seismographs of the GeoNet network.

The technique of using offshore airgun sources recorded at onshore seismographs is known as the "onshore-offshore" method [Okaya et al., 2003] and is visualised using 
common receiver gathers. Common receiver gathers are produced by extracting seismograms from the continuously recorded data at the seismographs using GPS-based airgun shot times and plotting them with source-receiver offset calculated using GPS-based shot locations. The common receiver gathers are further processed using a bandpass filter with $2-4-8-12 \mathrm{~Hz}$ corner frequencies and an automatic gain control to enhance the signal-to-noise ratio.

\subsection{Wide-angle Reflections in the Onshore-offshore Data}

Common receiver gathers at BFZ and MRZ stations of the GeoNet network (Figure $4.1 \mathrm{~b}$ ) were produced using the airgun shots of the SAHKE01, and at MRZ, with the airgun shots of the PEGASUS23 MCS lines. Several distinct seismic phases can be observed at two-way travel-times $>30 \mathrm{sec}$ and source-receiver offsets $>140 \mathrm{~km}$ (Figure $4.2)$.

In the common receiver gathers at BFZ and MRZ stations with the airgun shots of SAHKE01 line, we identify a band of reflectivity spanning $\sim 3.5 \mathrm{sec}$ at offsets ranging from $\sim 140-260 \mathrm{~km}$. These occur at reduced travel-times of 7-12 sec or true travel-times of about $35 \mathrm{sec}$. Within the coda of this reflectivity, several wide-angle reflections $\left(R_{0}\right.$, $\mathrm{R}_{1}, \mathrm{R}_{2}$ and $\mathrm{R}_{3}$ ) can be observed (Figure $4.2 \mathrm{a}, \mathrm{b}$ ). However, in the common receiver gather at MRZ station with the airgun shots of PEGASUS23 line, the reflection signals are weak and ( $R_{1}$ and $R_{2}$ ) can be observed (Figure 4.2c). $R_{1}$ and $R_{2}$ in this gather have lower amplitudes possibly due to the less powerful airgun source in the PEGASUS23 line. Of particular interest is the distinct relationship between $R_{0}$ and $R_{1}$ where the latter appears to onlap on to the former at offsets around 190-210 km (Figure 4.2a).

These wide-angle reflected phases arrive after the $P_{n 2}$ phase which is a refracted phase from a faster upper mantle layer with a P-wave-speed of $8.7 \pm 0.2 \mathrm{~km} / \mathrm{s}$ and at a depth of $\sim 50 \mathrm{~km}$ in the upper mantle of the Pacific Plate [Herath et al., 2020]. The parabolic nature of these late arriving phases suggest that they are wide-angle reflections rather than refractions. Furthermore, the source-receiver offsets at which they are observed confirm that they are reflections, because the shortest source-receiver offsets of these phases are less than the critical distance for a refracted arrival from a $\sim 60 \mathrm{~km}$ deep layer.

We tested the possibility of $R_{0}, R_{1}$ and $R_{2}$ being peg-leg multiples of the $P_{n 2}$ upper 


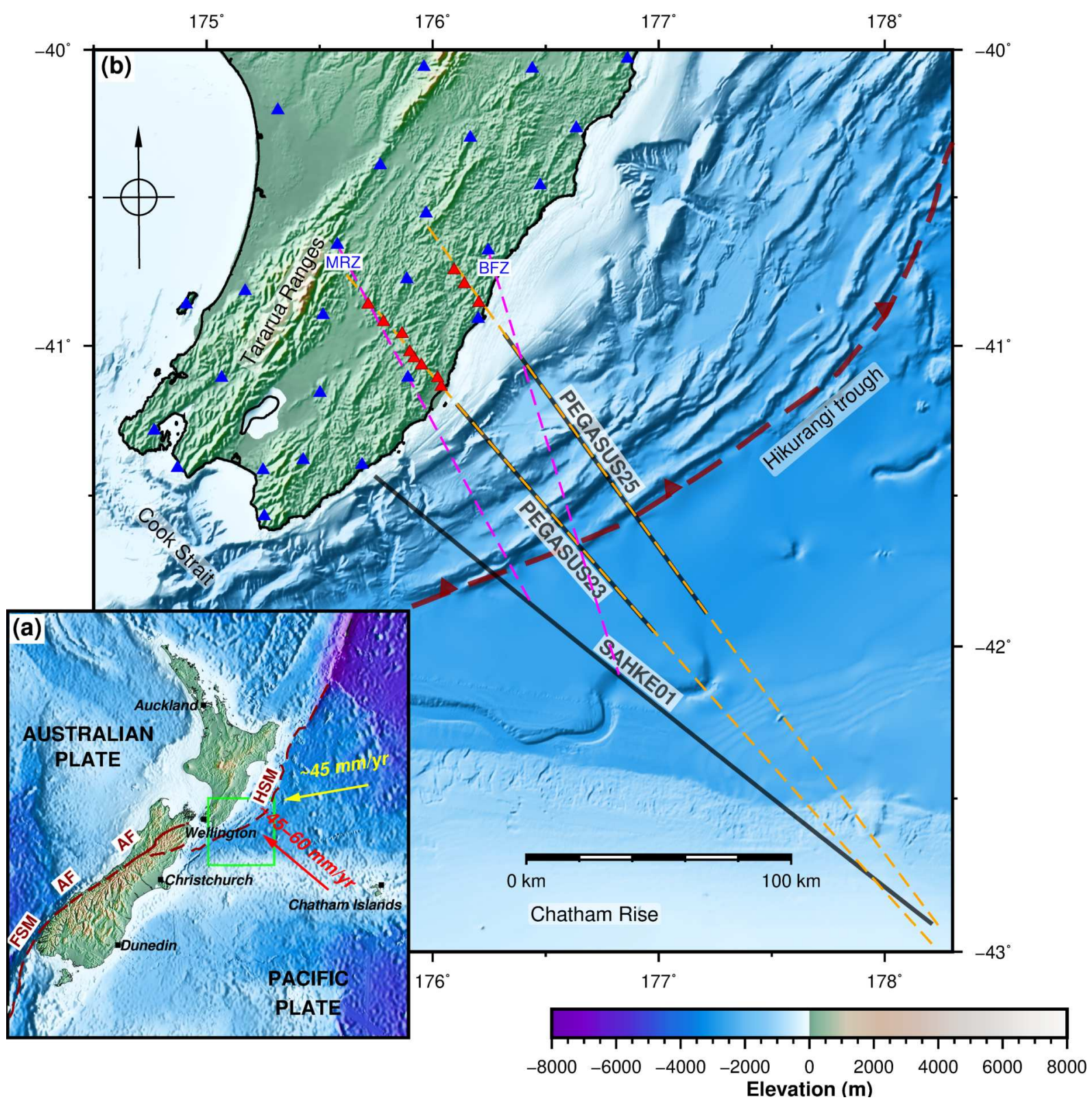

Figure 4.1 (a) Tectonic setting around New Zealand. HSM-Hikurangi subduction margin, AF-Alpine Fault, FSM-Fiordland subduction margin. Coloured vectors indicate absolute plate motion of the Pacific Plate (red) and relative plate motion of the Pacific Plate relative to Australian Plate (yellow). Green rectangle indicates the extents of (b). (b) Study area. Red triangles are temporary seismographs of the PEGASUS23 and PEGASUS25 onshore-offshore transects. Blue triangles are permanent seismographs of the GeoNet network. Black lines are offshore multi-channel seismic (MCS) lines. Orange dashed lines are the extended PEGASUS23 and PEGASUS25 onshore-offshore transects. Purple dashed lines indicate source-receiver offsets greater than $150 \mathrm{~km}$ from MRZ and $160 \mathrm{~km}$ from BFZ stations to the airgun shots of SAHKE01 line. Red dashed line marks the Hikurangi trough. 

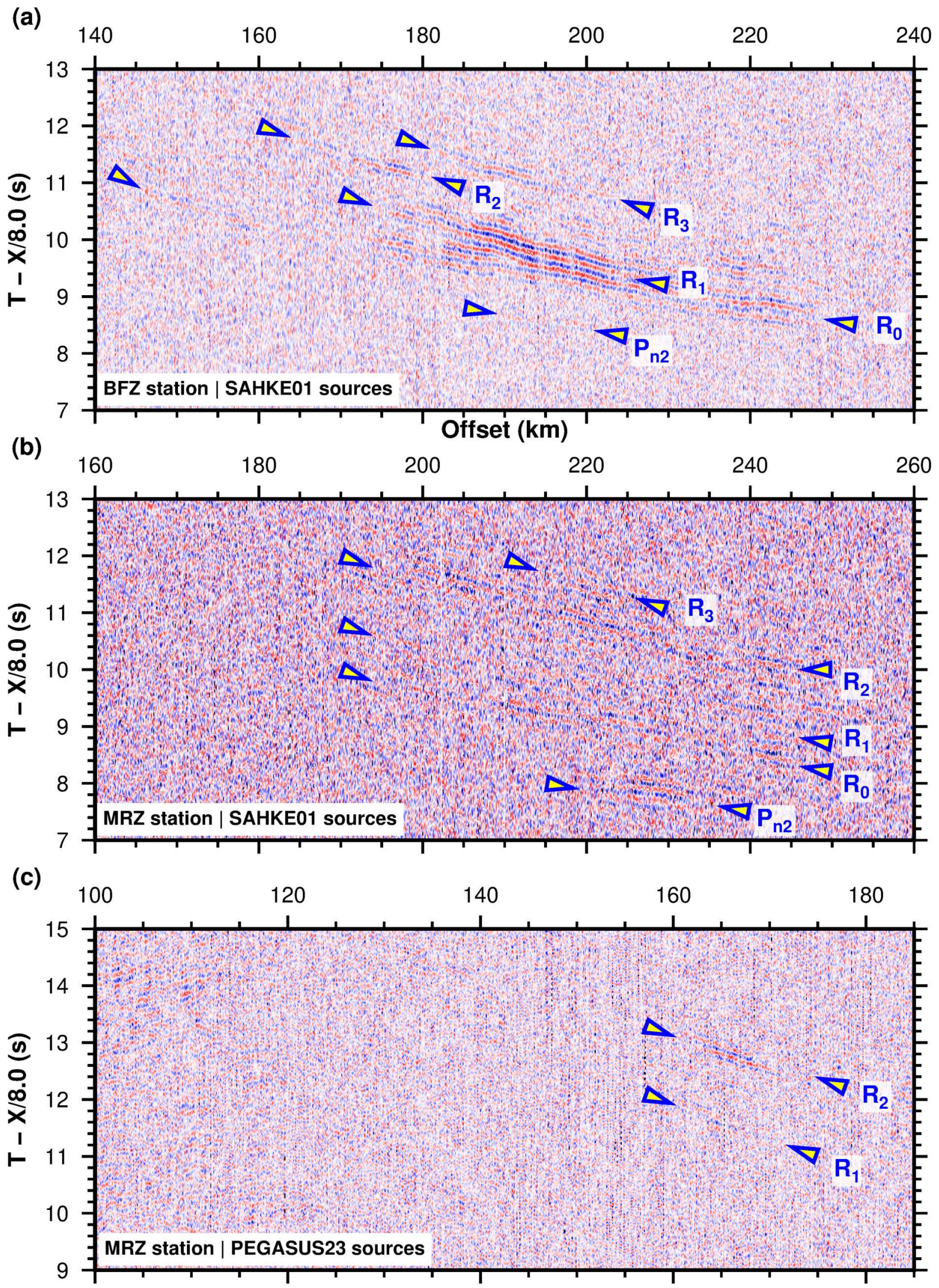

Figure 4.2 Common receiver gathers at (a) BFZ station, (b) MRZ station with airgun shots of SAHKE01 line, and (c) MRZ station with airgun shots of PEGASUS23 line. Arrows indicate the identified refractions $\left(\mathrm{P}_{\mathrm{n} 2}\right)$ and wide-angle reflections $\left(\mathrm{R}_{0}-\mathrm{R}_{3}\right)$. The gathers are processed with a bandpass filter at 2-4-8-12 $\mathrm{Hz}$ and an automatic gain control with a window of 10 secs. 
mantle refraction, within prominent reflectors in the sedimentary column. For this, the prominent reflectors in the MCS reflection data of SAHKE01 line were mapped and depth converted. Travel-time picks of $R_{0}, R_{1}$ and $R_{2}$ were raytraced using RAYINVR [Zelt and Smith, 1992] with the raypaths defined as peg-leg multiples in between reflectors A and B (Figures B.1 and B.2). The calculated travel-times could not fit the observed travel-times at the maximum offsets unless the rays bounced back and forth between the A and B reflectors up to 3 and 7 times (Figures B.1 and B.2). This is an unlikely scenario as the amplitudes decay significantly in between such reflections, and we would have expected to see earlier modes of such multiples at the same offsets further up in the record. Furthermore, the calculated travel-times could not account for the observed gradients of the phases. Secondly, we tested if these phases could be explained by a P- to S- conversion where the P-wave from the airgun source arrives at the seismograph as an S-wave after converting from an interface along its way. For this, the north and east components of the seismograms for each airgun shot were converted to radial and transverse components, and the particle motion between different components were plotted. The particle motion diagrams indicate prominent polarisation in the vertical component (Figure B.3 and B.4) and therefore the arrivals are most likely $\mathrm{P}$-waves, and not S-waves arriving at the onshore seismograph. These particle motion diagrams also suggest that these arrivals are not a result of a sideswipe (e.g. from a fault zone, vertical crack) as it would also require the particle motion to be polarised in the horizontal components.

These arrivals are recorded at two permanent seismographs of the GeoNet network, but not on the short-period temporary stations of SAHKE that were out during this time. This is proposed to be a result of the permanent broadband stations being better coupled to the ground than the temporary stations. Furthermore, the permanent seismographs, MRZ and BFZ are stationed on firm greywacke basement rocks, whereas the temporary stations were located on a variety of surface rocks. Therefore, the permanent stations have a higher chance of recording seismic energy with a high signal-to-noise ratio.

\subsection{Methodology}

We use the P-wave velocity models for the PEGASUS23 and PEGASUS25 transects of Herath et al. [2020] that constrain the P-wave velocity structure down to a depth of $\sim 50$ 
$\mathrm{km}$. In projecting the airgun shots from the SAHKE01 line onto the PEGASUS23 and PEGASUS25 velocity models (Figure 4.1), the effect from the difference in water depths at the original shot locations and the projected shot locations on the travel-times of the phases is considered negligible as the maximum travel-time delay induced is within the pick uncertainties of $0.2 \mathrm{sec}$ (Figures B.5 , B.6 and Table B.1). Using RAYINVR [Zelt and Smith, 1992], the travel-times of the wide-angle reflected phases were forward modelled in a top-down approach to minimise the travel-time residuals (Figures 4.3, B.7 and B.8 and Table B.1).

To verify the results from raytracing and to obtain an understanding of the amplitudes, we conducted seismic wave propagation simulations based upon the $2 \mathrm{D}$ velocity models obtained from raytracing. Simulations were done using SPECFEM2D, which uses a spectral element modelling approach to solve the wave-equation [Tromp et al., 2008]. SPECFEM2D was favoured over other finite-difference modelling codes as it can have a topographic free surface and coupled acoustic and elastic materials in the models, ideal for simulating wave propagation in an onshore-offshore experiment. Furthermore, the ability to include anisotropic material defined by the elastic parameters of the stiffness tensor was deemed an advantage as the lithospheric mantle consists of anisotropic minerals, predominantly olivine. The stiffness tensor for pure forsterite olivine $\left(\mathrm{Mg}_{2} \mathrm{SiO}_{4}\right)$ at $2.5 \mathrm{GPa}$, corresponding to a depth of 70-80 km in the lithospheric mantle was obtained by the linear interpolation of the elastic tensor coefficients reported by Zha et al. [1996]. Using this, the stiffness tensor for an aggregate of forsterite crystals with their crystallographic axes oriented in different directions were obtained from MTEX Matlab toolbox [Mainprice et al., 2011]. For isotropic layers, shear-wave velocities using appropriate $V_{\mathrm{P}} / \mathrm{V}_{\mathrm{S}}$ ratios between 1.8 and 2.5 and densities were used (Figure 4.4a). Attenuation was not considered in the simulations as constraints on the attenuation parameters for the offshore regions were not available. Therefore, the amplitudes of the synthetic waveforms may have been affected.

Instead of simulating a number of marine sources being recorded by a seismograph onshore, the simulations were made computationally efficient by replacing the onshore receiver by a point source and marine airgun sources by a linear array of receivers in water as reciprocity is valid for synthetic seismogram modelling [Igel, 

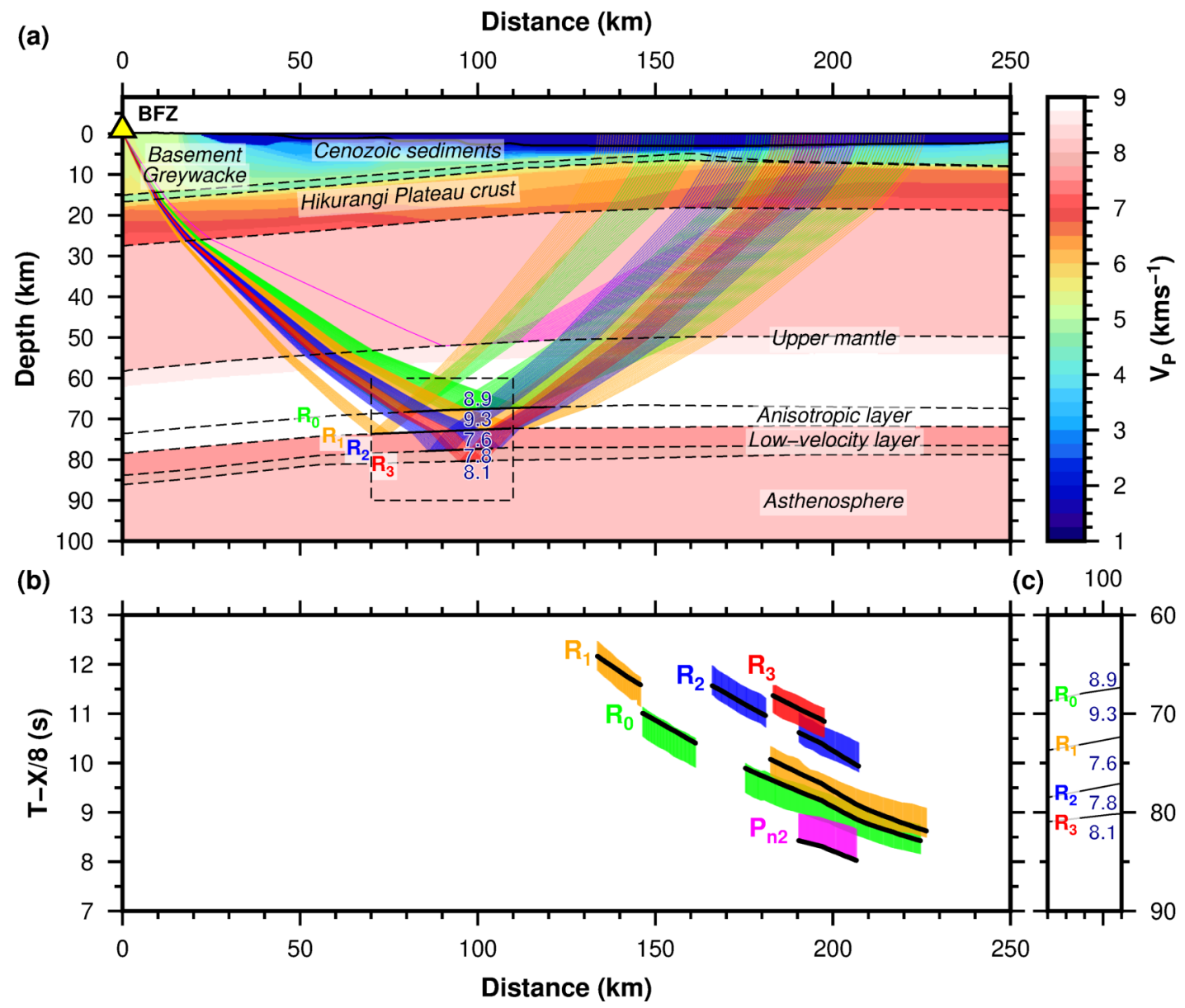

Figure 4.3 (a) Raypath diagram for the traced rays for the observed wide-angle reflections and refractions in Figure 4.2a. (b) Travel-time picks of the phases observed in Figure 4.2a colour-coded as in (a). Height of an observed travel-time pick is twice the pick uncertainty. Calculated travel-times are in black. Note that the travel-time axis is reduced with a velocity of $8.0 \mathrm{~km} / \mathrm{s}$. (c) Zoomed-up section of the region shown by the black, dashed rectangle in (a). 


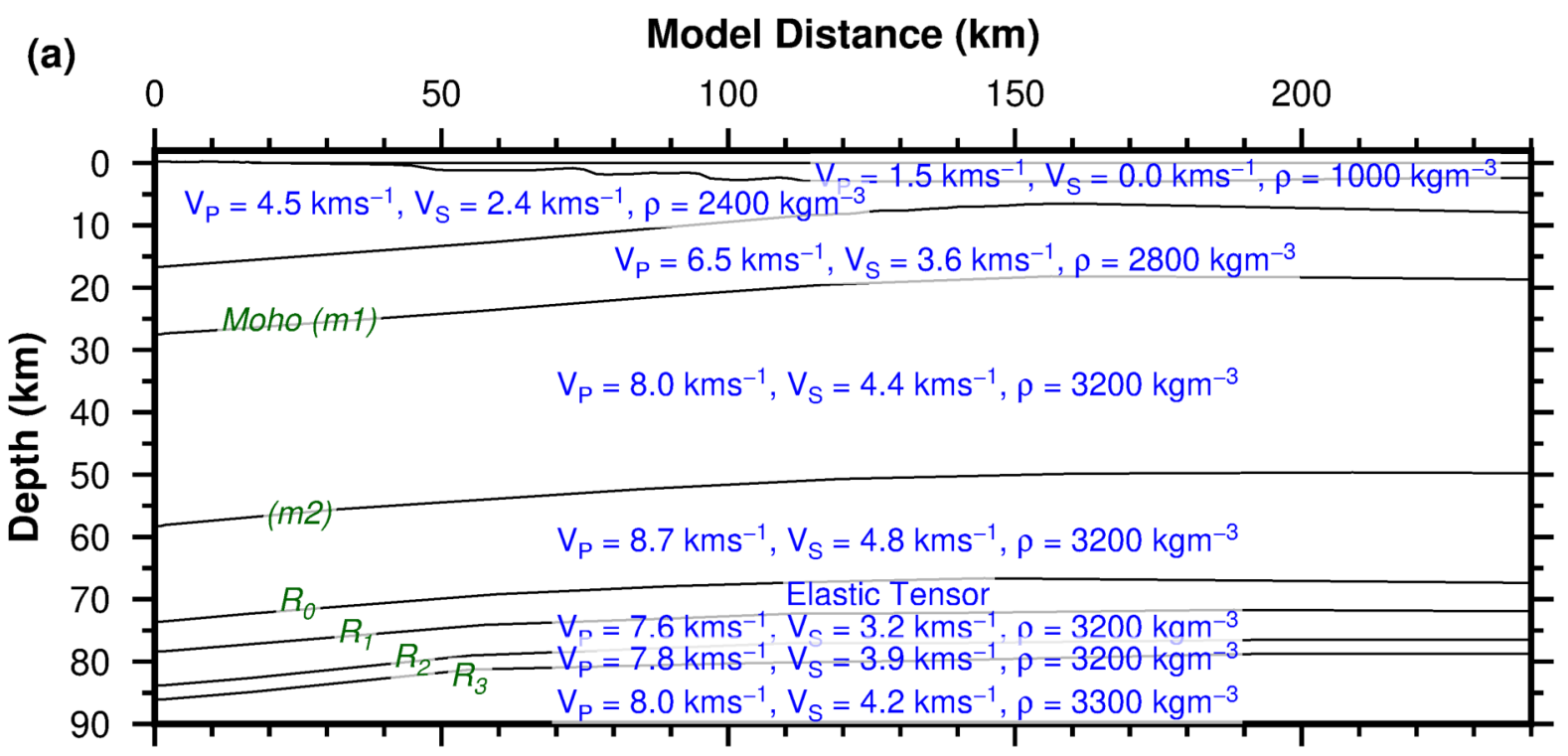

(b)

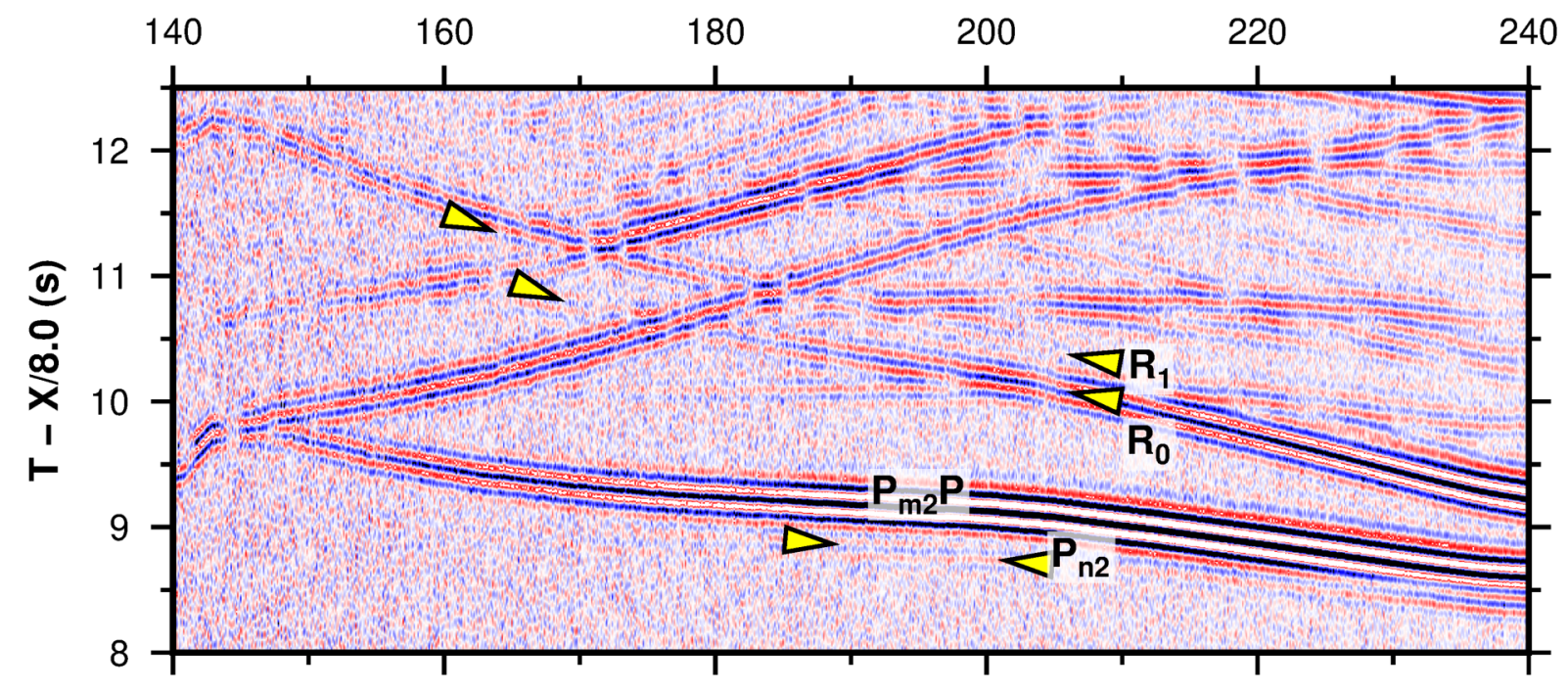

Figure 4.4 (a) Velocity and density model used to calculate synthetic common receiver gather at BFZ station with SAHKE01 airgun shots (b). The elastic tensor is given in Equation B.2. 
2017]. Thus, synthetic common receiver gathers were produced by plotting the seismograms at the source against source-receiver offset (Figure $4.4 \mathrm{~b}$ ). We used a point source for the simulation and convolved the resulting synthetic seismograms with the source-time function of the airgun array of the seismic vessel [Djeffal et al., 2021]. We also added a $1 \%$ random noise to the synthetic seismograms (Figure $4.4 \mathrm{~b}$ ).

\subsection{Depths of Reflectors and Interval Velocities}

Previous studies of the Hikurangi margin provide evidence for unusually high P-wavespeeds in the upper mantle. Tomographic inversions [Eberhart-Phillips et al., 2020] show P-wave-speeds of $9 \mathrm{~km} / \mathrm{s}$ below about $45 \mathrm{~km}$, and controlled-source work by Chadwick [1997]; Herath et al. [2020]; Stern et al. [2020] estimate P-wave-speeds of $8.7 \mathrm{~km} / \mathrm{s}$ at depths ranging from 30 to $60 \mathrm{~km}$.

In this study we adopt a P-wave speed of $8.7 \mathrm{~km} / \mathrm{s}$ at a depth of $60 \mathrm{~km}$ which increases to $8.9 \mathrm{~km} / \mathrm{s}$ just above $\mathrm{R}_{0}$ reflector at a depth of $\sim 70 \pm 2 \mathrm{~km}$ (Figures $4.3 \mathrm{a}, \mathrm{B} .7 \mathrm{a}, \mathrm{B} .8 \mathrm{a}$ and B.9). This velocity gradient is required for the $P_{n 2}$ refraction to occur. Evidence for P-wave speeds of $\sim 9 \mathrm{~km} / \mathrm{s}$ are reported in the upper mantle of the Hikurangi Plateau at depths of $\sim 60-80 \mathrm{~km}$ in the upper mantle [Eberhart-Phillips et al., 2010]. An alternative model which can account for the travel-times with slight changes in depths of the interfaces (and layer thicknesses) would be one which has a low P-wave velocity layer $(\sim 8.3 \mathrm{~km} / \mathrm{s})$ below the layer with a P-wave velocity of $8.7 \mathrm{~km} / \mathrm{s}$ and above the $\mathrm{R}_{0}$ reflector (Figure B.10). However in the common receiver gathers, there is no evidence for a wide-angle reflection from the top of a low P-wave velocity layer $(\sim 8.3 \mathrm{~km} / \mathrm{s})$ in between (Figure $4.2 \mathrm{a})$.

To replicate the deeper $R_{1}$ reflection onlapping onto $R_{0}$ (Figures $4.2 a, b$ ), the $V_{P}$ contrast across the $\mathrm{R}_{0}$ reflector must be positive. Raytracing illustrates this property (Figure $4.5)$, i.e. if the interval velocity increases progressively, then each reflection will become steeper and appear to onlap to shallower reflections in a travel-time plot (Figure 4.5). Similarly, if the interval velocity decreases, reflections in a travel-time plot will tend to show little or no increase in curvature and will parallel the shallower event (Figure 4.5).

Zoeppritz equations for partitioning of seismic displacements at an interface can provide information linking polarity and amplitude of a reflection to the impedance contrast across the interface [Aki and Richards, 2002]. Determining the polarity can, 


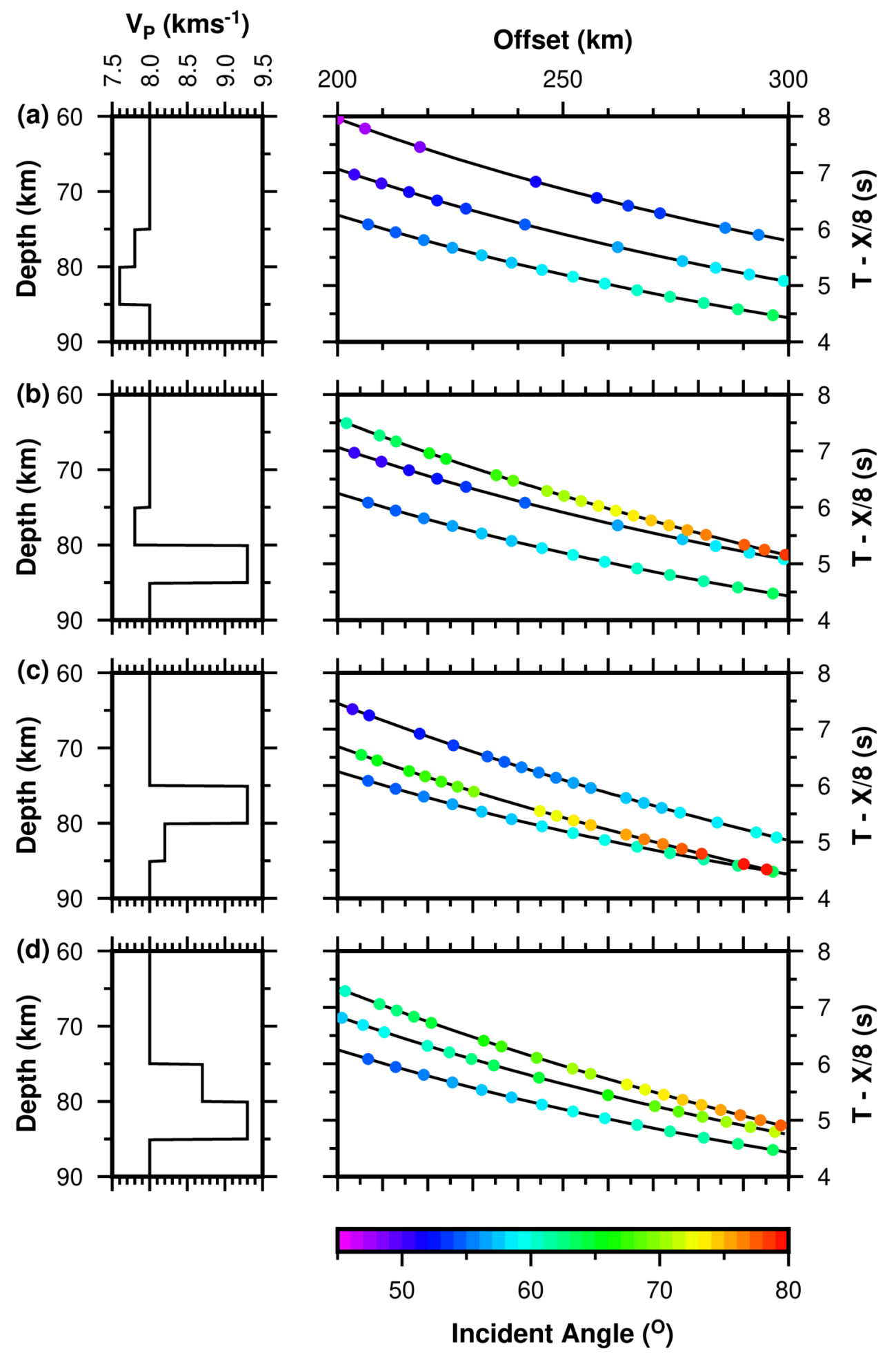

Figure 4.5 (a)-(d) One-dimensional P-wave velocity models (left) and the travel-time curves for reflections at velocity discontinuities (right) for different low- and high-velocity combinations. 
however, be perilous especially for deep, emergent looking reflections. However, we observe the following pattern in the relative polarities of the $R_{0}, R_{1}, R_{2}$ and $R_{3}$ reflections. The low-amplitude, positive first break (red deflection) for $\mathrm{R}_{0}$ (Figure $4.6 \mathrm{~b}$ ) is consistent with the impedance contrast across the boundary being positive (red has been calibrated as the positive first break based on first breaks from shallow refractions). Thus, the layer beneath $R_{0}$ has $V_{P}>9 \mathrm{~km} / \mathrm{s}$. At these depths, the mineral that can explain P-wave-velocities $>9 \mathrm{~km} / \mathrm{s}$ is consistent with olivine, which can have a maximum anisotropy of $23 \%$, with $\mathrm{V}_{\mathrm{P}} \sim 9.9 \mathrm{~km} / \mathrm{s}$ along its [100] axis at $2.5 \mathrm{GPa}$ [Zha et al., 1996]. Amplitude versus offset (AVO) modelling results for isotropic media using Zoeppritz equations and transversely isotropic media [Blangy, 1994] agree with the observed low amplitude, positive first break of $R_{0}$ within the range of the observed angles of incidence obtained from raytracing (Figure 4.7a). Synthetic waveform modelling using SPECFEM2D with a layer of olivine between $R_{0}$ and $R_{1}$ reflectors suggests that the degree of anisotropy required in the olivine layer is $\sim 13-15 \%$, corresponding to $\mathrm{V}_{\mathrm{P}}$ of $\sim 9.1-9.5 \mathrm{~km} / \mathrm{s}$ along the horizontal, and $\mathrm{V}_{\mathrm{P}}$ of $\sim 8.2-8.6 \mathrm{~km} / \mathrm{s}$ in the vertical directions (Figure B.11). Within these constraints, the thickness of this layer is $\sim 3-5 \mathrm{~km}$. Beyond these uncertainties, the merging nature of $R_{0}$ and $R_{1}$ at the 190-210 km source-receiver offset range disappears (Figure B.12).

$R_{1}, R_{2}$, and $R_{3}$ reflections show a quasi-parallel character with each other, which is the characteristic of two relatively thin, low-velocity layers (Figure 4.5). But the first break of $R_{1}$ has a positive polarity with a moderately high amplitude (Figure 4.6c) suggesting that the material below could be a layer with high velocity. However, AVO modelling suggests that the moderately high amplitude and positive polarity of $R_{1}$ within the incident angles of $58^{\circ}-70^{\circ}$ obtained from raytracing is consistent with a low P-wave velocity layer with $\mathrm{V}_{\mathrm{P}} / \mathrm{V}_{\mathrm{S}}>>2.5$ (Figure $4.7 \mathrm{~b}$ ). We estimate a P-wave velocity of $\sim 7.6 \mathrm{~km} / \mathrm{s}$ for this layer and a thickness of $\sim 5 \mathrm{~km}$ based on raytracing (Figure $4.3 \mathrm{~b}$, c), although there is no strong constraint on the velocity.

The $R_{2}$ reflection is weak and its first break appears to be blue (negative) (Figure 4.6d) whereas the first break of $\mathrm{R}_{3}$ is red (positive) (Figure 4.6e). This is consistent from AVO modelling with a slightly higher or slightly lower $V_{P}$ layer $( \pm 0.2 \mathrm{~km} / \mathrm{s})$ with a $\mathrm{V}_{\mathrm{P}} / \mathrm{V}_{\mathrm{S}}$ ratio of $\sim 1.85$ (Figure $4.7 \mathrm{c}, \mathrm{d}$ ). For example, the data are consistent with the layer below $\mathrm{R}_{3}$ being regular asthenosphere with $\mathrm{V}_{\mathrm{P}}$ of $8.1 \mathrm{~km} / \mathrm{s}$ and $\mathrm{V}_{\mathrm{P}} / \mathrm{V}_{\mathrm{S}} \sim 1.8$ [Kennett 


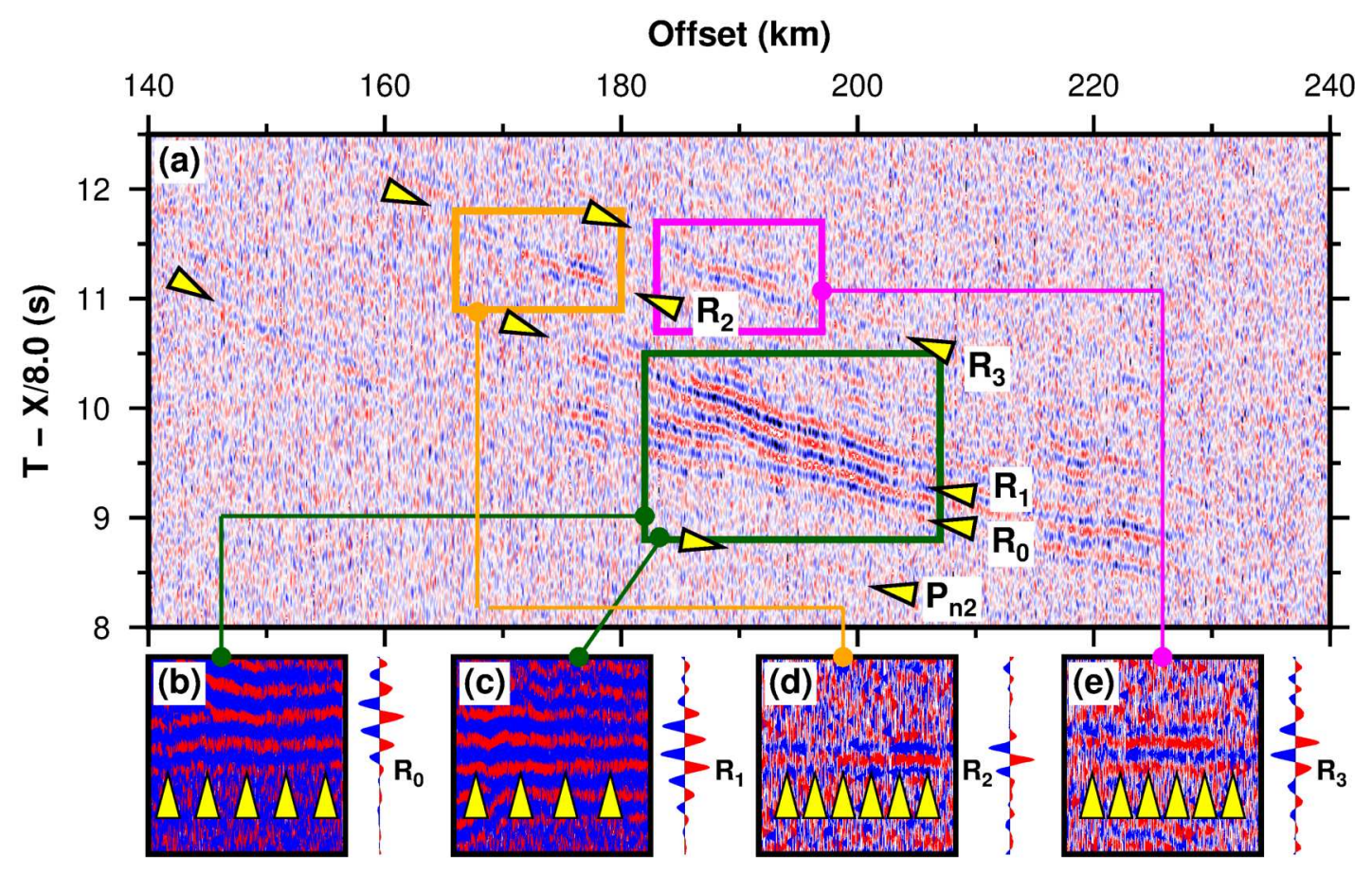

Figure 4.6 (a) Seismic phases observed in the common receiver gather at BFZ station produced using the airgun shots of SAHKE01 line. (b)-(e) Flattened $R_{0}-R_{3}$ reflections (left) and the stacked trace (right) showing the first breaks of the arrivals. First break polarity for $R_{0}-R_{3}$ are red, red, blue and red, respectively, where red is positive and blue negative polarity.

and Engdahl, 1991] (Figure 4.7e, f). The thickness of this layer between $R_{2}$ and $R_{3}$ is estimated to be $\sim 3-5 \mathrm{~km}$ from raytracing.

The depths, P- and S-wave velocities of the layers defined by the observed wide-angle reflections are summarised in Figure 4.8. The non-uniqueness of the determined Pand S-wave velocities in the preferred model could be explored using a grid-search or optimisation approach to minimise the root mean squared (RMS) amplitudes of the observed wide-angle reflections and the amplitudes calculated from Zoeppritz equations for different combinations of velocities in a top-down approach.

\subsection{Discussion}

Our results suggest the presence of a 3-5 $\mathrm{km}$ thick azimuthally anisotropic layer at a depth of $\sim 70 \mathrm{~km}$ in the lithospheric mantle that overlies a low P-wave-speed layer of thickness $\sim 8-10 \mathrm{~km}$ (Figure $4.8 \mathrm{a}, \mathrm{b}$ ). The source-receiver raypaths are in the southeast-northwest direction (Figure 4.1) implying that the top layer has a P-wave velocity of 9.1-9.5 km/s subparallel to the direction of absolute plate motion [Kreemer, 2009], and a P-wave velocity of $8.2-8.6 \mathrm{~km} / \mathrm{s}$ in the vertical direction normal to the direction of absolute plate motion. This layer potentially marks a change in anisotropy 

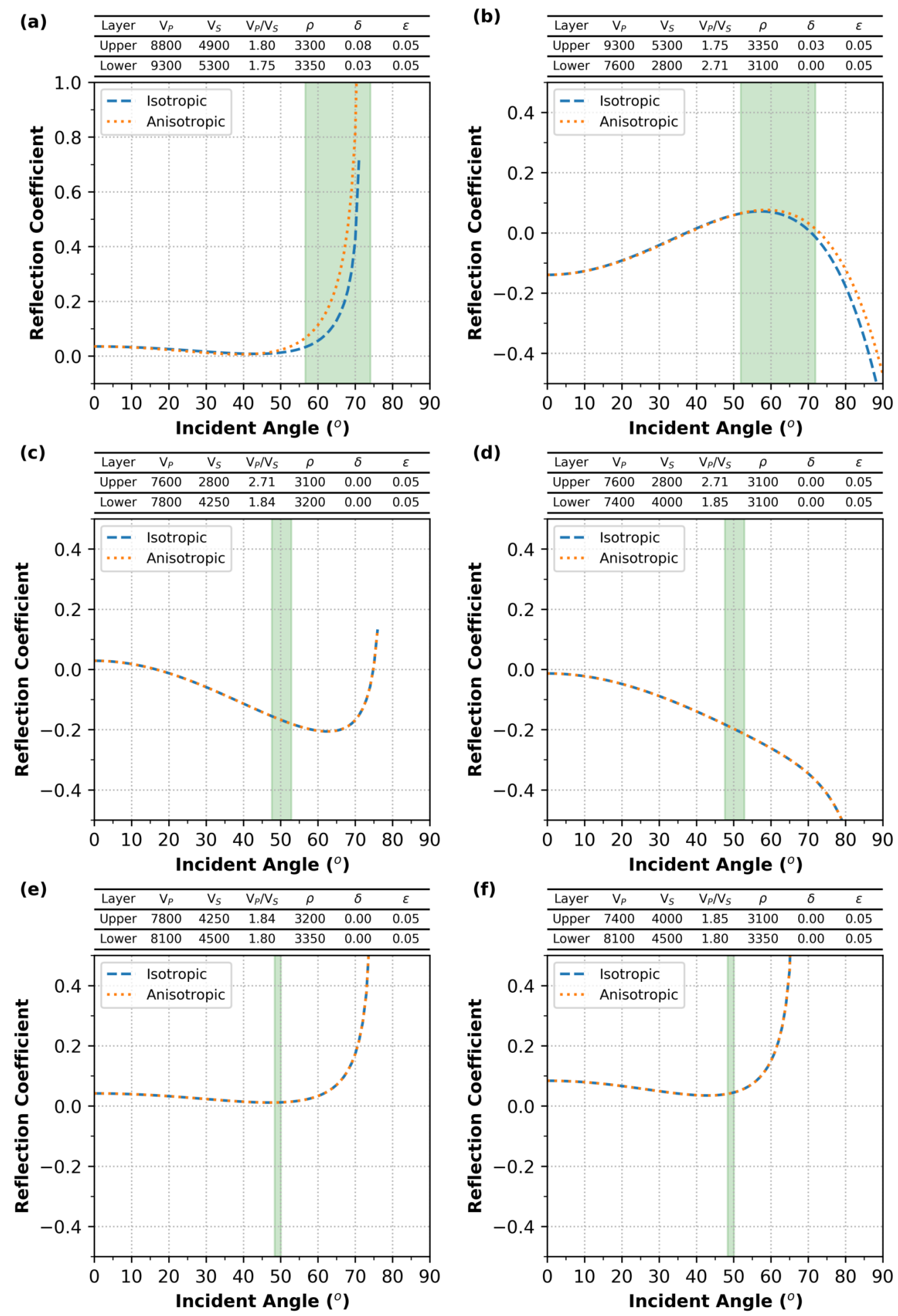

Figure 4.7 Isotropic and anisotropic P-wave reflection coefficients versus incident angles for (a) $R_{0}$, (b) $R_{1}$, (c) and (d) $R_{2}$ for two impedances of the layer below $R_{2}$ and (e) and (f) $R_{3}$ for two impedances of the layer above $R_{3}$ corresponding to $(c)$ and $(d)$. P-wave velocity $\left(V_{P}\right)$, S-wave velocity $\left(V_{S}\right)$, density $(\rho)$, and Thomsen's parameters $\delta$ and $\epsilon$ [Thomsen, 1986] for each layer are given in the table above each plot. Green shade indicates the range of incident angles for each wide-angle reflected phase obtained from raytracing. 


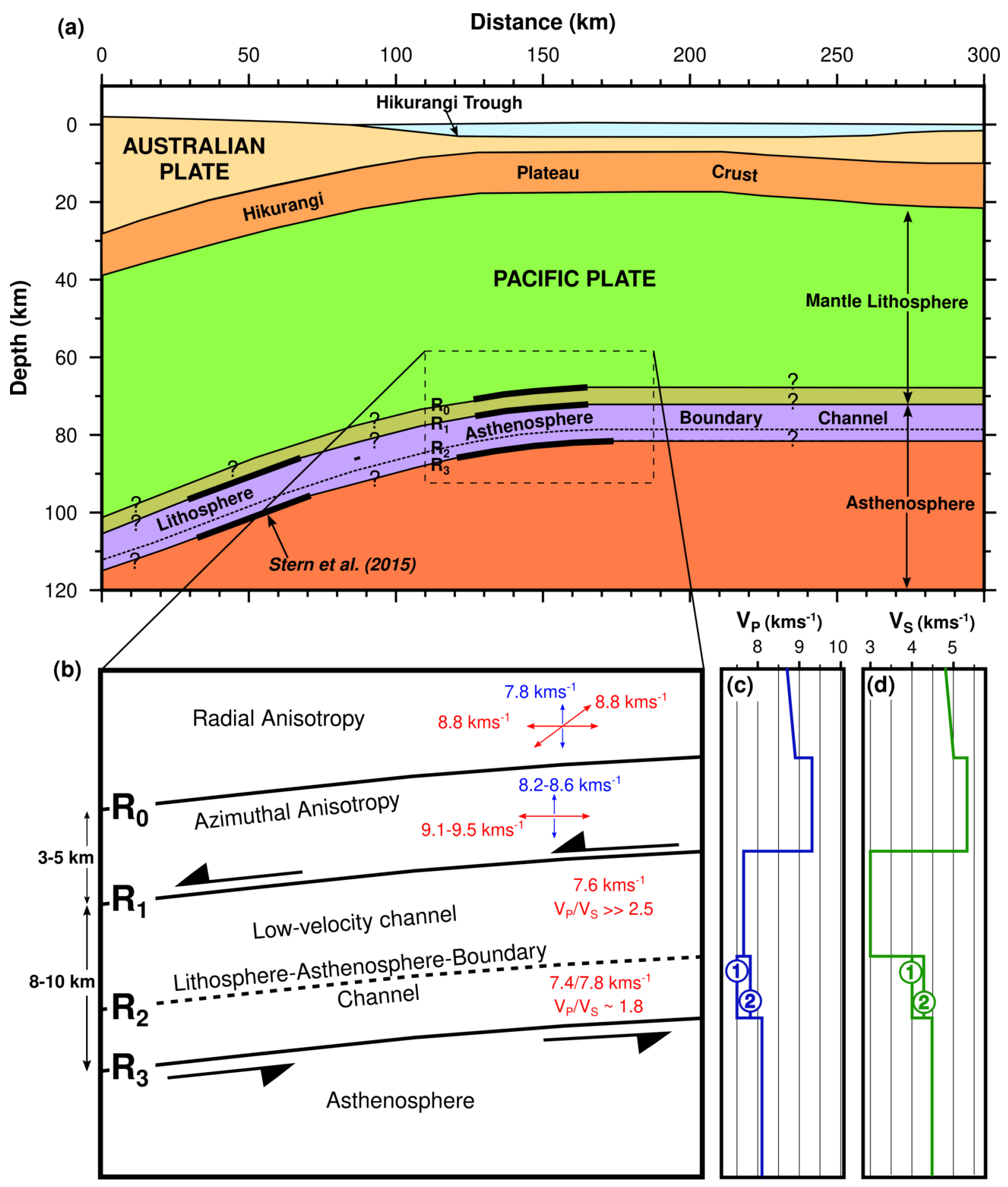

Figure 4.8 (a) Schematic diagram of the lithosphere-asthenosphere boundary zone at the Hikurangi margin. Dashed rectangle indicates the extents of (b). (b) Interpretation of the observed anisotropic and low-velocity layers. (c), (d) One-dimensional P- and S-wave velocity models for the section shown in (b). (1) and (2) indicate the possible $V_{P}$ and $V_{S}$ combinations for the layer between $R_{2}$ and $R_{3}$ reflections, obtained from AVO modelling. 
from strong radial anisotropy [Stern et al., 2020], to azimuthal anisotropy (Figure $4.8 \mathrm{~b}$ ). Below this layer, the parallel moveout of the reflections $R_{1}, R_{2}$ and $R_{3}$, and polarity of reflections, are consistent with the presence of: a $\sim 5 \mathrm{~km}$ thick layer with low P-wave velocity $(7.6 \mathrm{~km} / \mathrm{s})$ and a high $\mathrm{V}_{\mathrm{P}} / \mathrm{V}_{\mathrm{S}}$ ratio $(>>2.5)$; and a $\sim 3-5 \mathrm{~km}$ thick layer with slightly lower $(7.4 \mathrm{~km} / \mathrm{s})$ or higher $(7.8 \mathrm{~km} / \mathrm{s})$ P-wave velocity and a regular $\mathrm{V}_{\mathrm{P}} / \mathrm{V}_{\mathrm{S}}$ ratio $(\sim 1.85)$ (Figure 4.8$)$. The higher $\mathrm{V}_{\mathrm{P}} / \mathrm{V}_{\mathrm{S}}$ ratio in the upper layer indicates that it is fluid-rich, containing either melt or volatiles, whereas the relatively low $\mathrm{V}_{\mathrm{P}} / \mathrm{V}_{\mathrm{S}}$ ratio in the lower layer may indicate a relatively lower fluid content. This 8-10 km thick, low $V_{P}$ layer is interpreted to be the lithospshere-asthenosphere boundary channel of the Pacific Plate as it is consistent with the down-dip extension of the same channel found by Stern et al. [2015]. This is proposed to be a melt- or water-rich channel, with low-viscosity and higher strain rates. The depth of this low-velocity layer also agrees well with the depth to the lithosphere-asthenosphere boundary channel for a 120 Myr old oceanic plate of 70-80 km [Fischer et al., 2010; Kawakatsu et al., 2009]. Receiver function studies have also found that in the LAB channel, both P- and S- wave velocities decrease, which is attributed to a melt-rich channel [e.g. Kawakatsu et al., 2009; Rychert et al., 2010]. Further evidence for the existence of a melt-rich channel at the base of the lithosphere has come from the observation of a high-conductivity layer from magnetotelluric data [Naif et al., 2013], and from ultra-deep multichannel seismic reflection imaging [Mehouachi and Singh, 2018]. Previous controlled-source seismic studies [Mehouachi and Singh, 2018; Stern et al., 2015], with near vertical reflections detected a low $V_{P}$ layer but did not see evidence for the thin high $\mathrm{V}_{\mathrm{P}}$ layer above it. This is explicable with regard to AVO modelling that predicts only wide-angle reflections will produce reflectivity values $>0.1$ for reflections from the top of the anisotropic layer (Figure $4.7 \mathrm{~b}$ ).

Early long-range seismic reflection and refraction studies [Bean and Jacob, 1990; Díaz et al., 1993; Fuchs, 1979; Hess, 1964; Hirn, 1977; Lund, 1979; Raitt et al., 1971] have also raised the possibility of high $\mathrm{V}_{\mathrm{P}}$, anisotropic layers at depths of $60-100 \mathrm{~km}$ in the upper mantle. Anisotropy detected in these studies are confined to layers that are less than $5 \mathrm{~km}$ thick and are separated by low-velocity layers attributed to be shear zones [Bean and Jacob, 1990]. Hirn [1977] suggests that a $~ 5 \mathrm{~km}$ thick anisotropic layer with a P-wave velocity of $8.5 \mathrm{~km} / \mathrm{s}$ is located at depths near the lithosphere-asthenosphere boundary where the differential horizontal motion between the lithosphere and the 
asthenosphere occurs. This zone between the lithosphere and the asthenosphere is the most long-standing and pervasive shear zone on Earth, which can accumulate large finite shear strains. Modelling of seismic anisotropy in the upper mantle developed due to the resistive drag of the sublithospheric mantle [Tommasi, 1998] suggests that strain is localised in a horizontal shear zone several tens of kilometres thick between the rigid lithosphere and the asthenosphere. The thickness of the deformed layers depends on the displacement and the velocity of the plate. For a similar displacement, faster plates accumulate larger shear strains over thinner and shallower layers [Tommasi et al., 1996]. For a 100 million year old plate moving at a velocity of 30-60 $\mathrm{mm} /$ year, similar to the Pacific Plate at the Hikurangi margin, maximum finite shear strains of 20-25 can be encountered at depths ranging from 40-80 km. However, to produce the P-wave anisotropy of $13 \%-15 \%$ in the azimuthally anisotropic layer with P-wave speeds of $9.1-9.5 \mathrm{~km} / \mathrm{s}$ subparallel to the direction of absolute plate motion, shear strains of $2 \%-4 \%$ are sufficient [Tommasi, 1998]. The thickness of the deformed layer at these depths of $40-80 \mathrm{~km}$ from forward modelling of seismic anisotropy is between 10 and $20 \mathrm{~km}$ [Tommasi, 1998; Tommasi et al., 1996]. Cooling of the plate with age induces freezing of the upper part of the deformed layer and the fabric responsible for the measured anisotropy observed in the oceanic lithosphere is contained in two distinct layers: a frozen lithospheric layer and an asthenospheric layer [Tommasi, 1998]. The anisotropic and the low P-wave velocity layers we have detected in this study are consistent with this frozen lithospheric and asthenospheric layers. Therefore, we suggest the strong azimuthal anisotropy in the layer above the $\mathrm{LAB}$ channel we have detected in this study is a result of the reorientation of olivine crystals in response to the strong shearing generated by the differential motion between the lithosphere and the asthenosphere at the base of the lithosphere. Our interpretation of the lithosphere-asthenosphere boundary emphasises that it cannot be simply explained by a low-velocity, melt-rich channel. It is a complex system of layers which we here term the lithosphere-asthenosphere boundary zone (LABZ).

Our study reflects the importance of using wide-angle seismic reflections to explore the fine scale structures and anisotropy in the upper mantle. Although passive-source seismic techniques, like shear-wave splitting are able to detect anisotropy in the mantle, they are not able to accurately determine the depths and thicknesses of anisotropic layers, primarily due to the longer wavelengths of the seismic waves used 
and possible contamination from anisotropy in the crust [Savage, 1999]. For example, receiver function methods are reported to achieve a maximum resolution of $\sim 11 \mathrm{~km}$ [Rychert et al., 2005]. Multichannel seismic surveys are also incapable of detecting anisotropic high wave-speed layers as the near-vertical raypaths induce only small amplitude reflections. But such experiments can identify a low-velocity layer at the base of the lithosphere [Mehouachi and Singh, 2018]. Therefore, long-range wide-angle reflection and refraction surveys allow for the detection of fine-scale layering and anisotropy in the lithospheric mantle more accurately due to the higher frequencies of the sources used and their precisely known source locations and origin times. In addition to onshore-offshore seismic surveys, ocean bottom seismic surveys with long source-receiver offsets and tuned-airgun sources may be a potential candidate in exploring fine-scale layering and anisotropy at LAB depths.

\subsection{Conclusions}

Late-arriving, wide-angle reflections in the onshore-offshore data from the southern Hikurangi margin have enabled us to look at the transition from the lithosphere to the asthenosphere of the Pacific Plate from a completely different perspective. The transition zone consists of a $\sim 3-5 \mathrm{~km}$ thick azimuthally anisotropic lithospheric layer (fast direction subparallel to the direction of absolute plate motion) and $\sim 8-10 \mathrm{~km}$ thick melt- or fluid-rich channel in the asthenosphere. Large finite shear strains accumulated due to the differential motion between the lithosphere and the asthenosphere results in the realignment of olivine crystals responsible for the interpreted azimuthally anisotropic layer. Our results provide strong evidence to suggest that the transition does not take place across a single reflector or a low-velocity channel. Instead, it occurs through a series of layers with contrasting properties. Therefore, characterising the lithosphere-asthenosphere boundary as a zone, i.e. the lithosphere-asthenosphere boundary zone (LABZ), seems more appropriate. Our results also have stressed the importance of using the full spectrum of controlled-source seismic data in imaging deeper structure of the Earth to obtain a complete picture of the structure and better understand the geodynamic processes. 


\section{Acknowledgements}

SAHKE Field Team and the crew of $M / V$ Reflect Resolution are acknowledged for their time and effort in acquiring the data used in this study. Bryant Chow is acknowledged for his comments and ideas on SPECFEM2D simulations. Aleksandr Beliaev and Adrian Benson are acknowledged for their technical support. 


\title{
Chapter 5
}

\section{$\mathrm{V}_{\mathrm{P}} / \mathrm{V}_{\mathrm{S}}$ Ratio, Material and Slip-rate Deficit Variations along Hikurangi Margin Forearc}

\begin{abstract}
We use mode-converted waves from airgun shots recorded by an array of ocean bottom seismographs along the Hikurangi margin forearc to estimate average $V_{P} / V_{S}$ ratios of the material above the subducting crust. The top layer of sediments at the seafloor has average $\mathrm{V}_{\mathrm{P}} / \mathrm{V}_{\mathrm{S}}$ ratios ranging from $\sim 2.5 \pm 1.0$ to $6.0 \pm 2.5$ indicating that they are watersaturated and unconsolidated. Average $\mathrm{V}_{\mathrm{P}} / \mathrm{V}_{\mathrm{S}}$ ratios of the sediments and rocks above the top of the subducting crust range between 1.55 and 2.25 and show an along-strike variation. Low-average $V_{\mathrm{P}} / \mathrm{V}_{\mathrm{S}}$ ratios between $1.55 \pm 0.08$ and $1.78 \pm 0.12$ are estimated for a region of higher sedimentary thickness in the southern Hikurangi margin, which may result in a higher degree of compaction. It is also coincident with the offshore extension of the Pahau Torlesse Terrane which consists mainly of low-porosity, highly compacted, Cretaceous greywackes. In contrast, high $\mathrm{V}_{\mathrm{P}} / \mathrm{V}_{\mathrm{S}}$ ratios between $1.85 \pm 0.10$ and $2.22 \pm 0.08$ are observed in the central and southernmost regions with a relatively low sedimentary thickness, which may reflect effects of a lower degree of compaction and lithology. Furthermore, $\mathrm{V}_{\mathrm{P}} / \mathrm{V}_{\mathrm{S}}$ ratios show a weak correlation with the slip-rate deficits on the subduction thrust. Shear-wave splitting results indicate an anisotropy of $\sim 3.5 \%$ localised in the top layer of sediments beneath the seafloor and fast orientations perpendicular to the plate interface contours suggest stress-aligned fluid-filled cracks.
\end{abstract}




\subsection{Introduction}

Controlled-source seismology has traditionally been focused on developing P-wave velocity $\left(V_{P}\right)$ models of the subsurface, especially because the energy released by a controlled source contains P-waves. However, in such experiments, P- to S- mode conversions can occur when the P-waves are obliquely incident on interfaces with a significant impedance contrast. Yet, it has been a challenge for conventional multichannel seismic (MCS) reflection surveys to capture such mode-conversions due to the requirement of a double mode-conversion (from P- to S- and S- to P-) for the wave to reach hydrophones in the streamer and higher incident angles for efficient mode-conversion. This limitation can be overcome with the deployment of multicomponent ocean bottom seismographs (OBS) which record long offset arrivals $(>50 \mathrm{~km}$ ) and can potentially record both single and double mode-converted waves at the ocean bottom from airgun sources. Multicomponent OBS experiments have been successful in deriving the S-wave velocity structure beneath oceans using airgun sources in different tectonic settings [e.g. Digranes et al., 2003; Eccles et al., 2009; Farfour and Yoon, 2016; Kodaira et al., 1996; Tsuji et al., 2011].

P-wave velocity models provide useful information about the subsurface, yet they can often lead to ambiguous interpretations as P-wave velocities can be affected significantly by the presence of pore water. As S-waves cannot propagate in water, $\mathrm{S}$-wave velocities $\left(\mathrm{V}_{\mathrm{S}}\right)$ are observed to be even more responsive to changes in porosity, clay-content and effective stress than P-wave velocities [e.g. Castagna et al., 1985; Eberhart-Phillips et al., 1989; Lee, 2003]. Therefore, it is often desired to understand the S-wave velocities in sedimentary environments where the presence of water/fluids can play a major role in controlling the tectonic behaviour. For example, the presence of fluids reduces the effective normal stress on faults and thus reduces its frictional strength [Moreno et al., 2014]. S-wave velocity information is also more important than P-waves when performing dynamic rupture simulations of earthquakes and predicting associated ground shaking [e.g. Joyner, 2000; Kaneko et al., 2019].

The ratio of $\mathrm{P}$-wave velocity to $\mathrm{S}$-wave velocity, commonly referred to as the $\mathrm{V}_{\mathrm{P}} / \mathrm{V}_{\mathrm{S}}$ ratio, is an important diagnostic parameter of a rock, which can be estimated using modeconverted arrivals in controlled-source seismic experiments. The $\mathrm{V}_{\mathrm{P}} / \mathrm{V}_{\mathrm{S}}$ ratio is directly related to the Poisson's ratio $(\sigma)$ (Equation 5.1) and can be used to understand the 
porosity, degree of consolidation, clay-content, effective stress, pore geometry and $\mathrm{SiO}_{2}$ content [e.g. Christensen, 1984, 1996; Eberhart-Phillips et al., 1989; Lee, 2003].

$$
\sigma=\frac{1}{2}\left[1-\frac{1}{\left[\frac{V_{\mathrm{P}}}{V_{\mathrm{S}}}\right]^{2}-1}\right]
$$

Typically, crystalline rocks and consolidated sediments have $\mathrm{V}_{\mathrm{P}} / \mathrm{V}_{\mathrm{S}}$ ratios $<1.9$ [e.g. Christensen, 1984, 1996]. In contrast, unconsolidated sediments have $\mathrm{V}_{\mathrm{P}} / \mathrm{V}_{\mathrm{S}}$ ratios $>2$ [e.g. Christensen, 1984, 1996; Zhu et al., 2020] (Figure 5.1).

The Hikurangi subduction margin off the East Coast of the North Island of New Zealand (Figure 5.2a) displays a significant along-strike variation in subduction-thrust slip behaviour. The subduction-thrust in the southern Hikurangi margin is observed to be locked, as indicated by the higher slip-rate deficits and in the northern Hikurangi margin subduction thrust is observed to be creeping (Figure $5.2 \mathrm{~b})$. In addition to this, deep (30-35 km) slow-slip events (SSE) occur in the southern Hikurangi margin and shallow (5-10 km) SSEs occur in the northern Hikurangi margin [Wallace, 2020; Wallace et al., 2012a].

This spatial variation is hypothesised to be due to porosity-related variation in pore-pressure [Bassett et al., 2014; Fagereng and Ellis, 2009; Kaneko et al., 2019; Wallace, 2020; Wallace et al., 2004, 2012a]. High porosity and elevated pore pressures will result in a lower effective normal stress on the subduction thrust that will facilitate stable creeping, whereas low-porosity and reduced pore-pressure result in higher effective normal stresses increasing the frictional strength of the plate interface. These variations are reflected by $\mathrm{V}_{\mathrm{P}} / \mathrm{V}_{\mathrm{S}}$ ratios. Lower $\mathrm{V}_{\mathrm{P}} / \mathrm{V}_{\mathrm{S}}$ ratios are expected in locked regions whereas higher $V_{P} / V_{S}$ ratios are expected in creeping regions of the subduction interface.

In this study, we identify mode-converted waves from airgun shots recorded by an array of multicomponent ocean bottom seismographs along the Hikurangi margin forearc. Using these mode-converted waves, we estimate the $\mathrm{V}_{\mathrm{P}} / \mathrm{V}_{\mathrm{S}}$ ratios along the forearc to examine its relationships between material properties and slip-rate deficits at the plate interface (or plate interface coupling). 


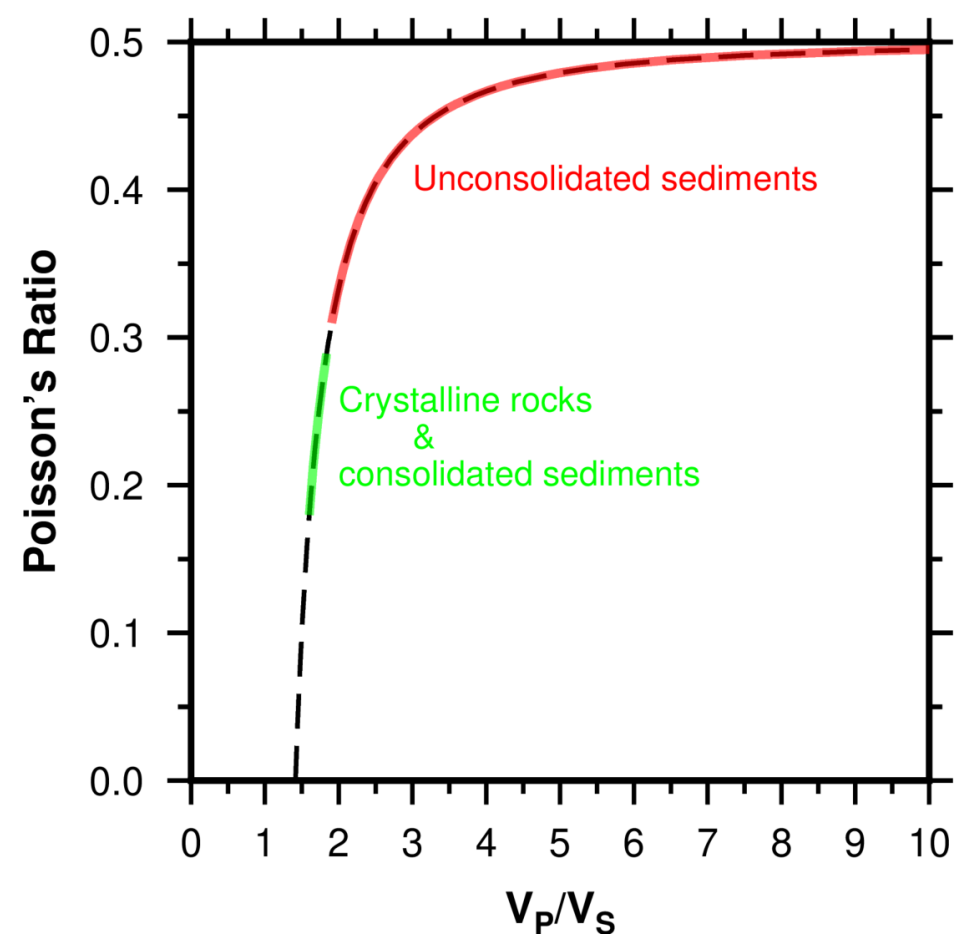

Figure 5.1 Variation of Poisson's ratio with $\mathrm{V}_{\mathrm{P}} / \mathrm{V}_{\mathrm{S}}$.

\subsection{Tectonic Setting}

Subduction of the Pacific Plate beneath the Australian Plate is marked by the Hikurangi subduction margin offshore of the East Coast of the North Island of New Zealand (Figure 5.2a). The relative plate convergence rates in the northeastern North Island are $50-60 \mathrm{~mm} /$ year and reduce to $\sim 20 \mathrm{~mm} /$ year in the southern North Island [Wallace et al., 2004]. More than $80 \%$ of the relative plate convergence occurs on the subduction thrust, with the remainder accommodated by folding and reverse faulting on the upper plate [Wallace et al., 2009, and references therein].

Geodetic modelling during interseismic time periods suggests that the plate interface in the southern Hikurangi margin is currently locked down to depths of $25-30 \mathrm{~km}$ and accumulates slip-rate deficits of up to $\sim 30 \mathrm{~mm} /$ year [Wallace et al., 2004, 2012b] (Figure $5.2 \mathrm{~b})$. The accumulated slip-rate deficit may eventually be released in a megathrust earthquake with $M_{w}>8.0$. Slow-slip events are observed at depths of $30-45 \mathrm{~km}$ in this region [Wallace et al., 2004]. In the central and northern segments of the Hikurangi margin, by contrast, the plate interface is observed to be creeping and shallow slow-slip events occur at much shallower depths (5-15 km) [Wallace et al., 2012a,b] (Figure 5.2b). A complex interplay between upper and lower plate structure, subducting sediment, thermal effects, regional tectonic stress regime, and fluid pressures have been proposed 


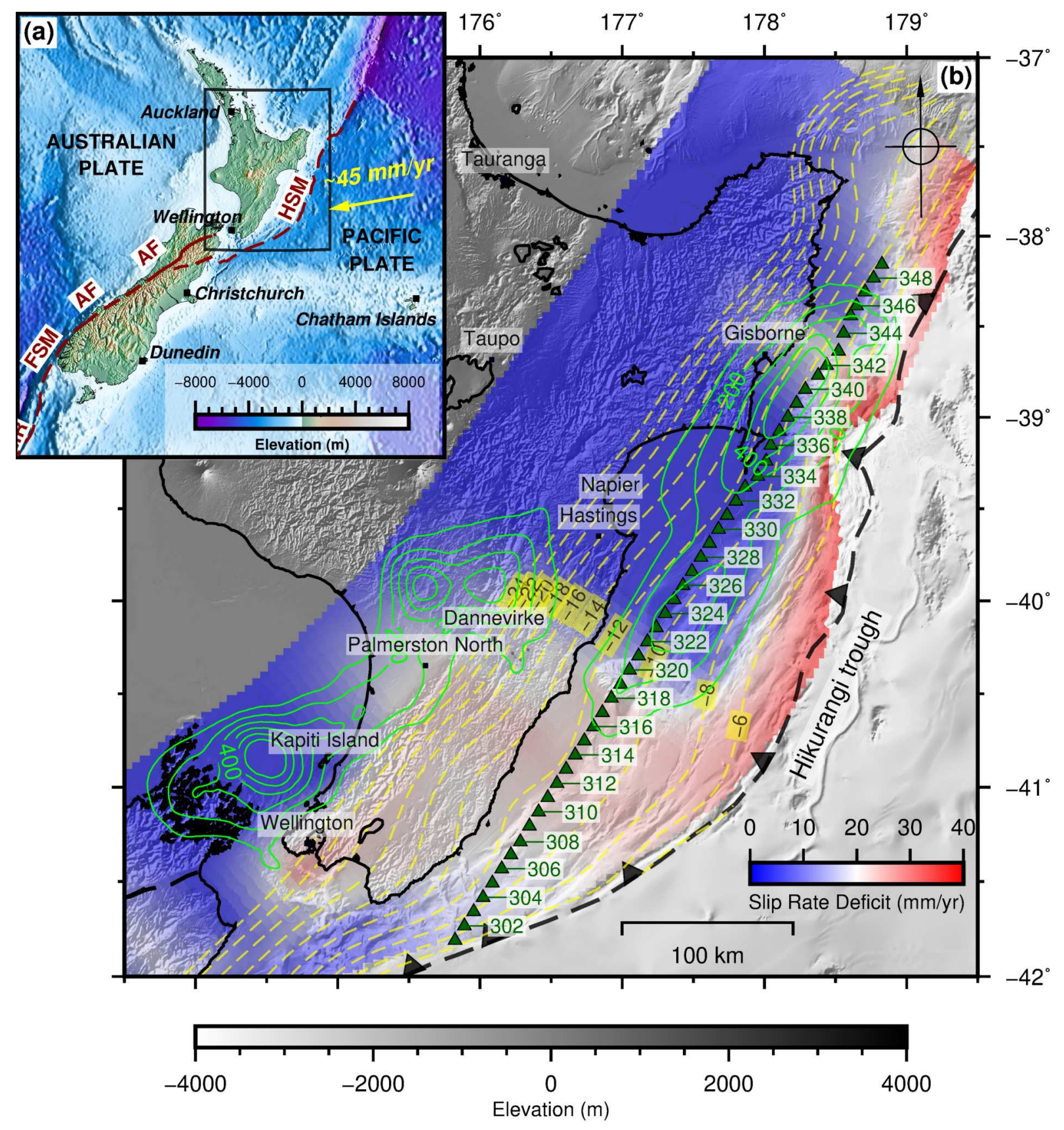

Figure 5.2 (a) Tectonic setting around New Zealand. HSM-Hikurangi subduction margin, AF-Alpine Fault, FSM-Fiordland subduction margin. Black rectangle indicates extents of (b). (b) Locations of the ocean bottom seismographs (green triangles) along the Hikurangi margin forearc from SHIRE03 profile. Green contours are slow-slip contours from Wallace et al. [2012a]. Slip-rate deficit values are from Wallace et al. [2012b]. Black, dashed-line is the Hikurangi trough. Yellow dashed lines indicates the elevation of the plate interface of Williams et al. [2013]. 
to control the extent of the subduction thrust's seismogenic zone [Wallace et al., 2009].

Furthermore, frontal accretion is observed to dominate in the southern Hikurangi margin. The accretionary wedge here is characterised by contraction indicated by folding and the presence of Torlesse Supergroup greywackes from the fossil Gondwana subduction margin [Bland et al., 2015; Mortimer, 2004]. In contrast, the central and northern segments of the margin are characterised by backarc extension, less sediment accretion and subducting seamounts [Barker et al., 2009; Bell et al., 2010].

\subsection{Data}

The Seismogenesis at Hikurangi Integrated Research Experiment (SHIRE) was conducted in 2017/2019 as a result of the joint efforts of scientists from New Zealand, United States, Japan and the United Kingdom. It comprised of the acquisition of both active- and passive-source seismic data to better understand the subsurface structure of the Hikurangi margin. Active-source seismic acquisition included the recording of offshore airgun shots at onshore seismographs and also ocean bottom seismograph (OBS) deployments along four profiles.

The $~ 528 \mathrm{~km}$ long "SHIRE03" OBS profile was deployed along the Hikurangi margin forearc (Figure 5.2b). Forty-nine (49) OBSs were deployed along this profile by $R / V$ Tangaroa at a $\sim 10-15 \mathrm{~km}$ spacing. The OBSs were provided by Japan Agency for Marine-Earth Science and Technology (JAMSTEC). These OBSs were multi-component instruments which included a triaxial, short-period seismograph (4.5 Hz natural frequency) and a hydrophone (Figure 5.3). The sampling rate was 200 $\mathrm{Hz}$ (5 ms) [Barker et al., 2019].

Airgun shots from $R / V$ Marcus G Langseth (MGL) were recorded by the array of OBS and also by the $12.75 \mathrm{~km}$ long streamer array onboard. The MGL acoustic source was comprised of four gun-strings, each with nine Bolt airguns, towed at $9 \mathrm{~m}$ depth. The airgun source chamber volume was 108 litre $\left(6,600 \mathrm{in}^{3}\right)$ and was charged to $13.1 \mathrm{MPa}$ (1900 \pm 100 Psi). The airgun shots were spaced at $\sim 50 \mathrm{~m}$ for the southwestern part of SHIRE03 and at $150 \mathrm{~m}$ for the northeastern end of SHIRE03 [Bangs, 2018; Barker et al., 2019]. 


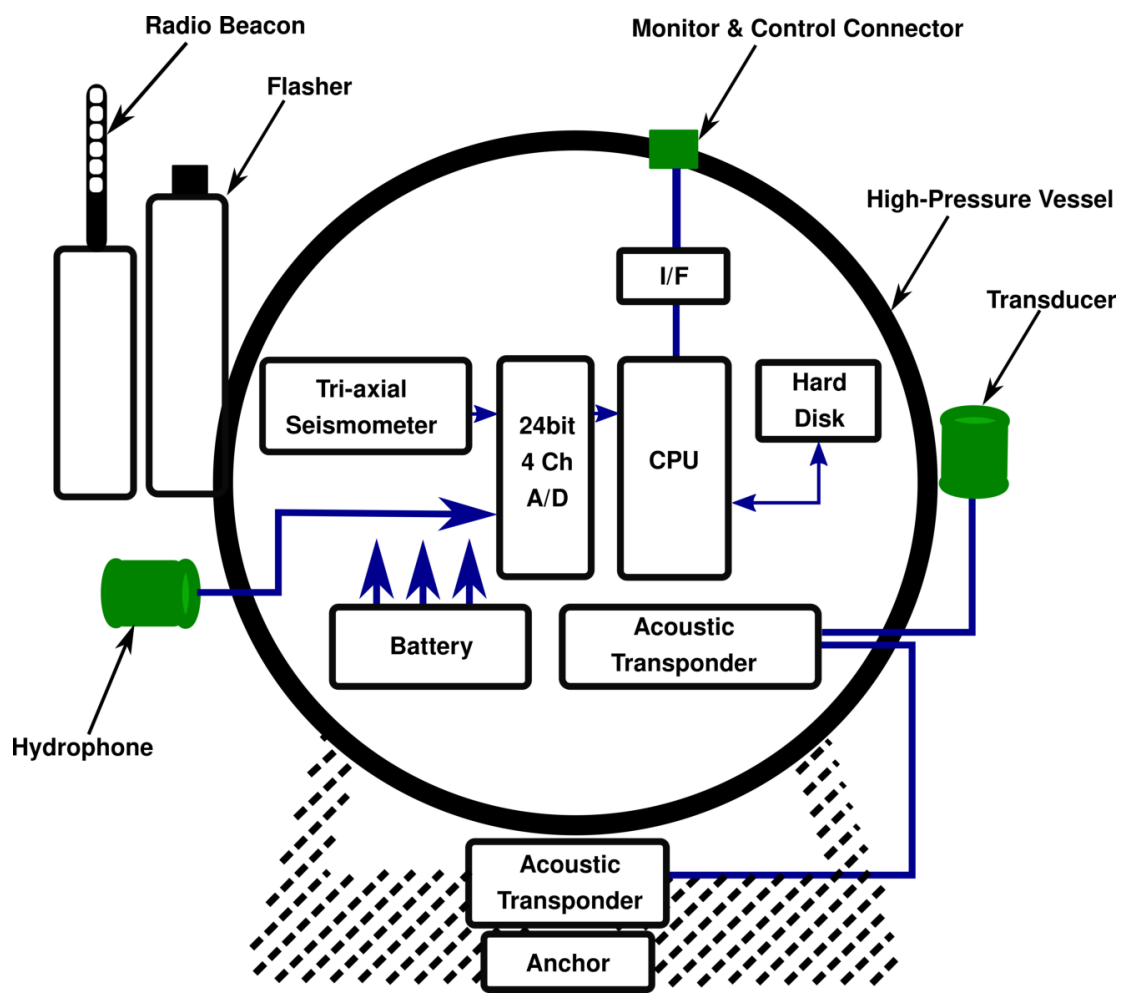

Figure 5.3 Key components of JAMSTEC OBS instruments. Modified after Barker et al. [2019].

\subsubsection{OBS Gathers}

OBS gathers, produced by cutting the continuously recorded data on OBSs based on airgun shot times, were provided by JAMSTEC with separate gathers for the vertical, two horizontal and the hydrophone components. The record length of a trace in each gather was $60 \mathrm{sec}$ at a sampling rate of $200 \mathrm{~Hz}$.

\subsection{Orienting OBS Horizontal Components}

In wide-angle modelling it is useful to look at the radial and transverse components to identify $\mathrm{S}$-waves. This is because $\mathrm{P}$ - and $\mathrm{S}_{\mathrm{V}}$-waves are polarised in the radial component and $\mathrm{S}_{\mathrm{H}}$-waves are polarised in the transverse component. In common practise, the orthogonally fixed horizontal components of a seismograph are used to rotate the seismograms into radial and transverse components based on the backazimuth to the source from the seismograph. In onshore deployments, this is straightforward as the orientation of the horizontal components are known. However, in the case of ocean bottom seismographs, which are allowed to free-fall onto the ocean bottom, the orientation of the horizontal components cannot be known without processing. 
We calculate the orientation of the horizontal components of the triaxial seismograph $\left(\mathrm{H}_{1}, \mathrm{H}_{2}\right)$ by a hodogram analysis and a polarisation analysis of the direct water arrival [Eccles, 2008]. The direct water arrival is used as it is not contaminated by any crustal signature (e.g. anisotropy) [Eccles, 2008]. The angle between positive $\mathrm{H}_{1}$ direction and the shot line can be calculated by analysing the particle motion of the direct water arrival on the horizontal components of the OBS.

The JAMSTEC OBSs used in the experiment employ a right-handed coordinate system for the gimbal-mounted triaxial seismograph. Upward movement in the vertical component $(Z)$ results in a positive voltage. Movements in the positive directions of the $\mathrm{H}_{1}$ and $\mathrm{H}_{2}$ components also result in positive voltages. In the horizontal plane (assuming that the OBS is placed perfectly level), $\mathrm{H}_{1}, \mathrm{H}_{2}$ axes and the shot line are shown in Figure 5.4.

\subsubsection{Hodogram Analysis}

A first order approximation of the $\mathrm{H}_{1}$-shot line angle is obtained by a cross plot of the particle motion of the direct water arrival between the $\mathrm{H}_{1}$ and $\mathrm{H}_{2}$ components. Figure 5.5 shows the particle motion between the $\mathrm{H}_{1}$ and $\mathrm{H}_{2}$ components with sliding time windows of length $0.2 \mathrm{sec}$ in steps of $0.1 \mathrm{sec}$, within the offset range of the direct water arrival. At far offsets of the direct water arrival, the direction of particle motion is the same as the angle between the respective component and the shot line.

A linear regression analysis of the particle motion of the direct arrival inside a time window of $0.2 \mathrm{sec}$ centred on the hand-picked direct arrival was carried out. The slope of the linear regression is equal to the angle between $\mathrm{H}_{1}$ and the shot-line (Figure 5.4). A small but evident asymmetry can be observed in the angles in Figure 5.6 at far negative and positive offsets. This is due to the deviation of the components from the horizontal plane and/or offline position of the OBS. At small offsets, the angles also deviate from those at far offsets due to the effect of small horizontal amplitudes as a result of near vertical angles of incidence [Eccles, 2008].

\subsubsection{Polarisation Analysis}

A polarisation analysis of the direct water arrival is used to further ascertain the orientations of the horizontal components of the OBS [Eccles, 2008] obtained from the above methods. Polarisation angles are calculated by the eigendecomposition of the 


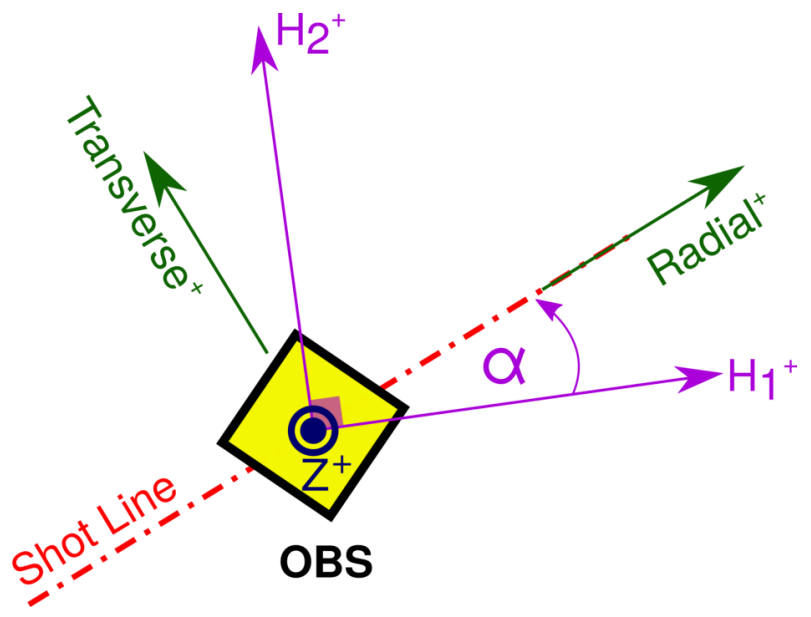

Figure 5.4 Schematic diagram showing the orientations of the positive axes of the vertical $\left(Z^{+}\right)$and two horizontal $\left(\mathrm{H}_{1}{ }^{+}, \mathrm{H}_{2}{ }^{+}\right)$components of an ocean bottom seismograph. Orientations of the positive directions of radial and transverse axes of the study are also given. $\alpha$ is the angle between the $\mathrm{H}_{1}{ }^{+}$and the Radial $^{+}$directions.

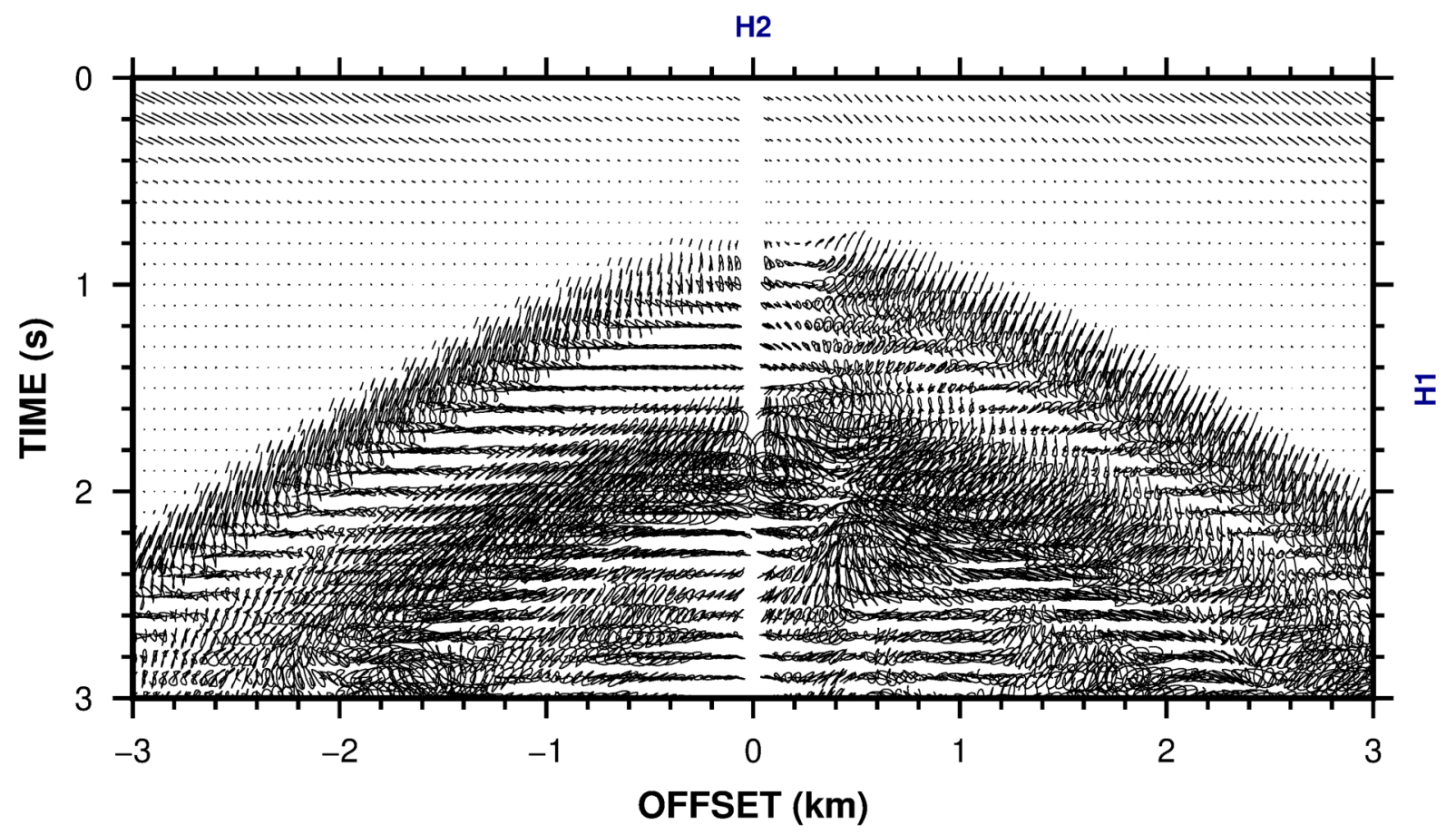

Figure 5.5 Direct water arrival hodogram of the horizontal components at OBS 309. 


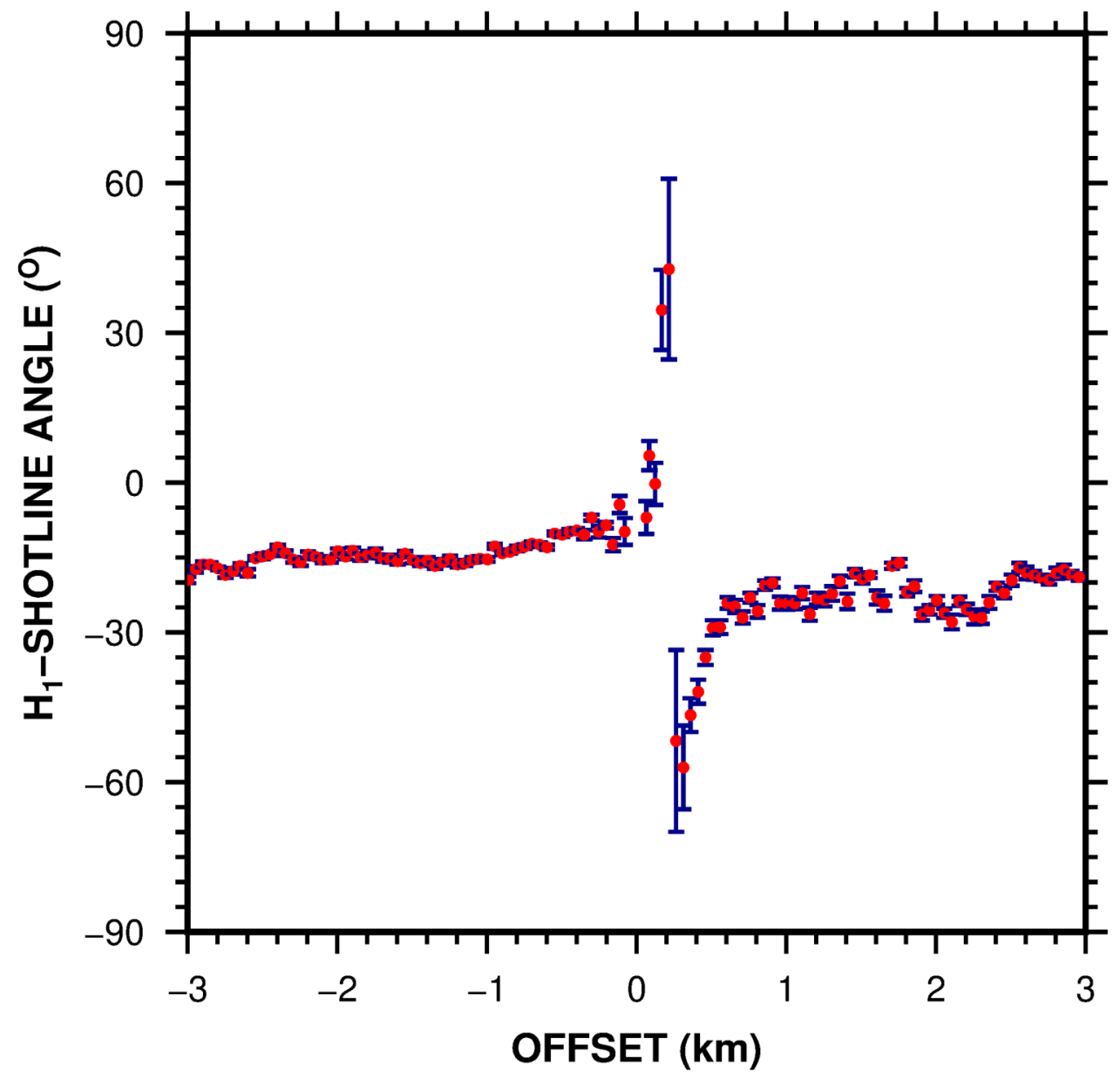

Figure 5.6 Linear regression analysis of the direct water arrival at OBS 309. Hodogram shown in Figure 5.5 .

covariance matrix of the three components of the OBS [Flinn, 1965]. This is a $3 \times 3$ matrix of cross-correlations between the three components. Eigendecomposition of this matrix provides the eigenvectors and eigenvalues of an ellipsoid that represents the trajectory of particle motion in the 3D space.

Considering a horizontal plane, the covariance can be reduced to a $2 \times 2$ matrix. Eigendecomposition of this matrix provides the eigenvectors and eigenvalues of an ellipse that represents the trajectory of the particle motion on the 2D horizontal plane. Eigendecomposition of $\mathrm{H}_{1}$ and $\mathrm{H}_{2}$ components in the offset range of the direct water arrival was conducted within sliding time windows of $50 \mathrm{msec}$. The polarisation angles are calculated from the inverse tangent of the corresponding eigenvectors. The polarisation angle of the direct water arrival is similar to the $\mathrm{H}_{1}$-shot line angle (Figure 5.7). 


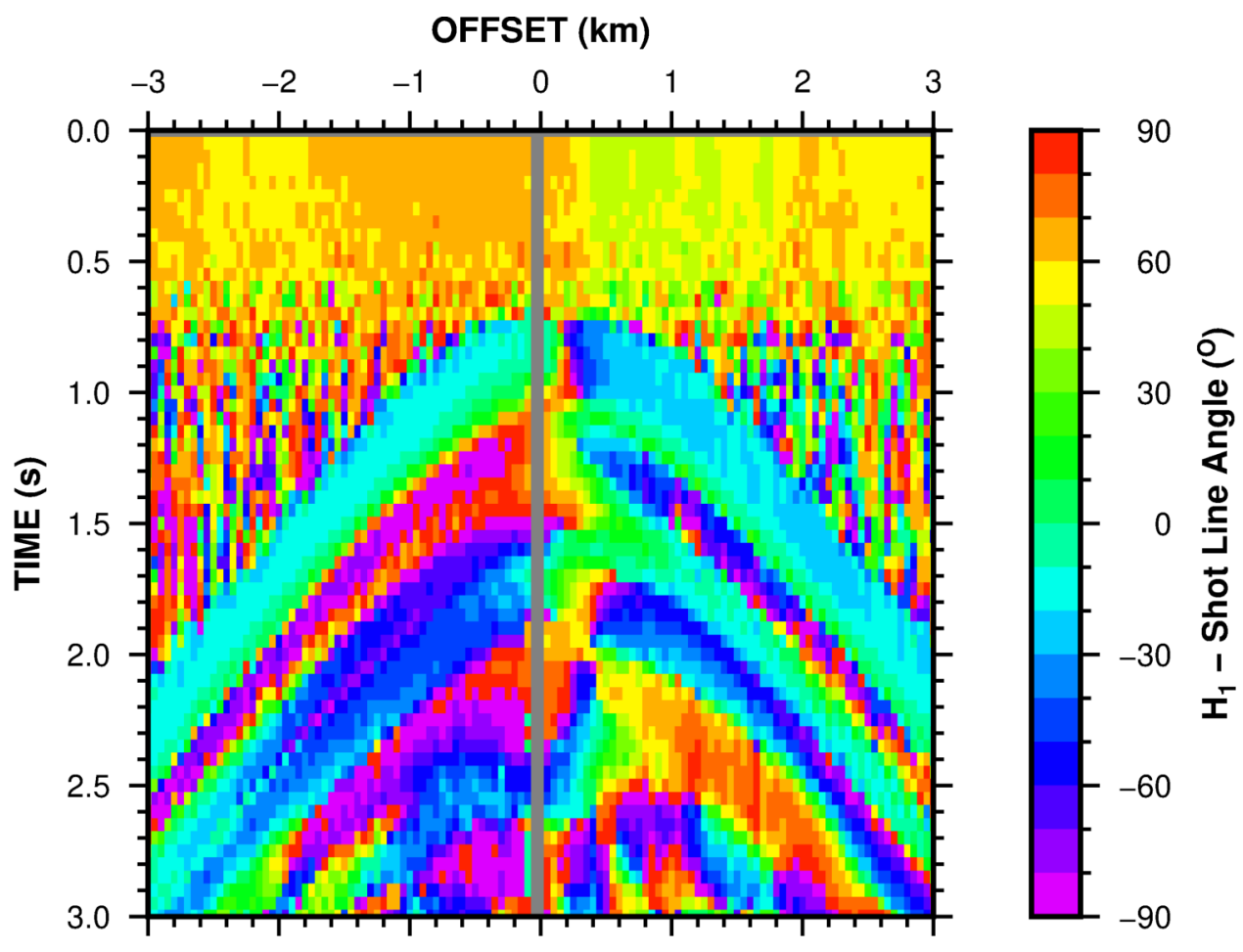

Figure 5.7 Polarisation angles in the horizontal plane determined from the eigendecomposition of covariance matrix of the two horizontal components at OBS 309. The polarisation angle of the direct water arrival is similar to the angle between the $\mathrm{H}_{1}{ }^{+}$and the shot-line.

\subsubsection{Resolving Ambiguity}

The angle between $\mathrm{H}_{1}$ and the shot line determined from the above methods inherently has an uncertainty of about $\pm 10^{\circ}$. In addition, the angle also has a $180^{\circ}$ ambiguity. To resolve this ambiguity, a further analysis was done by rotating the two horizontal components into radial and transverse directions using the angle determined from the linear regression method and its $180^{\circ}$ ambiguous angle.

Simple algebraic solutions can be obtained for the radial and transverse components (Equations 5.2 and 5.3) based on the trigonometrical relationships in Figure 5.4. When the rotation into radial and transverse components has been performed with the correct angle, particle motion of the direct water arrival in between the radial and vertical components should have a "christmas tree" like pattern (Figure 5.8). For example, the correct angle between $\mathrm{H}_{1}$ and shot line at OBS 309 is $-18^{\circ}$ (Figure 5.9).

$$
\text { Radial }=\mathrm{H}_{1} \cos \alpha+\mathrm{H}_{2} \sin \alpha
$$




$$
\text { Transverse }=-\mathrm{H}_{1} \sin \alpha+\mathrm{H}_{2} \cos \alpha
$$

A further check to see if the rotation has been performed properly was done by plotting the particle motion of the direct arrivals of the vertical, radial and transverse components (Figure 5.10). When the rotation is correct, particle motion should be polarised in the radial component, and not in the transverse component (Figure 5.10b, c).

Using these methods, orientations of the horizontal components were calculated (Table 5.1). However, orientations for all OBSs could not be calculated due to lower signal-tonoise ratio (in some OBSs particularly in the north), shallower water depths and large offline distances which would not register the direct water arrival as the first arrival.

\subsubsection{Rotating to Radial and Transverse Components}

After resolving the ambiguity of the orientation of the horizontal components of each OBS, they were rotated to their radial and transverse components, based on Equations 5.2 and 5.3. Figure 5.11 shows the vertical, radial, transverse and hydrophone components for OBS 309.

\subsection{Processing OBS gathers}

The rotated gathers were processed to increase the signal-to-noise ratio to assist identify and distinguish between direct and mode-converted arrivals. The processing was carried out using GLOBE Claritas $^{T M}$ seismic processing software.

A zero-phase, bandpass filter of corner frequencies 2-4-10-15 Hz was applied. Predictive deconvolution using a filter length of $0.7 \mathrm{sec}$ and a gap length of $0.5 \mathrm{sec}$ was applied to reduce "ringiness" of the arrivals (Section 2.4.2). Furthermore, runmixing (Section 2.4.2) was used to boost up the signal-to-noise ratio where weak coherent signals were observed.

\subsection{Identification of Mode-converted Phases}

Several types of mode-converted waves can be expected in wide-angle data recorded by OBSs (Figure 5.13). PSS mode-conversions occur when the P-wave from the airgun 
Table 5.1 Orientations of the horizontal components of OBS stations. $\mathrm{H}_{1}$-shot line angles in degrees for each OBS. Azimuths of $\mathrm{H}_{1}$ and $\mathrm{H}_{2}$ are in degrees positive clockwise.

\begin{tabular}{|c|c|c|c|}
\hline OBS Station & $\mathrm{H}_{1}$-shotline angle & $\mathrm{H}_{1}$ azimuth & $\mathrm{H}_{2}$ azimuth \\
\hline 301 & 46 & 81 & 351 \\
\hline 302 & 128 & 163 & 73 \\
\hline 303 & 151 & 186 & 96 \\
\hline 304 & 136 & 171 & 81 \\
\hline 305 & 131 & 166 & 76 \\
\hline 306 & 38 & 73 & 343 \\
\hline 307 & -75 & 320 & 230 \\
\hline 308 & -79 & 316 & 226 \\
\hline 309 & -18 & 17 & 287 \\
\hline 310 & -42 & 353 & 263 \\
\hline 311 & 131 & 166 & 76 \\
\hline 312 & 149 & 184 & 94 \\
\hline 313 & 48 & 83 & 353 \\
\hline 314 & -44 & 351 & 261 \\
\hline 315 & 66 & 101 & 11 \\
\hline 316 & 143 & 178 & 88 \\
\hline 317 & 226 & 261 & 171 \\
\hline 318 & 218 & 253 & 163 \\
\hline 319 & 189 & 224 & 134 \\
\hline 320 & -15 & 20 & 290 \\
\hline 323 & -64 & 331 & 241 \\
\hline 324 & 39 & 74 & 344 \\
\hline 326 & 231 & 266 & 176 \\
\hline 327 & 172 & 207 & 117 \\
\hline 328 & 44 & 79 & 349 \\
\hline 329 & -15 & 20 & 290 \\
\hline 331 & 53 & 88 & 358 \\
\hline 337 & 75 & 110 & 20 \\
\hline 338 & 18 & 53 & 323 \\
\hline 339 & 54 & 89 & 359 \\
\hline 340 & 67 & 102 & 12 \\
\hline 341 & 204 & 239 & 149 \\
\hline
\end{tabular}



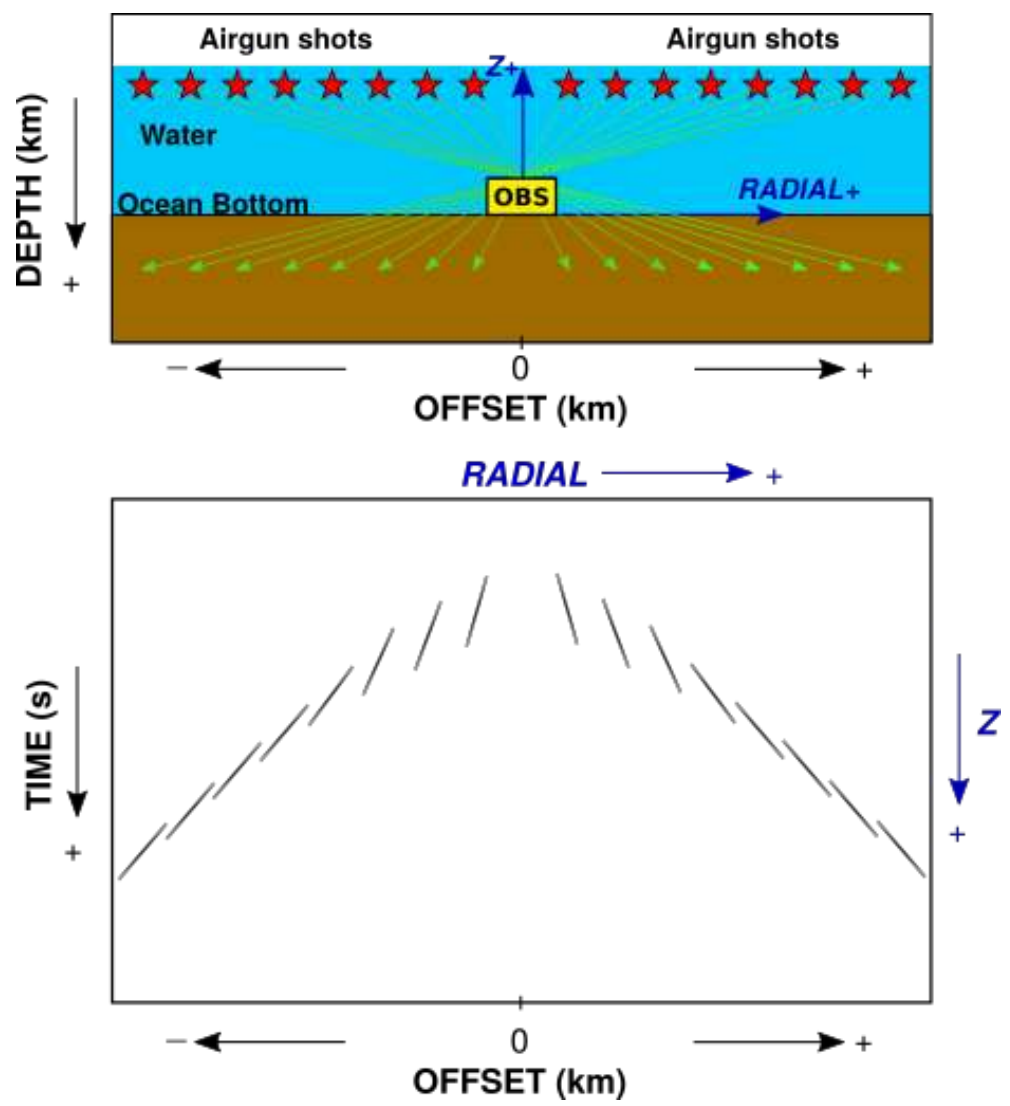

Figure 5.8 Schematic diagram showing the orientation of the particle motions of the direct arrival in the radial and vertical $(Z)$ components, when the $\mathrm{H}_{1}$ and $\mathrm{H}_{2}$ components have been rotated to their radial and transverse components using the correct rotation angle.

shot converts to an S-wave at an interface on its way down, and arrives at the OBS as an S-wave. PPS mode-conversions occur when a refracted wave from an airgun shot converts to an S-wave on its way up and arrives at the OBS as an S-wave. A double mode-conversion referred to as PSP occurs when the downgoing P-wave converts to an $\mathrm{S}$-wave, refracts as an S-wave, converts again to a $\mathrm{P}$-wave on its way up and arrives as a P-wave at the OBS (Figure 5.13).

In the rotated radial and transverse components, identification of mode-converted waves arriving at the OBS stations in Table 5.1 were conducted. For this purpose, polarisation angles and rectilinearity (ellipticity) of arrivals, linear moveout, comparison with vertical and hydrophone components were used.

\subsubsection{Polarisation Angles and Rectilinearity}

As outlined in Section 5.4.2, eigendecomposition of the covariance matrix of the triaxial seismograph components of the OBS provides eigenvectors and eigenvalues of an ellipsoid that represents the trajectory of particle motion in the 3D space. The eigenvectors representing the particle motion along the horizontal components are 
(a) Shot Line $-\mathrm{H}_{1}$ Angle : $-18^{\circ}$

\section{Radial}

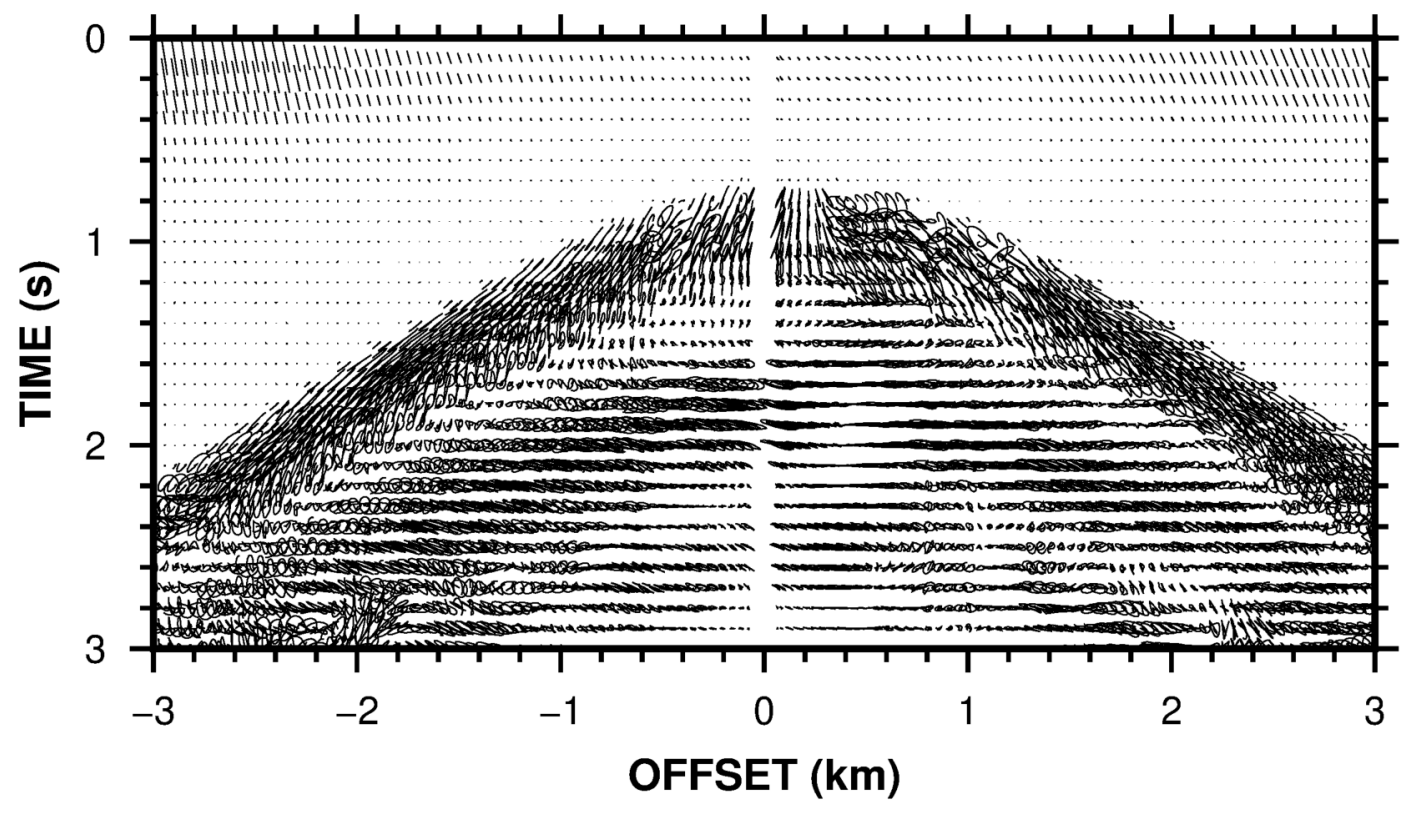

(b) Shot Line $-\mathrm{H}_{1}$ Angle : $162^{\circ}$

\section{Radial}

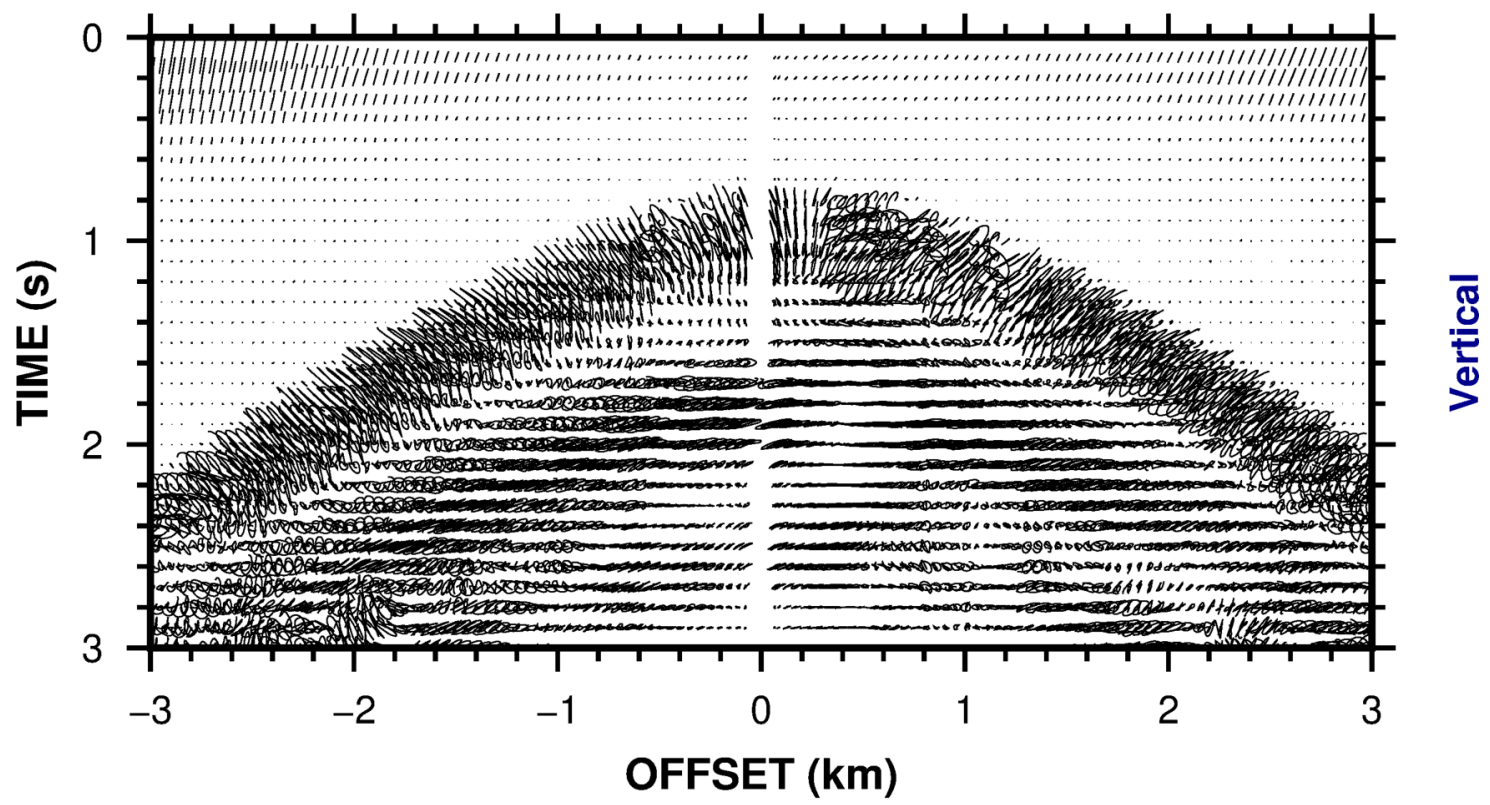

Figure 5.9 Hodograms of radial versus vertical components rotated using (a) $\mathrm{H}_{1}$-shot line angle $=-18^{\circ}$ and (b) $\mathrm{H}_{1}$-shot line angle $=162^{\circ}$. The hodogram that shows particle motion like a "christmas tree" for the direct water arrival indicates the correct rotation angle when compared with Figure 5.8. The correct rotation angle at OBS 309 is $-18^{\circ}$. 


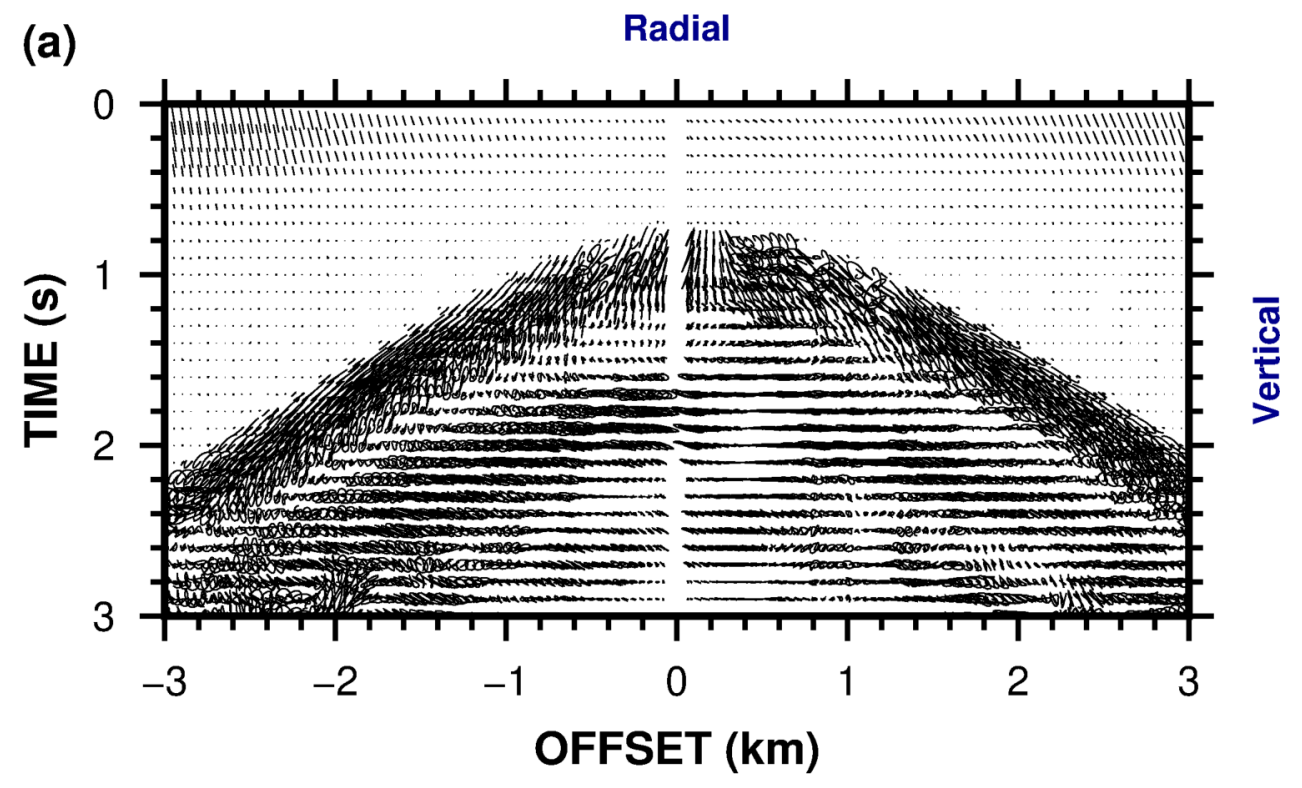

(b) Transverse

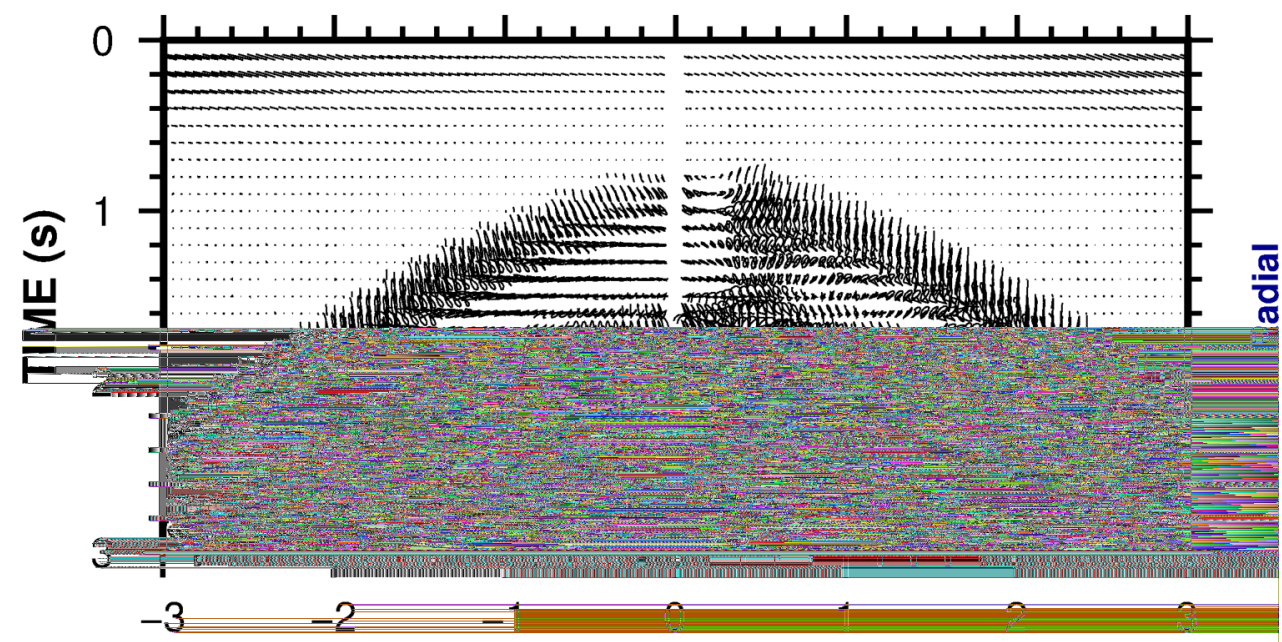

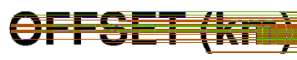
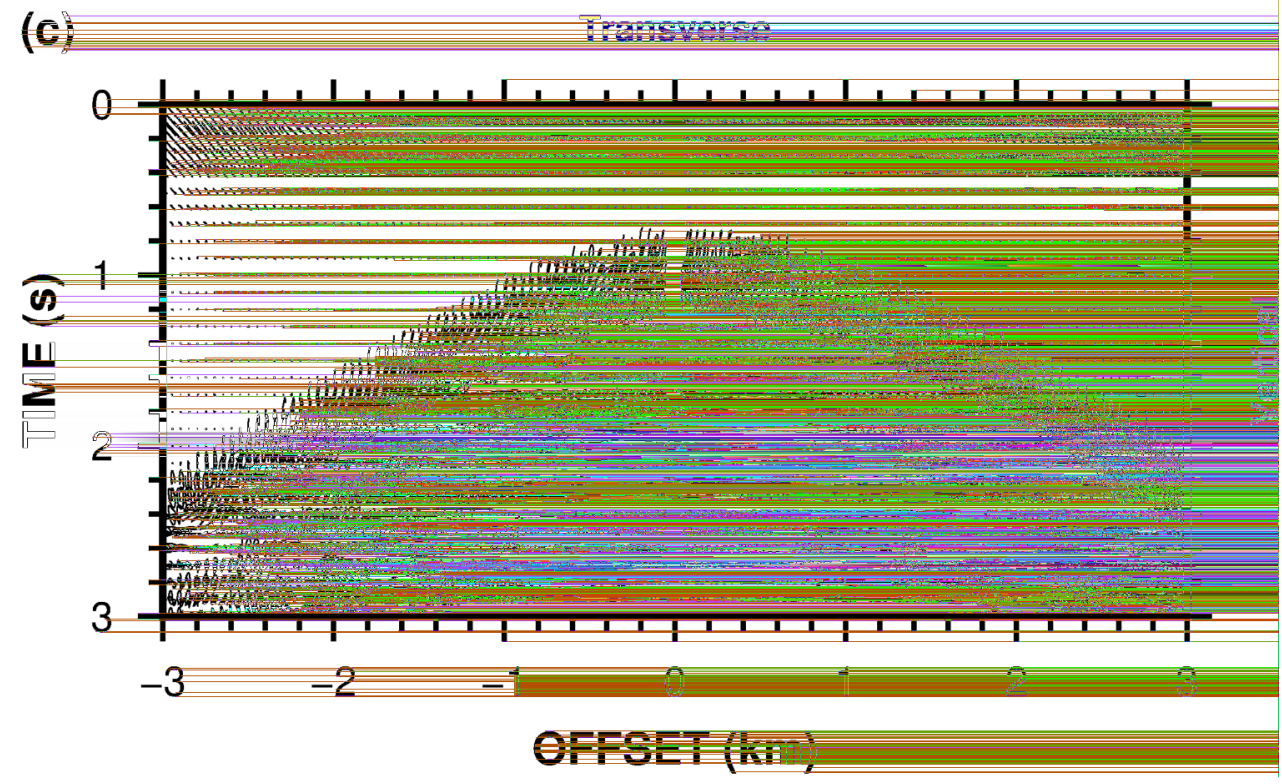

Figure 5.10 Hodograms of (a) radial versus vertical components, (b) transverse versus radial components, and (c) transverse versus vertical components after correct rotation at OBS 309. Note the polarisation of the particle motion in the radial component than the transverse component. 

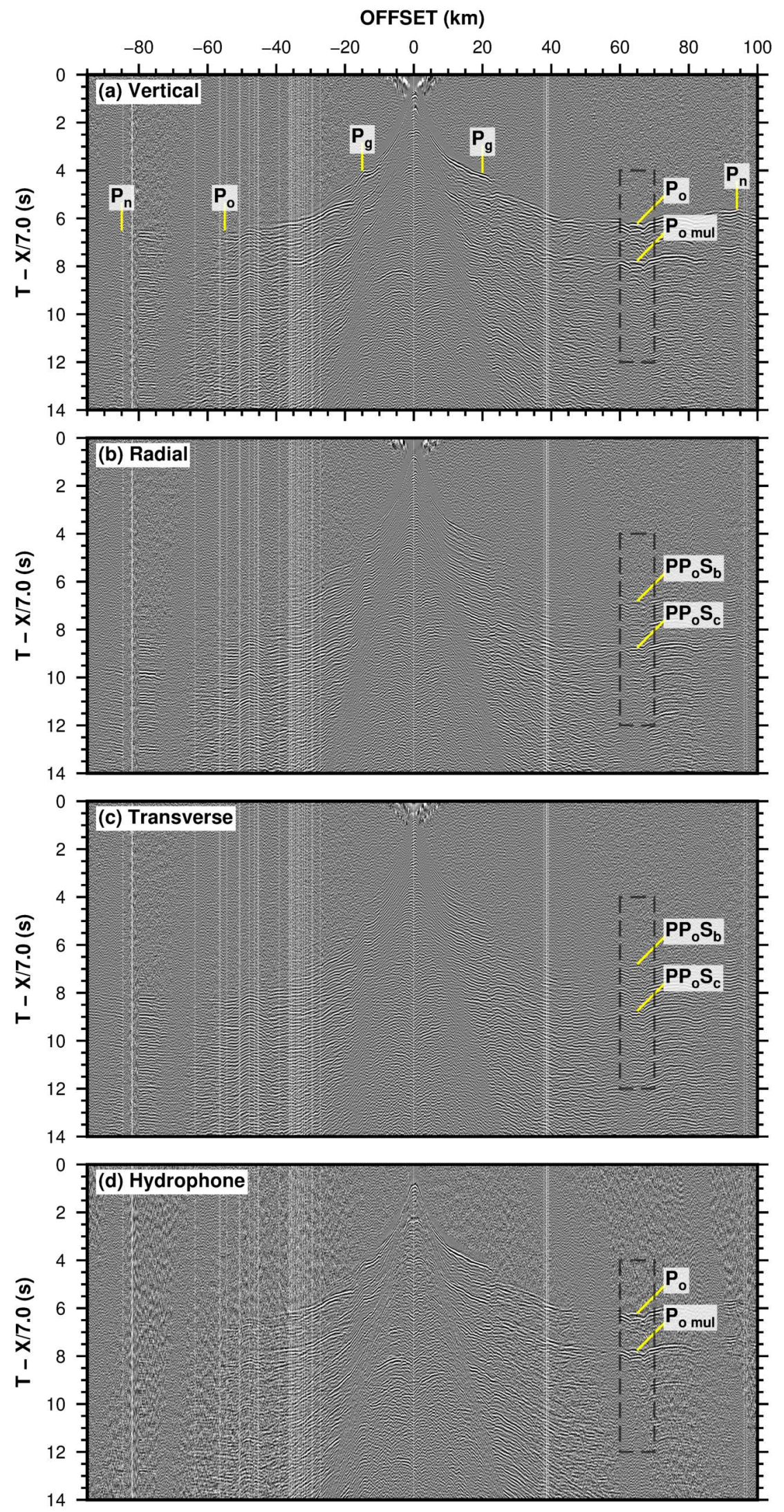

Figure 5.11 Common receiver gathers of (a) vertical, (b) radial, (c) transverse and (d) hydrophone components at OBS 309. Note that the travel-time axes are reduced using a reduction velocity of 7.0 $\mathrm{km} / \mathrm{s}$. Identified seismic phases are labelled. $\mathrm{P}_{\mathrm{g}}$-refraction in the upper plate, $\mathrm{P}_{\mathrm{o}}$-refraction in the oceanic crust, $\mathrm{P}_{\mathrm{n}}$-upper mantle refraction, $\mathrm{P}_{\mathrm{x} \text { mul }}$ indicates the water column multiple of the refractions. $\mathrm{PP}_{\mathrm{x}} \mathrm{S}_{\mathrm{b}}$ are the PPS mode-conversions from the refractions at the top-sediment basement interface (subscript " $\mathrm{b}$ "). $\mathrm{PP}_{\mathrm{x}} \mathrm{S}_{\mathrm{c}}$ are the PPS mode-conversions from the refractions at the top of the oceanic crust (subscript " $\mathrm{c}^{\prime \prime}$ ). $\mathrm{x}=\{\mathrm{g}, \mathrm{o}, \mathrm{n}\}$. Dashed rectangles indicate sections of each component zoomed in Figure 5.12. 

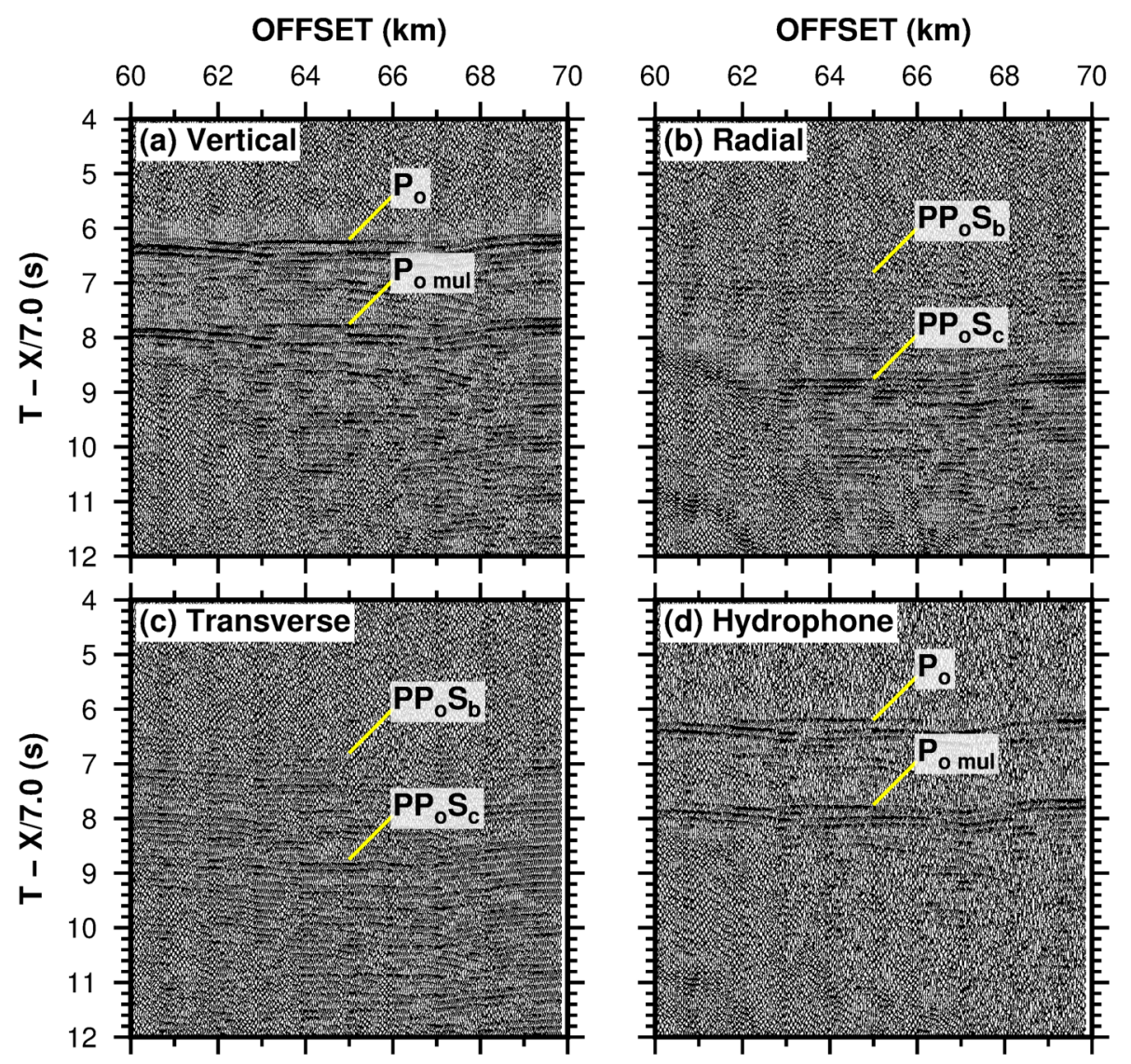

Figure 5.12 Zoomed sections of vertical, radial, transverse and hydrophone components at OBS 309. See Figure 5.11 for a description of the identified phases.

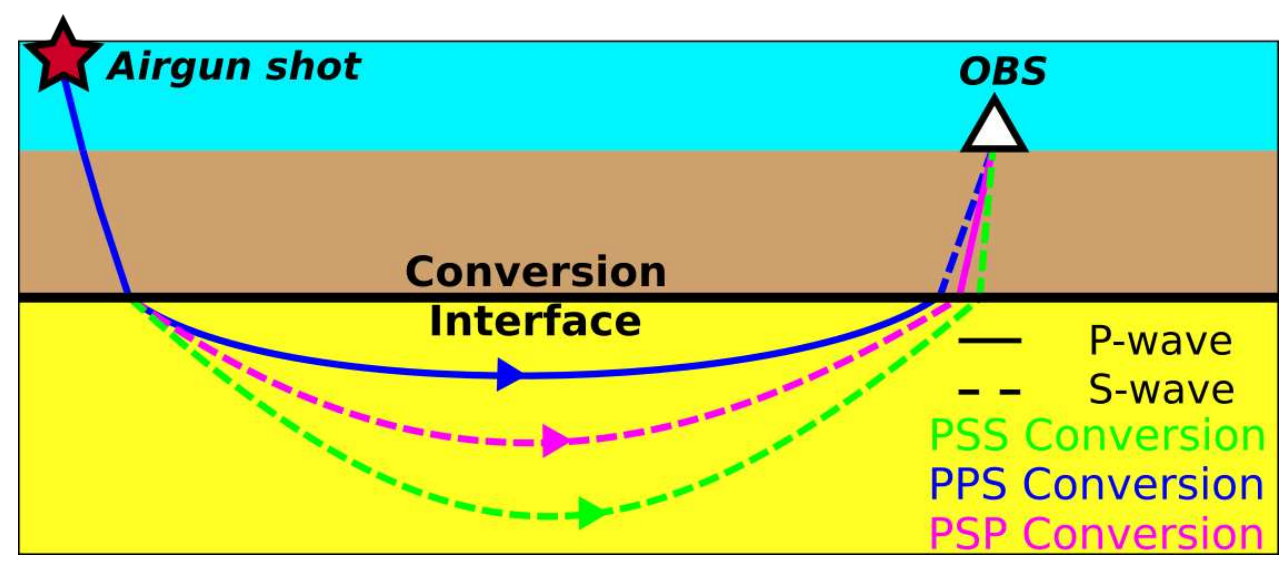

Figure 5.13 Schematic diagram showing PSS, PPS and PSP mode-conversions at an interface. 
used to calculate the resultant vector in the horizontal plane. The angle between the vertical component eigenvector and the resultant horizontal vector is a measure of the polarisation angle in the vertical plane along the shot line. Polarisation angles within $\pm 30^{\circ}$ indicate strong polarisation in the vertical component and such arrivals are more likely to be incoming P-waves, either primary arrivals or water column multiples. Polarisation angles approaching $\pm 90^{\circ}$ indicate strong polarisation in the horizontal plane, and they are more likely to represent incoming S-waves. This is particularly useful in distinguishing PPS mode-conversions (Figure 5.14a), as both $\mathrm{S}_{\mathrm{H}}$ and $\mathrm{S}_{\mathrm{V}}$ waves are likely to be polarised along the horizontal plane due to the near vertical raypaths (Figure 5.13).

The ellipticity of the particle motion can be calculated from the square root of the ratio of the largest eigenvalue to the second largest eigenvalue [Flinn, 1965]. Rectilinearity of the particle motion is defined as 1 - ellipticity [Perelberg and Hornbostel, 1994]. Since incoming P-waves to the OBS display a linear particle motion, first arriving P-waves and their multiples can be identified based on the degree of rectilinearity of arrivals (Figure $5.14 b)$.

Polarisation angles in the vertical plane and rectilinearity of arrivals for sliding windows of length $100 \mathrm{msec}$ with a step of $50 \mathrm{msec}$ were calculated to distinguish between direct arrivals, water column multiples and mode-conversions (Figure 5.14).

\subsubsection{Linear Moveout}

Application of a linear moveout flattens out refracted arrivals from a layer whose velocity is equal to the linear moveout velocity. This is particularly useful in identifying PSS and PSP mode-conversions whose gradients in a travel-time versus offset plot are inversely proportional to the S-wave velocity of the refracting layer. This helps in differentiating P-wave arrivals based on the refracting velocity.

\subsubsection{Comparison with Hydrophone Component}

The OBSs used in the study are equipped with a hydrophone that resides in the water column. As S-waves cannot propagate in water, S-waves arriving at the OBS will not be recorded by the hydrophone component. Therefore, comparing the phases observed in the hydrophone component with potential S-wave arrivals in horizontal components 


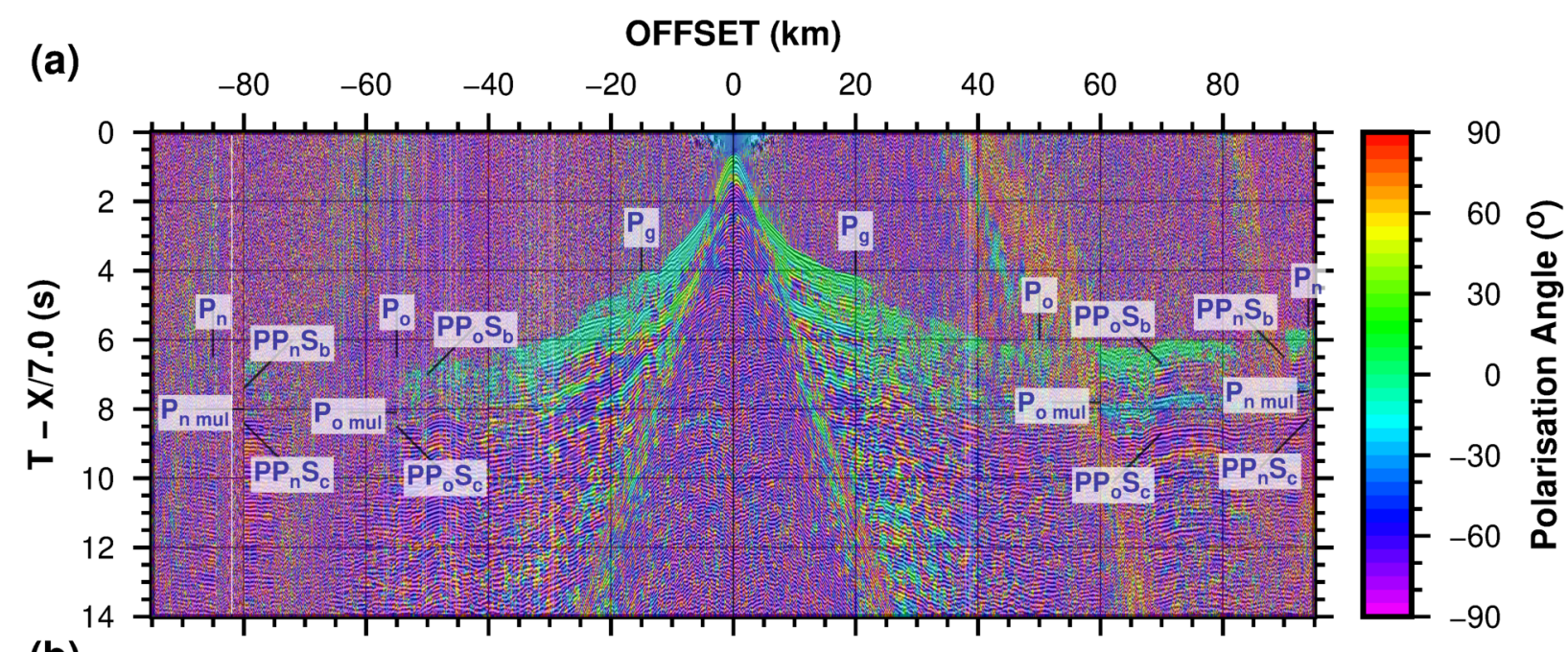

(b)

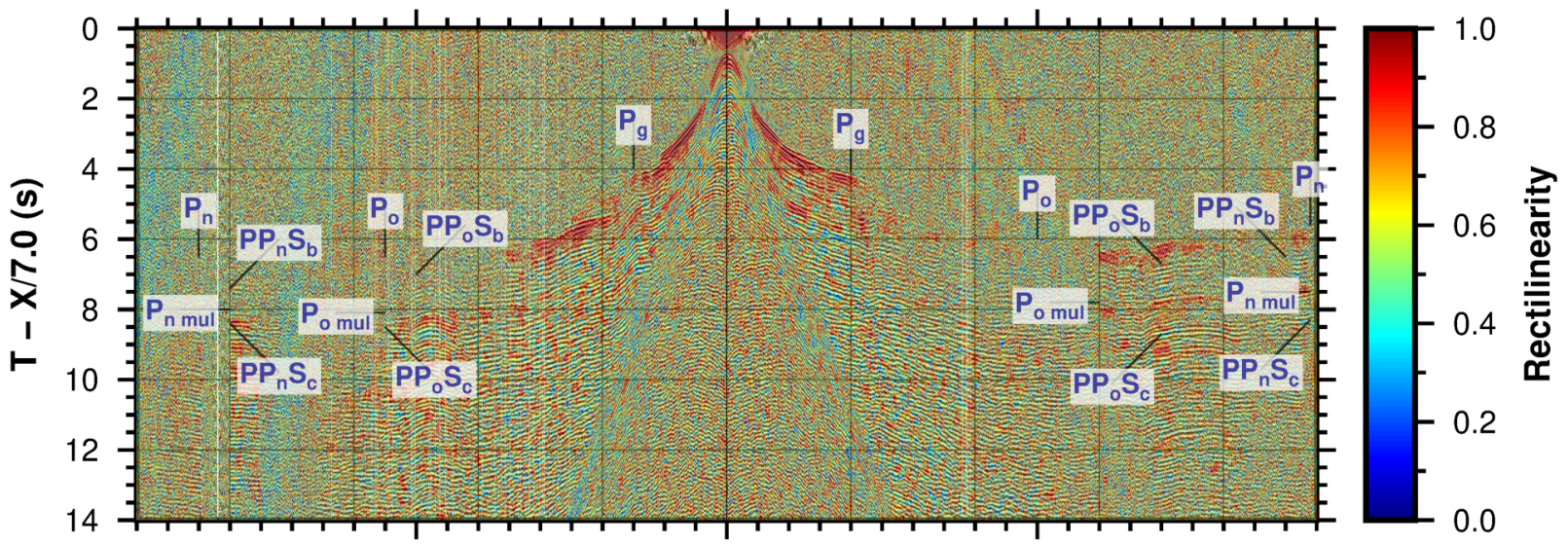

Figure 5.14 Polarisation angles and rectilinearity of the arrivals at OBS 309 overlain on the radial component gather with the travel-time axes reduced at $7.0 \mathrm{~km} / \mathrm{s}$. Observed P and PPS phases are labelled. 
helps to rule out any ambiguities with P-waves and water column multiples. This is very useful in confirming PSS and PPS mode-conversions (Figure 5.12).

\subsection{Identified Mode-converted Phases}

A number mode-converted phases are identified in the radial component of OBS stations between 301 and 328 (Figure 5.2b). At other stations where the horizontal components could be oriented, gathers were noisy and therefore mode-converted waves could not be identified (Figure C.1).

From the identified mode-converted arrivals, the majority are PPS type converted phases (Figures 5.11, 5.12, 5.15, 5.16, 5.17 and 5.18). Some likely PSS phases can be observed, but were not used for further analysis as they could not be confirmed to be real PSS phases.

\subsubsection{PPS Mode-converted Phases}

PPS phases are those where the upgoing P-wave after refraction through a particular layer is converted to an S-wave at an interface. In a travel-time versus offset plot, these phases are parallel to its corresponding direct (unconverted) P-wave arrival and have a time-lag representative of the difference between P-wave velocity and S-wave velocity between the converting interface and the OBS station (Figure 5.19).

PPS phases are identified for all primary refracted phases and the conversions occur at the sediment-basement interface and the top of the oceanic crust. The material on either side of these interfaces are likely to have contrasting physical properties (e.g. density, Pand S-wave speeds), thereby facilitating effective P- to S- and S- to P-conversion (Figure $5.20)$.

The following PPS converted phases are observed in the radial components of the OBS stations.

- $\mathrm{PP}_{\mathrm{g}} \mathrm{S}_{\mathrm{b}}$ - Upgoing $\mathrm{P}_{\mathrm{g}}$ to $\mathrm{S}$ at the base of top sediments

- $\mathrm{PP}_{\mathrm{o}} \mathrm{S}_{\mathrm{b}}$ - Upgoing $\mathrm{P}_{\mathrm{o}}$ to $\mathrm{S}$ at the base of top sediments

- $\mathrm{PP}_{\mathrm{n}} \mathrm{S}_{\mathrm{b}}$ - Upgoing $\mathrm{P}_{\mathrm{n}}$ to $\mathrm{S}$ at the base of top sediments

- $\mathrm{PP}_{\mathrm{o}} \mathrm{S}_{\mathrm{c}}$ - Upgoing $\mathrm{P}_{\mathrm{o}}$ to $\mathrm{S}$ at the top of oceanic crust 

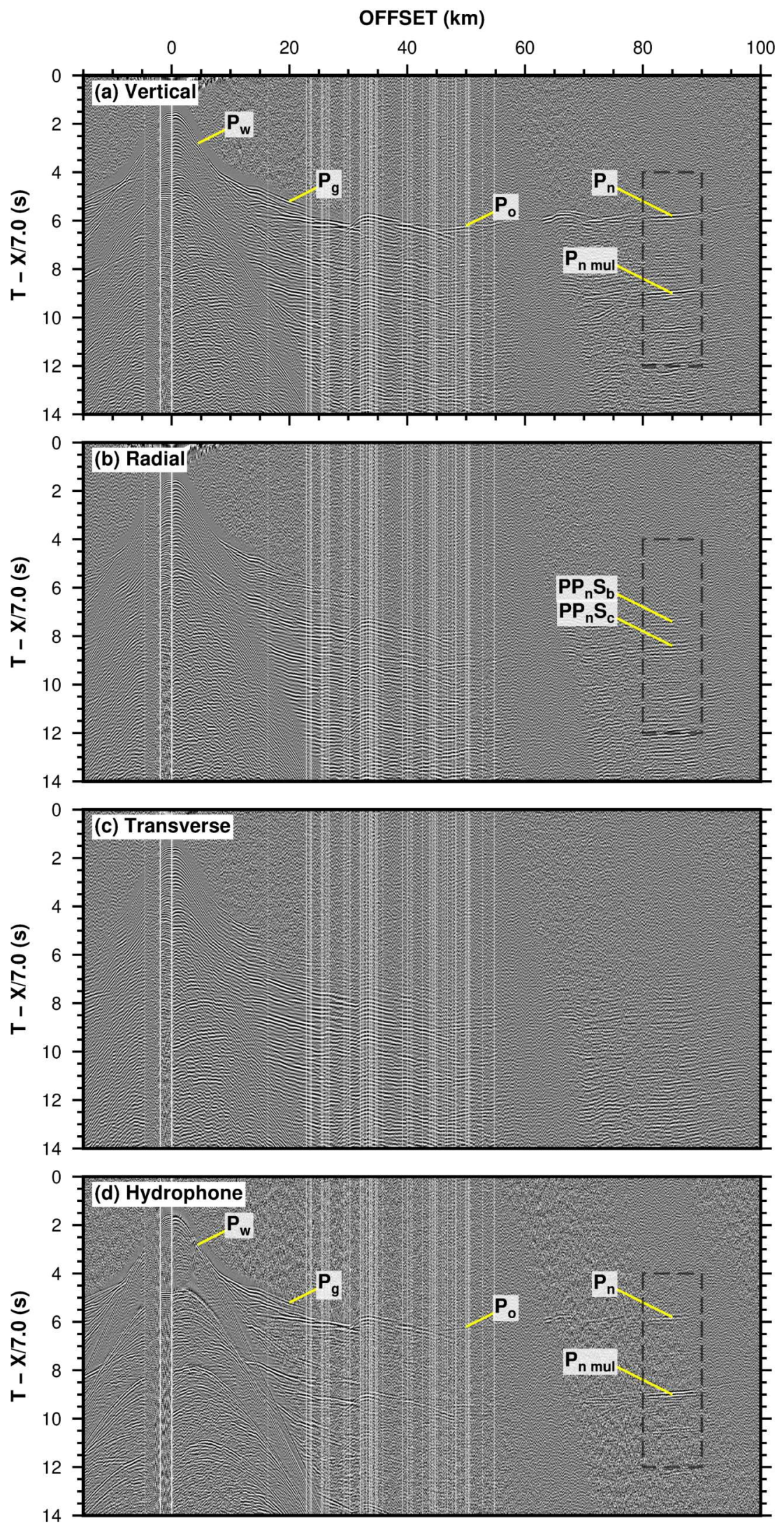

Figure 5.15 Common receiver gathers of (a) vertical, (b) radial, (c) transverse and (d) hydrophone components at OBS 301. Note that the travel-time axes are reduced using a reduction velocity of $7.0 \mathrm{~km} / \mathrm{s}$. Identified seismic phases are labelled. $\mathrm{P}_{\mathrm{g}}$-refraction in the upper plate, $\mathrm{P}_{\mathrm{o}}$-refraction in the oceanic crust, $\mathrm{P}_{\mathrm{n}}$-upper mantle refraction, $\mathrm{P}_{\mathrm{x} \text { mul }}$ indicates the water column multiple of the refractions. $\mathrm{PP}_{\mathrm{x}} \mathrm{S}_{\mathrm{b}}$ are the PPS mode-conversions from the refractions at the base of the top-sediments (subscript " $b$ "). $\mathrm{PP}_{\mathrm{x}} \mathrm{S}_{\mathrm{c}}$ are the PPS mode-conversions from the refractions at the top of the oceanic crust (subscript " $\left.\mathrm{c}^{\prime \prime}\right) . x=\{\mathrm{g}, \mathrm{o}$, n\}. Dashed rectangles indicate sections of each component zoomed in Figure 5.16. 


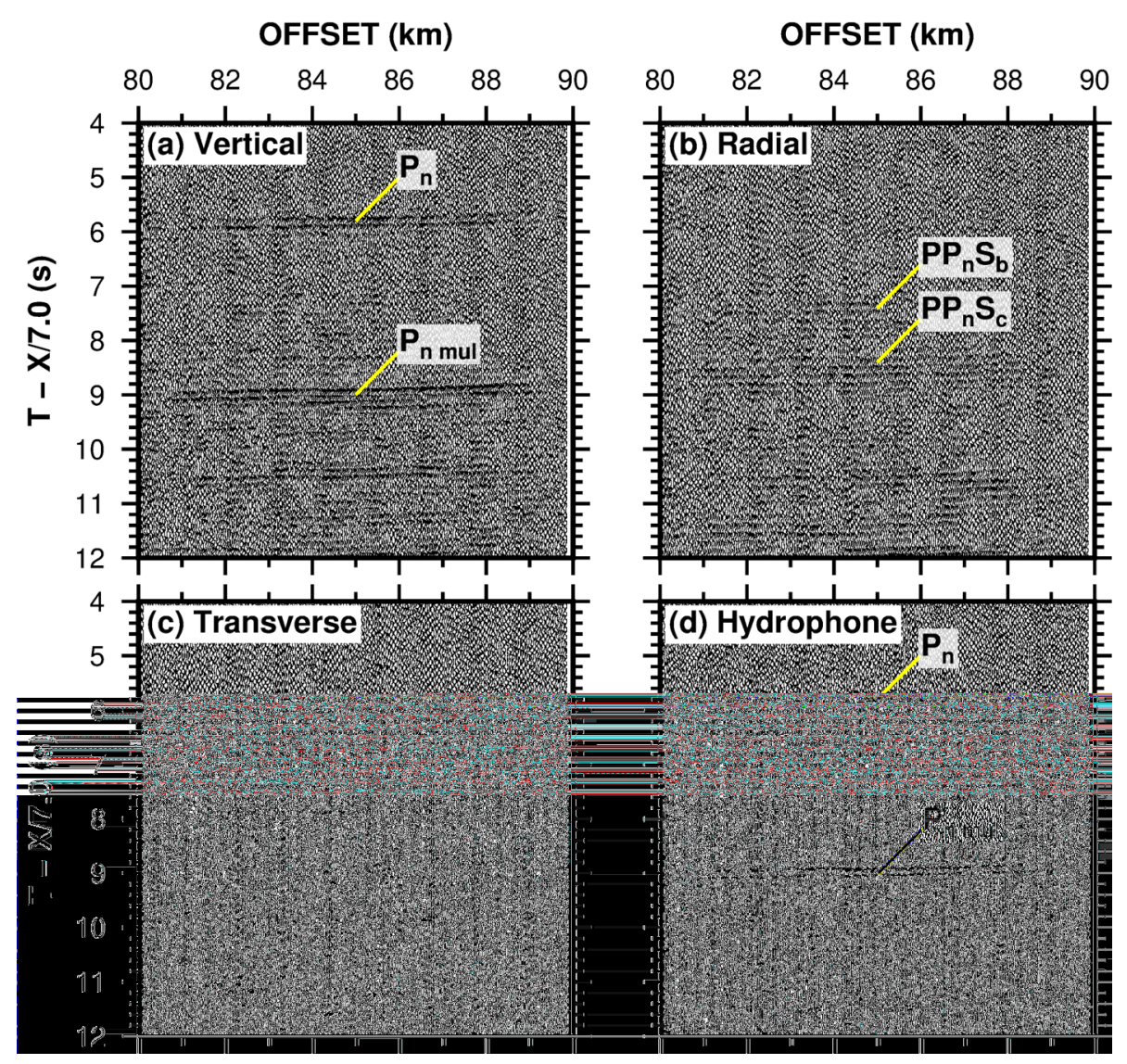

Figure 5.16 Zoomed sections of vertical, radial, transverse and hydrophone components at OBS 301. See Figure 5.15 for a description of the identified phases.

- $\mathrm{PP}_{\mathrm{n}} \mathrm{S}_{\mathrm{c}}-$ Upgoing $\mathrm{P}_{\mathrm{n}}$ to $\mathrm{S}$ at the top of oceanic crust

The first subscript in the phases identified indicates the direct phase being converted to an S phase (e.g. "g" for refraction in the upper crust, "o" for refraction in the oceanic crust and " $\mathrm{n}$ " for upper mantle refraction). The second subscript indicates the interface at which the conversion occurs (e.g. " $b$ " for the base of top sediments, "c" for top of oceanic crust).

\subsection{Average $V_{P} / V_{S}$ Estimation from PPS Mode-converted Phases}

PPS mode-conversions can be modelled using conventional raytracing methods [e.g. Kandilarov et al., 2015; Peacock et al., 2010]. However, raytracing requires a priori estimates of the P-wave velocity and interface structure and is more convenient when good ray coverage from PSS and PSP mode-conversions is available.

PPS mode-conversions can be used to estimate the average $V_{P} / V_{S}$ ratio between the OBS and the conversion interface using the analytical expression of Tsuji et al. [2011] (Equation 5.4), which assumes that both direct P and PPS mode conversions have the 

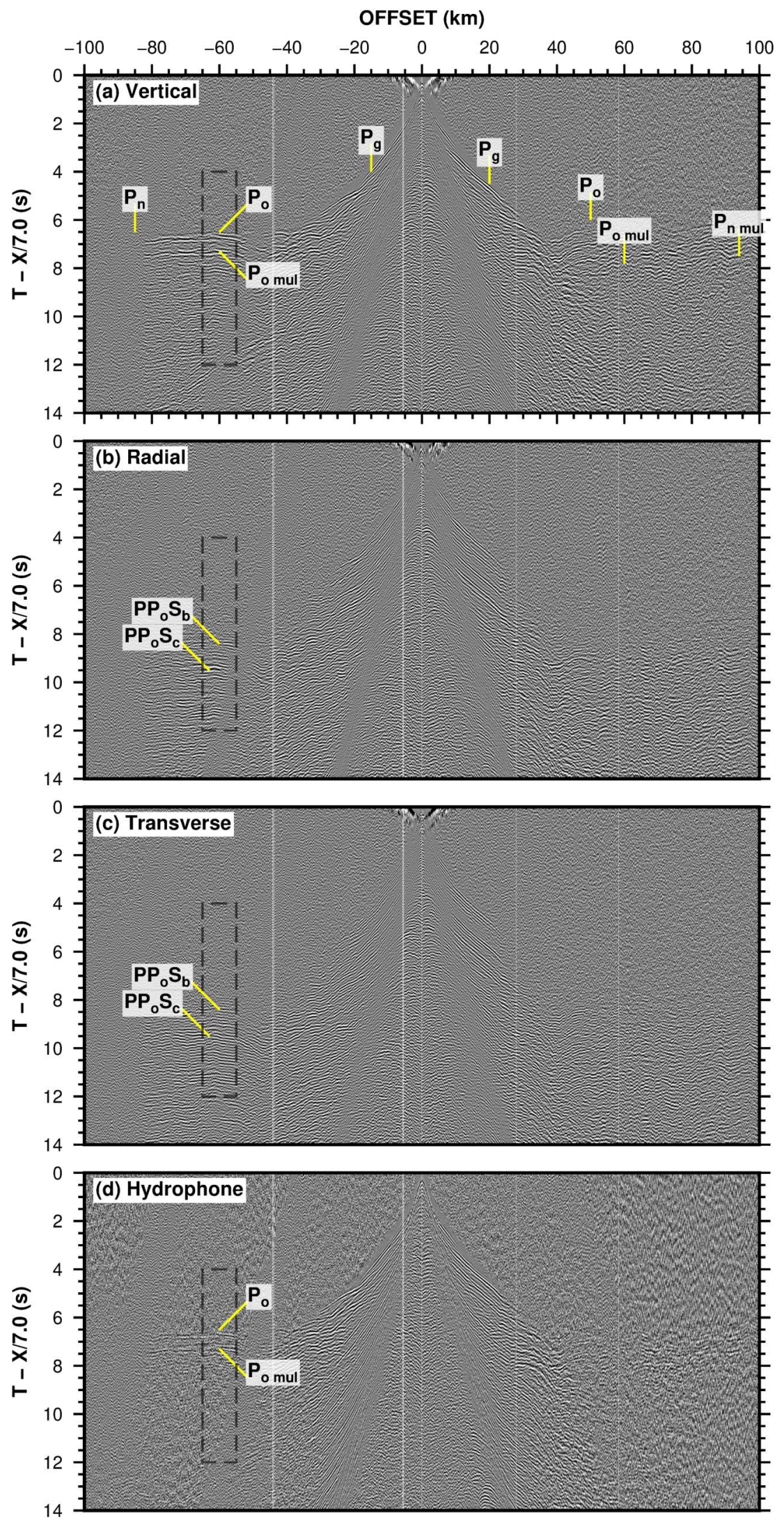

Figure 5.17 Common receiver gathers of (a) vertical, (b) radial, (c) transverse and (d) hydrophone components at OBS 327. Note that the travel-time axes are reduced using a reduction velocity of $7.0 \mathrm{~km} / \mathrm{s}$. Identified seismic phases are labelled. $\mathrm{P}_{\mathrm{g}}$-refraction in the upper plate, $\mathrm{P}_{\mathrm{o}}$-refraction in the oceanic crust, $\mathrm{P}_{\mathrm{n}}$-upper mantle refraction, $\mathrm{P}_{\mathrm{x} \text { mul }}$ indicates the water column multiple of the refractions. $\mathrm{PP}_{\mathrm{x}} \mathrm{S}_{\mathrm{b}}$ are the PPS mode-conversions from the refractions at the base of top sediments (subscript " $b$ "). $\mathrm{PP}_{x} \mathrm{~S}_{\mathrm{c}}$ are the PPS mode-conversions from the refractions at the top of the oceanic crust (subscript "c $\left.c^{\prime \prime}\right) . x=\{g, o, n\}$. Dashed rectangles indicate sections of each component zoomed in Figure 5.18. 


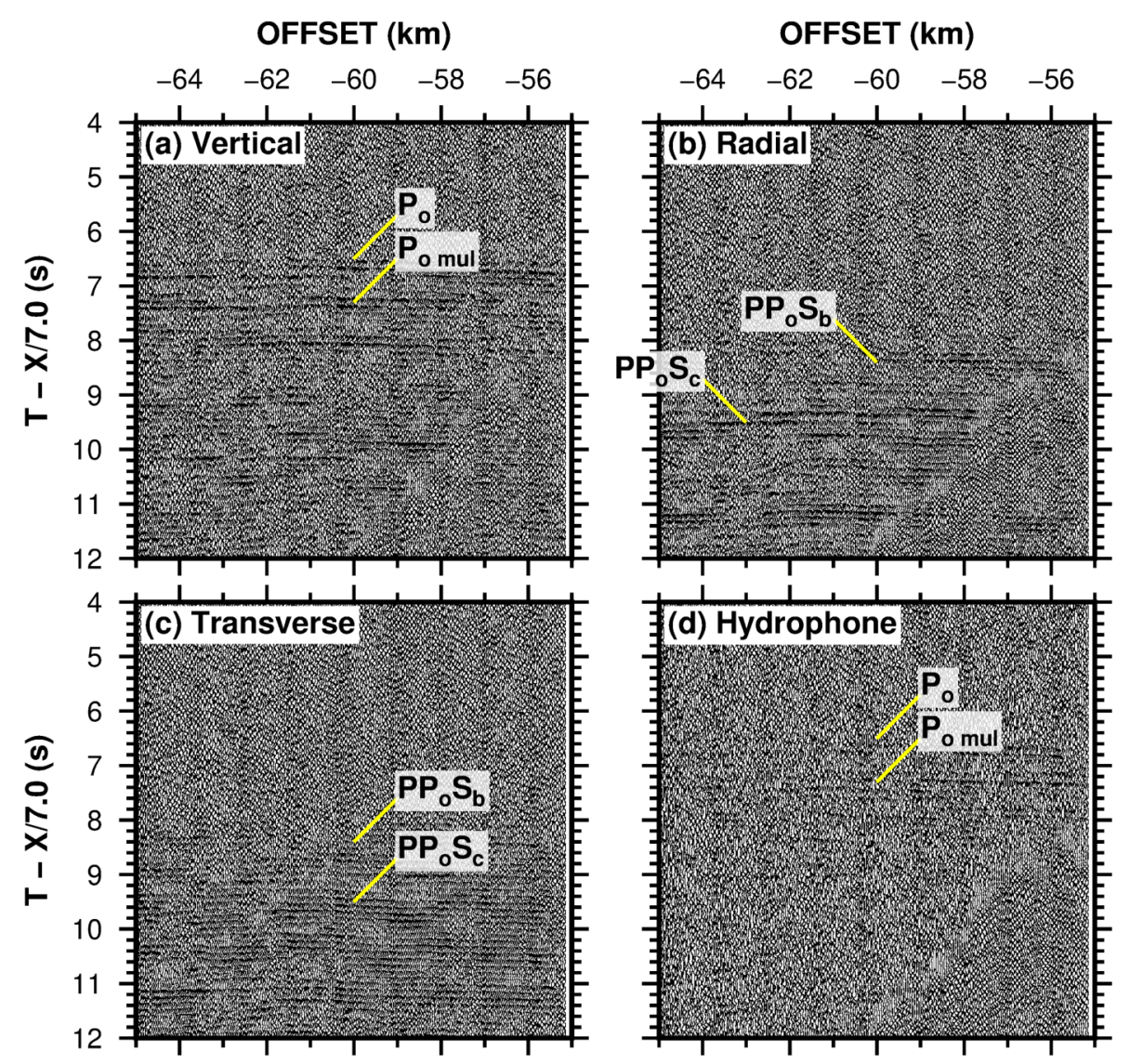

Figure 5.18 Zoomed sections of vertical, radial, transverse and hydrophone components at OBS 327. See Figure 5.17 for a description of the identified phases.

same near-vertical raypaths. The error due to this assumption in the estimated $V_{\mathrm{P}} / \mathrm{V}_{\mathrm{S}}$ is $<2.5 \%$ (assuming the crustal structure in Figure $5.20 \mathrm{~b}$ ) and thus can be considered negligible.

$$
\frac{V_{\mathrm{P}}}{V_{\mathrm{S}}}=\frac{2 \Delta T+\left(T_{\mathrm{P}}-T_{\mathrm{Psf}}\right)}{\left(T_{\mathrm{P}}-T_{\mathrm{Psf}}\right)}
$$

Here, $\Delta \mathrm{T}$ is the travel-time lag of the PPS phase with the P-phase. $T_{P}$ is the zero-offset travel-time of the reflected wave from the converted horizon and $\mathrm{T}_{\mathrm{Psf}}$ is the zero offset two-way travel-time of the seafloor reflection in the coincident multichannel seismic reflection image.

\subsubsection{Calculating $\Delta \mathrm{T}$}

The travel-time lag $(\Delta \mathrm{T})$ is the difference between the travel-times of the PPS phase and its corresponding P phase (Figure 5.19b). For this, the travel-times of the P-waves and the PPS phases are picked. However, the pick error of the travel-times of the converted 


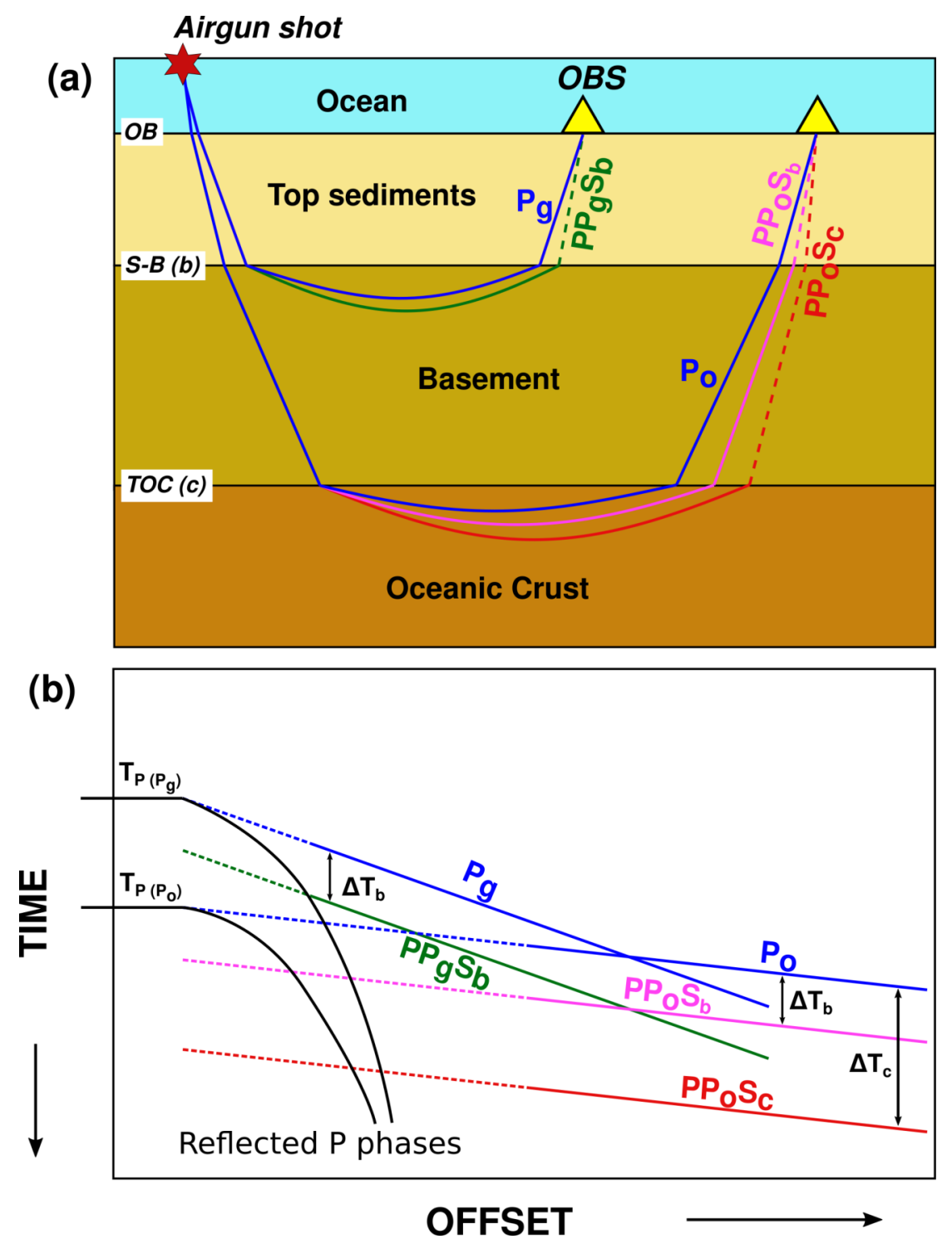

Figure 5.19 (a) Subsurface layers and the possible PPS converted arrivals and their conversion interfaces. (b) Travel-time versus offset plot for the observed direct, first arriving refracted P-wave arrivals and subsequent PPS arrivals.

phases could be as high as $\sim 0.15 \mathrm{sec}$, which would introduce higher uncertainties in the estimated average $V_{P} / V_{S}$ ratios (Equation 5.4).

Therefore, to clearly identify and pick the onset of the PPS phase, a one-second window centred around the P-phase arrival of the vertical component is cross-correlated with a one-second window centred around the PPS phase in the radial component. This is applied for all the traces in the vertical and radial components where both $\mathrm{P}$ and PPS phases are identified. Travel-time lags are calculated when the cross-correlation coefficient is greater than 0.75 and the corresponding time-shift is within the highest pick uncertainty of $\sim 0.15 \mathrm{sec}$ (Figures $5.21,5.22$ ). The mean travel-time lag is taken from all the cross-correlations for a particular PPS conversion. 
(a)

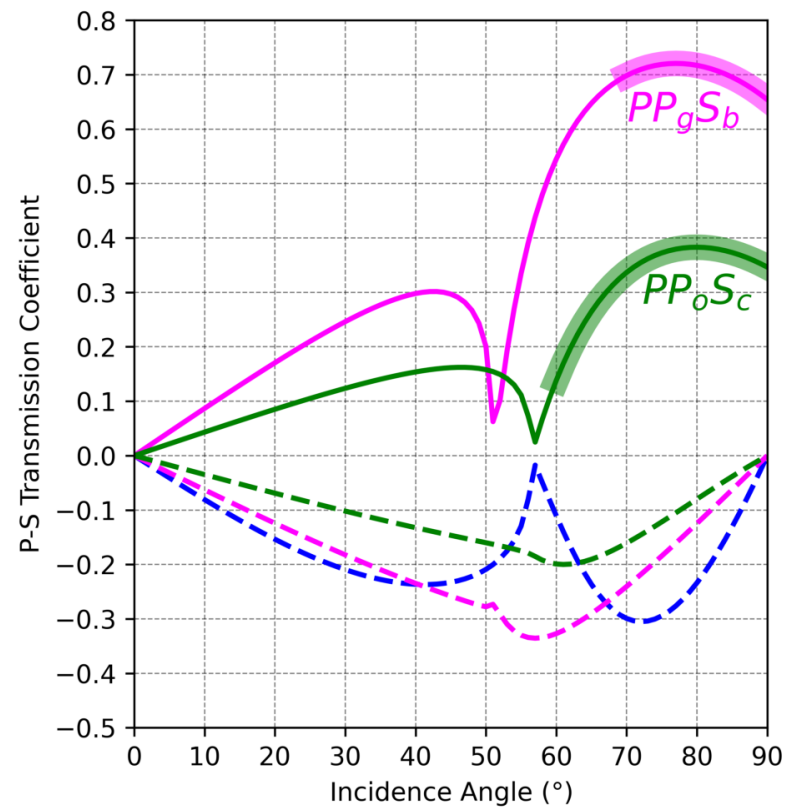

(b)

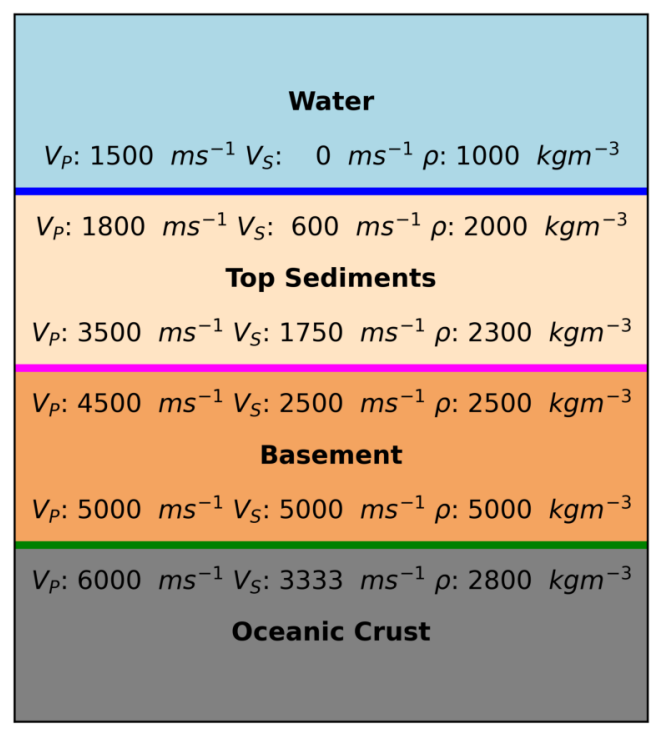

Figure 5.20 (a) P- to S- transmission coefficients calculated from Zoeppritz equations for upgoing (solid lines) and downgoing (dashed lines) incident plane P-waves with a unit magnitude at interfaces in (b). Shaded sections in the solid curves indicate the range of incidence angles for P- to S- conversion of refracted wave in the layer beneath that interface.
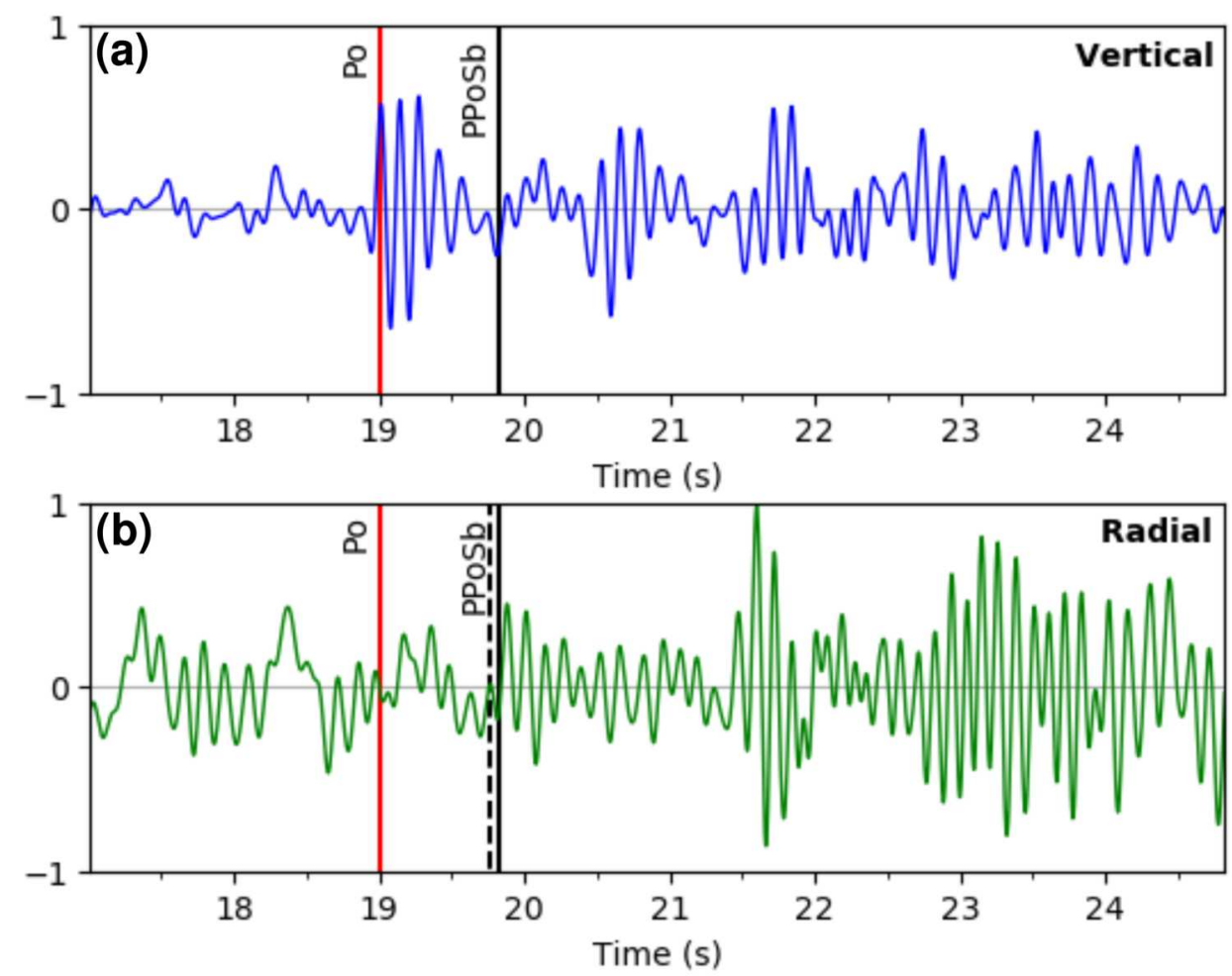

Figure 5.21 (a) Vertical and (b) radial component traces showing the travel-time lag between the first arriving $\mathrm{P}_{\mathrm{o}}$ phase and the $\mathrm{PP}_{\mathrm{o}} \mathrm{S}_{\mathrm{b}}$ converted phase. Continuous black line indicates the travel-time pick of the PPS phase. Dashed black line indicates the travel-time pick arrival of the PPS phase from crosscorrelation. 

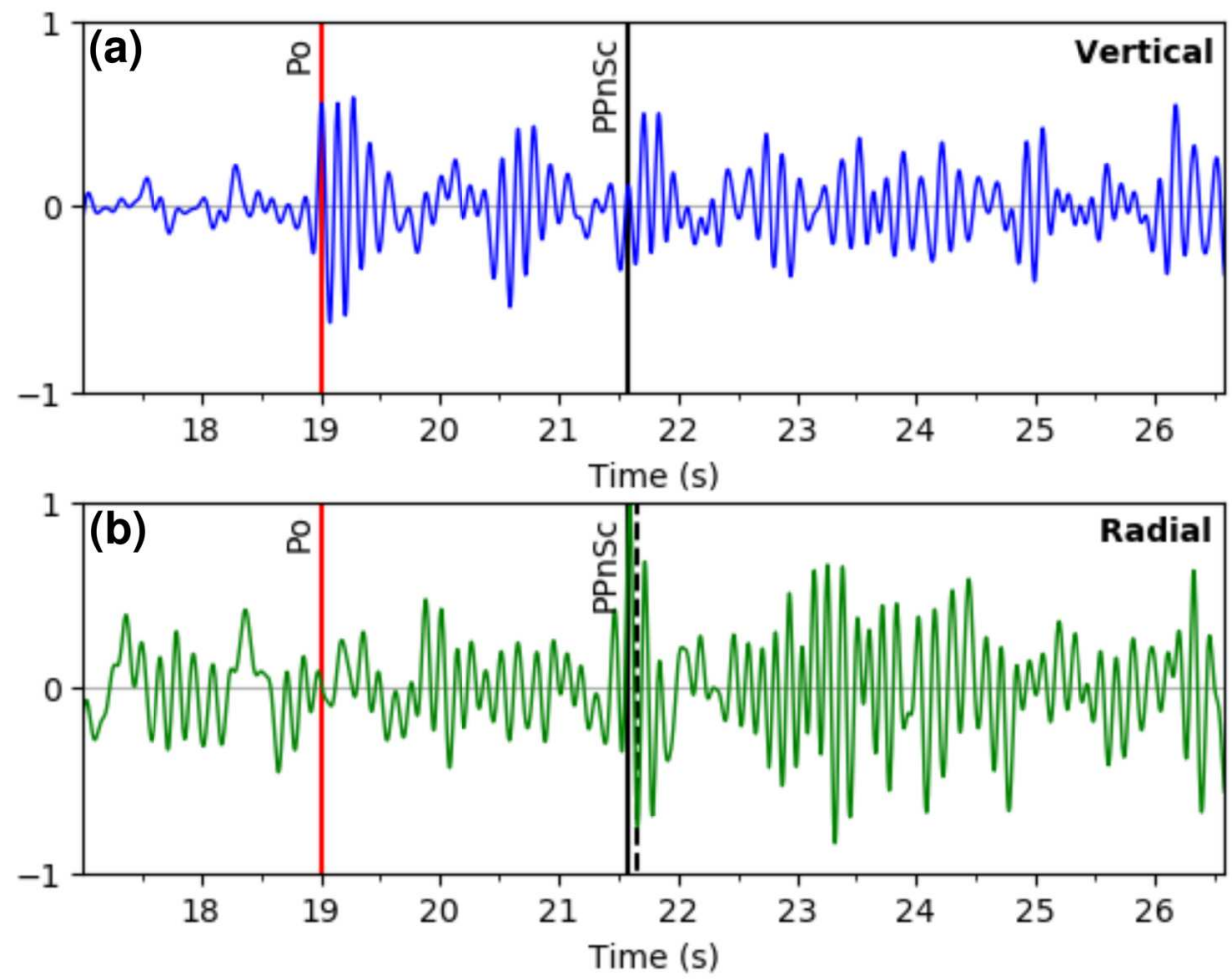

Figure 5.22 (a) Vertical and (b) radial component traces showing the travel-time lag between the first arriving $\mathrm{P}_{\mathrm{o}}$ phase and the $\mathrm{PP}_{\mathrm{o}} \mathrm{S}_{\mathrm{c}}$ converted phase. Continuous black line indicates the travel-time pick of the PPS phase. Dashed black line indicates the travel-time pick arrival of the PPS phase from crosscorrelation.

\subsubsection{Calculating $\mathrm{T}_{\mathrm{P}}$ and $\mathrm{T}_{\mathrm{Psf}}$}

Equation 5.4 requires two other parameters to calculate average $V_{P} / V_{S}, T_{P}$ and $T_{\text {Psf }}$. These values are usually obtained from multichannel seismic reflection data by comparing with the radial component OBS gather [e.g. Tsuji et al., 2011].

However, in this study, it was not possible to obtain $\mathrm{T}_{\mathrm{P}}$ from MCS reflection data for the following reasons. It is difficult to correlate a particular horizon in the MCS reflection data with a refracted phase when the phase is observed at far offsets and is non-linear due to the presence of seafloor topography. Sometimes deeper horizons, where conversions occur, are not observed in the MCS reflection data.

For $\mathrm{PP}_{\mathrm{x}} \mathrm{S}_{\mathrm{b}}$ phases $(\mathrm{x}=\mathrm{g}, \mathrm{o}, \mathrm{n})$, where the conversion is assumed to occur at the base of top sediments, $\mathrm{T}_{\mathrm{P}}$ was taken from the most prominent horizon beneath the OBS station (Figure 5.23). To calculate $T_{P}$ for the $\mathrm{PP}_{\mathrm{x}} \mathrm{S}_{\mathrm{c}}$ phases $(\mathrm{x}=\mathrm{O}, \mathrm{n})$, we assume that the top of the oceanic crust, i.e. the converting interface, could be located at 0,1 and $2 \mathrm{~km}$ deeper than the plate interface of Williams et al. [2013]. This assumption is based on the fact that such depths are observed to the top of the subducting crust from the plate 


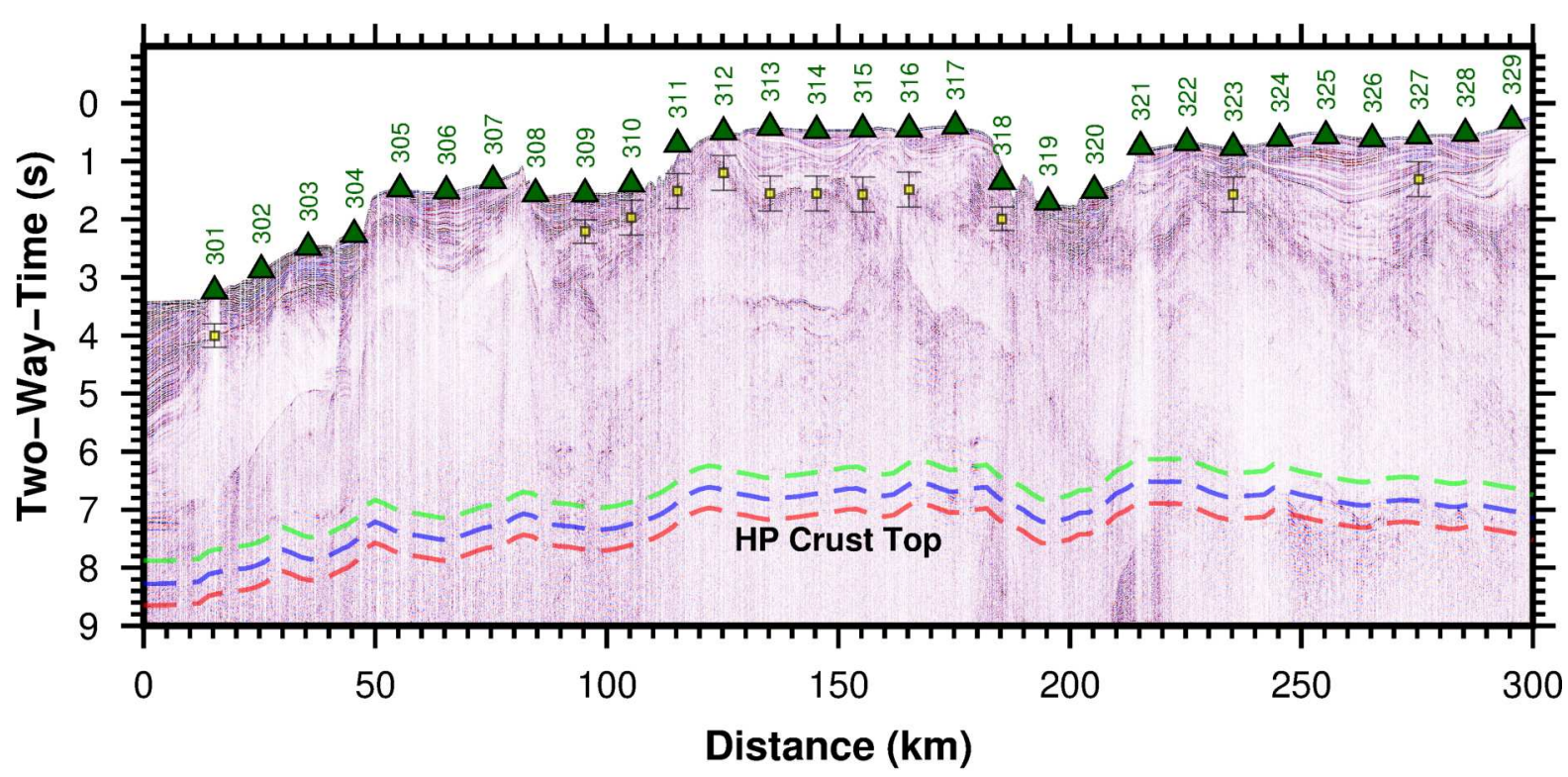

Figure 5.23 Multichannel seismic reflection image along SHIRE03 OBS profile. Yellow squares indicate the two-way travel-time to the sediment-basement interface, with approximate errors. Red, blue and green dashed lines indicate two-way travel-times of the horizons at depths of $0 \mathrm{~km}, 1 \mathrm{~km}$ and $2 \mathrm{~km}$, respectively from the plate interface model of Williams et al. [2013] calculated from the $V_{P}$ model.

interface in the controlled-source seismic studies in the region [e.g. Bland et al., 2015; Henrys et al., 2013]. The two-way travel-time to these interfaces beneath each OBS are calculated (Figure 5.23) using the $\mathrm{V}_{\mathrm{P}}$ model derived from the tomographic inversion of first arrivals along the SHIRE03 OBS profile [Bassett et al., 2018].

Two-way travel-time of the seafloor reflection $\left(\mathrm{T}_{\mathrm{Psf}}\right)$, at each OBS location was determined from the MCS reflection section (Figure 5.23), assuming that deviation of the OBS station from the MCS line is negligible.

Uncertainties in $\mathrm{V}_{\mathrm{P}} / \mathrm{V}_{\mathrm{S}}$ are estimated using error propagation based on Equation 5.4, in which the error in $\Delta \mathrm{T}$ is the standard deviation of the travel-time delays calculated for all the traces. Error in $\mathrm{T}_{\mathrm{Psf}}$ is $0.1 \mathrm{sec}$, which is the pick uncertainty of the ocean bottom in the MCS reflection data (Figure 5.23). Error in $T_{P}$ for the base of the top sediments $\left(\mathrm{PP}_{\mathrm{x}} \mathrm{S}_{\mathrm{b}}\right.$ phases) estimated by visual inspection of the MCS reflection data (Figure 5.23). Error in $\mathrm{T}_{\mathrm{P}}$ for the top of the Hikurangi Plateau crust ( $\mathrm{PP}_{\mathrm{x}} \mathrm{S}_{\mathrm{c}}$ phases) is assumed to be $0.2 \mathrm{sec}$ to account for an uncertainty of $\pm 1 \mathrm{~km}$ in the depth to the top of the subducting crust.

Using this approach, the average $\mathrm{V}_{\mathrm{P}} / \mathrm{V}_{\mathrm{S}}$ ratio for: (1) top sediments and; (2) entire forearc sedimentary section overlying the top of the subducting Hikurangi Plateau crust were calculated for the OBS stations from 301 to 328 (Figure 5.24). 


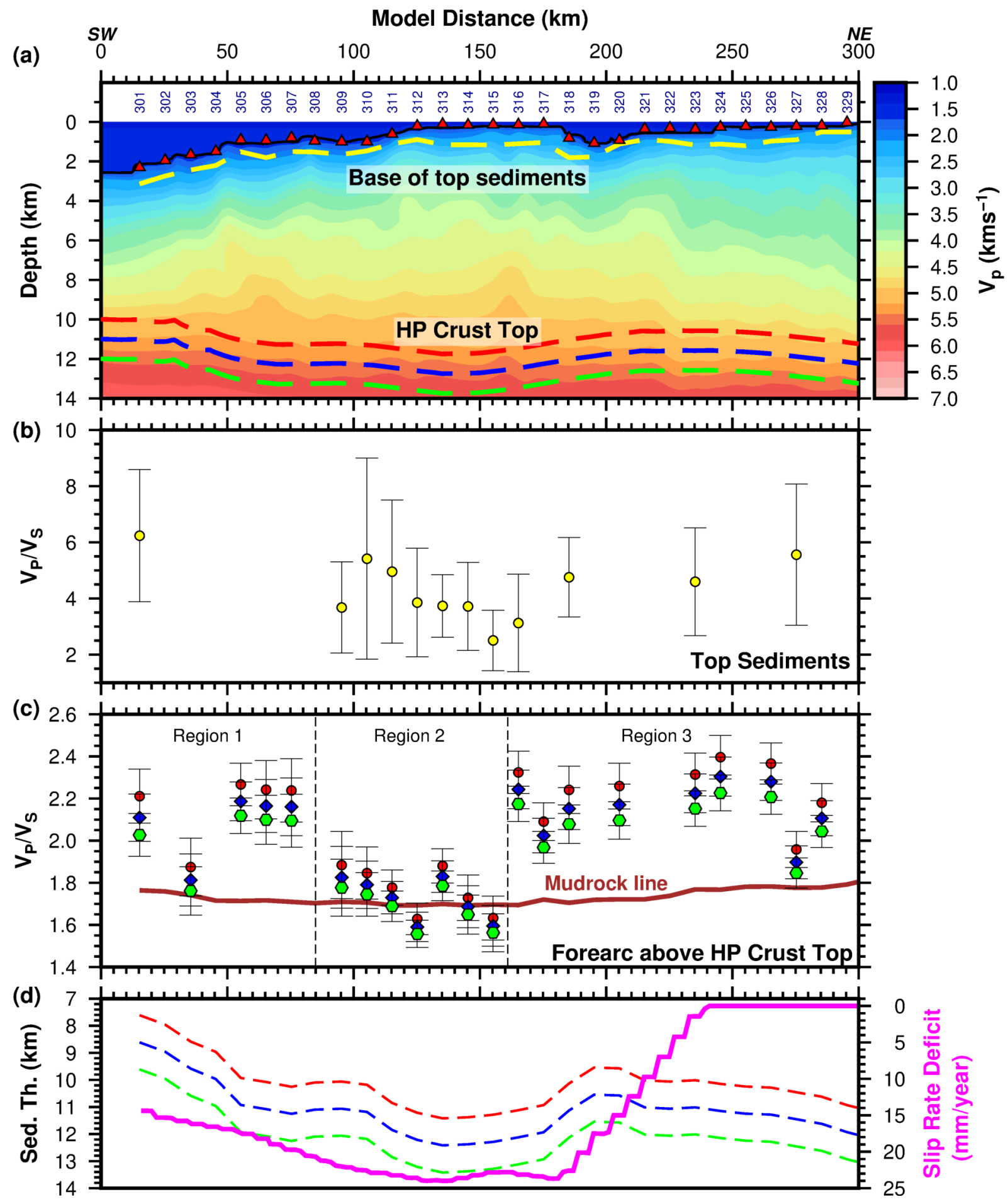

Figure 5.24 (a) P-wave velocity model along Hikurangi margin forearc [Bassett et al., 2018]. Yellow dashed line indicates the base of the top layer of sediments. Red, blue and green dashed lines indicate the depths of $0 \mathrm{~km}, 1 \mathrm{~km}$ and $2 \mathrm{~km}$, respectively from the plate interface model of Williams et al. [2013]. (b) Average $V_{P} / V_{S}$ ratio for the top layer of sediments beneath the seafloor. (c) Average $V_{P} / V_{S}$ ratio for the sediments and rocks above the top of the oceanic crust for different depths of the top of the oceanic crust from the plate interface model of Williams et al. [2013] (red=0 km, blue=1 km and green=2 km). $V_{P} / V_{S}$ ratio for "mudrock" [Castagna et al., 1985] is calculated using the average P-wave velocity between ocean bottom and the top of the Hikurangi Plateau crust in (a). (d) Variation of sedimentary thickness above the subducting crust (dashed lines colour-coded similar to (a)) and slip-rate deficit (pink) [Wallace et al., $2012 b$ ] along the model. 


\subsection{Shear-wave Splitting}

Shear-wave splitting is widely used to assess seismic anisotropy in the Earth. It is based on the observation that when a shear-wave enters an anisotropic medium, it splits into two separate waves, one which travels faster and the other slower [Savage, 1999; Stein and Wysession, 2003]. The faster wave is polarised parallel to the fast direction and leads the orthogonal slow wave. Shear-wave splitting can be used to calculate the fast polarisation direction $(\phi)$ and the delay time $(d t)$, which can then be used to infer tectonic processes, such as deformation or state of stresses in the region. In addition to this, shear-wave splitting can provide insights as to whether the determined $\mathrm{V}_{\mathrm{P}} / \mathrm{V}_{\mathrm{S}}$ ratios are influenced by anisotropy.

Shear-wave splitting has been widely used with SKS phases in assessing the anisotropic nature of the upper mantle [Gledhill and Gubbins, 1996; Savage, 1999; Silver and Chan, 1988]. Crustal anisotropy using local earthquakes has also been assessed in a variety of tectonic settings [e.g. Crampin, 1990; Graham et al., 2020; Savage et al., 2010; Zal et al., 2020]. Shear-wave splitting has also been used to measure anisotropy with mode-converted phases in controlled-source seismic data, specially PPS phases [e.g. Kandilarov et al., 2015; Peacock et al., 2010; Pulford et al., 2003; Tsuji et al., 2011]. However, most of these studies use hodogram analyses to estimate $\phi$ and $d t$.

In this study, we take a different approach to calculate $\phi$ and $d t$ using the observed PPS phases. We employ the fully automated MFAST package [Savage et al., 2010]. It implements a multiple window and multiple filter splitting technique to calculate $\phi$ and $d t$ (Figure 5.25). To increase the signal-to-noise ratio, $10 \mathrm{sec}$ long stacked radial and transverse traces are produced by summing the traces using the PPS arrival time as the reference point for the summation. Multiple bandpass filters between 1-12 Hz, 1-14 Hz, 1-16 Hz were employed.

MFAST calculates splitting parameters only when the signal-to-noise ratio is greater than a given threshold ( 2 in this case), and calculates splitting parameters over 75 windows with different start and end times. The best-fit from all the windows is determined from a cluster analysis and grading results based on the signal-to-noise ratio, uncertainty, distinctiveness of the cluster, and whether the measured parameters are a null measurement [Savage et al., 2010] (Figure 5.25). Adhering to these criteria, $\phi$ 
and $d t$ could be calculated only at a few stations (Figure 5.27).

\subsection{Results and Discussion}

\subsubsection{Average $\mathrm{V}_{\mathrm{P}} / \mathrm{V}_{\mathrm{S}}$ Ratio from PPS Phases}

\subsubsection{Top Sediments of the Hikurangi Forearc}

Average $\mathrm{V}_{\mathrm{P}} / \mathrm{V}_{\mathrm{S}}$ ratios ranging from $\sim 2.5 \pm 1.0$ up to $\sim 6.0 \pm 2.5$ are estimated for the seafloor sediments (Figure $5.24 \mathrm{~b}$ ). Uncertainties in the average $V_{P} / V_{S}$ ratios are fairly high as a higher error in the determination of $\mathrm{T}_{\mathrm{P}}$ is incorporated in to the calculation. The thickness of these sediments is variable between the seafloor and the horizon showing a clear impedance contrast beneath the seafloor in the MCS section (Figures 5.23 and 5.24a). These high $\mathrm{V}_{\mathrm{P}} / \mathrm{V}_{\mathrm{S}}$ ratios indicate that these sediments are unconsolidated and water-saturated. Based on the empirical relationships of Castagna et al. [1985] between porosity and clay content with $\mathrm{V}_{\mathrm{P}} / \mathrm{V}_{\mathrm{S}}$ ratio, these values are consistent with sediments whose porosity can vary from $\sim 20 \%$ up to $\sim 55 \%$ for clay contents varying from $0 \%$ to $100 \%$ (Figure 5.26 ).

Similarly, high average $\mathrm{V}_{\mathrm{P}} / \mathrm{V}_{\mathrm{S}}$ ratios of $\sim 5-10$ are reported for the sediments in Cascadia basin on top of the incoming Juan de Fuca Plate [Zhu et al., 2020]. Tsuji et al. [2011] also reports average $\mathrm{V}_{\mathrm{P}} / \mathrm{V}_{\mathrm{S}}$ ratios of 2.5-3.5 fo the top sediments at Nankai margin.

\subsubsection{Sedimentary Section above the Subducting Hikurangi Plateau Crust}

Average $V_{\mathrm{P}} / \mathrm{V}_{\mathrm{S}}$ ratios of the sedimentary section above the top of the subducting Hikurangi Plateau crust range from from $\sim 1.60 \pm 0.06$ to $\sim 2.4 \pm 0.09$ with local variations (Figure 5.24c), when the converting interface (i.e. top of the Hikurangi Plateau crust) is at the plate interface of Williams et al. [2013]. When the depth to the converting interface increases by $2 \mathrm{~km}$, the average $V_{P} / V_{S}$ ratios decrease by a maximum of $\sim 0.2$.

The following discussion is based on the average $V_{P} / V_{S}$ ratios calculated with the converting interface at a depth of $2 \mathrm{~km}$ from the plate interface model of Williams et al. [2013] as similar depths are reported for the top of the Hikurangi Plateau crust from the plate interface in the MCS reflection images [e.g. Bland et al., 2015].

In between OBS 301 and 307 (Region 1 in Figure 5.24c), average $V_{P} / V_{S}$ varies between $\sim 1.75 \pm 0.11$ and $2.10 \pm 0.08$ with an average of 2.00. It reflects the presence high-porosity 
(a)

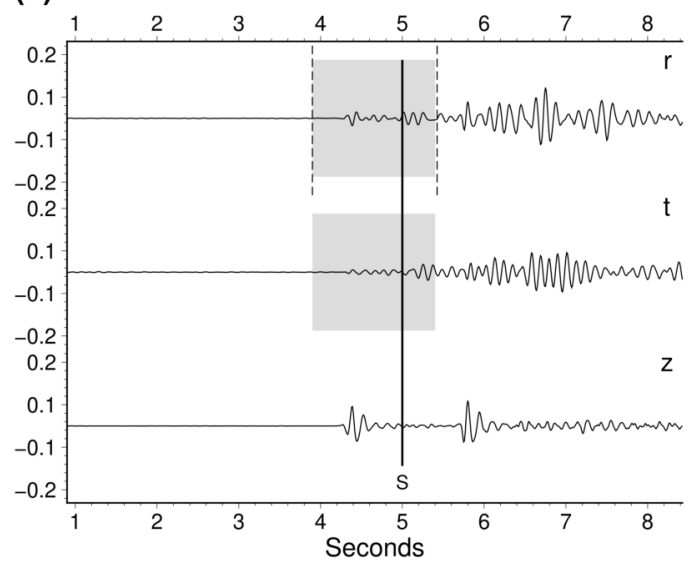

(c)

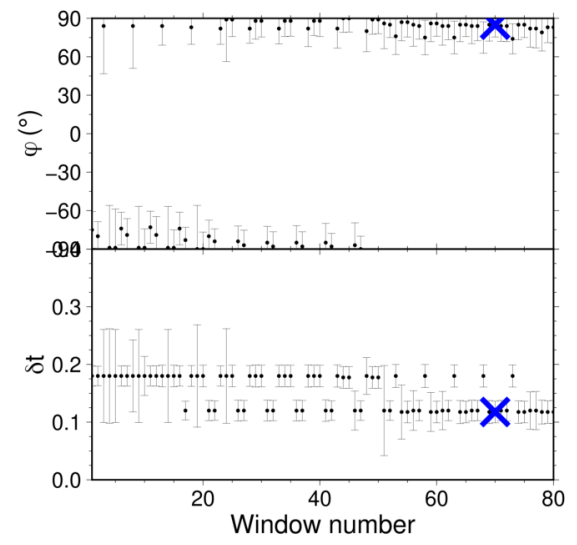

(e)
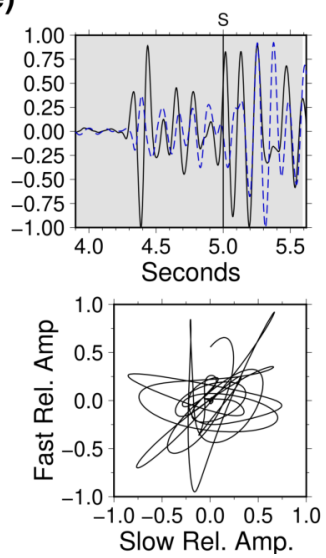
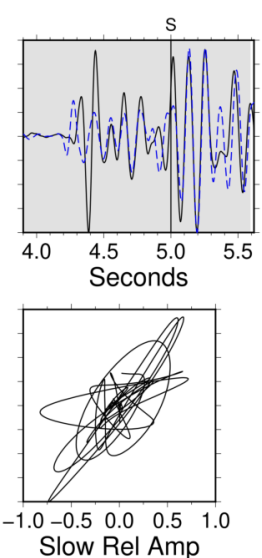

(b)

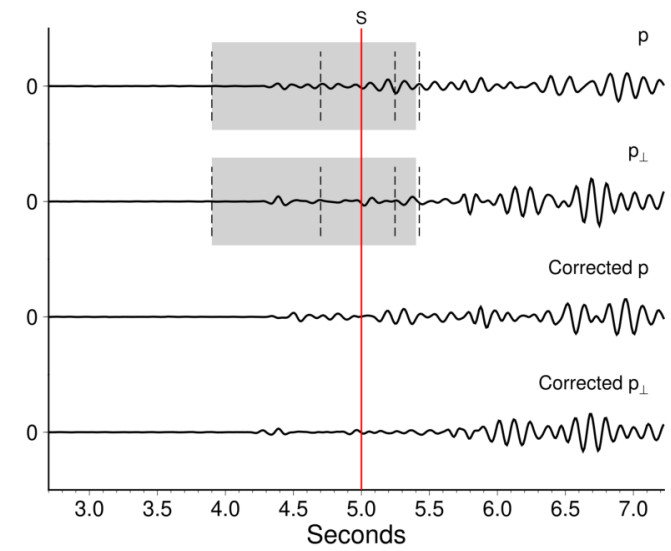

(d)

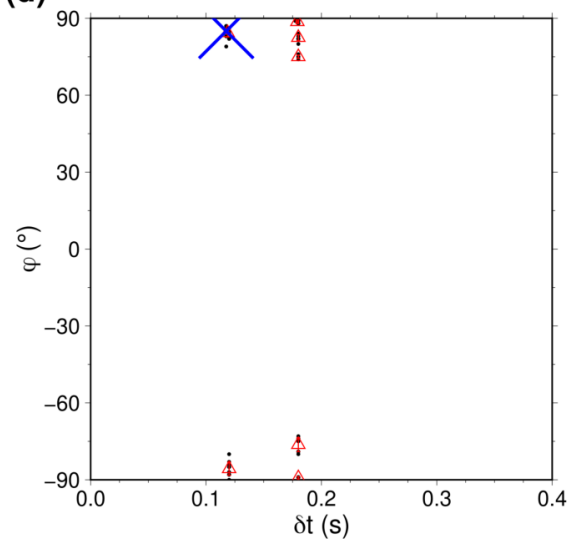

(f)

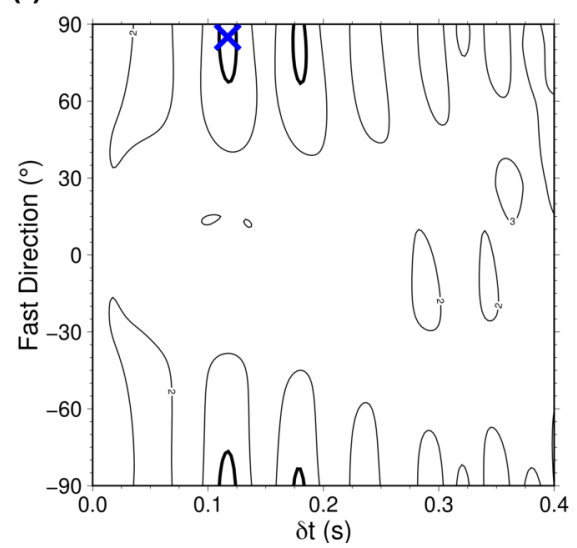

Figure 5.25 Shear-wave splitting result at OBS 309 from MFAST. (a) Radial (r), transverse ( $t$ ) and vertical (z) components bandpass filtered at 1-16 Hz. Vertical black line is the S-wave arrival. Dashed lines indicate the minimum start and maximum end times. Gray shaded region is the window of the final measurement. (b) Normalised radial and transverse waveforms and radial and transverse waveforms corrected for splitting. (c) $\phi$ and $d t$ result for each window. Cross indicates the final result. (d) Clustering of best results in $p h i, d t$ space. Cross indicates the final result. (e) Top plots indicate the match between slow and fast waveforms before and after splitting. Bottom plots show the particle motion before and after splitting. (f) Clustering of best measurement with contour of the smallest eigenvalue of the covariance matrix for final result. Cross mark indicates the final result. 
and potentially water-saturated material (Figure 5.24c). This region is closer to the deformation front (Figure 5.2b) and the high $\mathrm{V}_{\mathrm{P}} / \mathrm{V}_{\mathrm{S}}$ ratios may be due to the lower thickness of the sedimentary section overlying the subducting crust, resulting in less compaction (Figure 5.24d).

In between OBS 309 and 315 (Region 2 in Figure 5.24c), average $V_{P} / V_{S}$ is comparatively low $(\sim 1.55 \pm 0.08$ to $1.78 \pm 0.12)$ with an average $\mathrm{V}_{\mathrm{P}} / \mathrm{V}_{\mathrm{S}}$ of 1.67 . These estimates fall close to the empirically derived $V_{P} / V_{S}$ ratio for "mudrock", which is defined as a clastic silicate rock comprised of clay- or silt-sized particles [Castagna et al., 1985] (Figure 5.24c). These $\mathrm{V}_{\mathrm{P}} / \mathrm{V}_{\mathrm{S}}$ ratios are also consistent with $\mathrm{SiO}_{2}$ rich, water-saturated, consolidated sediments with clay contents ranging from $0 \%$ to $30 \%$ and porosity $<30 \%$, which are subjected to higher differential stresses [Lee, 2003]. The low porosity, low clay content (high $\mathrm{SiO}_{2}$ content) may highlight the presence of Cretaceous greywackes, possibly an offshore extension of the Pahau Torlesse Terrane found onshore in the southeastern North Island [Mortimer, 2004]. This is further corroborated as the $\mathrm{V}_{\mathrm{P}} / \mathrm{V}_{\mathrm{S}}$ estimates from this study are comparable with those of the Pahau Terrane $\left(\mathrm{V}_{\mathrm{P}} / \mathrm{V}_{\mathrm{S}}<1.80\right)$ as well as the Rakaia Terrane west of Pahau $\left(\mathrm{V}_{\mathrm{P}} / \mathrm{V}_{\mathrm{S}} \sim 1.65-1.80\right)$ [Eberhart-Phillips et al., 2005], which are found to be lithologically similar [Mortimer, 2004]. However, the Rakaia Terrane is thought to be impermeable and therefore is suggested to be the cause of deeper down-dip plate interface locking [Reyners and Eberhart-Phillips, 2009]. These compacted and lithified sandstones have characteristic $\mathrm{V}_{\mathrm{P}} / \mathrm{V}_{\mathrm{S}}$ ratios similar to metagreywackes (1.711-1.748) at 200-1000 MPa [Christensen, 1996], which further confirms the low-porosity. In addition, the higher thickness of the sedimentary section in this region (Figure 5.24d) may increase the degree of compaction, which may also contribute to the lower $V_{\mathrm{P}} / \mathrm{V}_{\mathrm{S}}$ ratios. This region also shows a highly reflective lower crust of the forearc capped by a high amplitude laterally contiguous reflector (Figure 5.23). This highly reflective forearc region has also been interpreted as the Pahau Torlesse Terrane [Mountjoy and Barnes, 2011].

From OBS 315 to $316, \mathrm{~V}_{\mathrm{P}} / \mathrm{V}_{\mathrm{S}}$ ratio increases rapidly from $\sim 1.56 \pm 0.09$ to $\sim 2.17 \pm 0.08$. It varies between $\sim 1.84 \pm 0.07$ and $\sim 2.22 \pm 0.08$ between OBS 316 and 328 (Region 3 in Figure $5.24 \mathrm{c}$ ) with an average of $\sim 2.08$. In addition, material in this region has lower sedimentary thicknesses (Figure 5.24d) and reduced P-wave velocities, indicated by the 
down-warping of velocity contours in the P-wave velocity model (Figure 5.24a). The high $\mathrm{V}_{\mathrm{P}} / \mathrm{V}_{\mathrm{S}}$ ratios cannot be explained by the mudrock line (Figure 5.24c), and clearly reflect the presence of compositionally different sediments to those in the immediate southwest. If porosity alone is used to explain the high $\mathrm{V}_{\mathrm{P}} / \mathrm{V}_{\mathrm{S}}$ ratios here, it corresponds to a porosity of $\sim 40-45 \%$. However, with increasing clay-content, the porosity required to explain the high $\mathrm{V}_{\mathrm{P}} / \mathrm{V}_{\mathrm{S}}$ ratios decreases to $\sim 5 \%$ at $100 \%$ clay content (Figure 5.26 ). On the other hand, according to Lee [2003], the high $\mathrm{V}_{\mathrm{P}} / \mathrm{V}_{\mathrm{S}}$ ratios are also consistent with water-saturated, unconsolidated sediments with $0 \%-30 \%$ clay content and $20-40 \%$ porosity and subjected to lower differential stresses.

\subsubsection{Anisotropy and $\mathrm{V}_{\mathrm{P}} / \mathrm{V}_{\mathrm{S}}$ Ratios}

Shear-wave splitting results using PPS phases converted at the base of the top layer of sediments and the top of the oceanic crust of the Hikurangi Plateau indicate similar results (Figure 5.27). Fast shear-wave polarisation azimuths are nearly margin-perpendicular except at OBS 309, which is subparallel to the margin (Figure 5.27a). The margin-perpendicular fast azimuths may indicate the stress state within the forearc, potentially due to plate convergence indicated by similarly oriented maximum horizontal compressive stress $\left(S_{\mathrm{Hmax}}\right)$ in this region [Evanzia et al., 2017; Hirschberg et al., 2019; Townend et al., 2012]. However, with the few shear-wave splitting measurements made at only seven OBS stations, it is difficult to reach a firm conclusion regarding the origin of anisotropy. Similar margin-perpendicular fast orientations are reported in the northern Hikurangi margin which are suggested to be a result of closing microcracks due to plate convergence [Zal et al., 2020].
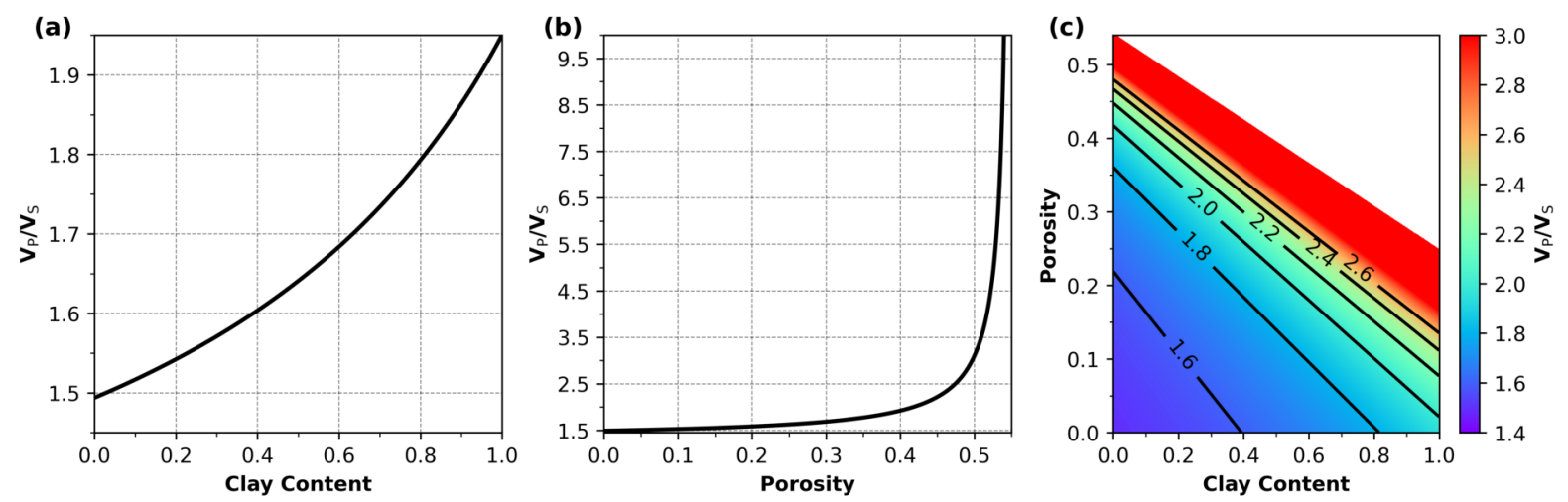

Figure 5.26 $\mathrm{V}_{\mathrm{P}} / \mathrm{V}_{\mathrm{S}}$ variation with porosity and clay content for (a) non-porous, sand and clay mixtures, (b) clean sand (i.e. no clays) and (c) porous, sand and clay mixtures, based on empirical relationships of Castagna et al. [1985]. 
PPS phases converted at the top-sediment basement $\left(\mathrm{PP}_{\mathrm{x}} \mathrm{S}_{\mathrm{b}}\right)$, and the top of the subducting crust $\left(\mathrm{PP}_{\mathrm{x}} \mathrm{S}_{\mathrm{c}}\right)$ indicate coherent delay-times of $\sim 0.07-0.12 \mathrm{sec}$ (Figure $5.27 b)$. Typically, we would expect to see higher delay-times for $\mathrm{PP}_{\mathrm{x}} \mathrm{S}_{\mathrm{c}}$ phases than $\mathrm{PP}_{\mathrm{x}} \mathrm{S}_{\mathrm{b}}$ phases due to the longer travel-path in the former. Except for OBS stations 309 and 315, $\mathrm{PP}_{\mathrm{x}} \mathrm{S}_{\mathrm{b}}$ shows lower delay-times of $\sim 0.07 \mathrm{sec}$ (OBS 313 and 316) than $\mathrm{PP}_{\mathrm{x}} \mathrm{S}_{\mathrm{c}}$ phases (OBS 303, 310 and 328) which have higher delay-times of $\sim 0.08$ to $0.12 \mathrm{sec}$. The similarity of delay-times suggests that the waves have been split in the last segment of their travel-paths (e.g. at the base of the top layer of sediments which is at a depth of $\sim 1-2 \mathrm{~km}$ from the seafloor). Delay-time of $\sim 0.1 \mathrm{sec}$ for a travel-path of $\sim 10-13 \mathrm{~km}$ for $\mathrm{PP}_{\mathrm{x}} \mathrm{S}_{\mathrm{c}}$ (average $\mathrm{V}_{\mathrm{S}}=2.0 \mathrm{~km} / \mathrm{s}$ ) phases represent an anisotropy of only $\sim 1.6-2.0 \%$. However, the likely source of anisotropy here is due to a shorter travel-path of $\sim 2 \mathrm{~km}$. This represents an anisotropy of $\sim 3.5 \%$ (average $\mathrm{V}_{\mathrm{S}}=0.7 \mathrm{~km} / \mathrm{s}$ ) for a delay-time of $\sim 0.1 \mathrm{sec}$ [Silver and Chan, 1988]. A similar localisation of P-wave anisotropy $(>2 \%)$ near active faults within the top $3-4 \mathrm{~km}$ beneath the seafloor is observed in the northern Hikurangi margin [Arai et al., 2020].

Because the anisotropy is mainly confined to the upper $\sim 2 \mathrm{~km}$, it is therefore reasonable to say that the average $V_{\mathrm{P}} / \mathrm{V}_{\mathrm{S}}$ ratio of the sedimentary section above the subducting crust estimated in this study are not affected by anisotropy.

\subsubsection{Average $V_{P} / V_{S}$ Ratio, Lithology, Sedimentary Thickness and Slip-rate Deficit Variations}

Along the Hikurangi margin forearc, three regions with distinct average $V_{\mathrm{P}} / V_{\mathrm{S}}$ ratios for the sedimentary section above the subducting crust can be identified (Figure 5.24c), which suggest the presence of compositionally different material and variations in porosity and/or pore-pressure. These three regions occupy areas of distinct sedimentary thicknesses above the subducting crust (Figure 5.2d) and distinct slip-rate deficits on the subduction thrust (Figure 5.2b, d). Higher average $V_{P} / V_{S}$ ratios are observed where there the sedimentary thickness and slip-rate deficit are low, whereas lower average $\mathrm{V}_{\mathrm{P}} / \mathrm{V}_{\mathrm{S}}$ ratios are observed in the region with the highest sedimentary thickness and slip-rate deficit (Figure 5.24c, d). Average $V_{P} / V_{S}$ ratios show a similar variation to the sedimentary thickness (Figure 5.24c,d). However, the slip-rate deficits do not show rapid changes similar to the $V_{P} / V_{S}$ across the adjacent regions (Figure 5.24c,d). This could be due to the onshore geodetic measurements 


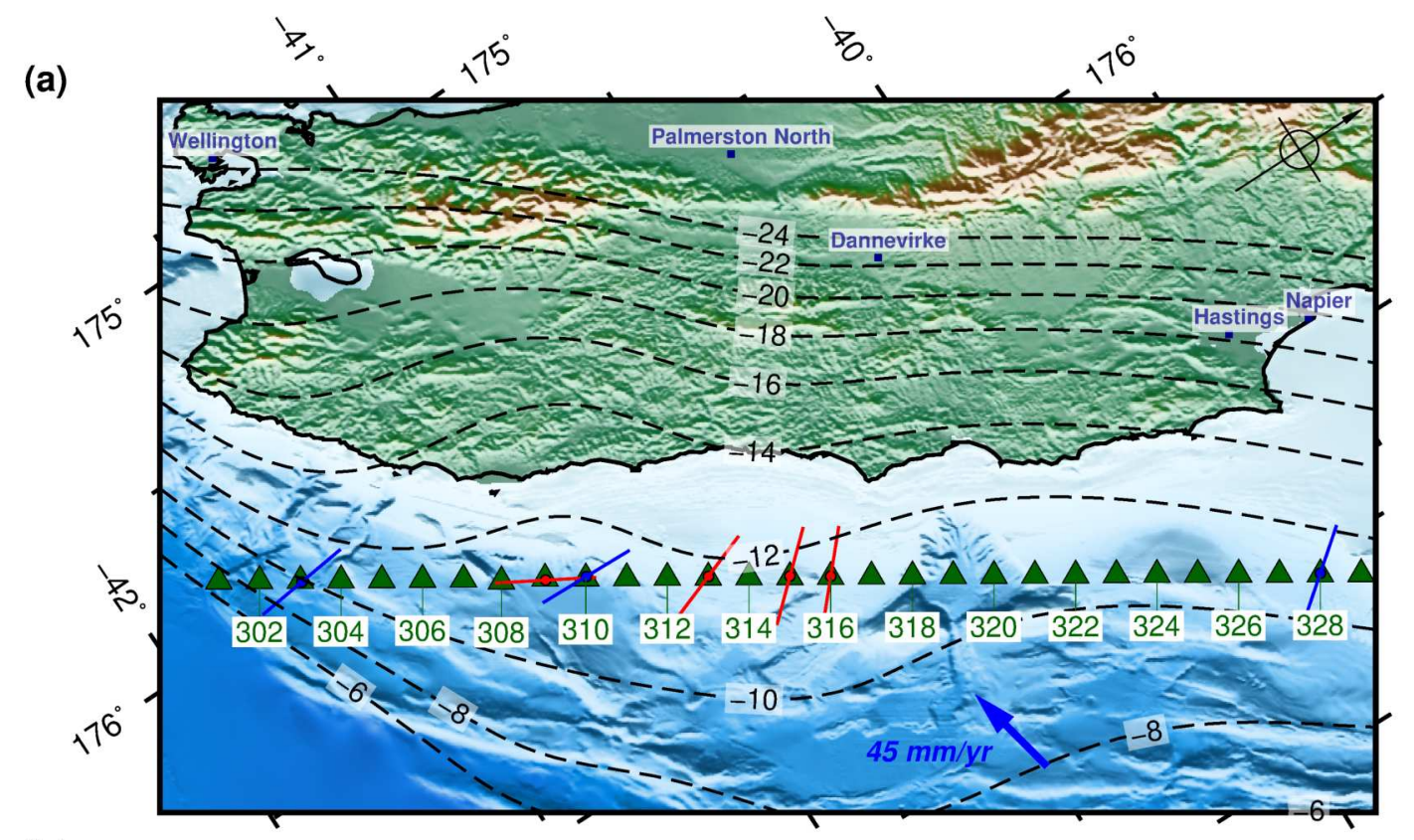

(b)

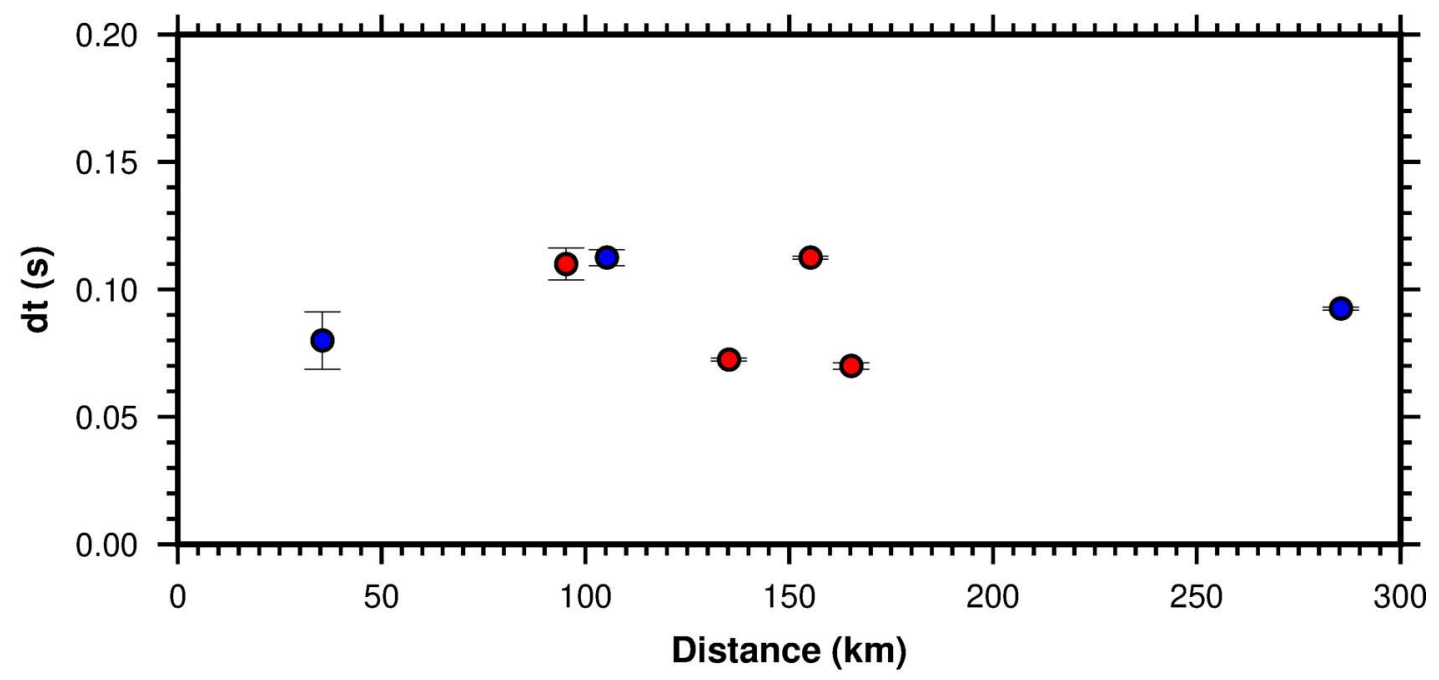

Figure 5.27 (a) Fast shear-wave polarisation azimuths. (b) Delay-times from $\mathrm{PP}_{\mathrm{x}} \mathrm{S}_{\mathrm{b}}$ phases (red) and $\mathrm{PP}_{\mathrm{x}} \mathrm{S}_{\mathrm{c}}$ phases (blue). 
resulting in a lower spatial resolution of the determined slip-rate deficits offshore [Wallace et al., 2012b], or sharp changes in material properties reflected by the average $\mathrm{V}_{\mathrm{P}} / \mathrm{V}_{\mathrm{S}}$ across these regions may result in gradual changes in the slip-rate deficits. This correlation in $V_{\mathrm{P}} / \mathrm{V}_{\mathrm{S}}$ with slip-rate deficit is also observed to match with $\mathrm{V}_{\mathrm{P}} / \mathrm{V}_{\mathrm{S}}$ ratios obtained with adjoint tomographic inversions [Chow et al., 2020].

It is not clear on the extent to which the average $V_{\mathrm{P}} / \mathrm{V}_{\mathrm{S}}$ ratios between the seafloor and the top of the subducting crust can explain the slip-rate deficit on the subduction thrust. Nevertheless, it appears that the combined effects of sediments thickness and lithology (porosity) are reflected in the estimated average $\mathrm{V}_{\mathrm{P}} / \mathrm{V}_{\mathrm{S}}$ ratios. Variations in these parameters may have influenced the effective normal stresses on the subduction thrust. More specifically, a combination of a thicker sedimentary section and low-porosity greywackes of the Pahau Torlesse Terrane may be the contributing factors for the high slip-rate deficits offshore the southeastern North Island (Figure $5.2 \mathrm{~b})$. In contrast, a relatively thinner sedimentary section and the consequent water-saturated porosity and elevated pore-pressures in the sediments of the segments to the southwest and northeast of the strongly coupled plate interface may contribute to low slip-rate deficits. Our interpretation is also consistent with that of Bassett et al. [2014], who suggest that the contrast in P-wave velocities they observe between the southern $\left(\mathrm{V}_{\mathrm{P}}=5.0 \pm 0.2 \mathrm{~km} / \mathrm{s}\right)$ and northern $\left(\mathrm{V}_{\mathrm{P}}=3.5-4.5 \pm 0.1 \mathrm{~km} / \mathrm{s}\right)$ Hikurangi margin forearc sediments reflects higher effective stress levels transmitted through the rock framework. They propose that pore space and conduits are more likely to be localised on faults or sand layers in the southern Hikurangi margin, while in the north, high fluid pressures are maintained by a large fluid inventory from subducting sediment and a pore-space geometry characterised by pervasively fractured rock and mudstone.

\subsection{Conclusions}

From the $\mathrm{V}_{\mathrm{P}} / \mathrm{V}_{\mathrm{S}}$ ratios and shear-wave splitting parameters estimated along the Hikurangi margin forearc we conclude:

1. The top layer of sediments at the ocean bottom along the Hikurangi margin forearc are unconsolidated and water-saturated, indicated by high average $\mathrm{V}_{\mathrm{P}} / \mathrm{V}_{\mathrm{S}}$ between $\sim 2.5 \pm 1.0$ and $\sim 6.0 \pm 2.5$. 
2. Anisotropy $(\sim 3.5 \%)$ is localised in the top layer of sediments beneath the seafloor, with the fast axes oriented perpendicular to the plate interface contours, suggesting stress-aligned fluid-filled cracks control the anisotropy.

3. Average $V_{P} / V_{S}$ ratios of the sedimentary section above the subducting crust vary between $\sim 1.55 \pm 0.08$ and $\sim 2.22 \pm 0.08$.

4. Average $\mathrm{V}_{\mathrm{P}} / \mathrm{V}_{\mathrm{S}}$ ratios of the sedimentary section above the subducting crust show a correlation with the sedimentary thickness, but a weak correlation with slip-rate deficits. Higher $\mathrm{V}_{\mathrm{P}} / \mathrm{V}_{\mathrm{S}}$ ratios are associated with regions of low sedimentary thickness and lower slip-rate deficits where shallow slow slip occurs. Lower $\mathrm{V}_{\mathrm{P}} / \mathrm{V}_{\mathrm{S}}$ ratios are associated with region of higher sedimentary thickness and higher slip-rate deficits. However, the slip-rate deficits do not change rapidly as the $\mathrm{V}_{\mathrm{P}} / \mathrm{V}_{\mathrm{S}}$ ratios.

5. Thicker sediments and rocks above the subduction thrust with higher slip-rate deficits have lower average $\mathrm{V}_{\mathrm{P}} / \mathrm{V}_{\mathrm{S}}$ ratios $(\sim 1.55-1.78)$, indicating the presence of low-porosity sediments, most likely to be Torlesse Terrane greywackes.

6. Relatively thinner, sediments and rocks above the subduction thrust with low slip-rate deficits have higher average $\mathrm{V}_{\mathrm{P}} / \mathrm{V}_{\mathrm{S}}$ ratios $(\sim 1.85-2.22)$, indicating the presence of high-porosity and water-saturated sediments.

\section{Acknowledgements}

SHIRE field team and the crews of $R / V$ Marcus $G$ Langseth and $R / V$ Tangaroa are acknowledged for their time and effort in acquiring the data used in this study. 



\section{Chapter 6}

\section{Thesis Summary}

\subsection{Synthesis}

The Hikurangi subduction margin in New Zealand is characterised by along-strike variations in subduction-thrust slip behaviour and the subduction of an oceanic plateau. A better understanding of the lithospheric structure at the Hikurangi margin is important from a seismic hazard perspective and to improve our understanding of an oceanic plateau, dynamics associated with its subduction and plate tectonics in general.

In this thesis, we explored the offshore lithospheric structure of the Hikurangi margin using controlled-source seismic data acquired during two major experiments, SAHKE and SHIRE. Here, we summarise the findings from the top of the lithosphere, i.e. the top of the overriding Australian Plate down to the base of the subducting Pacific Plate, and their significance in a regional and global context.

Onshore-offshore seismic data from SAHKE, constrains the P-wave velocity structure of the overriding Australian Plate in a swath perpendicular to the southern Hikurangi margin. P-wave velocities and the thickness of the overriding plate vary from the trough to further down-dip of the subduction zone, and therefore provide insights into the composition and structure of the accretionary wedge. Our estimates on the P-wave velocities and structure of the forearc wedge are similar to other studies in the southern Hikurangi margin [e.g. Bassett et al., 2014; Henrys et al., 2013]. However, results from the double-sided onshore-offshore SHIRE transect indicate contrasting P-wave velocities and structure of the forearc wedge in the north. Here, P-wave-speeds are slower and the plate interface dips steeper [Luckie et al., 2020]. This 
is further corroborated by modelling onshore-offshore data along the forearc wedge [Bassett et al., 2014]. We estimated the $\mathrm{V}_{\mathrm{P}} / \mathrm{V}_{\mathrm{S}}$ ratios along the Hikurangi margin forearc using mode-converted phases observed in the ocean bottom seismograph data from SHIRE. The results demonstrate the relationships between $V_{\mathrm{P}} / \mathrm{V}_{\mathrm{S}}$ ratios, crustal composition and slip-rate deficits on the subduction thrust along the Hikurangi margin forearc. A similar $\mathrm{V}_{\mathrm{P}} / \mathrm{V}_{\mathrm{S}}$ variation along the Hikurangi margin is observed from adjoint tomography [Chow et al., 2020]. Our results are analogous to other subduction margins which display variations in plate interface locking and $\mathrm{V}_{\mathrm{P}} / \mathrm{V}_{\mathrm{S}}$ ratios [e.g. Moreno et al., 2014].

Onshore-offshore seismic data from SAHKE further provided constraints on the P-wave velocity structure of the subducting Pacific Plate, which contains the Hikurangi Plateau. Here we constrained the crustal thickness of the Hikurangi Plateau. Our estimates on crustal thickness $(12 \pm 2 \mathrm{~km})$ are consistent with studies in the southern [Henrys et al., 2013; Tozer et al., 2017] and northern Hikurangi margin [Luckie et al., 2020]. On a global context, the Hikurangi Plateau crust is thinner than most other oceanic plateaus. We confirmed the existence of fast upper mantle P-wave-speeds ( $\sim 8.7 \mathrm{~km} / \mathrm{s})$ along margin-perpendicular azimuths, suggesting that the upper mantle has high P-wave-speeds along all azimuths. Similar high upper mantle P-wave-speeds reported for the Ontong-Java and Manihiki Plateaus provide evidence for their association with the Hikurangi Plateau as a single super-plateau [Furumoto et al., 1976; Hochmuth et al., 2019; Stern et al., 2020; Taylor, 2006]. We also detected low P-wave-speed zones in the crust and the upper mantle beneath the Hikurangi trough, which extends down to depths of $\sim 50 \mathrm{~km}$ in the upper mantle. It is interpreted as an example for the subduction-related hydration of an older plate (>100 Myr) with thick crust. We also investigated the origin of a series of wide-angle reflections observed in the same onshore-offshore seismic data, which we interpret as reflections from the lithosphere-asthenosphere boundary zone. Here we find evidence for the existence of an azimuthally anisotropic layer with its fast axis subparallel to the direction of absolute plate motion. It overlies a fluid- or melt-rich, low-velocity channel, which we interpret as the lithosphere-asthenosphere boundary $(\mathrm{LAB})$. The LAB is proposed to be a low-velocity, melt-rich channel by both passiveand controlled-source methods beneath the southern North Island of New Zealand [Stern et al., 2015] and elsewhere [e.g. Fischer et al., 2010; Kawakatsu et al., 2009; 
Mehouachi and Singh, 2018; Rychert et al., 2010]. But the previous studies did not detect the presence of an anisotropic layer above the low-velocity channel. This fine-scale structure of the LAB zone reveal the footprints generated by tectonic plate movement and is key in advancing our knowledge of plate tectonics.

Thus, in summary, the key discoveries on the lithospheric structure at the Hikurangi margin from this thesis are:

1. A $12 \mathrm{~km}$ thick Hikurangi Plateau crust

2. High upper mantle P-wave-speeds $(\sim 8.7 \mathrm{~km} / \mathrm{s})$ of the Hikurangi Plateau

3. Relationships between $\mathrm{V}_{\mathrm{P}} / \mathrm{V}_{\mathrm{S}}$ ratio, composition of the upper plate and slip-rate deficits on the subduction thrust along the Hikurangi margin forearc

4. Hydration of the crust and upper mantle of the Hikurangi Plateau beneath the trough

5. A more structured lithosphere-asthenosphere boundary zone than previously thought

Next, we answer the questions posed in Section 1.2 of the thesis. We also discuss the drawbacks and limitations in each chapter and provide suggestions and directions for future research.

\subsection{Research Questions}

\subsubsection{Chapter 3 - Hydration of the Crust and Upper Mantle of the Hikurangi Plateau as it Subducts at the Southern Hikurangi Margin}

In this chapter, we constrained the crustal and upper mantle structure at the Hikurangi margin along two, single-sided onshore-offshore transects. The research questions posed in this chapter and the answers for those questions revealed from the study are given below.

1. What is the crustal thickness of the Hikurangi Plateau? Is it consistent with estimates from other studies nearby?

From onshore-offshore seismic data, the crustal thickness of the Hikurangi Plateau in the southern Hikurangi margin is estimated to be $\sim 12 \pm 1 \mathrm{~km}$. This is 
consistent with nearby controlled-source seismic experiments [e.g. Henrys et al., 2013; Mochizuki et al., 2019; Tozer et al., 2017].

\section{Is there evidence for anomalously fast upper mantle P-wave-speeds offshore} Hikurangi margin?

Onshore-offshore common receiver gathers show refracted arrivals with apparent P-wave velocities $>9 \mathrm{~km} / \mathrm{s}$ which transitions into an upper mantle layer with a P-wave velocity of $\sim 8.7 \pm 0.2 \mathrm{~km} / \mathrm{s}$ at a depth of $\sim 50 \pm 2 \mathrm{~km}$ in the subducting Pacific Plate. The source-receiver azimuths are margin perpendicular and corroborates previous observations that the upper mantle of the Hikurangi Plateau is characterised by anomalously fast P-wave-speeds along all azimuths.

3. Does the bending-induced normal faulting of the subducting plate near the Hikurangi trough result in the reduction of $P$-wave-speeds in the crust and the upper mantle? What are the implications for crustal and upper mantle hydration?

We find an area of reduced P-wave-speeds in the Hikurangi Plateau crust beneath the trough as it starts to bend under the North Island of New Zealand. The reduction in P-wave-speed is about 10\%. A similar reduction in P-wave-speeds is deduced down to depths of $\sim 50 \mathrm{~km}$ in the upper mantle. The $\sim 10 \%$ reduction in P-wave-speeds in the crust and the upper mantle could be explained in two ways. First, it could be due to the formation of hydrous minerals along the bending-induced normal faults in the crust (e.g. hornblende, chlorite) and upper mantle which can potentially extend down to depths of $\sim 50$ $\mathrm{km}$ in the upper mantle (e.g. antigorite). This is the most widely reported explanation for similar observations at other subduction zones [e.g. Ranero and Sallarès, 2004; Shillington et al., 2015; Van Avendonk et al., 2011]. Alternatively, the $\sim 10 \%$ reduction in $\mathrm{V}_{\mathrm{P}}$ could be due to the development of crack porosity by thermal cracking [Korenaga, 2017]. In contrast to tensional normal faults, if the thermal cracks are open, downward water pumping is possible and this would result in rapid serpentinisation of the thermal cracks. Bending-induced normal faults would further enhance the porosity introduced by thermal cracking. 
This study provided the first insights into the hydration of the crust and upper mantle of the Hikurangi Plateau as it subducts beneath the North Island of New Zealand. It also provided evidence for the presence of anomalously high upper mantle P-wave-speeds offshore of the East Coast of New Zealand. Furthermore, it demonstrated the ability of the onshore-offshore seismic profiling technique in undershooting attenuating areas in the subsurface to obtain constraints on the deeper velocity structure.

The study indicated several limitations as the two onshore-offshore transects were single-sided. Therefore, constraints on near surface velocities onshore could not be accurately estimated. In addition, complementing single-sided onshore-offshore transects with ocean bottom seismographs will be useful in imaging the subsurface at a higher resolution and reduce limitations associated with unreversed shooting.

\subsubsection{Chapter 4- Evidence for a Lithosphere-Asthenosphere Boundary Zone of the Pacific Plate at the Southern Hikurangi Margin}

In this chapter, the series of far-offset, late-arriving wide-angle reflections observed in the onshore-offshore common receiver gathers in Chapter 3 were further investigated. The research questions posed in this chapter and the answers for those questions revealed from the study are given below.

1. Do these wide-angle reflections come from the lithosphere-asthenosphere boundary channel?

Raytracing suggests that the wide-angle reflections define a series of layers with a combined thickness of $11-15 \mathrm{~km}$ at a depth of $\sim 70 \mathrm{~km}$ in the upper mantle. The depth of these layers are consistent with the depth to the lithosphere-asthenosphere boundary channel for a 120 Myr old oceanic plate of 70-80 km [Fischer et al., 2010; Kawakatsu et al., 2009]. These depths are also consistent with the downdip extension of the lithosphere-asthenosphere boundary channel found beneath the southern North Island [Stern et al., 2015].

2. If yes, what do these wide-angle reflections reveal about the lithosphere-asthenosphere boundary?

We explain our observations with the following set of layers: first layer is a $3-5 \mathrm{~km}$ thick, azimuthally anisotropic layer whose fast azimuth subparallels the direction 
of absolute plate motion; second layer is an 8-10 km thick, low P-wave velocity, fluid- or melt-rich layer, which is interpreted to be the lithosphere-asthenosphere boundary channel. Shearing due to the differential motion of the lithosphere on top of the asthenosphere at the lithosphere-asthenosphere boundary channel is proposed to reorient the [100] axes of olivine crystals above it along the direction of maximum finite strain which results in the azimuthally anisotropic layer above; third layer is the asthenosphere.

3. What are the alternative explanations for the wide-angle reflections? Are they plausible?

Our interpretation is non-unique. Tests were therefore conducted to assess whether these observed far-offset, late-arriving phases could be explained by other phenomena. They included (1) peg-leg multiples within the sedimentary column, (2) P- to S- converted phases, and (3) side-swipe. None of these phenomenons could fully explain the characteristics of these far-offset, late-arriving phases.

The study revealed the fine-scale structure at the lithosphere-asthenosphere boundary zone of the Pacific Plate subducting at the Hikurangi margin and is a useful adjunct to controlled-source studies based on near vertical raypaths [Mehouachi and Singh, 2018; Stern et al., 2015]. This has allowed us to discover a more structured LAB zone than previously reported. The most prominent outcome of the study was the evidence for an azimuthally anisotropic layer above a low-velocity, fluid- and/or melt-rich lithosphereasthenosphere boundary. It also provided constraints on the thickness of the Pacific Plate at the Hikurangi margin.

However, the wide-angle reflections were recorded only at two stations with a good signal-to-noise ratio, which limited the tracking of the lithosphere-asthenosphere boundary zone further across the margin. In addition, the assumption of a a two-dimensional geometry of the subduction zone to project three-dimensional source-receiver raypaths on to a two-dimensional model may have had some effect on the amplitudes of the synthetic common receiver gathers. But it is unavoidable as the generation of synthetic seismograms for a three-dimensional Earth model at controlled-source seismic frequencies $(>10 \mathrm{~Hz})$ is extremely computationally 
expensive, when compared to a two-dimensional model.

Similar wide-angle reflections from the lithospheric mantle are observed in the onshore-offshore data from the Nankai margin [D. Bassett, pers. comm., 31 July 2020]. Although there is a high density of permanent seismograph stations in Japan, the reflections are observed only at the highest gain stations. Common receiver gathers at adjacent stations (20 km apart) from the airgun shots of the same multichannel seismic line show vastly different resolution of mantle reflections.

Our study has motivated colleagues to look at similar wide-angle reflections in onshore-offshore data at other regions (e.g. at Nankai margin in Japan), which will be influential in improving our understanding of the lithosphere-asthenosphere boundary. It would also be useful in designing future controlled-source experiments targeting the lithosphere-asthenosphere boundary.

\subsubsection{Chapter $5-\mathrm{V}_{\mathrm{P}} / \mathrm{V}_{\mathrm{S}}$ Ratio, Material and Slip-rate Deficit Variations along Hikurangi Margin Forearc}

In this chapter, mode-converted waves from airgun shots recorded by an array of ocean bottom seismographs along the Hikurangi margin forearc were used to estimate the average $V_{P} / V_{S}$ ratio of the sediments and rocks above the subducting crust. Here we answer the following questions raised at the beginning of the thesis.

1. How does the $V_{P} / V_{S}$ ratio of the sediments and rocks above the subducting crust vary along the Hikurangi margin forearc? Does it show any relationship with the crustal composition and the subduction thrust slip behaviour?

Average $\mathrm{V}_{\mathrm{P}} / \mathrm{V}_{\mathrm{S}}$ ratios of the sediments and rocks above the subducting crust vary between $\sim 1.55 \pm 0.08$ and $\sim 2.22 \pm 0.08$. They show an along-strike variation with three regions of distinct $\mathrm{V}_{\mathrm{P}} / \mathrm{V}_{\mathrm{S}}$ ratios. In the southernmost section of the ocean bottom seismograph array (between $0 \mathrm{~km}$ and $\sim 80 \mathrm{~km}$ model distances), the average $V_{\mathrm{P}} / \mathrm{V}_{\mathrm{S}}$ ratios are high (mean average $\mathrm{V}_{\mathrm{P}} / \mathrm{V}_{\mathrm{S}}=2.00$ ). In between $\sim 80$ $\mathrm{km}$ and $\sim 160 \mathrm{~km}$ model distances the mean average $\mathrm{V}_{\mathrm{P}} / \mathrm{V}_{\mathrm{S}}$ ratio reduces down to 1.67. The mean average $V_{P} / V_{S}$ ratio increases again to 2.08 between $\sim 160$ and $\sim 300 \mathrm{~km}$. These values represent variable sediment (and rock) thickness, crustal composition along the strike, and also effects of porosity, clay content and pore-pressure. In particular the region with low mean average $V_{P} / V_{S}$ ratio 
corresponds to a region found to be comprising of low-porosity greywackes. We also observe a notable correlation between sediment thickness and the average $\mathrm{V}_{\mathrm{P}} / \mathrm{V}_{\mathrm{S}}$ ratios of the rocks and sediments above the subducting crust and the slip-rate deficits. There is also a weak correlation between the average $V_{P} / V_{S}$ ratios of the rocks and sediments above the subducting crust and the slip-rate deficits. In regions with higher sediment (and rock) thicknesses and high slip-rate deficits, the average $\mathrm{V}_{\mathrm{P}} / \mathrm{V}_{\mathrm{S}}$ ratios are low and in regions with relatively lower sediment (and rock) thicknesses and low slip-rate deficits, the average $\mathrm{V}_{\mathrm{P}} / \mathrm{V}_{\mathrm{S}}$ ratios are high.

2. How does the anisotropy of the sediments and rocks above the subducting crust vary along the forearc? Are the $V_{P} / V_{S}$ ratios affected by anisotropy?

Shear-wave splitting results indicate delay-times of 0.07-0.12 sec and shear-wave polarisation azimuths perpendicular to the plate interface contours. This suggests that an anisotropy $(\sim 3.5 \%)$ is localised in the top layer of sediments beneath the seafloor. The fast shear-wave polarisation azimuths oriented perpendicular to the plate interface contours suggest that stress-aligned fluid-filled cracks control the anisotropy.

Average $V_{\mathrm{P}} / \mathrm{V}_{\mathrm{S}}$ ratios estimated from this study can be used to calculate the shear-wave velocities $\left(V_{S}\right)$ combining with the compressional-wave $\left(V_{P}\right)$ model derived independently from the tomographic inversion of first arrivals. An understanding of $\mathrm{V}_{\mathrm{S}}$ is particularly important for performing dynamic rupture simulations of earthquakes and predicting associated ground shaking in assessing seismic risk. $V_{P}$ and $V_{S}$ are directly related to the bulk modulus $(\kappa)$ and shear modulus $(\mu)$, and can be used to estimate the strength of the upper plate and provide insights into its competency with plate convergence.

However, average $V_{P} / V_{S}$ ratios have been estimated only beneath ocean bottom seismographs where mode-converted phases could be observed. Therefore, the spatial resolution of $V_{P} / V_{S}$ ratios is limited. The average $V_{P} / V_{S}$ ratios estimated are for the section of sediments and rocks between the OBS station and the conversion interface (e.g top of the subducting crust). Thus they would not reflect any $V_{\mathrm{P}} / \mathrm{V}_{\mathrm{S}}$ variations due to fine-scale layering between the ocean bottom seismograph and the 
converting interface (lower vertical resolution).

In this research project, several likely PSS and/or PSP mode-conversions could be identified. But they were not used in the study as they could not be confirmed as such mode-conversions. Further investigations into these potential mode-conversions could be conducted. If confirmed they can be used to further fine-tune the $V_{P} / V_{S}$ ratios using conventional raytrace methods. This will also be useful in increasing the spatial and vertical resolutions of $\mathrm{V}_{\mathrm{P}} / \mathrm{V}_{\mathrm{S}}$ ratios.

\subsection{Concluding Remarks}

The findings presented in this thesis provide new insights into the material and processes from the top to the bottom of an oceanic plate. The thesis sheds light into the lithospheric composition, geodynamic processes and the seismic signatures associated with the subduction of an oceanic plateau. A wider question is, "Are the phenomena we see here specifically related only to the subduction of a oceanic plateau with thick crust, or are these processes common to all subduction zones?". From similar studies from other parts of the world [e.g. Mehouachi and Singh, 2018; Moreno et al., 2014; Shillington et al., 2015], it appears that what we see at the Hikurangi margin has parallels elsewhere and the research presented here contributes to our understanding of plate tectonics in general. 

Appendices 

Appendix A

Supplementary Material for Chapter 3 


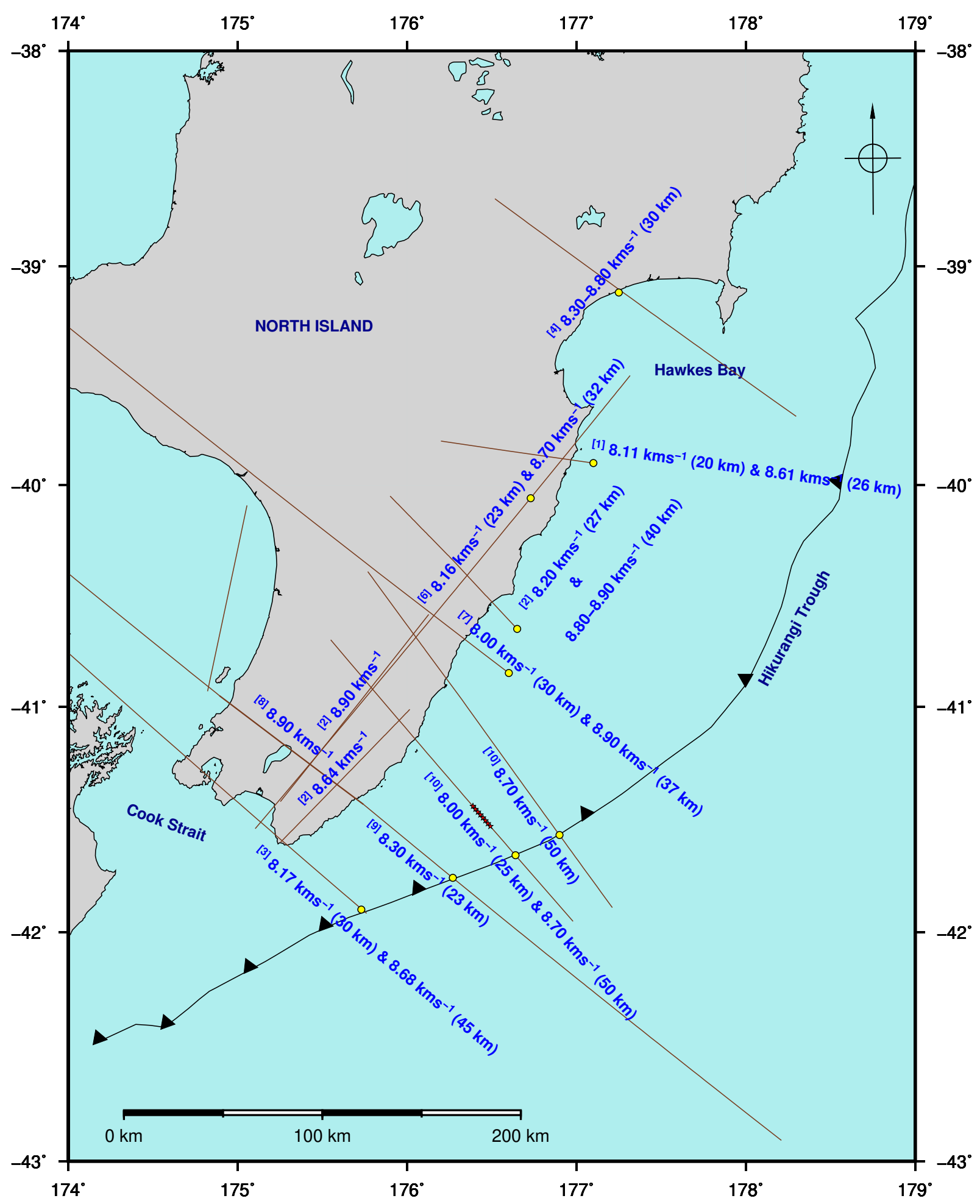

Figure A.1 Upper mantle P-wave-speeds and their depths reported by previous authors and in this study along PEGASUS23 and PEGASUS25 transects. Brown lines indicate the profiles of the velocity models by the authors. Yellow circles-Locations of P-wave-speeds of the upper manlte. P-wave-speeds are indicated in blue with their depths inside parantheses. The orientation of the P-wave-speed labels indicate their azimuth of measurement. Black line-Hikurangi trough. Black triangles indicate thrust direction. [1] Chong [1982], [2] Kayal and Smith [1984], [3] Robinson [1986], [4] Bannister [1988], [5] Galea [1992], [6] Chadwick [1997], [7] Eberhart-Phillips et al. [2010], [8] Stern et al. [2018], [9] Mochizuki et al. [2019], [10] This study. 


\section{A.1 Pre-modelling Considerations}

\section{A.1.1 Defining the Transects}

Due to difficulties in reaching specific locations in the field (e.g. terrain, waterways, accessibility) the onshore seismographs could not be placed inline with the offshore MCS lines (Figure 3.1b). Therefore, to model the wide-angle reflections and refractions observed in the receiver gathers in terms of a 2D model, the onshore seismographs are projected perpendicularly to the transect defined by a line that is a linear regression of the individual airgun shots of a seismic line. In order to preserve the true sourcereceiver offsets, the airgun shots are radially projected on to the regression line (Figure A.2).

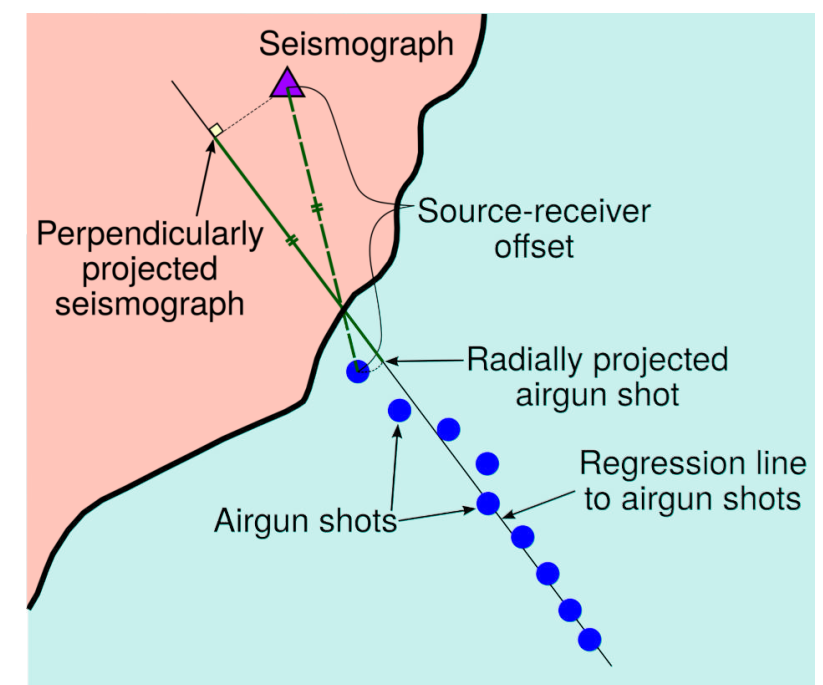

Figure A.2 Perpendicular projection of seismographs and radial projection of airgun shots to the transect defined by the linear regression line to the airgun shots. This projection preserves true source-receiver offsets.

\section{A.1.2 Projection of SAHKE 01 airgun shots to PEGASUS23 and PEGASUS25 tran- sects}

SAHKE01 line deviates $10^{\circ}$ from the PEGASUS23, and $15^{\circ}$ from PEGASUS25 transects. An implicit assumption in interpreting the gathers produced from SAHKE01 airgun shots, in terms of 2D models along PEGASUS23 and 25 transects, is that the crustal structure beneath the region defined by the surface projection of the ray-paths does not vary. This $3 \mathrm{D}$ to $2 \mathrm{D}$ projection issue is further minimised by only using the shots that have source-receiver offsets greater than $150 \mathrm{~km}$ for MRZ, $100 \mathrm{~km}$ for PEG3 and $130 \mathrm{~km}$ for BFZ (Figure 3.1b). 
When the subsurface interfaces from which refractions occur are horizontal, the velocity of the refracting layer is resolved from the gradient of a $t-x$ plot. The velocity determination here is a function of source-receiver offset only. Therefore, a change in source-receiver azimuth from shot line would not affect velocity determinations as long as true source-receiver offsets are preserved.

When the interfaces are dipping, the velocity determination is a function of both offset and dip of the interface. A deviation in the source-receiver azimuth from the true structural dip of an interface would result in sampling the apparent dip of the interface (Equation A.1 [Dobrin, 1988]).

$$
\sin \phi=\cos \psi \cdot \sin \delta
$$

Here, $\phi$ is the apparent dip, $\psi$ is the angle between the shot direction and the direction of the true dip, $\delta$ is the true dip.

The azimuths of PEGASUS23 and PEGASUS25 transects can be considered to be inline with the true dip of the plate interface. The dip angle is $\sim 3-5^{\circ}$ [Williams et al., 2013]. The interfaces further beneath are more likely to be parallel to the plate interface and their true dips can also be considered to be inline with the two transects.

The source and receiver pair with the maximum deviation in azimuth from the true dip are the airgun shot at $130 \mathrm{~km}$ offset from BFZ station and the BFZ station itself (Figure $3.1 b)$, respectively. This deviation in azimuth is $\sim 28^{\circ}$.

Consider a two-layer model, with an upper velocity of $8.0 \mathrm{~km} / \mathrm{s}$ and a lower velocity of $8.7 \mathrm{~km} / \mathrm{s}$ separated by a dipping interface. The difference between the apparent velocities of the refracted phase for different angles of true dip when source-receiver azimuth is along the true-dip and when the source-receiver azimuth deviates from the true dip azimuth, in a shooting up-dip case is shown in Figure A.3. For an interface with a true dip of $5^{\circ}$, which is sampled by a set of source, receiver pairs whose azimuth deviates $28^{\circ}$ from the true dip azimuth, the difference in apparent velocity is $\sim 0.05 \mathrm{~km} / \mathrm{s}$ (Figure A.3). This difference is very small and can be considered negligible. In the case of this study, the deviation of the azimuth of the source-receiver pair from the azimuth of the transect (true-dip azimuth) decreases as 
the source-receiver offset increases. Thus the difference in apparent velocity further decreases.

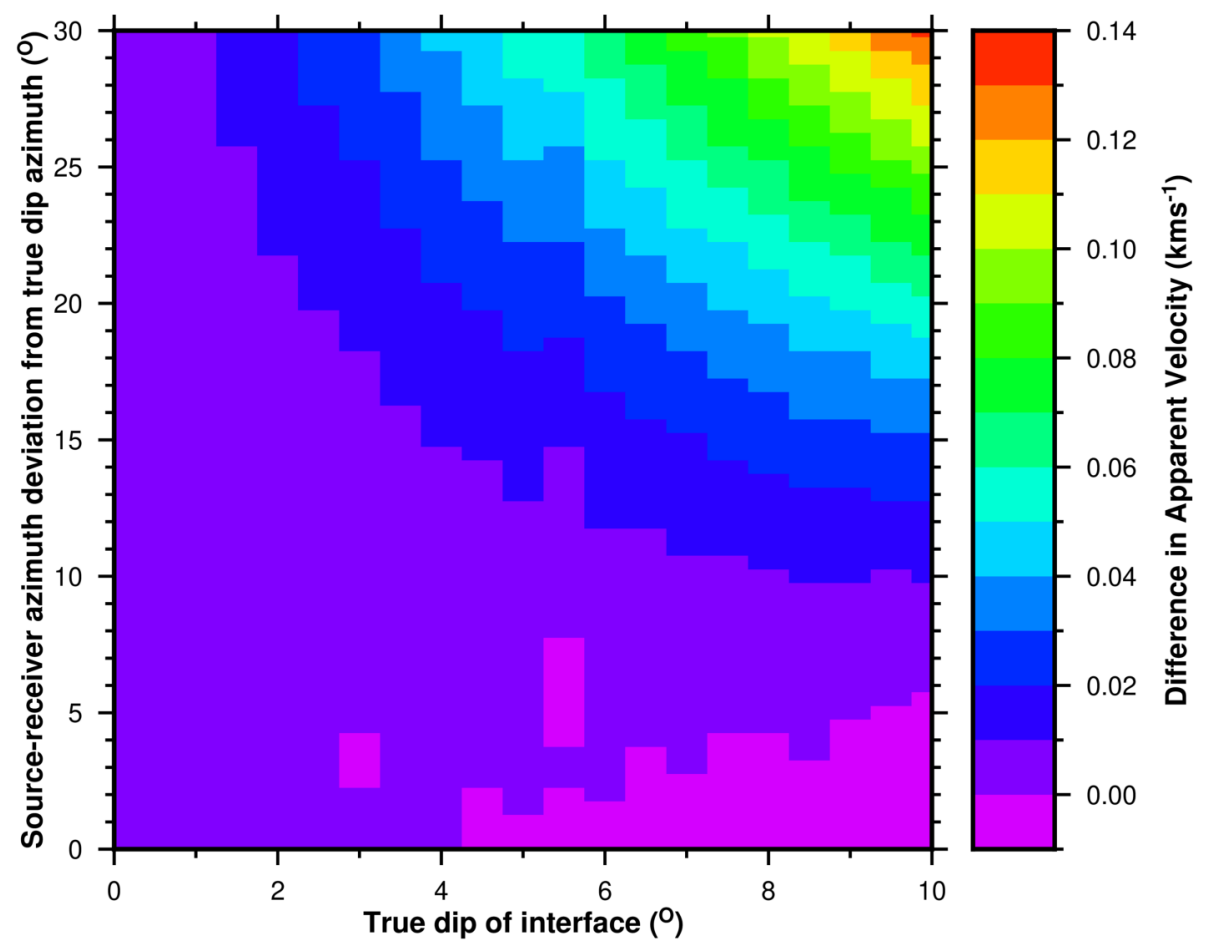

Figure A.3 Difference between the apparent velocities of a refracted phase for different true-dip angles when the source-receiver azimuth is along the true-dip and when the source-receiver azimuth deviates from the true dip azimuth. A two layer case with upper velocity of $8.0 \mathrm{~km} / \mathrm{s}$ and lower velocity of 8.7 $\mathrm{km} / \mathrm{s}$ when shooting up-dip is considered here. 
Table A.1 Original and projected location details of seismographs along PEGASUS23 and PEGASUS25 transects. Deviation gives the difference in the horizontal location and in the elevation between the original and projected locations. All X and Y coordinates are in New Zealand Transverse Mercator 2000 projected coordinate system. $\mathrm{Z}$ is the elevation. Loc. = location. Model $\mathrm{X}$ is the $\mathrm{x}$-distance of the seismograph along the two-dimensional velocity models.

\begin{tabular}{|c|c|c|c|c|c|c|c|c|c|c|}
\hline \multirow{2}{*}{ Transect } & \multirow{2}{*}{ Station } & \multicolumn{3}{|c|}{ Original location } & \multicolumn{3}{|c|}{ Projected location } & \multicolumn{2}{|c|}{ Deviation } & \multirow{2}{*}{ Model X (km) } \\
\hline & & $X(\mathrm{~m})$ & $\mathrm{Y}(\mathrm{m})$ & $\mathrm{Z}(\mathrm{m})$ & $X(\mathrm{~m})$ & $\mathrm{Y}(\mathrm{m})$ & $\mathrm{Z}(\mathrm{m})$ & Loc. $(\mathrm{km})$ & $\mathrm{Z}(\mathrm{m})$ & \\
\hline \multirow{12}{*}{ 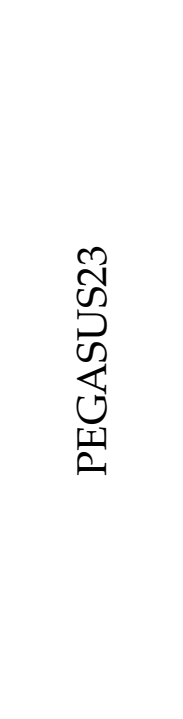 } & MRZ & 1817978.05 & 5495727.41 & 292.79 & 1814316.63 & 5492806.82 & 611.64 & 4.683 & -318.85 & 0.000 \\
\hline & HOWZ & 1811860.23 & 5469594.71 & 342.95 & 1824677.12 & 5479818.32 & 240.42 & 16.395 & 102.53 & 16.614 \\
\hline & PEG13 & 1828796.73 & 5473041.19 & 247.75 & 1829582.84 & 5473668.24 & 309.76 & 1.006 & -62.01 & 24.481 \\
\hline & TIWZ & 1843523.34 & 5482096.23 & 434.55 & 1830895.11 & 5472023.11 & 315.05 & 16.154 & 119.50 & 26.586 \\
\hline & PEG12 & 1834317.90 & 5466320.67 & 146.66 & 1835005.96 & 5466869.52 & 167.06 & 0.880 & -20.40 & 33.178 \\
\hline & PEG11 & 1841084.49 & 5461724.03 & 129.39 & 1839877.00 & 5460761.65 & 283.26 & 1.543 & -153.87 & 40.991 \\
\hline & PEG5 & 1843716.77 & 5454823.62 & 223.54 & 1844265.46 & 5455261.29 & 256.59 & 0.702 & -33.05 & 48.027 \\
\hline & PEG4 & 1845264.89 & 5452508.90 & 245.85 & 1845995.85 & 5453091.96 & 255.59 & 0.935 & -9.74 & 50.802 \\
\hline & PEG3 & 1847899.25 & 5449729.12 & 331.55 & 1848375.35 & 5450108.88 & 351.86 & 0.609 & -20.31 & 54.618 \\
\hline & TMWZ & 1842714.94 & 5445382.66 & 368.43 & 1848478.26 & 5449979.87 & 346.49 & 7.372 & 21.94 & 54.783 \\
\hline & PEG2 & 1853744.92 & 5444808.79 & 69.16 & 1853047.10 & 5444252.12 & 76.29 & 0.893 & -7.13 & 62.110 \\
\hline & PEG1 & 1854993.59 & 5441510.29 & 19.65 & 1855140.62 & 5441627.57 & 20.00 & 0.188 & -0.35 & 65.476 \\
\hline \multirow{9}{*}{ 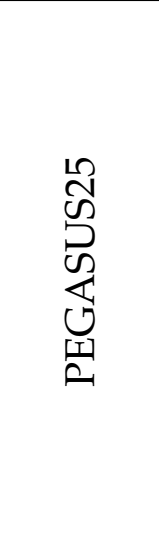 } & PRWZ & 1851558.35 & 5506430.52 & 261.61 & 1849637.23 & 5505163.92 & 408.93 & 2.301 & -147.32 & 0.000 \\
\hline & PEG10* & 1856441.82 & 5493665.93 & 238.03 & 1856987.35 & 5494015.60 & 239.26 & 0.653 & -1.23 & 13.353 \\
\hline & PEG9* & 1861839.16 & 5487890.32 & 330.90 & 1861272.20 & 5487516.53 & 340.06 & 0.679 & -9.16 & 21.138 \\
\hline & PEG8 & 1861285.42 & 5484829.44 & 196.24 & 1862511.05 & 5485637.50 & 259.99 & 1.468 & -63.75 & 23.388 \\
\hline & BFZ & 1874354.07 & 5491735.13 & 285.87 & 1863297.11 & 5484445.25 & 254.01 & 13.244 & 31.86 & 24.752 \\
\hline & PEG7 & 1864937.83 & 5479539.03 & 166.93 & 1866048.85 & 5480271.53 & 151.53 & 1.331 & 15.40 & 29.816 \\
\hline & PEG6.5* & 1866967.40 & 5476827.81 & 83.29 & 1867909.71 & 5477449.07 & 236.65 & 1.129 & -153.36 & 33.196 \\
\hline & PEG6 & 1870075.63 & 5472278.70 & 58.12 & 1870941.97 & 5472849.88 & 59.52 & 1.038 & -1.40 & 38.705 \\
\hline & CPWZ & 1869646.42 & 5466319.54 & 108.92 & 1873550.44 & 5468893.47 & -13.07 & 4.676 & 121.99 & 43.444 \\
\hline
\end{tabular}

* Signal-to-noise ratio is very low at these stations and do not show any distinguishable arrivals. 
(a)
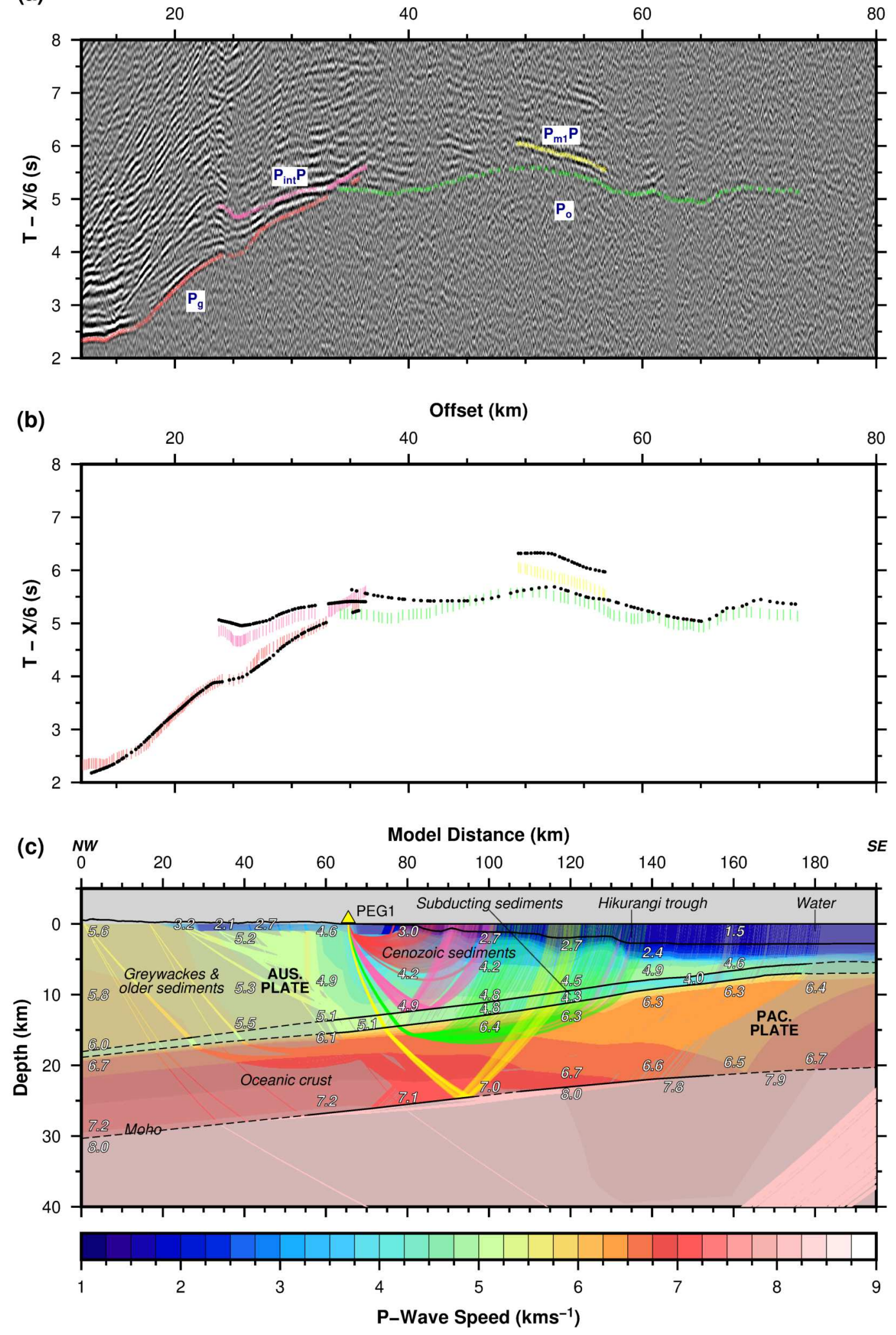

Figure A.4 (a) Common receiver gather at PEG1 station with airgun shots of PEGASUS23 line. Different seismic phases are colour-coded. $\mathrm{P}_{\mathrm{g}}$-red, $\mathrm{P}_{\text {int }} \mathrm{P}$-pink, $\mathrm{P}_{\mathrm{o}}$-light green, $\mathrm{P}_{\mathrm{m} 1} \mathrm{P}$-yellow and $\mathrm{P}_{\mathrm{n} 1}$-cyan. (b) Observed travel-times (colour-coded as in (a)) and calculated travel-times (black dots). The height of an observed travel-time pick is twice its pick uncertainty. Note that the travel-time axes of (a) and (b) are reduced at $6 \mathrm{~km} / \mathrm{s}$. (c) Traced rays and the velocity model, with rays colour-coded as in (a) and (b). Yellow triangle-location of seismograph. Grey shading indicates areas in the velocity model without ray coverage. White numbers with black outline are P-wave-speeds at those locations in $\mathrm{km} / \mathrm{s}$. Dashed black lines are inferred interfaces. Continuous black lines are confirmed interfaces. The x-axes in (a) and (b) indicate the offset from the recording station and the x-axis in (c) indicates the model distance starting from MRZ station. AUS. PLATE and PAC. PLATE are Australian Plate and Pacific Plate respectively. 

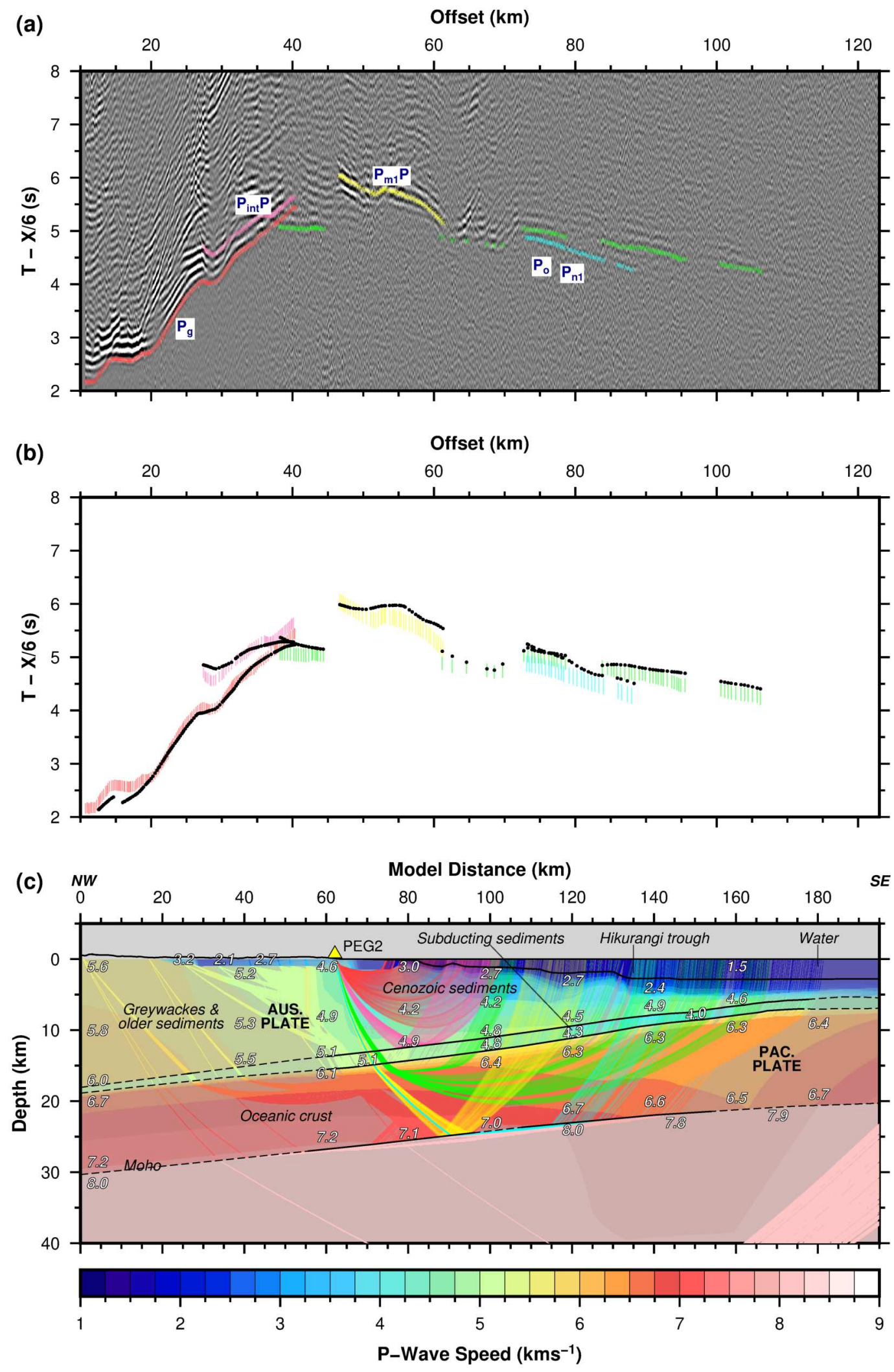

Figure A.5 (a) Common receiver gather at PEG2 station with airgun shots of PEGASUS23 line. Different seismic phases are colour-coded. $\mathrm{P}_{\mathrm{g}}$-red, $\mathrm{P}_{\text {int }} \mathrm{P}$-pink, $\mathrm{P}_{\mathrm{o}}$-light green, $\mathrm{P}_{\mathrm{m} 1} \mathrm{P}$-yellow and $\mathrm{P}_{\mathrm{n} 1}$-cyan. (b) Observed travel-times (colour-coded as in (a)) and calculated travel-times (black dots). The height of an observed travel-time pick is twice its pick uncertainty. Note that the travel-time axes of (a) and (b) are reduced at $6 \mathrm{~km} / \mathrm{s}$. (c) Traced rays and the velocity model, with rays colour-coded as in (a) and (b). Yellow triangle-location of seismograph. Grey shading indicates areas in the velocity model without ray coverage. White numbers with black outline are P-wave-speeds at those locations in $\mathrm{km} / \mathrm{s}$. Dashed black lines are inferred interfaces. Continuous black lines are confirmed interfaces. The x-axes in (a) and (b) indicate the offset from the recording station and the $\mathrm{x}$-axis in (c) indicates the model distance starting from MRZ station. AUS. PLATE and PAC. PLATE are Australian Plate and Pacific Plate respectively. 

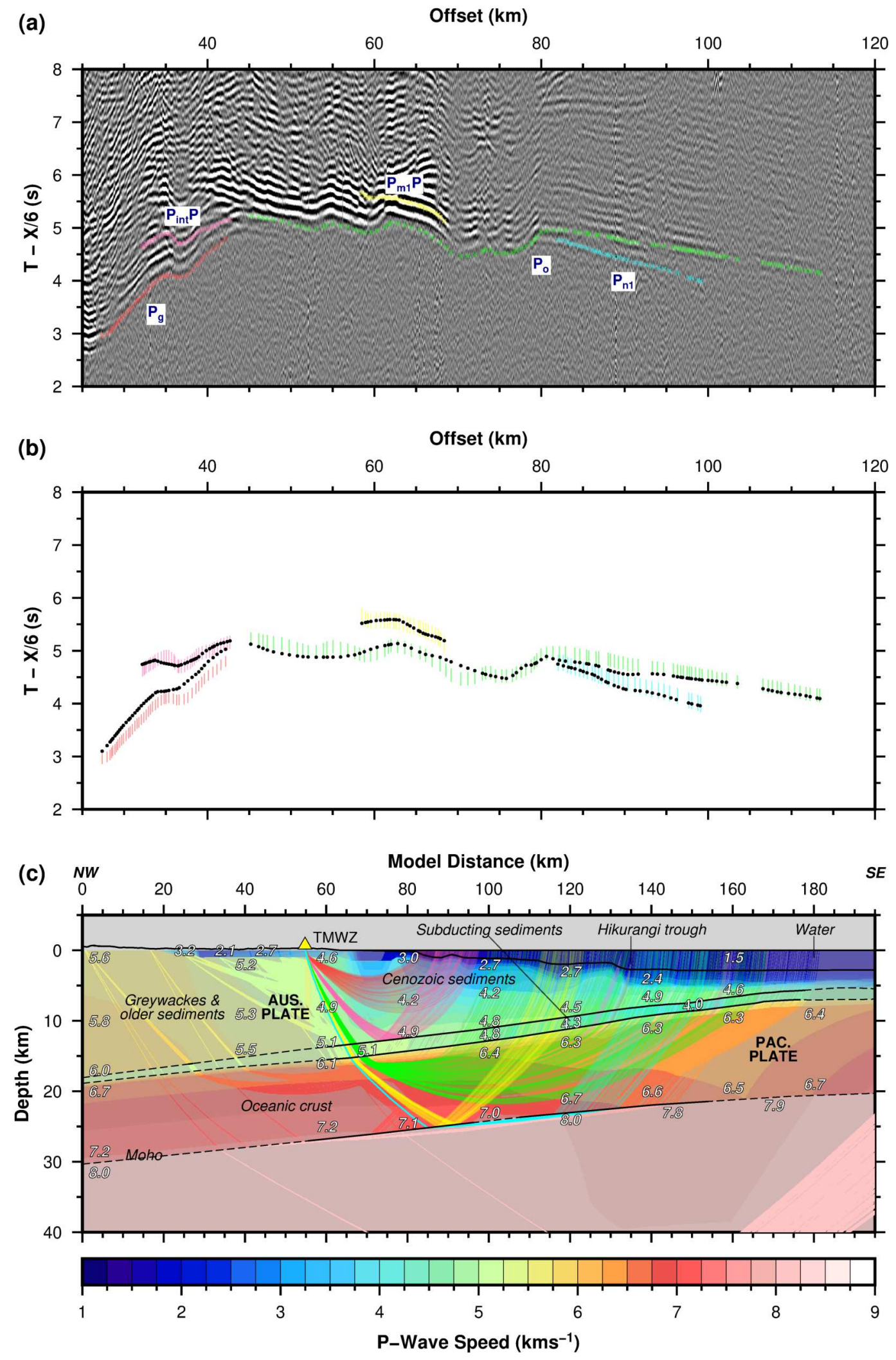

Figure A.6 (a) Common receiver gather at TMWZ station with airgun shots of PEGASUS23 line. Different seismic phases are colour-coded. $\mathrm{P}_{\mathrm{g}}$-red, $\mathrm{P}_{\text {int }} \mathrm{P}$-pink, $\mathrm{P}_{\mathrm{o}}$-light green, $\mathrm{P}_{\mathrm{m} 1} \mathrm{P}$-yellow and $\mathrm{P}_{\mathrm{n} 1}$-cyan. (b) Observed travel-times (colour-coded as in (a)) and calculated travel-times (black dots). The height of an observed travel-time pick is twice its pick uncertainty. Note that the travel-time axes of (a) and (b) are reduced at $6 \mathrm{~km} / \mathrm{s}$. (c) Traced rays and the velocity model, with rays colour-coded as in (a) and (b). Yellow triangle-location of seismograph. Grey shading indicates areas in the velocity model without ray coverage. White numbers with black outline are P-wave-speeds at those locations in $\mathrm{km} / \mathrm{s}$. Dashed black lines are inferred interfaces. Continuous black lines are confirmed interfaces. The x-axes in (a) and (b) indicate the offset from the recording station and the $x$-axis in (c) indicates the model distance starting from MRZ station. AUS. PLATE and PAC. PLATE are Australian Plate and Pacific Plate respectively. 
(a)

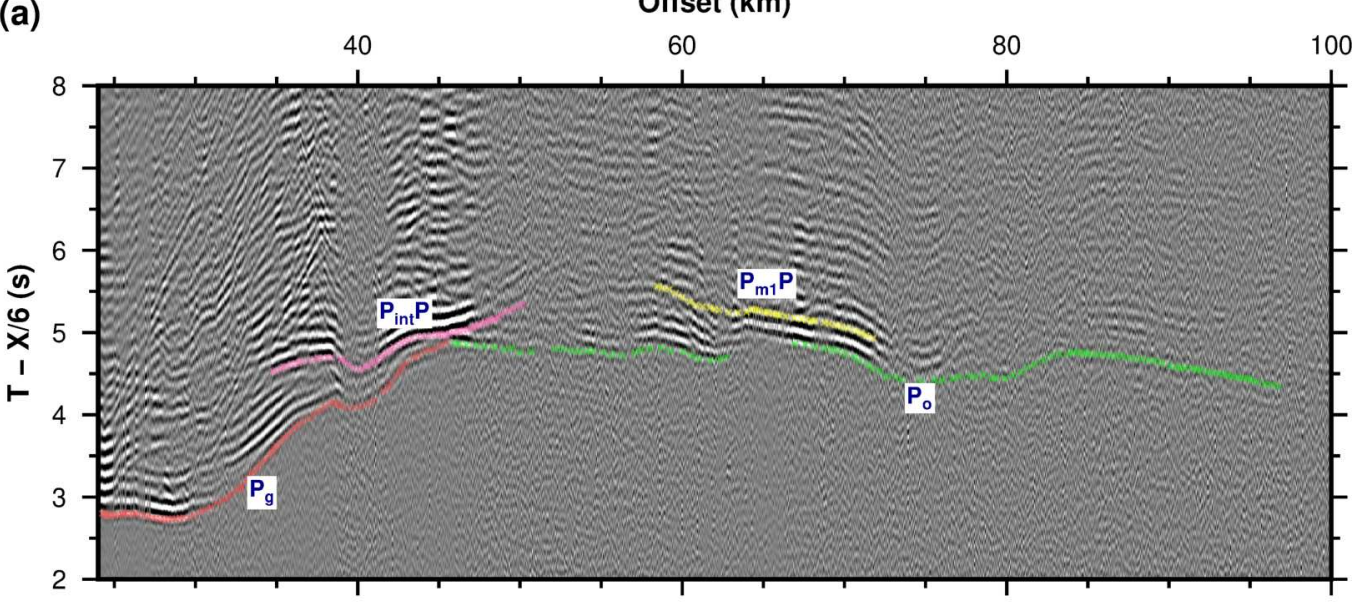

(b)
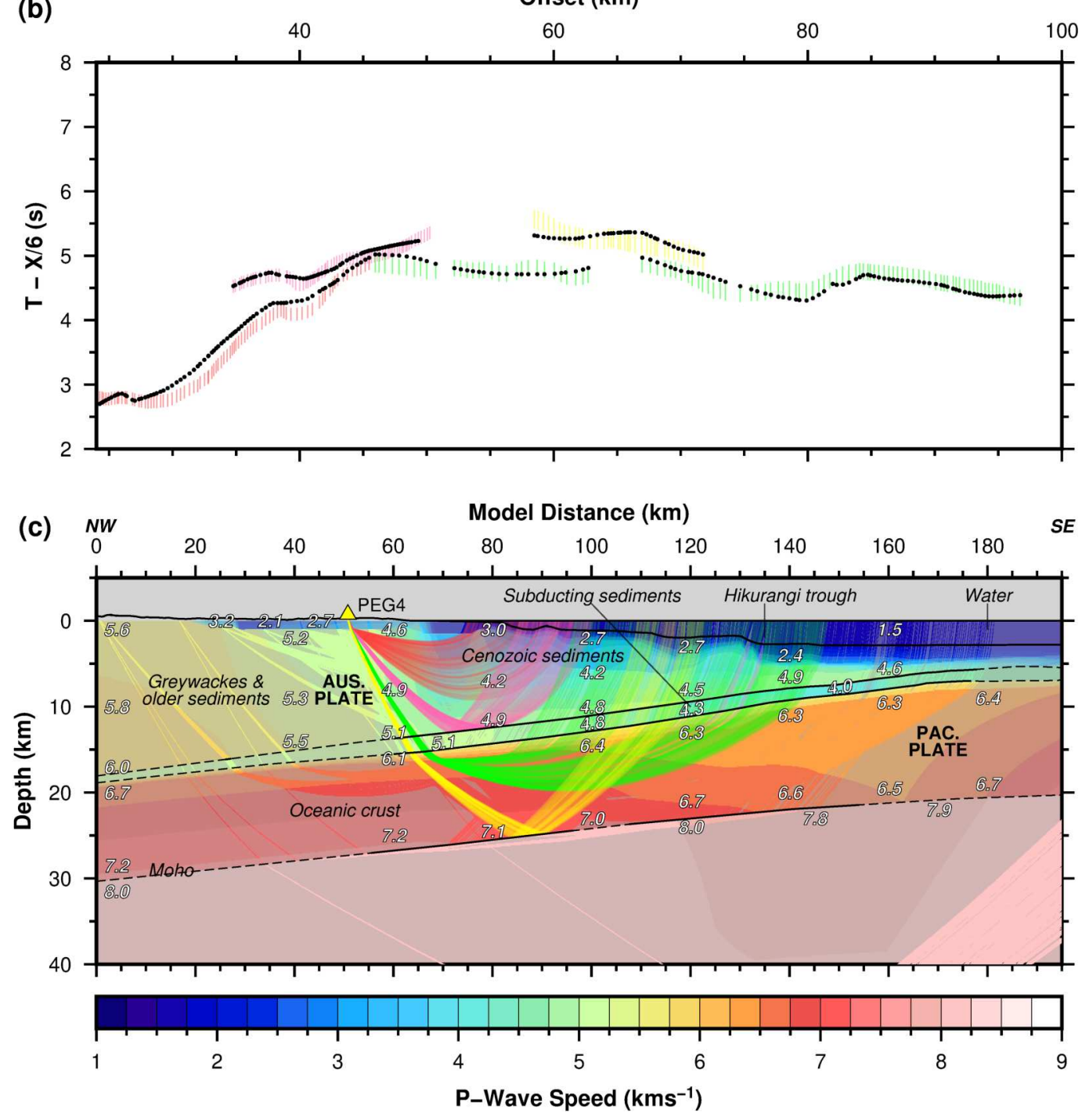

Figure A.7 (a) Common receiver gather at PEG4 station with airgun shots of PEGASUS23 line. Different seismic phases are colour-coded. $\mathrm{P}_{\mathrm{g}}$-red, $\mathrm{P}_{\text {int }} \mathrm{P}$-pink, $\mathrm{P}_{\mathrm{o}}$-light green, $\mathrm{P}_{\mathrm{m} 1} \mathrm{P}$-yellow and $\mathrm{P}_{\mathrm{n} 1}$-cyan. $(\mathbf{b})$ Observed travel-times (colour-coded as in (a)) and calculated travel-times (black dots). The height of an observed travel-time pick is twice its pick uncertainty. Note that the travel-time axes of (a) and (b) are reduced at $6 \mathrm{~km} / \mathrm{s}$. (c) Traced rays and the velocity model, with rays colour-coded as in (a) and (b). Yellow triangle-location of seismograph. Grey shading indicates areas in the velocity model without ray coverage. White numbers with black outline are P-wave-speeds at those locations in $\mathrm{km} / \mathrm{s}$. Dashed black lines are inferred interfaces. Continuous black lines are confirmed interfaces. The x-axes in (a) and (b) indicate the offset from the recording station and the $x$-axis in (c) indicates the model distance starting from MRZ station. AUS. PLATE and PAC. PLATE are Australian Plate and Pacific Plate respectively. 

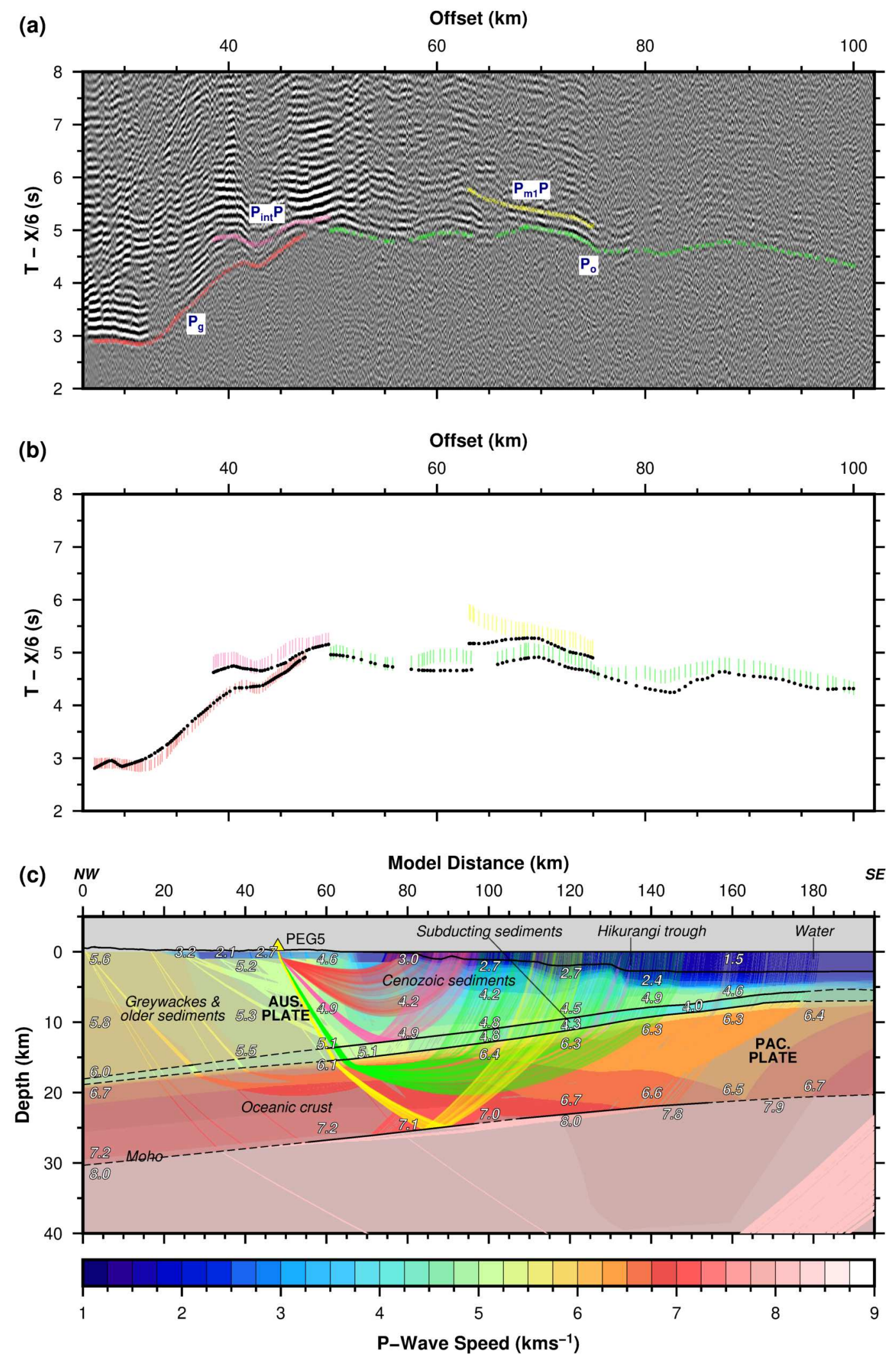

Figure A.8 (a) Common receiver gather at PEG5 station with airgun shots of PEGASUS23 line. Different seismic phases are colour-coded. $\mathrm{P}_{\mathrm{g}}$-red, $\mathrm{P}_{\text {int }} \mathrm{P}$-pink, $\mathrm{P}_{\mathrm{o}}$-light green, $\mathrm{P}_{\mathrm{m} 1} \mathrm{P}$-yellow and $\mathrm{P}_{\mathrm{n} 1}$-cyan. - (b) Observed travel-times (colour-coded as in (a)) and calculated travel-times (black dots). The height of an observed travel-time pick is twice its pick uncertainty. Note that the travel-time axes of (a) and (b) are reduced at $6 \mathrm{~km} / \mathrm{s}$. (c) Traced rays and the velocity model, with rays colour-coded as in (a) and (b). Yellow triangle-location of seismograph. Grey shading indicates areas in the velocity model without ray coverage. White numbers with black outline are P-wave-speeds at those locations in $\mathrm{km} / \mathrm{s}$. Dashed black lines are inferred interfaces. Continuous black lines are confirmed interfaces. The x-axes in (a) and (b) indicate the offset from the recording station and the $x$-axis in (c) indicates the model distance starting from MRZ station. AUS. PLATE and PAC. PLATE are Australian Plate and Pacific Plate respectively. 

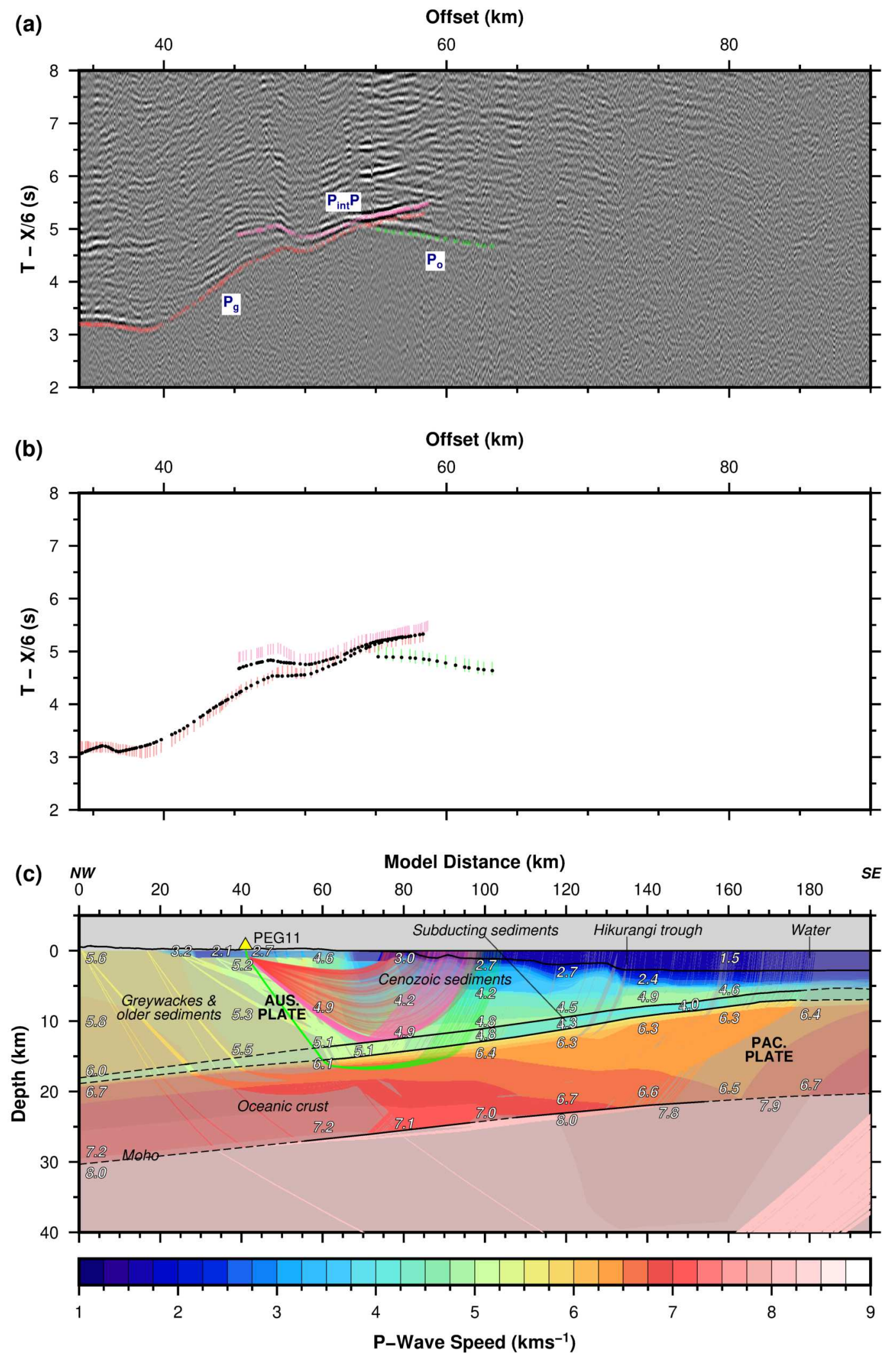

Figure A.9 (a) Common receiver gather at PEG11 station with airgun shots of PEGASUS23 line. Different seismic phases are colour-coded. $\mathrm{P}_{\mathrm{g}}$-red, $\mathrm{P}_{\text {int }} \mathrm{P}$-pink, $\mathrm{P}_{\mathrm{o}}$-light green, $\mathrm{P}_{\mathrm{m} 1} \mathrm{P}$-yellow and $\mathrm{P}_{\mathrm{n} 1}$-cyan. (b) Observed travel-times (colour-coded as in (a)) and calculated travel-times (black dots). The height of an observed travel-time pick is twice its pick uncertainty. Note that the travel-time axes of (a) and (b) are reduced at $6 \mathrm{~km} / \mathrm{s}$. (c) Traced rays and the velocity model, with rays colour-coded as in (a) and (b). Yellow triangle-location of seismograph. Grey shading indicates areas in the velocity model without ray coverage. White numbers with black outline are P-wave-speeds at those locations in $\mathrm{km} / \mathrm{s}$. Dashed black lines are inferred interfaces. Continuous black lines are confirmed interfaces. The x-axes in (a) and (b) indicate the offset from the recording station and the $x$-axis in (c) indicates the model distance starting from MRZ station. AUS. PLATE and PAC. PLATE are Australian Plate and Pacific Plate respectively. 
(a)

Offset (km)

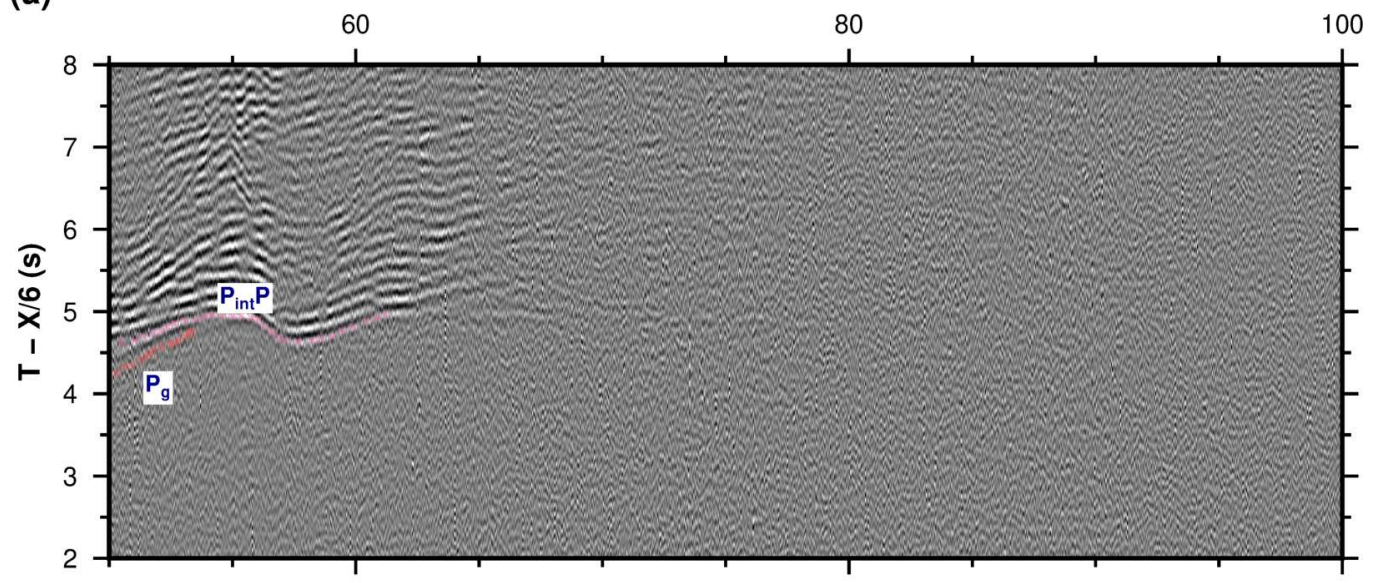

(b)

Offset $(\mathrm{km})$
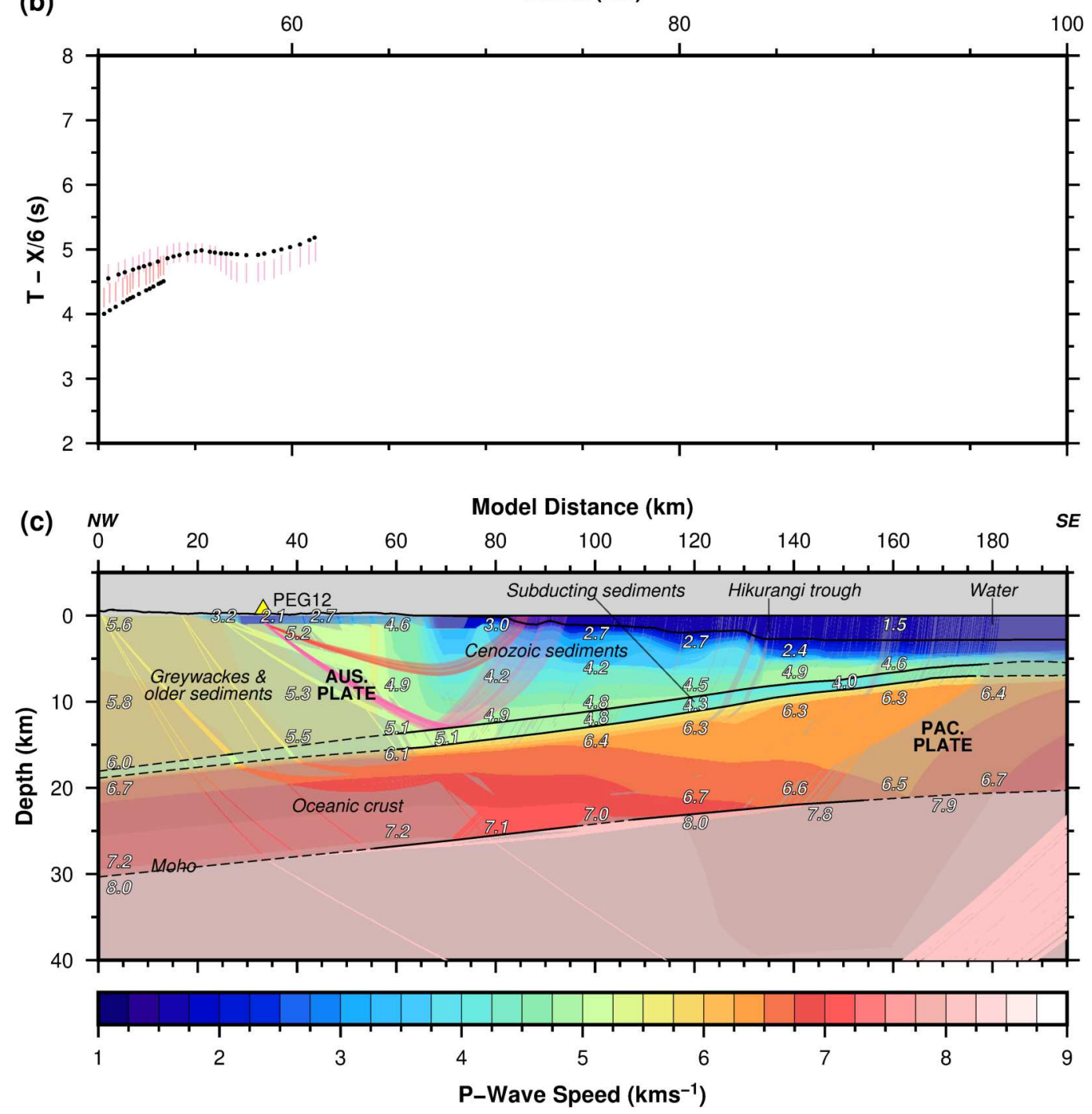

Figure A.10 (a) Common receiver gather at PEG12 station with airgun shots of PEGASUS23 line. Different seismic phases are colour-coded. $\mathrm{P}_{\mathrm{g}}$-red, $\mathrm{P}_{\text {int }} \mathrm{P}$-pink, $\mathrm{P}_{\mathrm{o}}$-light green, $\mathrm{P}_{\mathrm{m} 1} \mathrm{P}$-yellow and $\mathrm{P}_{\mathrm{n} 1}$-cyan. (b) Observed travel-times (colour-coded as in (a)) and calculated travel-times (black dots). The height of an observed travel-time pick is twice its pick uncertainty. Note that the travel-time axes of (a) and (b) are reduced at $6 \mathrm{~km} / \mathrm{s}$. (c) Traced rays and the velocity model, with rays colour-coded as in (a) and (b). Yellow triangle-location of seismograph. Grey shading indicates areas in the velocity model without ray coverage. White numbers with black outline are P-wave-speeds at those locations in $\mathrm{km} / \mathrm{s}$. Dashed black lines are inferred interfaces. Continuous black lines are confirmed interfaces. The x-axes in (a) and (b) indicate the offset from the recording station and the x-axis in (c) indicates the model distance starting from MRZ station. AUS. PLATE and PAC. PLATE are Australian Plate and Pacific Plate respectively. 
(a)

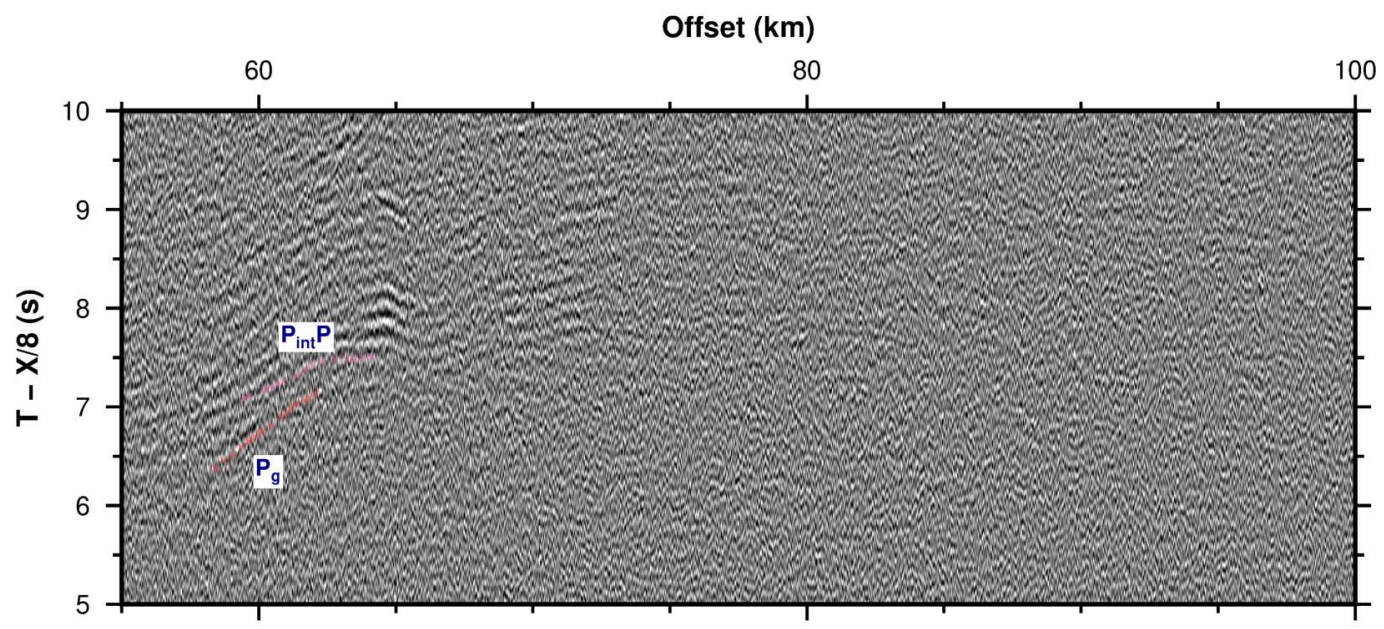

(b)

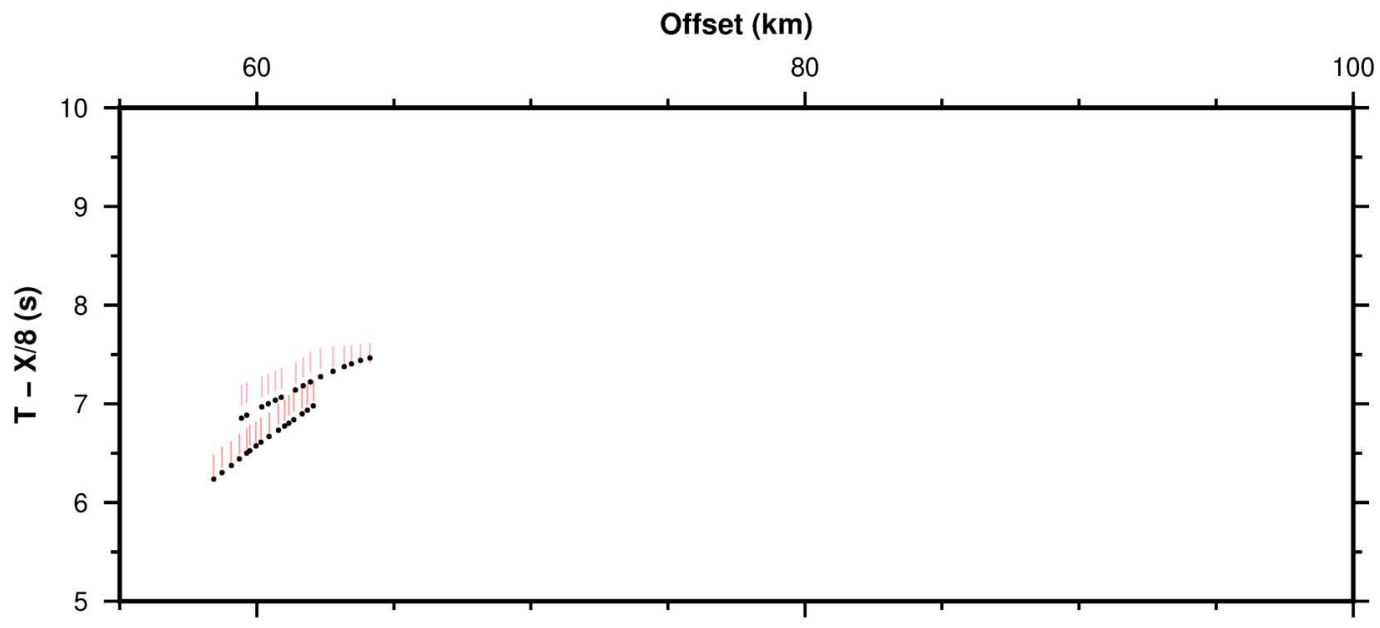

(c)

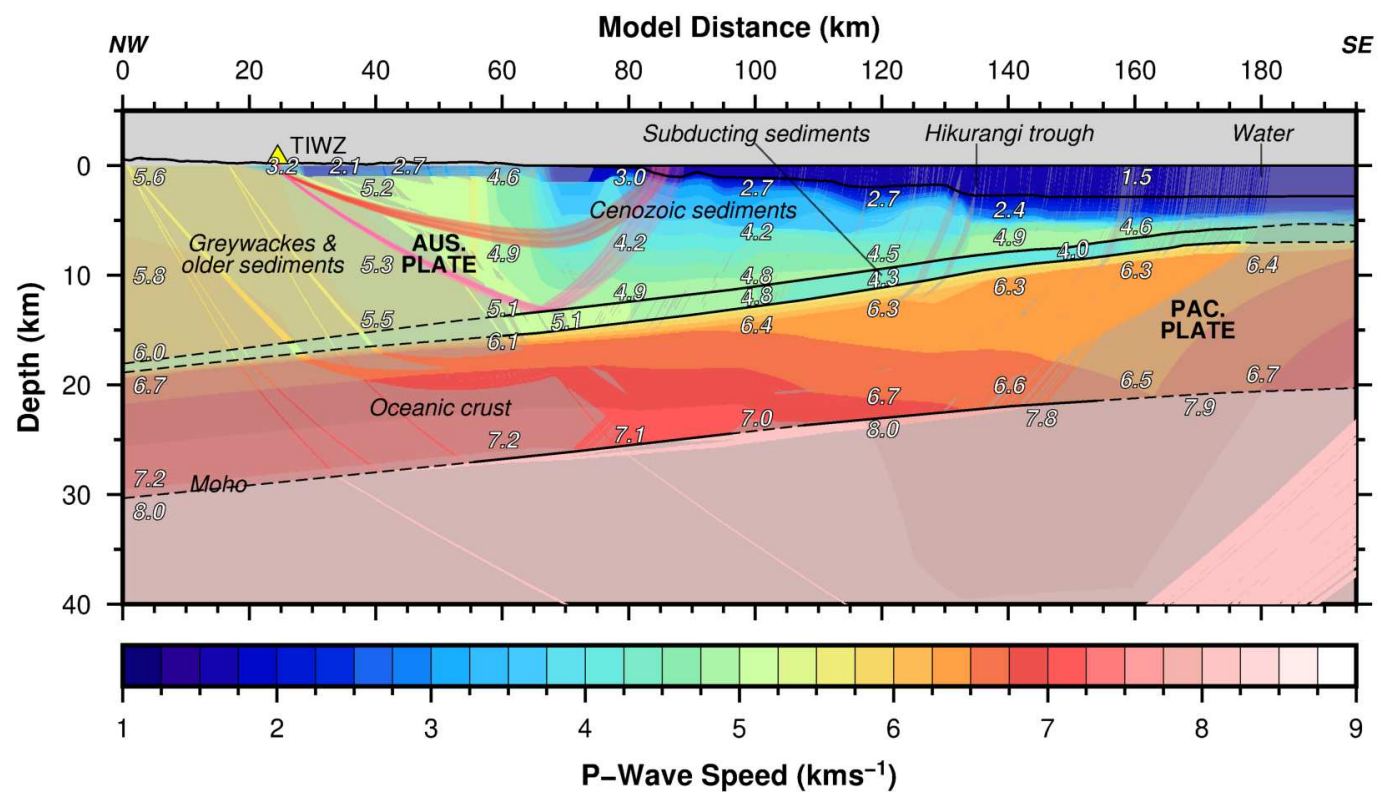

Figure A.11 (a) Common receiver gather at TIWZ station with airgun shots of PEGASUS23 line. Different seismic phases are colour-coded. $\mathrm{P}_{\mathrm{g}}$-red, $\mathrm{P}_{\text {int }} \mathrm{P}$-pink, $\mathrm{P}_{\mathrm{o}}$-light green, $\mathrm{P}_{\mathrm{m} 1} \mathrm{P}$-yellow and $\mathrm{P}_{\mathrm{n} 1}$-cyan. (b) Observed travel-times (colour-coded as in (a)) and calculated travel-times (black dots). The height of an observed travel-time pick is twice its pick uncertainty. Note that the travel-time axes of (a) and (b) are reduced at $6 \mathrm{~km} / \mathrm{s}$. (c) Traced rays and the velocity model, with rays colour-coded as in (a) and (b). Yellow triangle-location of seismograph. Grey shading indicates areas in the velocity model without ray coverage. White numbers with black outline are P-wave-speeds at those locations in $\mathrm{km} / \mathrm{s}$. Dashed black lines are inferred interfaces. Continuous black lines are confirmed interfaces. The x-axes in (a) and (b) indicate the offset from the recording station and the $\mathrm{x}$-axis in (c) indicates the model distance starting from MRZ station. AUS. PLATE and PAC. PLATE are Australian Plate and Pacific Plate respectively. 
(a)

Offset (km)

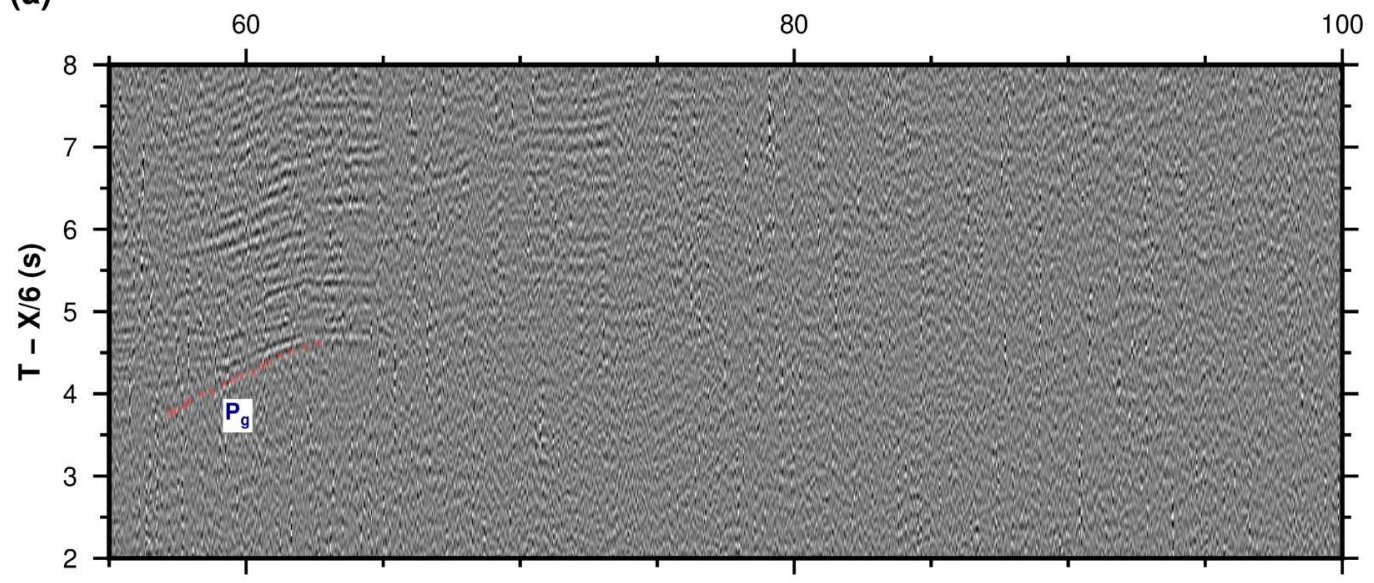

(b)

Offset (km)
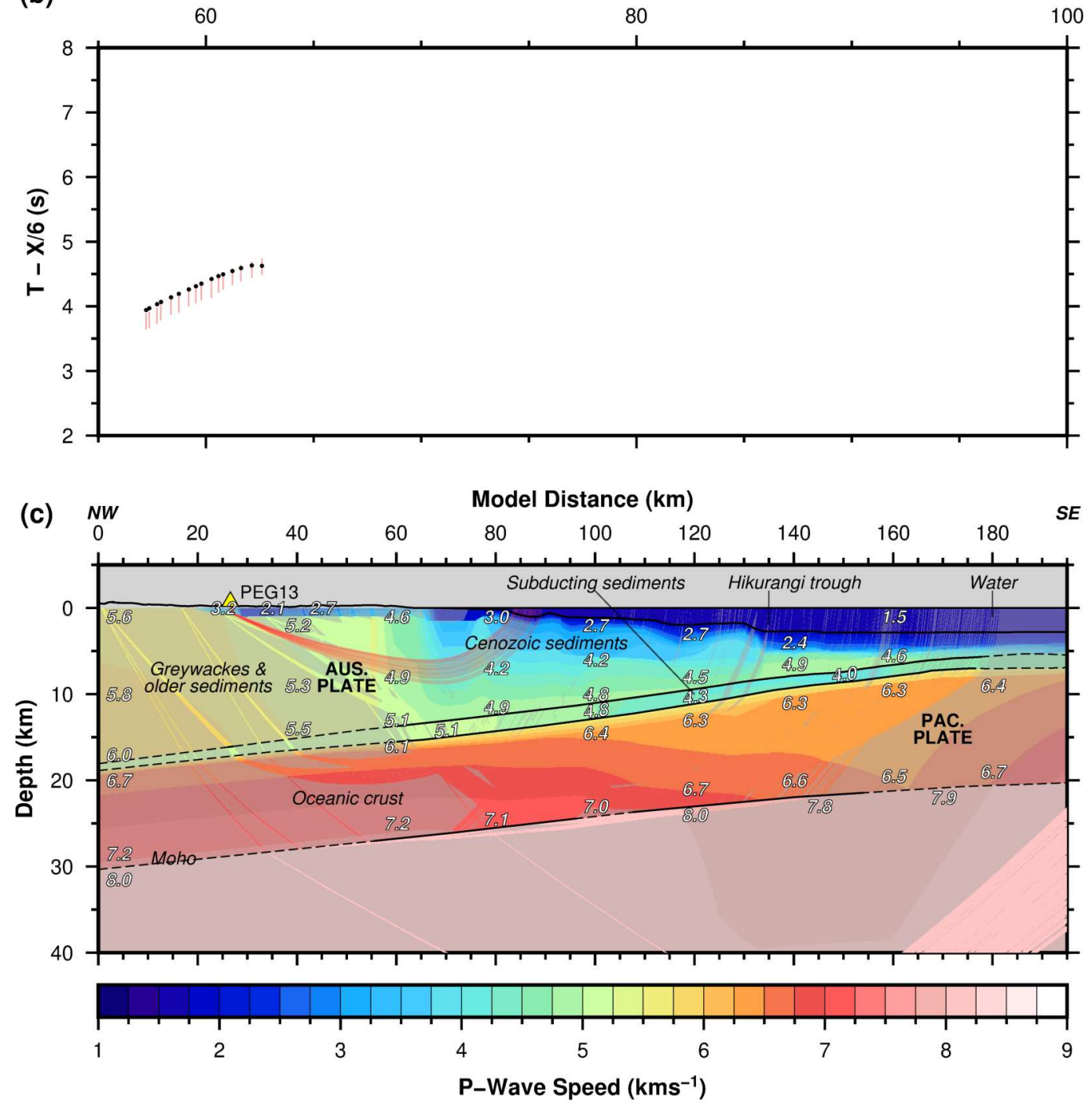

Figure A.12 (a) Common receiver gather at PEG13 station with airgun shots of PEGASUS23 line. Different seismic phases are colour-coded. $\mathrm{P}_{\mathrm{g}}$-red, $\mathrm{P}_{\text {int }} \mathrm{P}$-pink, $\mathrm{P}_{\mathrm{o}}$-light green, $\mathrm{P}_{\mathrm{m} 1} \mathrm{P}$-yellow and $\mathrm{P}_{\mathrm{n} 1}$-cyan. (b) Observed travel-times (colour-coded as in (a)) and calculated travel-times (black dots). The height of an observed travel-time pick is twice its pick uncertainty. Note that the travel-time axes of (a) and (b) are reduced at $6 \mathrm{~km} / \mathrm{s}$. (c) Traced rays and the velocity model, with rays colour-coded as in (a) and (b). Yellow triangle-location of seismograph. Grey shading indicates areas in the velocity model without ray coverage. White numbers with black outline are P-wave-speeds at those locations in $\mathrm{km} / \mathrm{s}$. Dashed black lines are inferred interfaces. Continuous black lines are confirmed interfaces. The x-axes in (a) and (b) indicate the offset from the recording station and the x-axis in (c) indicates the model distance starting from MRZ station. AUS. PLATE and PAC. PLATE are Australian Plate and Pacific Plate respectively. 

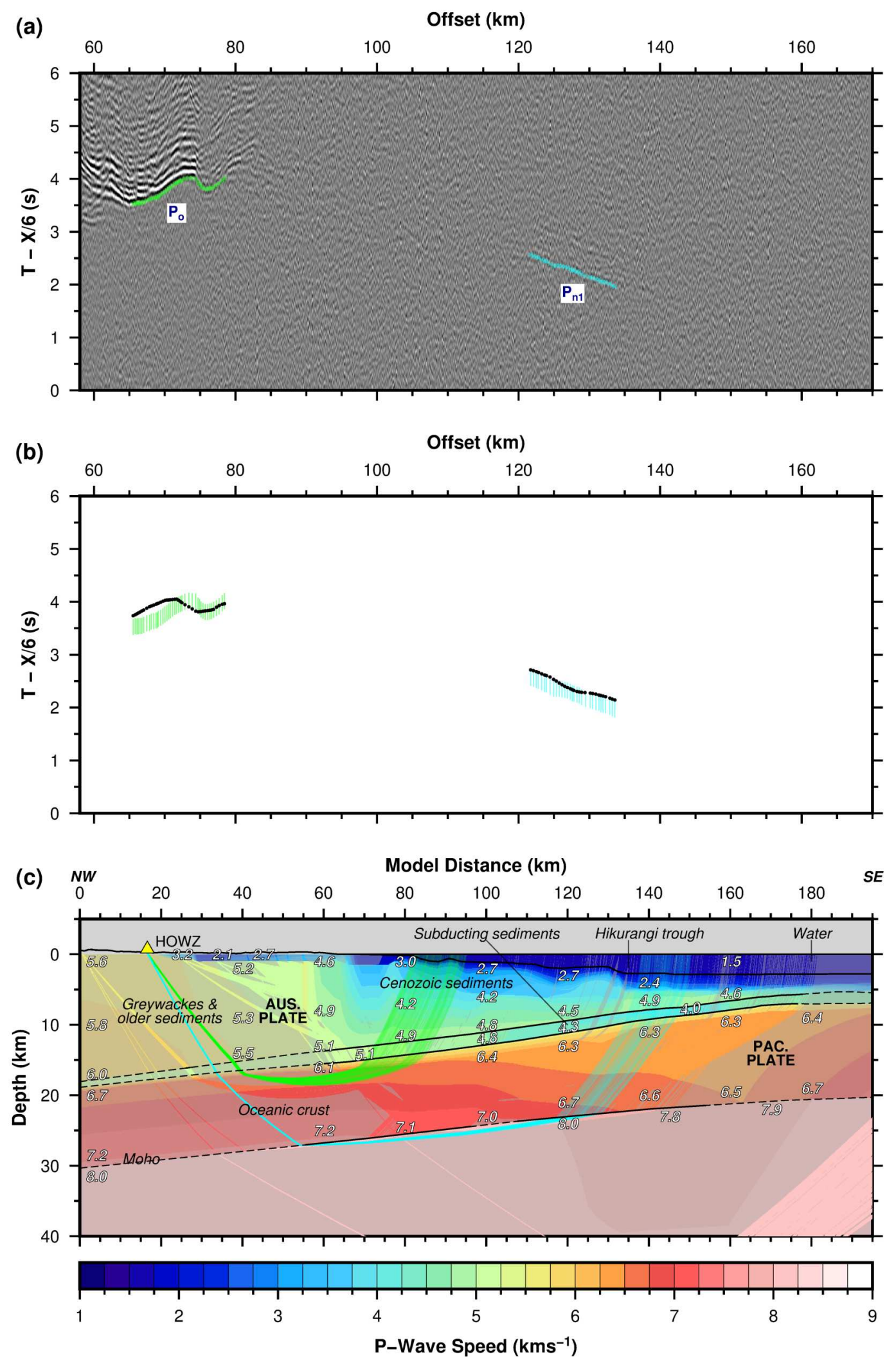

Figure A.13 (a) Common receiver gather at HOWZ station with airgun shots of PEGASUS23 line. Different seismic phases are colour-coded. $\mathrm{P}_{\mathrm{g}}$-red, $\mathrm{P}_{\text {int }} \mathrm{P}$-pink, $\mathrm{P}_{\mathrm{o}}$-light green, $\mathrm{P}_{\mathrm{m} 1} \mathrm{P}$-yellow and $\mathrm{P}_{\mathrm{n} 1}$-cyan. (b) Observed travel-times (colour-coded as in (a)) and calculated travel-times (black dots). The height of an observed travel-time pick is twice its pick uncertainty. Note that the travel-time axes of (a) and (b) are reduced at $6 \mathrm{~km} / \mathrm{s}$. (c) Traced rays and the velocity model, with rays colour-coded as in (a) and (b). Yellow triangle-location of seismograph. Grey shading indicates areas in the velocity model without ray coverage. White numbers with black outline are P-wave-speeds at those locations in $\mathrm{km} / \mathrm{s}$. Dashed black lines are inferred interfaces. Continuous black lines are confirmed interfaces. The x-axes in (a) and (b) indicate the offset from the recording station and the $x$-axis in (c) indicates the model distance starting from MRZ station. AUS. PLATE and PAC. PLATE are Australian Plate and Pacific Plate respectively. 
(a)

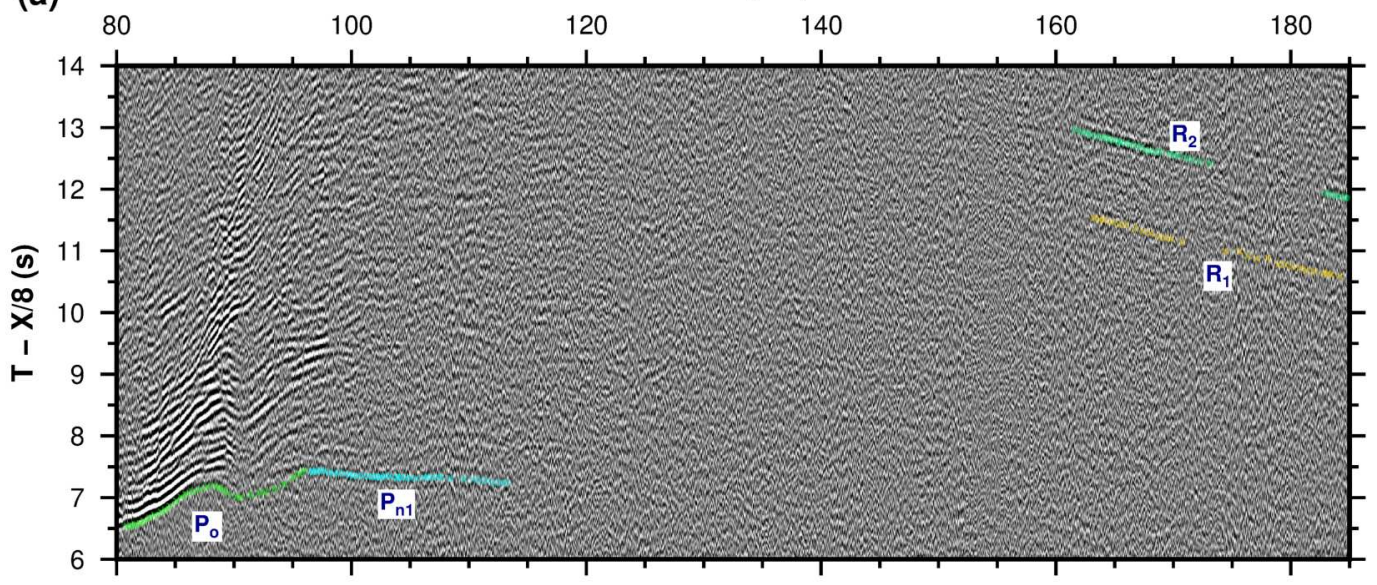

(b)
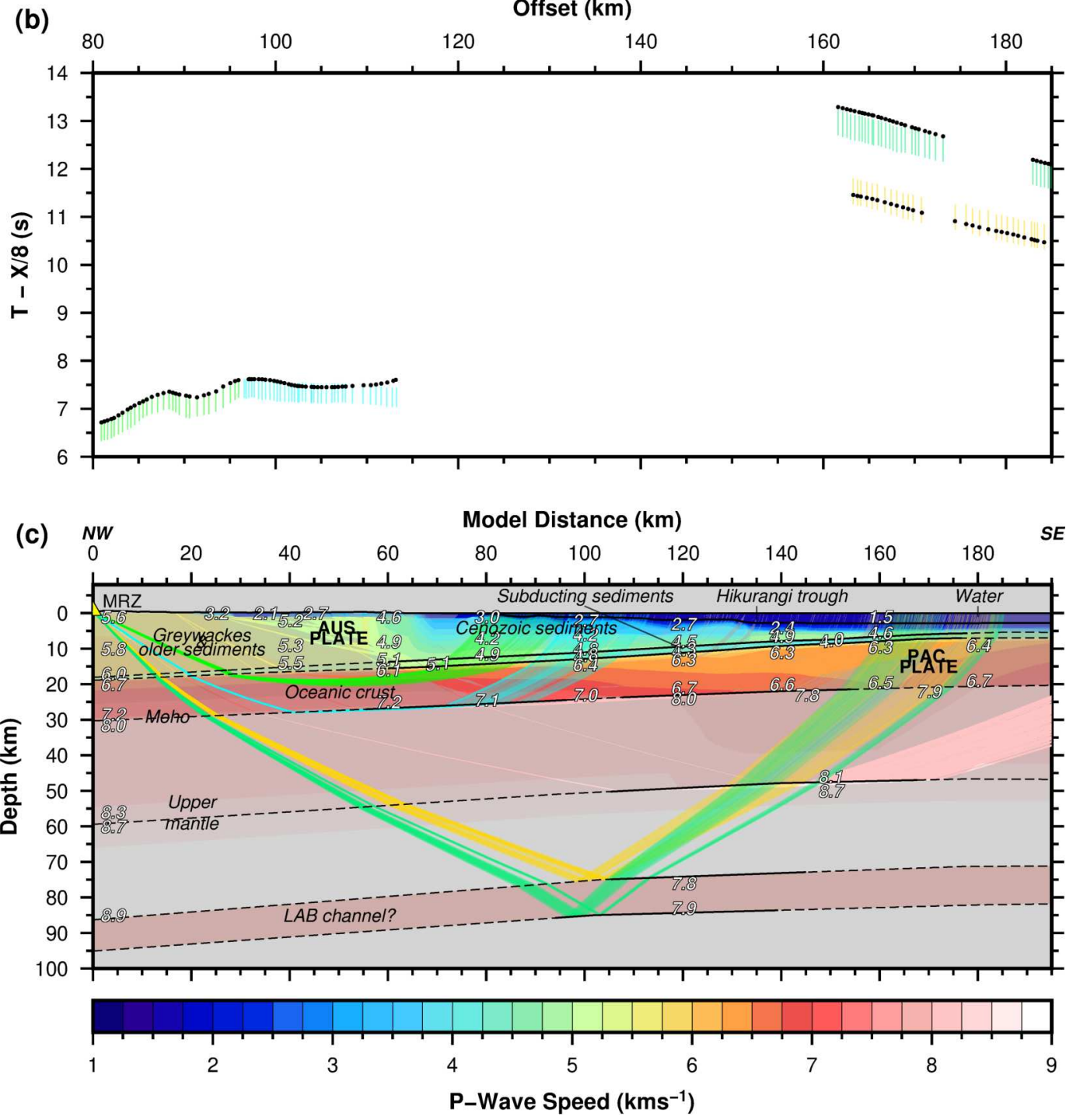

Figure A.14 (a) Common receiver gather at MRZ station with airgun shots of PEGASUS23 line. Different seismic phases are colour-coded. $\mathrm{P}_{\mathrm{g}}$-red, $\mathrm{P}_{\text {int }} \mathrm{P}$-pink, $\mathrm{P}_{\mathrm{o}}$-light green, $\mathrm{P}_{\mathrm{m} 1} \mathrm{P}$-yellow and $\mathrm{P}_{\mathrm{n} 1}$-cyan. (b) Observed travel-times (colour-coded as in (a)) and calculated travel-times (black dots). The height of an observed travel-time pick is twice its pick uncertainty. Note that the travel-time axes of (a) and (b) are reduced at $6 \mathrm{~km} / \mathrm{s}$. (c) Traced rays and the velocity model, with rays colour-coded as in (a) and (b). Yellow triangle-location of seismograph. Grey shading indicates areas in the velocity model without ray coverage. White numbers with black outline are P-wave-speeds at those locations in $\mathrm{km} / \mathrm{s}$. Dashed black lines are inferred interfaces. Continuous black lines are confirmed interfaces. The x-axes in (a) and (b) indicate the offset from the recording station and the x-axis in (c) indicates the model distance starting from MRZ station. AUS. PLATE and PAC. PLATE are Australian Plate and Pacific Plate respectively. 

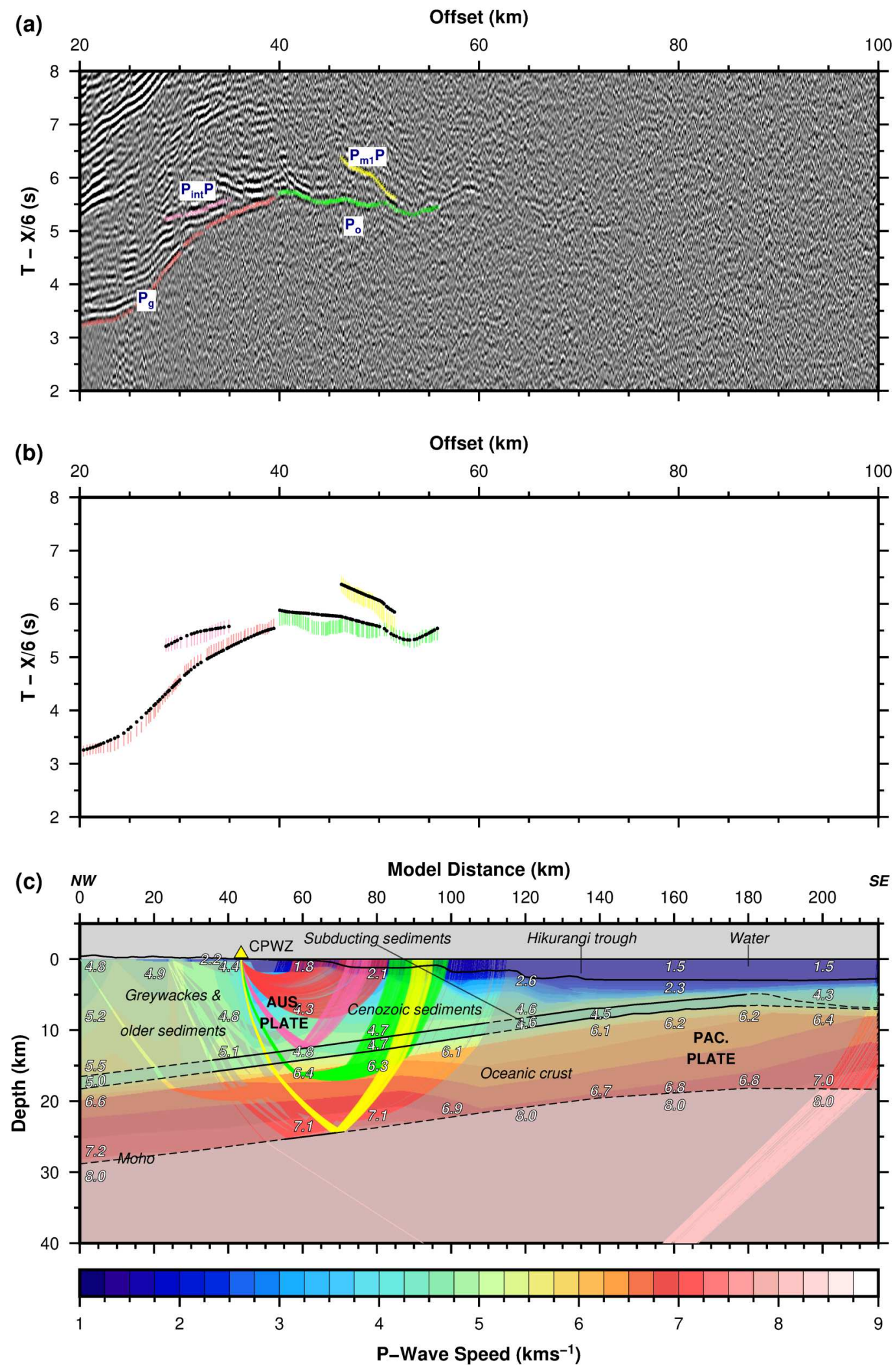

Figure A.15 (a) Common receiver gather at CPWZ station with airgun shots of PEGASUS25 line. Different seismic phases are colour-coded. $\mathrm{P}_{\mathrm{g}}$-red, $\mathrm{P}_{\text {int }} \mathrm{P}$-pink, $\mathrm{P}_{\mathrm{o}}$-light green, $\mathrm{P}_{\mathrm{m} 1} \mathrm{P}$-yellow and $\mathrm{P}_{\mathrm{n} 1}$-cyan. (b) Observed travel-times (colour-coded as in (a)) and calculated travel-times (black dots). The height of an observed travel-time pick is twice its pick uncertainty. Note that the travel-time axes of (a) and (b) are reduced at $6 \mathrm{~km} / \mathrm{s}$. (c) Traced rays and the velocity model, with rays colour-coded as in (a) and (b). Yellow triangle-location of seismograph. Grey shading indicates areas in the velocity model without ray coverage. White numbers with black outline are P-wave-speeds at those locations in $\mathrm{km} / \mathrm{s}$. Dashed black lines are inferred interfaces. Continuous black lines are confirmed interfaces. The x-axes in (a) and (b) indicate the offset from the recording station and the $x$-axis in (c) indicates the model distance starting from MRZ station. AUS. PLATE and PAC. PLATE are Australian Plate and Pacific Plate respectively. 
(a)
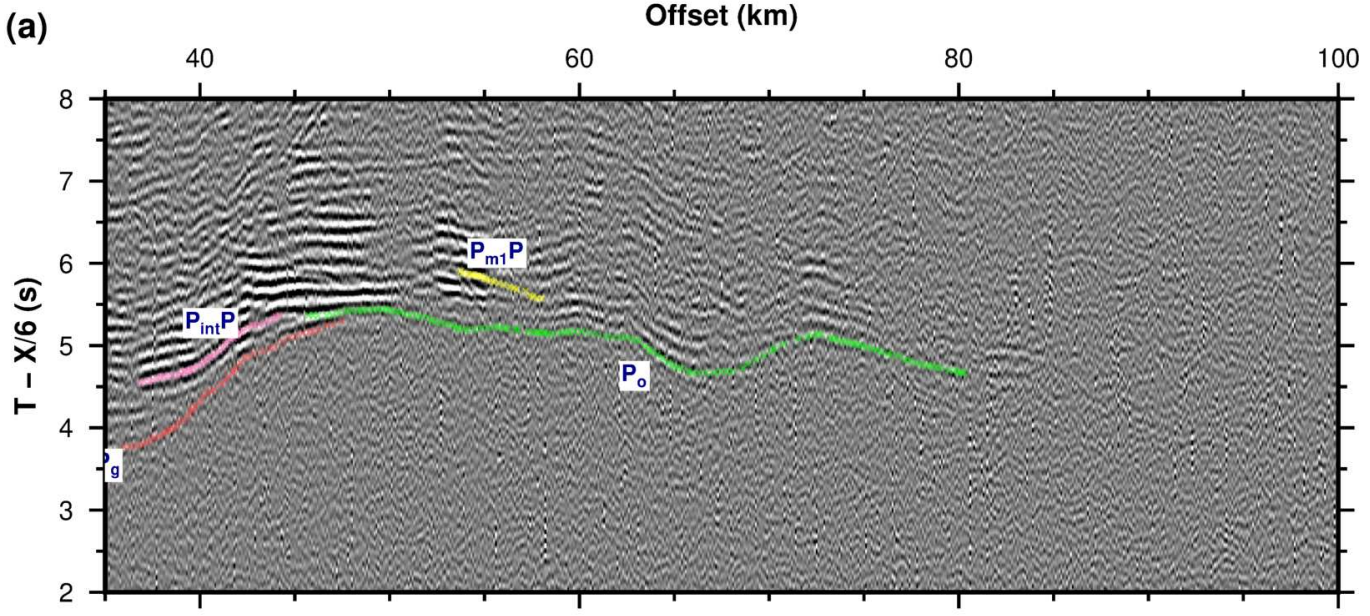

(b)
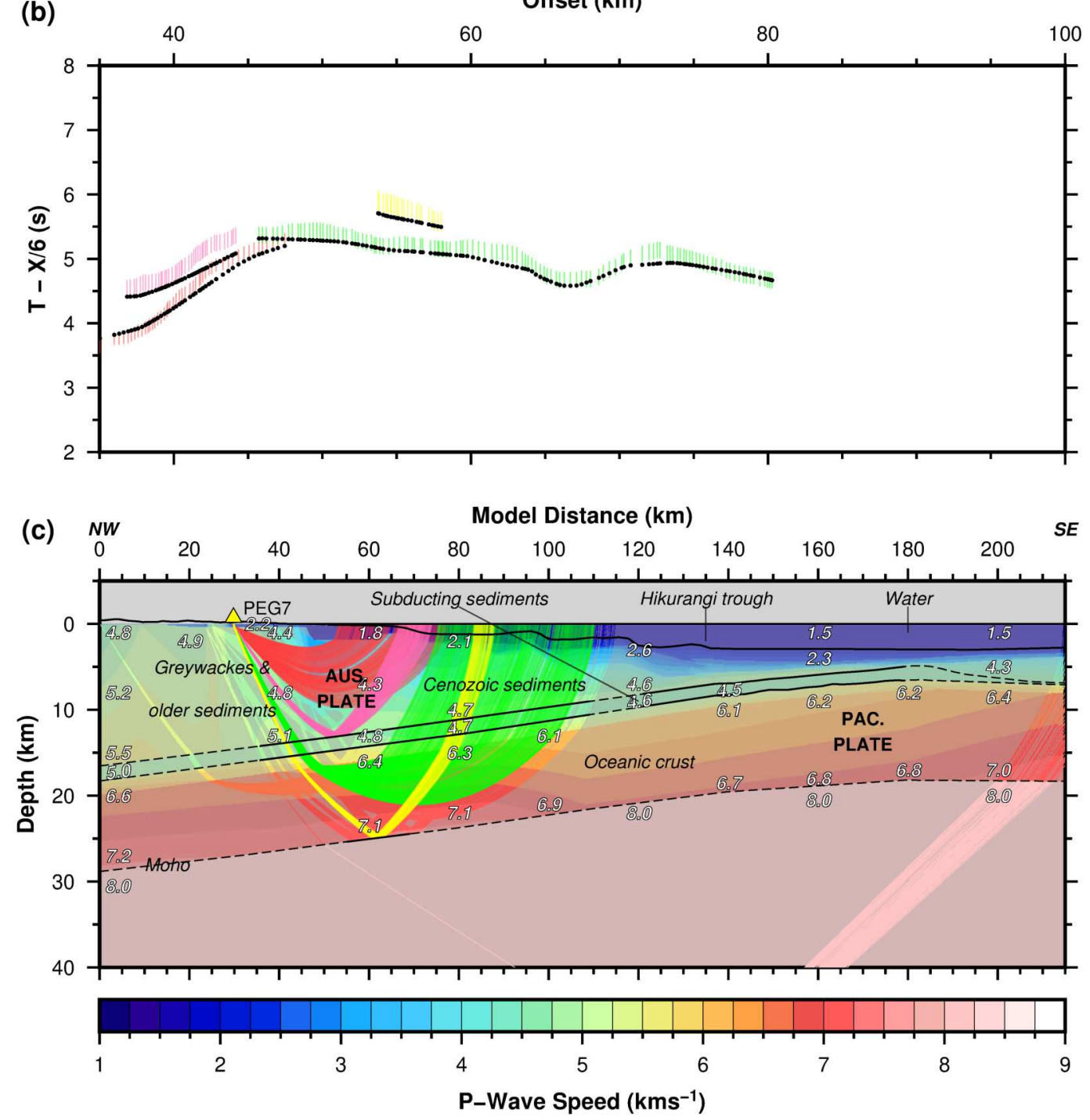

Figure A.16 (a) Common receiver gather at PEG7 station with airgun shots of PEGASUS25 line. Different seismic phases are colour-coded. $\mathrm{P}_{\mathrm{g}}$-red, $\mathrm{P}_{\text {int }} \mathrm{P}$-pink, $\mathrm{P}_{\mathrm{o}}$-light green, $\mathrm{P}_{\mathrm{m} 1} \mathrm{P}$-yellow and $\mathrm{P}_{\mathrm{n} 1}$-cyan. (b) Observed travel-times (colour-coded as in (a)) and calculated travel-times (black dots). The height of an observed travel-time pick is twice its pick uncertainty. Note that the travel-time axes of (a) and (b) are reduced at $6 \mathrm{~km} / \mathrm{s}$. (c) Traced rays and the velocity model, with rays colour-coded as in (a) and (b). Yellow triangle-location of seismograph. Grey shading indicates areas in the velocity model without ray coverage. White numbers with black outline are P-wave-speeds at those locations in $\mathrm{km} / \mathrm{s}$. Dashed black lines are inferred interfaces. Continuous black lines are confirmed interfaces. The x-axes in (a) and (b) indicate the offset from the recording station and the x-axis in (c) indicates the model distance starting from MRZ station. AUS. PLATE and PAC. PLATE are Australian Plate and Pacific Plate respectively. 

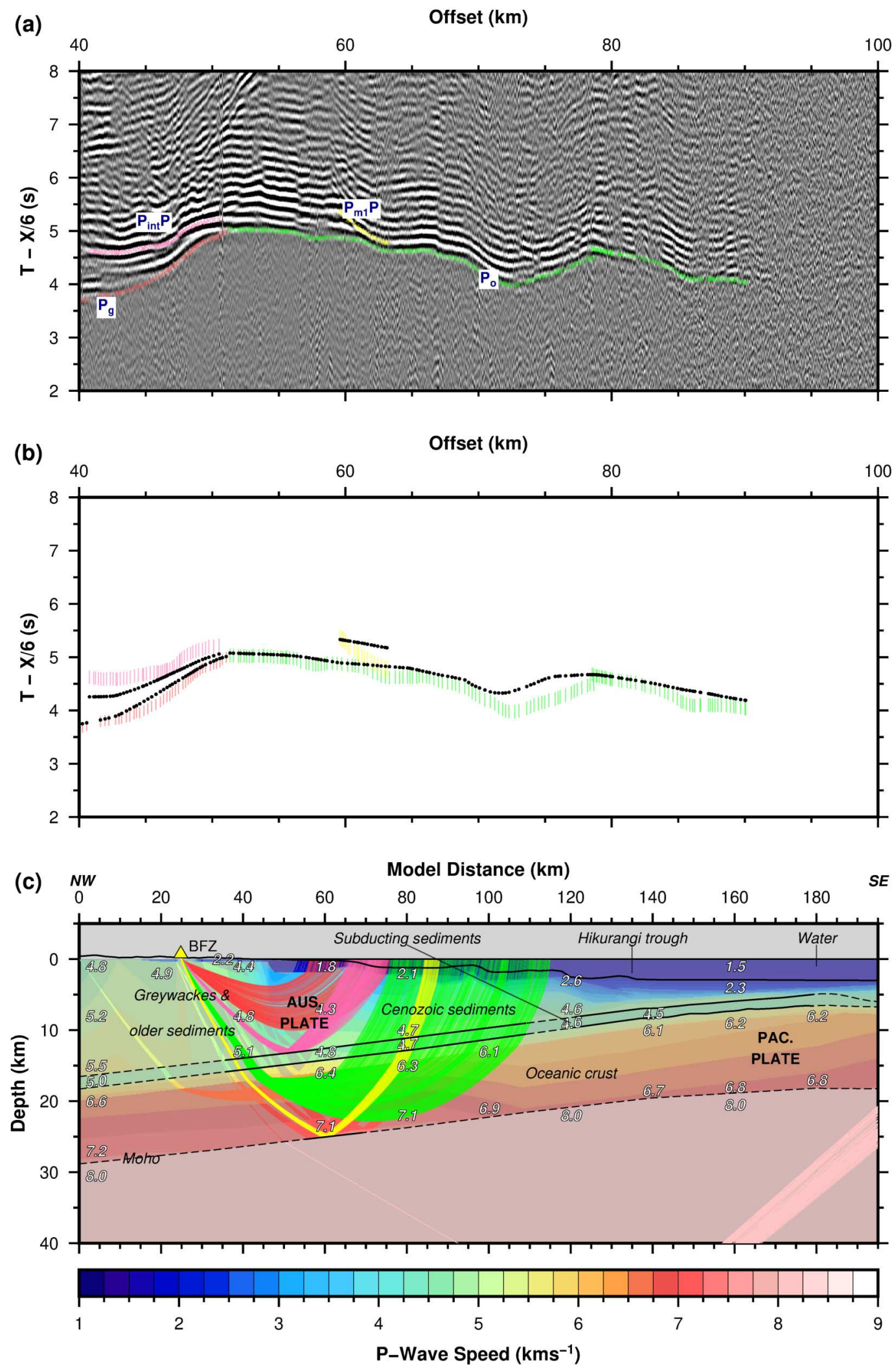

Figure A.17 (a) Common receiver gather at BFZ station with airgun shots of PEGASUS25 line. Different seismic phases are colour-coded. $\mathrm{P}_{\mathrm{g}}$-red, $\mathrm{P}_{\text {int }} \mathrm{P}$-pink, $\mathrm{P}_{\mathrm{o}}$-light green, $\mathrm{P}_{\mathrm{m} 1} \mathrm{P}$-yellow and $\mathrm{P}_{\mathrm{n} 1}$-cyan. (b) Observed travel-times (colour-coded as in (a)) and calculated travel-times (black dots). The height of an observed travel-time pick is twice its pick uncertainty. Note that the travel-time axes of (a) and (b) are reduced at $6 \mathrm{~km} / \mathrm{s}$. (c) Traced rays and the velocity model, with rays colour-coded as in (a) and (b). Yellow triangle-location of seismograph. Grey shading indicates areas in the velocity model without ray coverage. White numbers with black outline are P-wave-speeds at those locations in $\mathrm{km} / \mathrm{s}$. Dashed black lines are inferred interfaces. Continuous black lines are confirmed interfaces. The x-axes in (a) and (b) indicate the offset from the recording station and the $x$-axis in (c) indicates the model distance starting from MRZ station. AUS. PLATE and PAC. PLATE are Australian Plate and Pacific Plate respectively. 
(a)

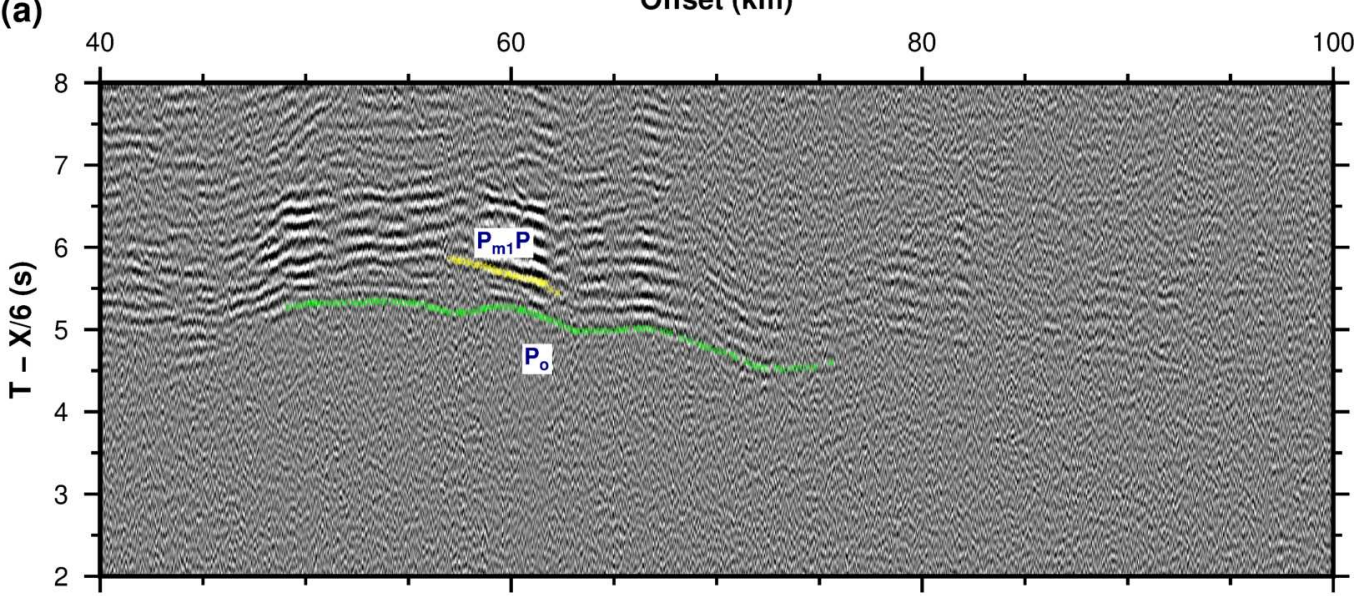

(b)

Offset (km)
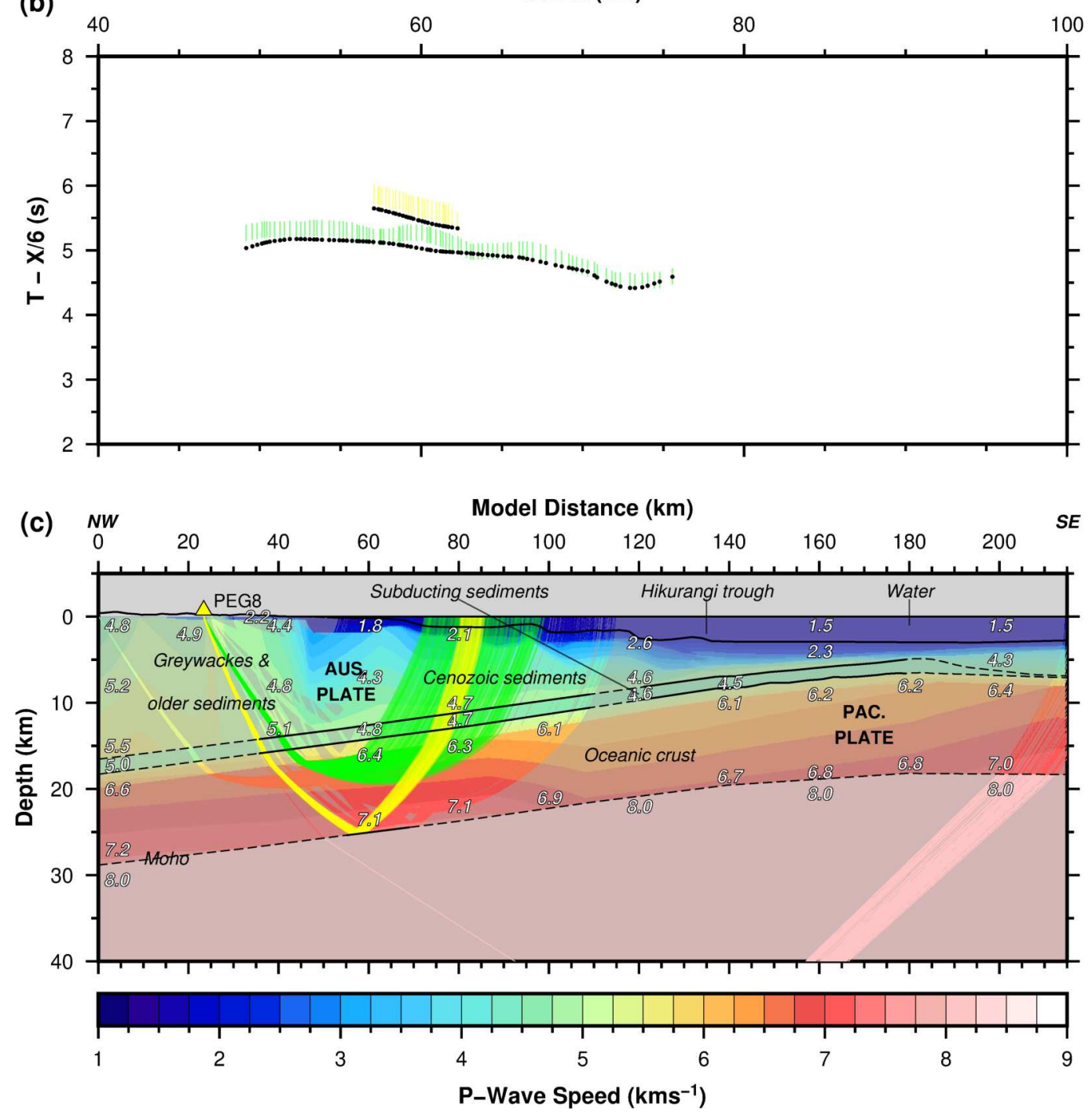

Figure A.18 (a) Common receiver gather at PEG8 station with airgun shots of PEGASUS25 line. Different seismic phases are colour-coded. $\mathrm{P}_{\mathrm{g}}$-red, $\mathrm{P}_{\text {int }} \mathrm{P}$-pink, $\mathrm{P}_{\mathrm{o}}$-light green, $\mathrm{P}_{\mathrm{m} 1} \mathrm{P}$-yellow and $\mathrm{P}_{\mathrm{n} 1}$-cyan. (b) Observed travel-times (colour-coded as in (a)) and calculated travel-times (black dots). The height of an observed travel-time pick is twice its pick uncertainty. Note that the travel-time axes of (a) and (b) are reduced at $6 \mathrm{~km} / \mathrm{s}$. (c) Traced rays and the velocity model, with rays colour-coded as in (a) and (b). Yellow triangle-location of seismograph. Grey shading indicates areas in the velocity model without ray coverage. White numbers with black outline are P-wave-speeds at those locations in $\mathrm{km} / \mathrm{s}$. Dashed black lines are inferred interfaces. Continuous black lines are confirmed interfaces. The x-axes in (a) and (b) indicate the offset from the recording station and the x-axis in (c) indicates the model distance starting from MRZ station. AUS. PLATE and PAC. PLATE are Australian Plate and Pacific Plate respectively. 
(a)

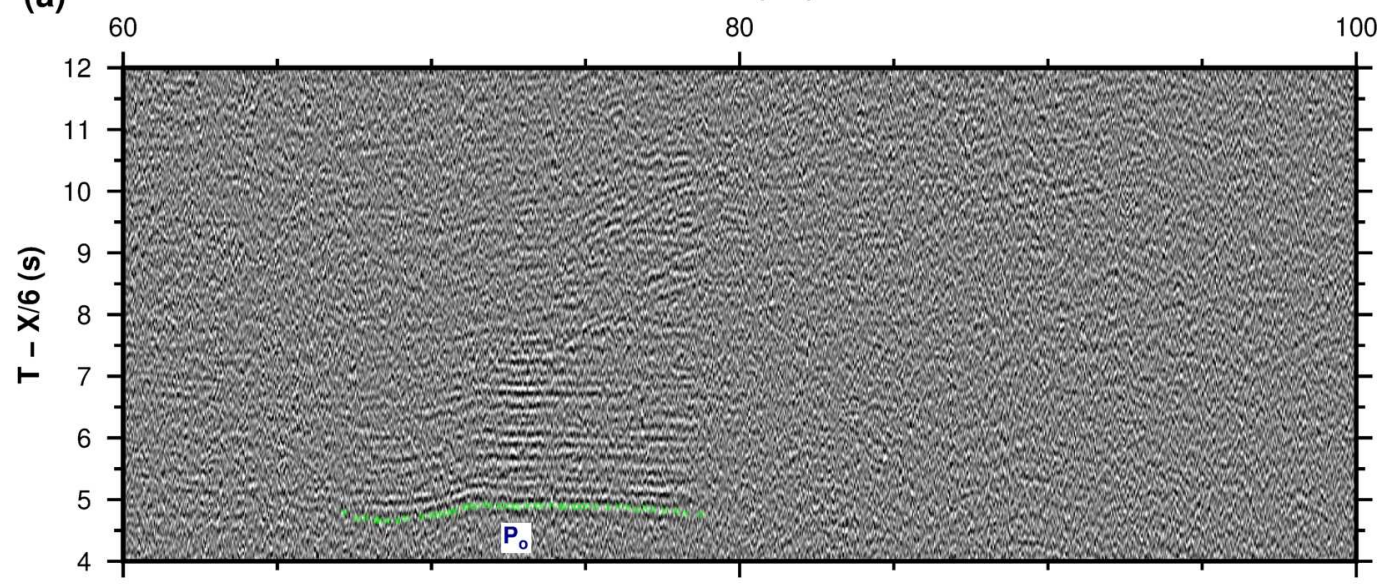

(b)
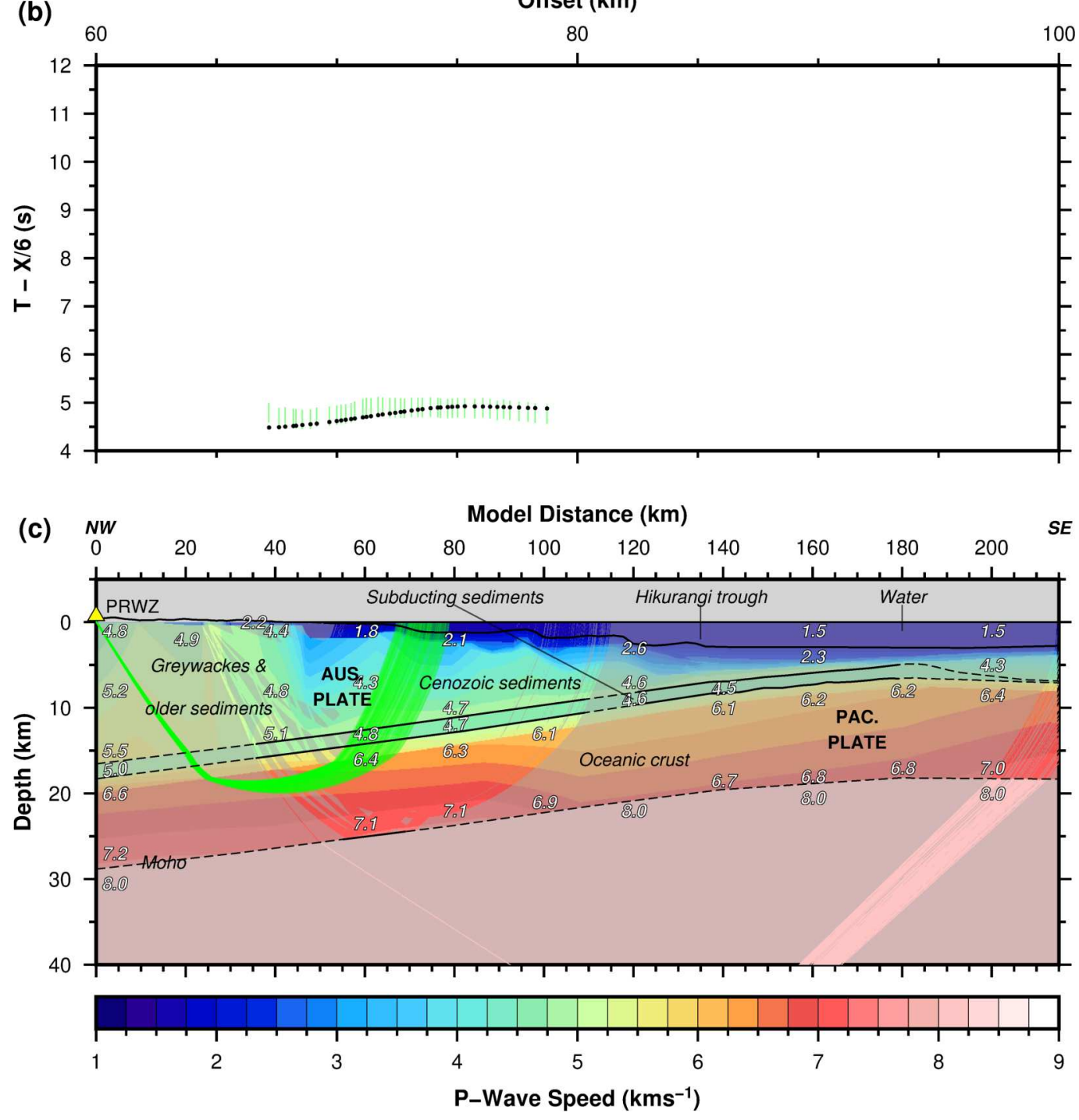

Figure A.19 (a) Common receiver gather at PRWZ station with airgun shots of PEGASUS25 line. Different seismic phases are colour-coded. $\mathrm{P}_{\mathrm{g}}$-red, $\mathrm{P}_{\text {int }} \mathrm{P}$-pink, $\mathrm{P}_{\mathrm{o}}$-light green, $\mathrm{P}_{\mathrm{m} 1} \mathrm{P}$-yellow and $\mathrm{P}_{\mathrm{n} 1}$-cyan. (b) Observed travel-times (colour-coded as in (a)) and calculated travel-times (black dots). The height of an observed travel-time pick is twice its pick uncertainty. Note that the travel-time axes of (a) and (b) are reduced at $6 \mathrm{~km} / \mathrm{s}$. (c) Traced rays and the velocity model, with rays colour-coded as in (a) and (b). Yellow triangle-location of seismograph. Grey shading indicates areas in the velocity model without ray coverage. White numbers with black outline are P-wave-speeds at those locations in $\mathrm{km} / \mathrm{s}$. Dashed black lines are inferred interfaces. Continuous black lines are confirmed interfaces. The x-axes in (a) and (b) indicate the offset from the recording station and the $x$-axis in (c) indicates the model distance starting from MRZ station. AUS. PLATE and PAC. PLATE are Australian Plate and Pacific Plate respectively. 

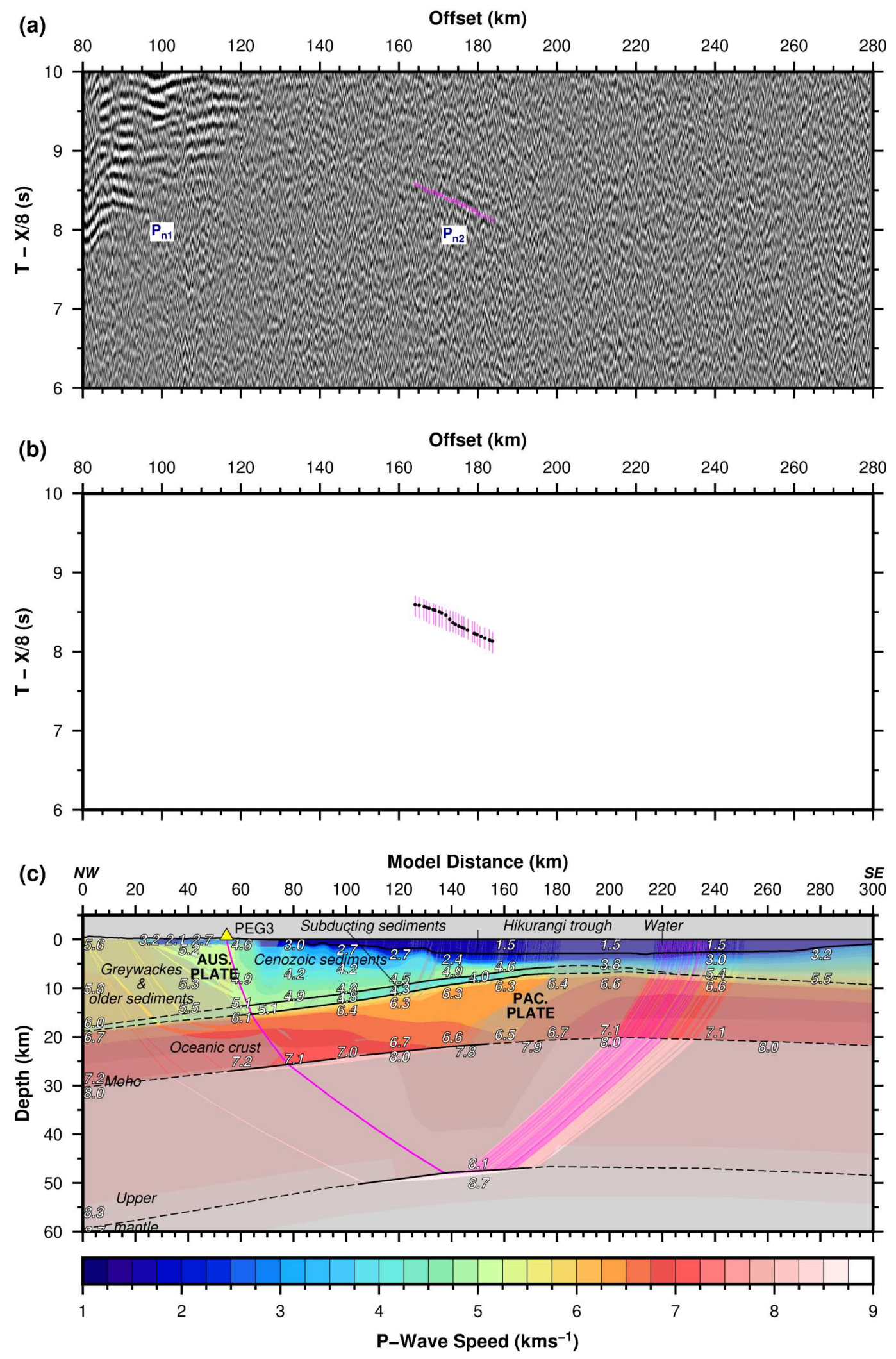

Figure A.20 (a) Common receiver gather at PEG3 station with airgun shots of SAHKE01 line. Different seismic phases are colour-coded. $\mathrm{P}_{\mathrm{g}}$-red, $\mathrm{P}_{\text {int }} \mathrm{P}$-pink, $\mathrm{P}_{\mathrm{o}}$-light green, $\mathrm{P}_{\mathrm{m} 1} \mathrm{P}$-yellow and $\mathrm{P}_{\mathrm{n} 1}$-cyan. (b) Observed travel-times (colour-coded as in (a)) and calculated travel-times (black dots). The height of an observed travel-time pick is twice its pick uncertainty. Note that the travel-time axes of (a) and (b) are reduced at $6 \mathrm{~km} / \mathrm{s}$. (c) Traced rays and the velocity model, with rays colour-coded as in (a) and (b). Yellow triangle-location of seismograph. Grey shading indicates areas in the velocity model without ray coverage. White numbers with black outline are P-wave-speeds at those locations in $\mathrm{km} / \mathrm{s}$. Dashed black lines are inferred interfaces. Continuous black lines are confirmed interfaces. The x-axes in (a) and (b) indicate the offset from the recording station and the $x$-axis in (c) indicates the model distance starting from MRZ station. AUS. PLATE and PAC. PLATE are Australian Plate and Pacific Plate respectively. 




Figure A.21 Multichannel seismic reflection image along PEGASUS23 line overlain by stacking velocities. White lines are the prominent reflectors. Black lines are velocity contours (contour interval is $0.5 \mathrm{~km} / \mathrm{s}$ ). $\mathrm{X}$-axis is the same model distance as in the transect.

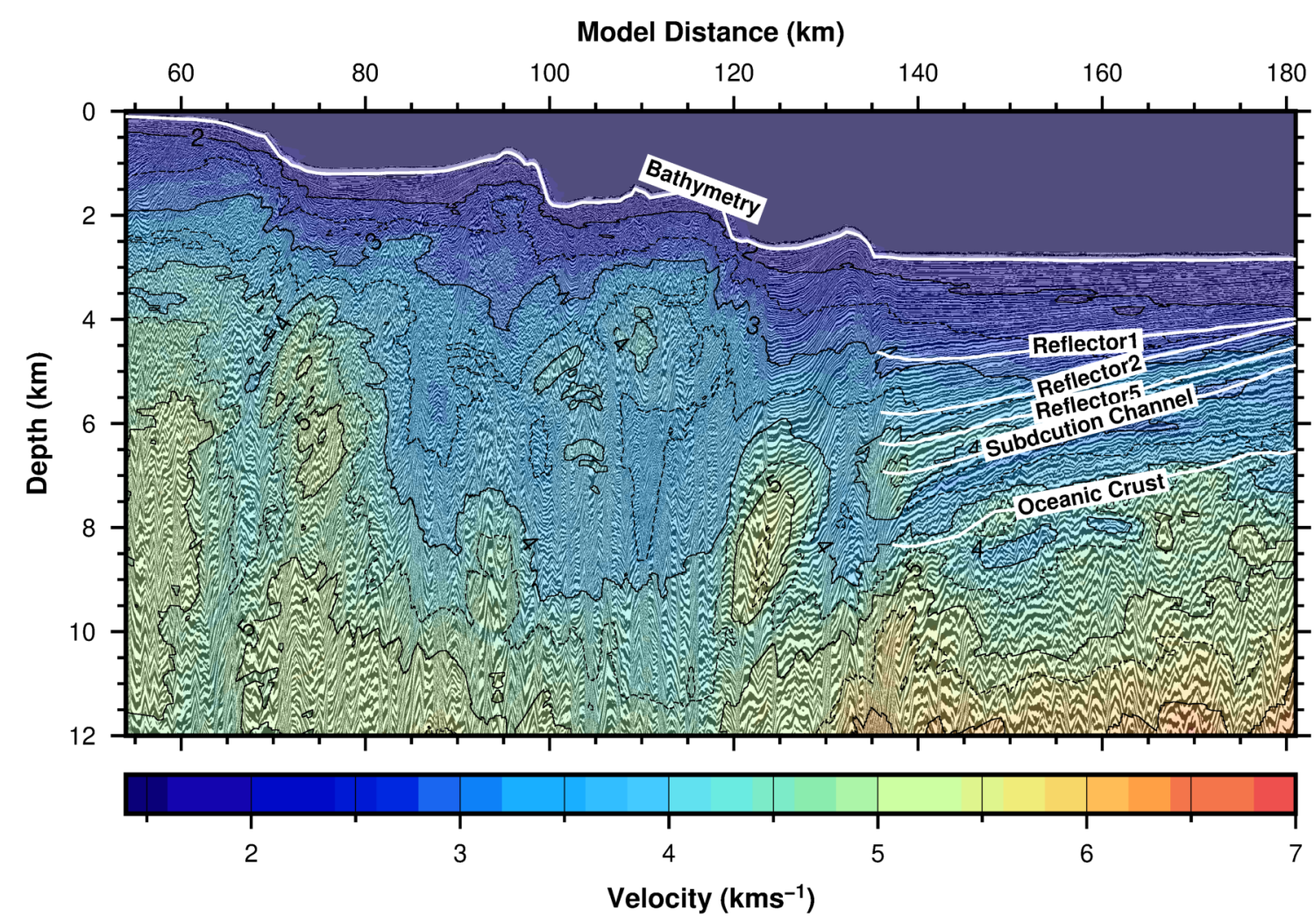

Figure A.22 Multichannel seismic reflection image along PEGASUS25 line overlain by stacking velocities. White lines are the prominent reflectors. Black lines are velocity contours (contour interval is $0.5 \mathrm{~km} / \mathrm{s}$ ). $\mathrm{X}$-axis is the same model distance as in the transect. 


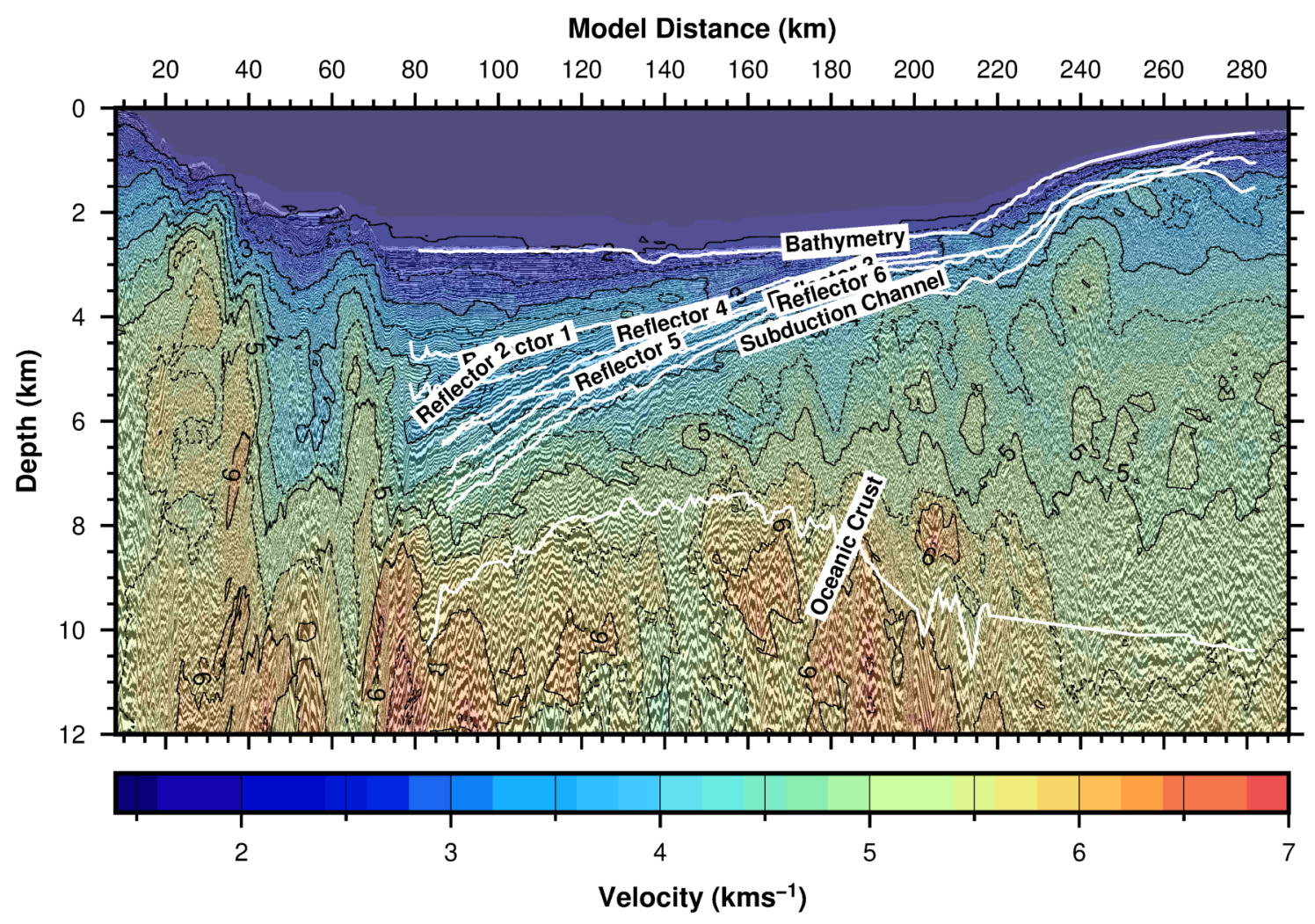

Figure A.23 Multichannel seismic reflection image along SAHKE01 line overlain by stacking velocities. White lines are the prominent reflectors. Black lines are velocity contours (contour interval is $0.5 \mathrm{~km} / \mathrm{s}$ ). Origin of the X-axis is at TRWZ station (see Figure 3.1a).
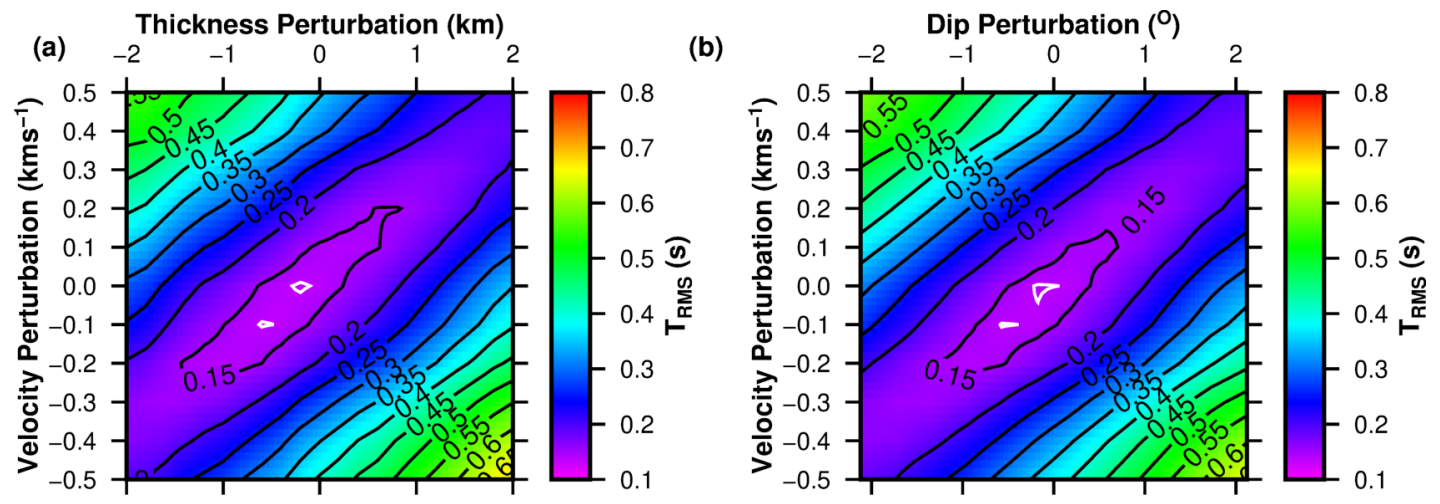

Figure A.24 (a) Perturbation test results for the uncertainties in the P-wave-speed at the base and thickness of the Australian Plate crust. (b) Perturbation test results for the uncertainty in the dip of the plate interface and the P-wave-speed at the base of the Australian Plate crust. White contour-95\% confidence level contour. 


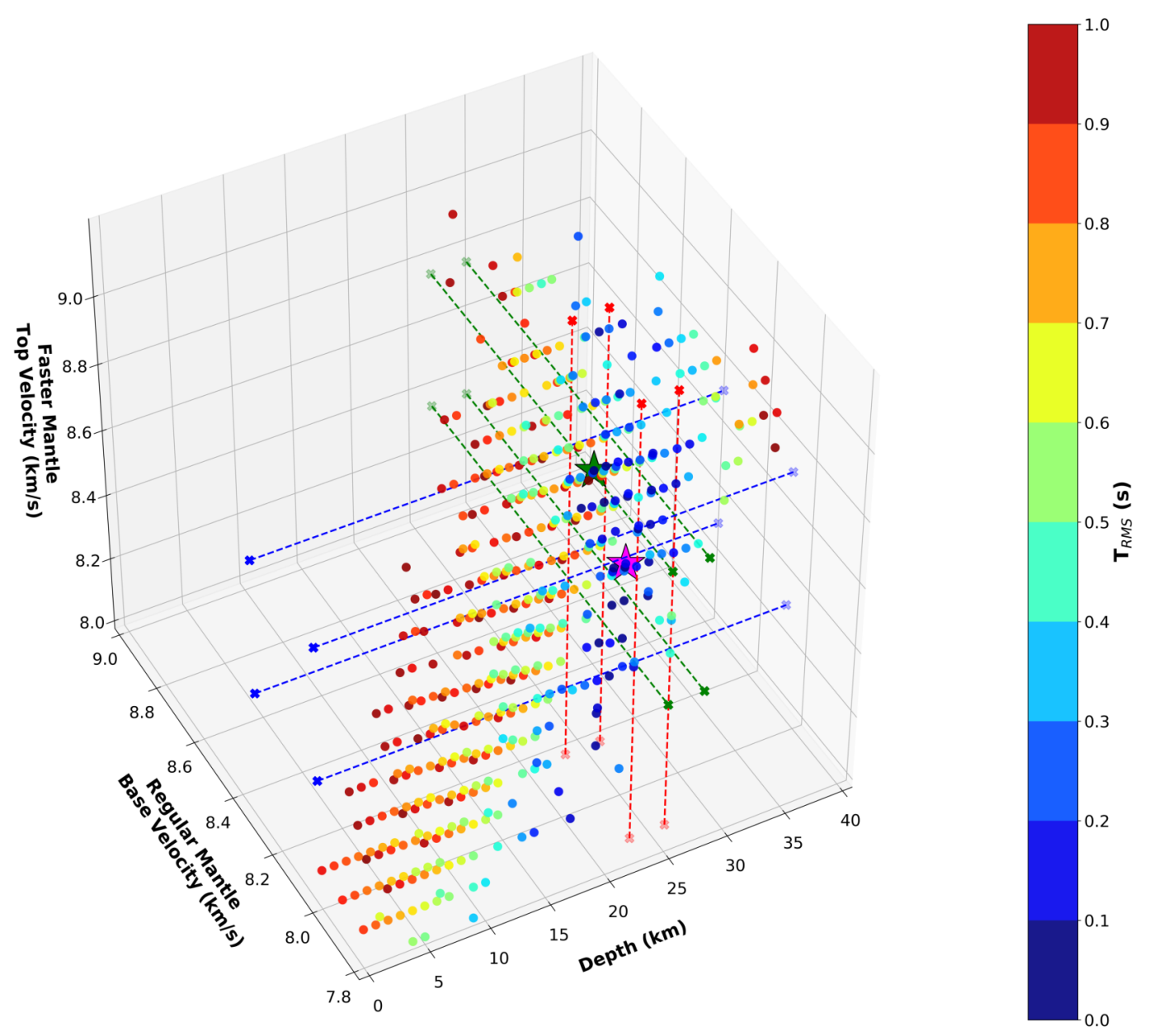

Figure A.25 Three-parameter perturbation test to estimate the uncertainties of the velocity at the base of the regular mantle, depth and velocity of the top of the faster mantle layer. $\mathrm{T}_{\mathrm{RMS}}$ for model parameter combinations that could trace more than $90 \%$ of observed travel-time picks of $P_{n 2}$ phase $(106 / 118$ points) with $\mathrm{T}_{\mathrm{RMS}}<1 \mathrm{sec}$ are plotted here. Dashed red, green and blue lines indicate the minimum and maximum trade-offs of regular mantle base velocity, depth to and velocity of the top of the faster upper mantle layer, respectively. Magenta and green stars indicate the preferred and minimum $T_{R M S}$ model parameter combinations, respectively. Note that both preferred and minimum $\mathrm{T}_{\mathrm{RMS}}$ model parameter combinations fall within the cuboid defined by the intersecting dashed lines. Corners of the cuboid are: $7.9 \mathrm{~km} / \mathrm{s}$ and $8.2 \mathrm{~km} / \mathrm{s}$ along the "regular mantle base velocity" axis; $8.5 \mathrm{~km} / \mathrm{s}$ and $8.9 \mathrm{~km} / \mathrm{s}$ along the "faster mantle top velocity" axis; and $24 \mathrm{~km}$ and $27 \mathrm{~km}$ along the "depth" axis.

(a)

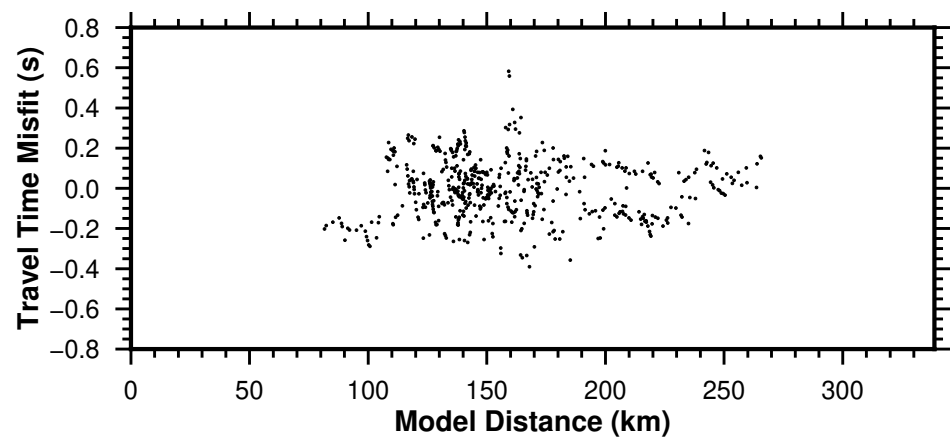

(b)

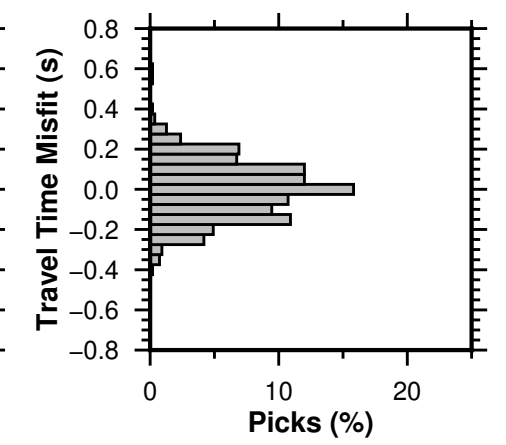

Figure A.26 (a) Plot of travel-time misfits with model distance along PEGASUS23 transect. (b) Histogram of travel-time misfits binned every $50 \mathrm{msec}$. 
(a)

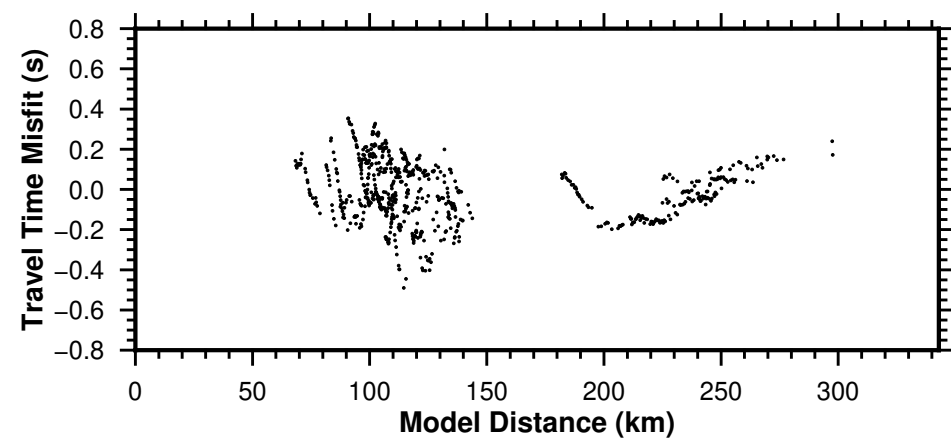

(b)

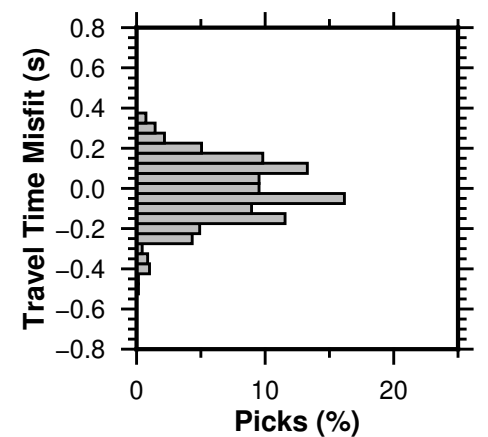

Figure A.27 (a) Plot of travel-time misfits with model distance along PEGASUS25 transect. (b) Histogram of travel-time misfits binned every $50 \mathrm{msec}$.

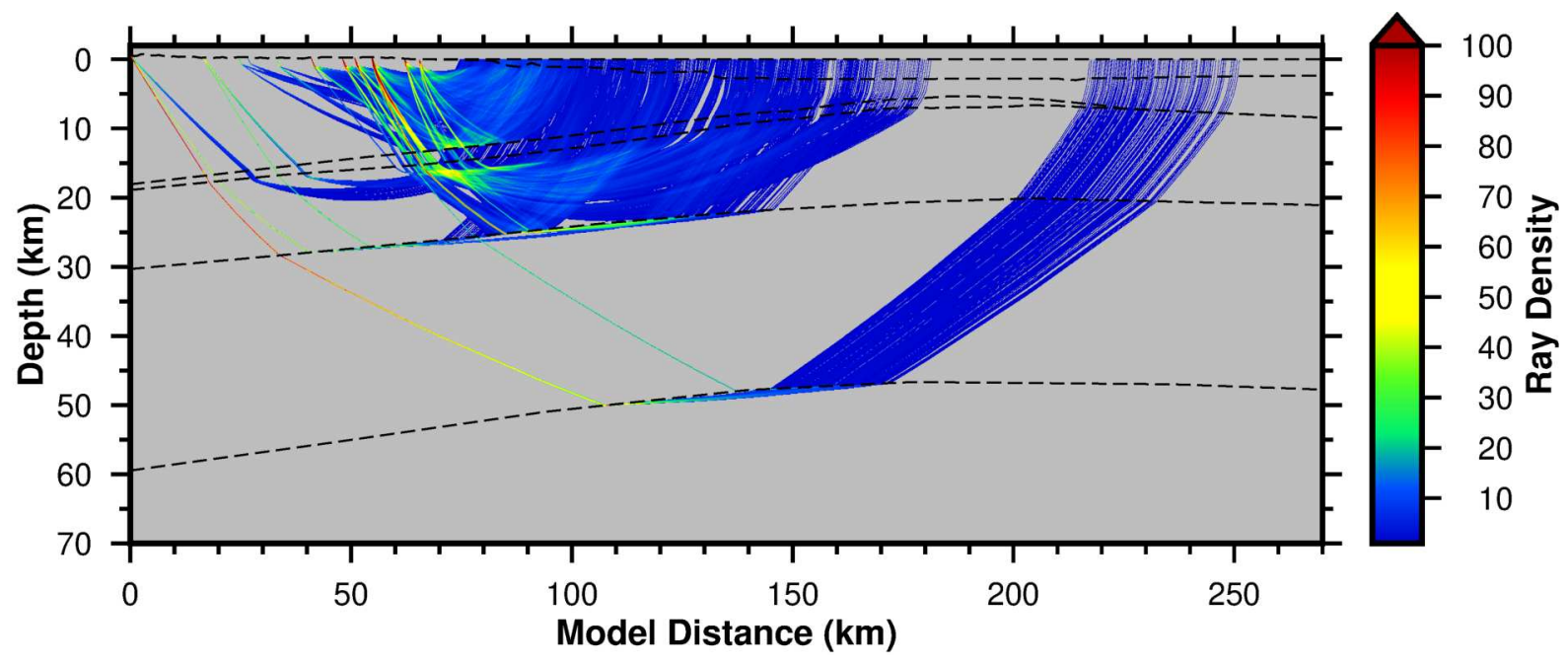

Figure A.28 Ray density plot computed on a $100 \mathrm{~m} \times 100 \mathrm{~m}$ grid along PEGASUS23 transect. Dashed black lines-layer boundaries

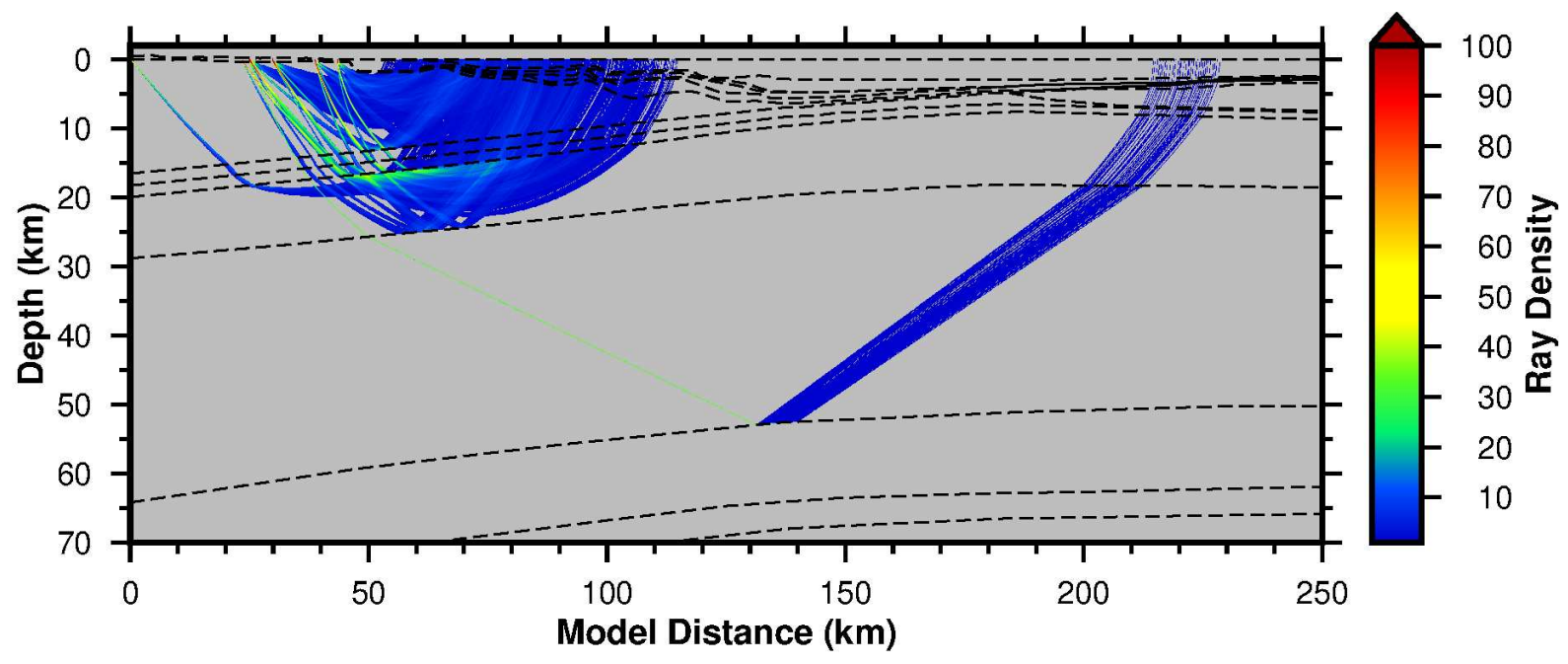

Figure A.29 Ray density plot computed on a $100 \mathrm{~m} \times 100 \mathrm{~m}$ grid along PEGASUS25 transect. Dashed black lines-layer boundaries 


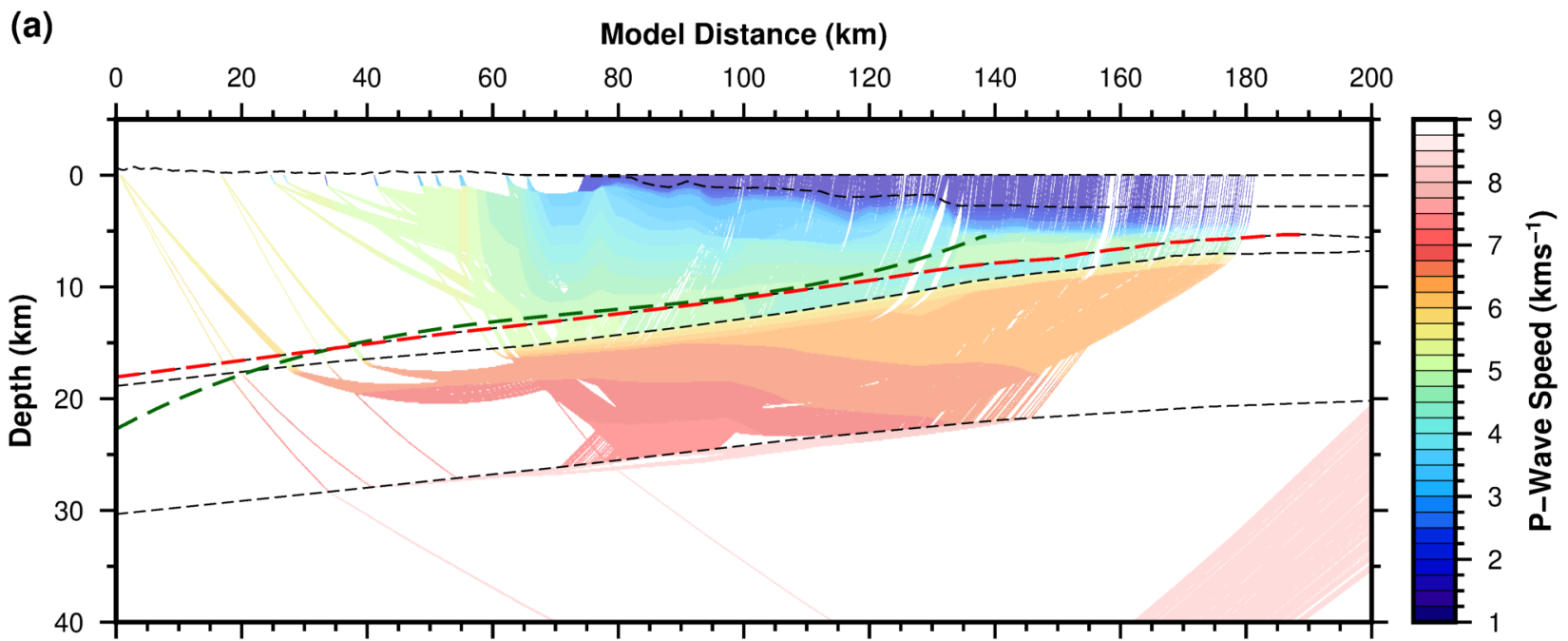

(b)

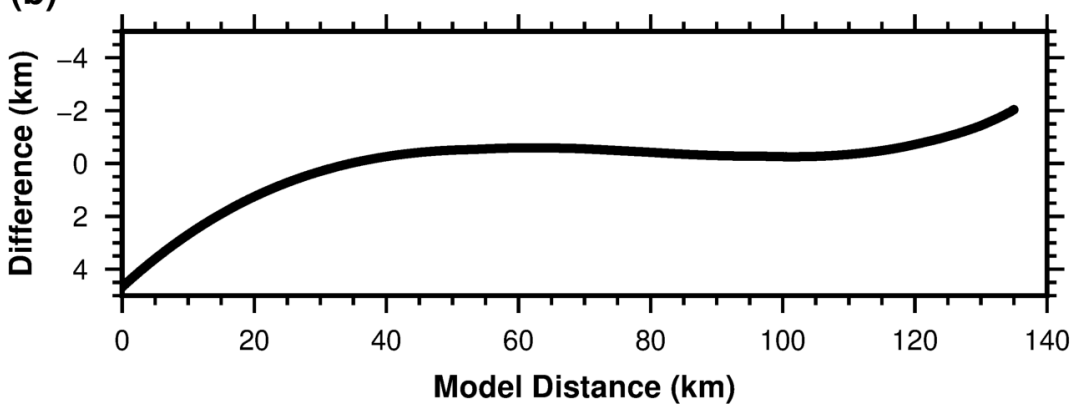

(c)

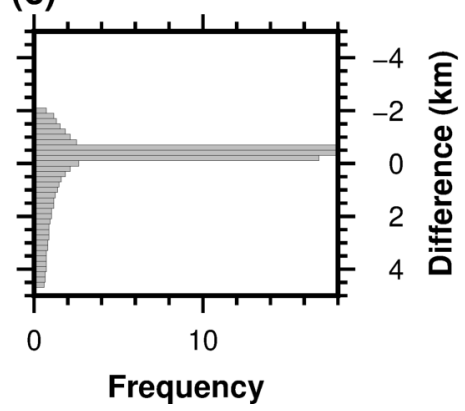

Figure A.30 (a) Comparison of plate interface along PEGASUS23 transect from this study (red dashed line) and from Williams et al. [2013] (green dashed line), superimposed on the velocity model resolved along raypaths. (b) Difference in depth of Williams et al.'s [2013] plate interface and that from this study. Williams et al.'s [2013] plate interface model has been derived by interpolating upper envelopes of relocated earthquakes and the plate interface identified from active source seismic surveys. (c) Histogram of depth differences. 


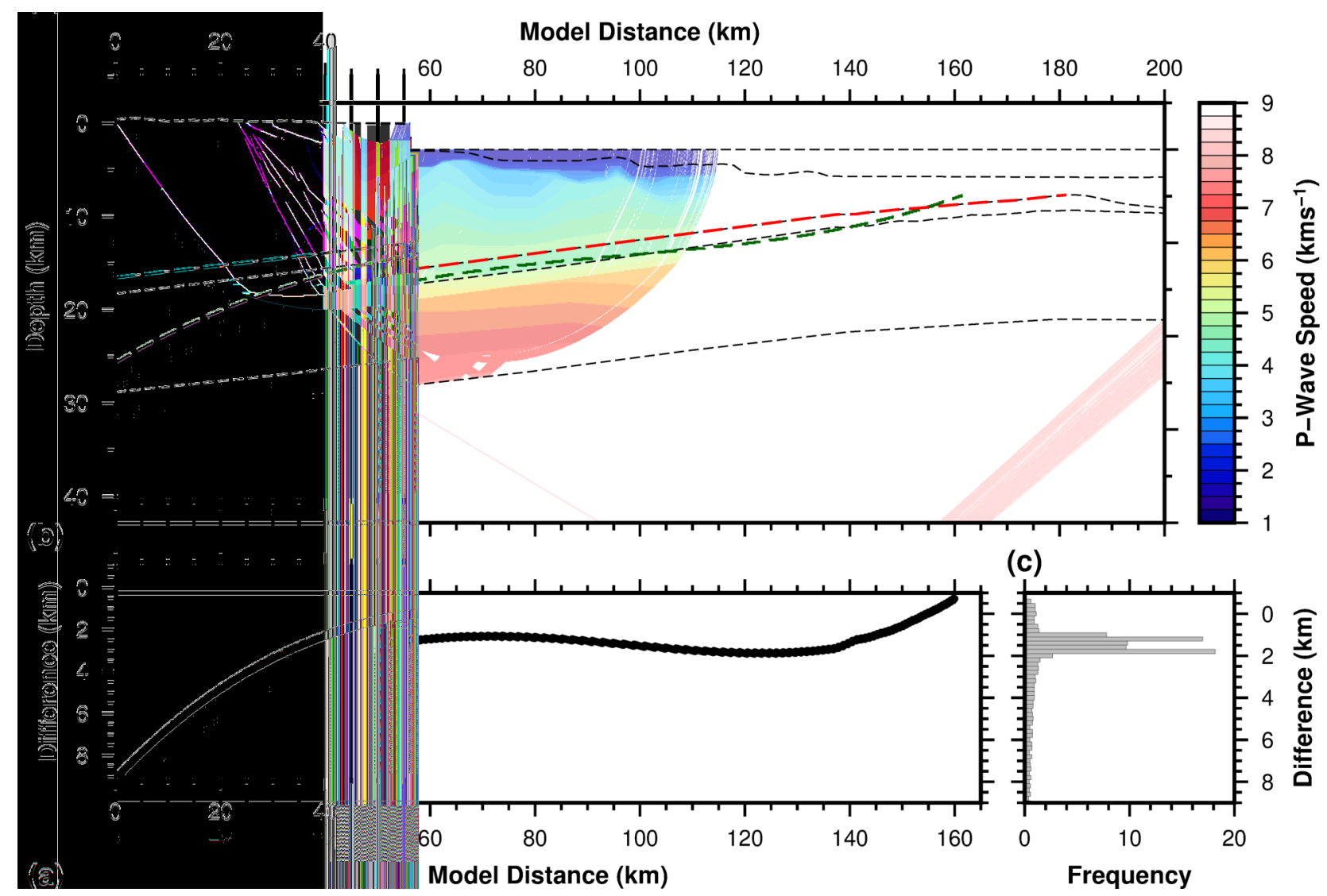

Figure A.31 (a) Comparison of plate interface along PEGASUS25 transect from this study (red dashed line) and from Williams et al. [2013] (green dashed line), superimposed on the velocity model resolved along raypaths. (b) Difference in depth of Williams et al.'s [2013] plate interface and that from this study. Williams et al.'s [2013] plate interface model has been derived by interpolating upper envelopes of relocated earthquakes and the plate interface identified from active source seismic surveys. (c) Histogram of depth differences. 


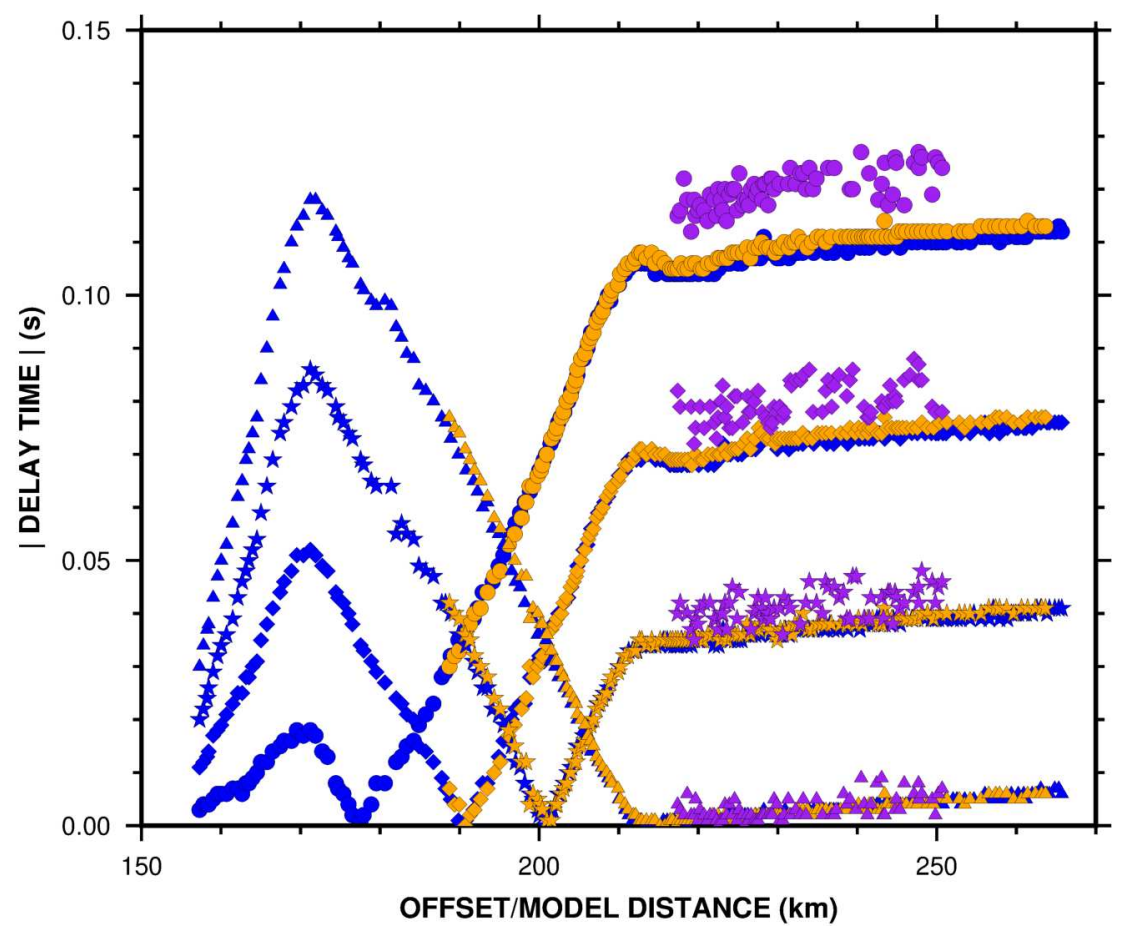

Figure A.32 Delay times for $\mathrm{P}_{\mathrm{n} 2}$ (purple), $\mathrm{R}_{1}$ (orange) and $\mathrm{R}_{2}$ (blue) phases of the common receiver gather at MRZ station with the shots of SAHKE01 line (Figure 4a) when the velocity structure of the Hikurangi Plateau crust at offsets $>150 \mathrm{~km}$ is changed from the final preferred model along PEGASUS23 transect. Base velocity of oceanic crust $6.60 \mathrm{~km} / \mathrm{s}$ (circles), $6.80 \mathrm{~km} / \mathrm{s}$ (diamonds), $7.00 \mathrm{~km} / \mathrm{s}$ (stars) and $7.20 \mathrm{~km} / \mathrm{s}$ (triangles). Note that the thickness of the Hikurangi Plateau crust has not been changed from the model as its thickness is found to be uniform $(\sim 12 \mathrm{~km})$. 
(a)

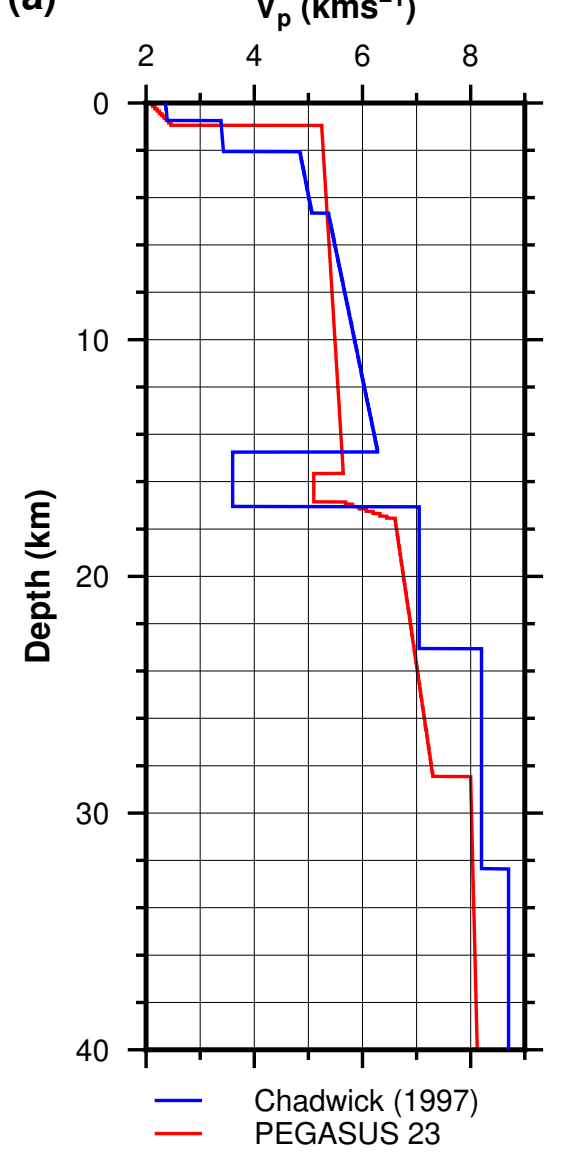

(b)

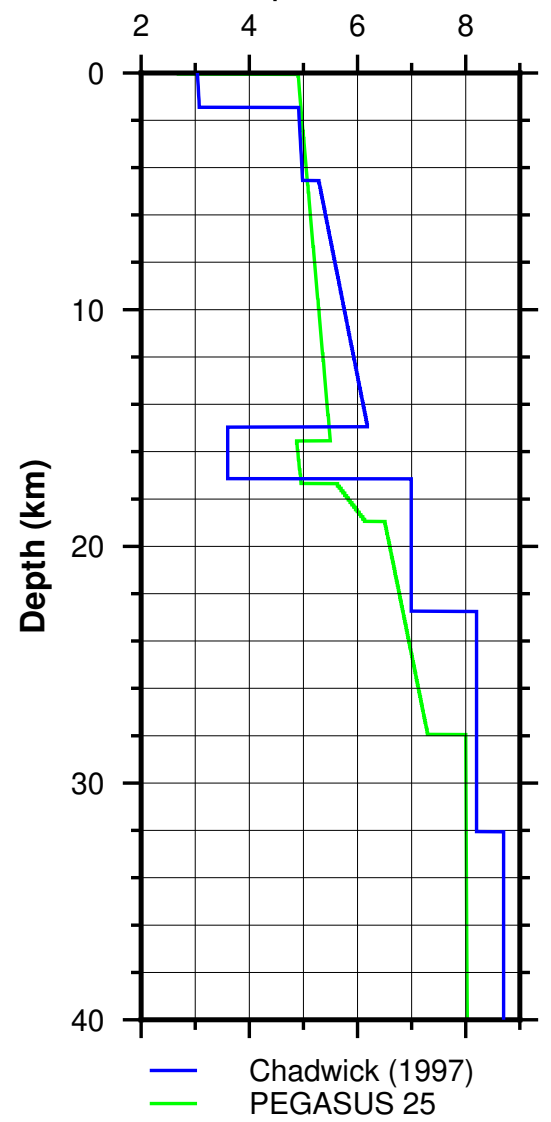

Figure A.33 (a) One-dimensional velocity models of Chadwick's [1997] refraction profile and PEGASUS23 transect at the point of their intersection. (b) One-dimensional velocity models of Chadwick's [1997] refraction profile and PEGASUS25 transect at the point of their intersection.

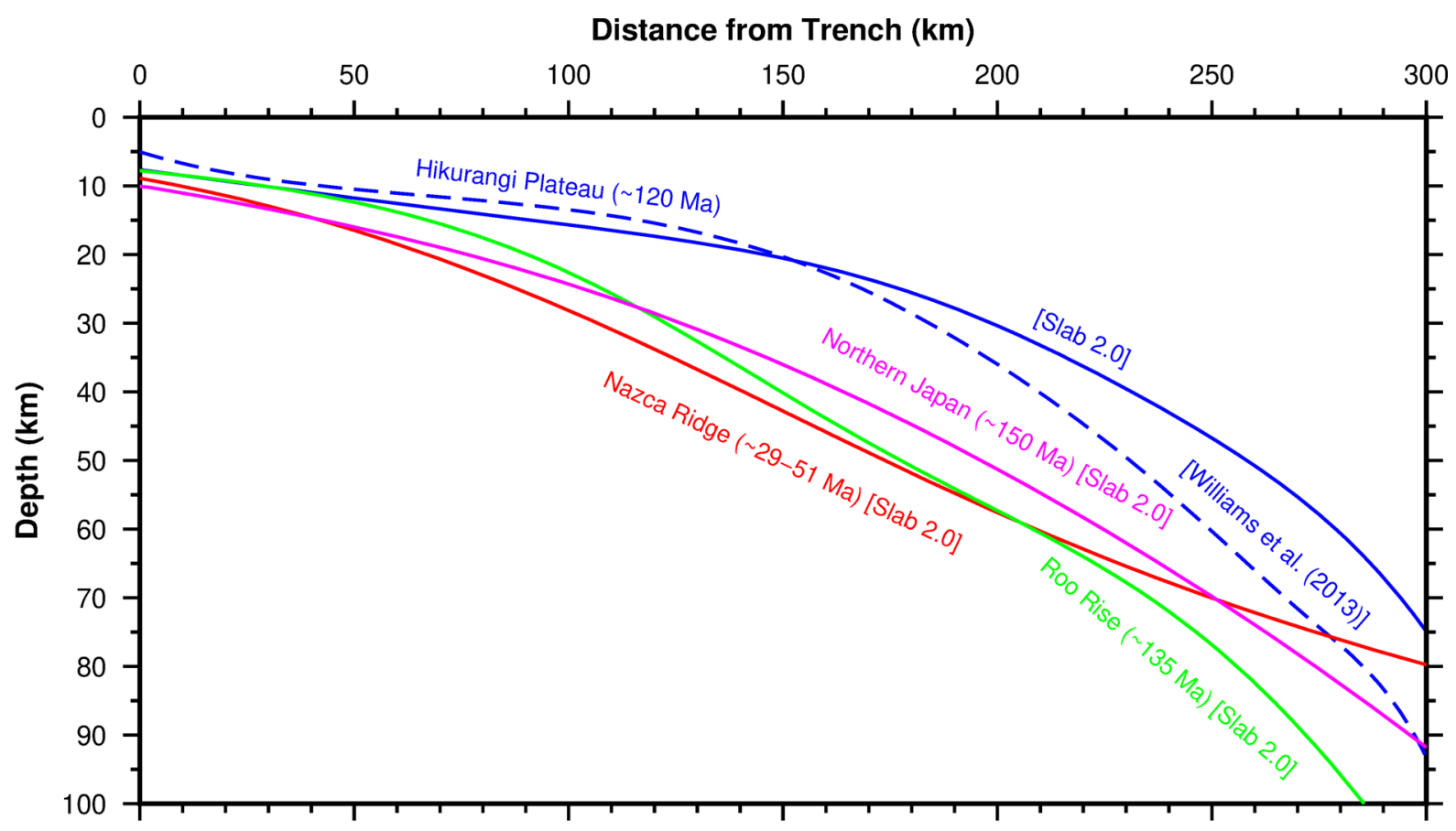

Figure A.34 Comparison of the curvatures of the subducting slabs and their ages. Solid lines show the slab curvatures from the Slab 2.0 model for global subduction zones [Hayes et al., 2018]. Dashed line shows the slab curvature for Hikurangi Plateau from the plate interface model of Williams et al. [2013]. 

Appendix B

Supplementary Material for Chapter 4 


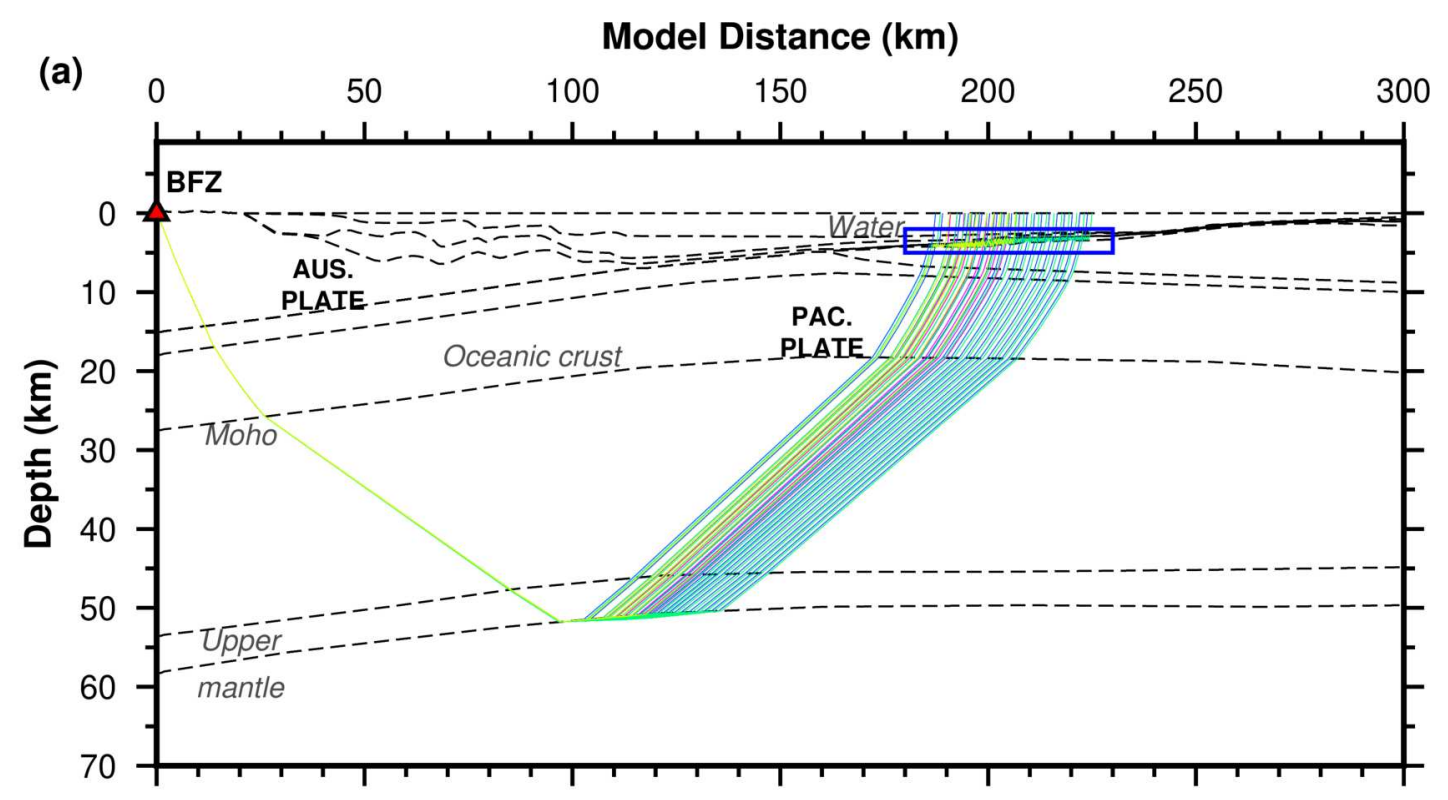

(b)

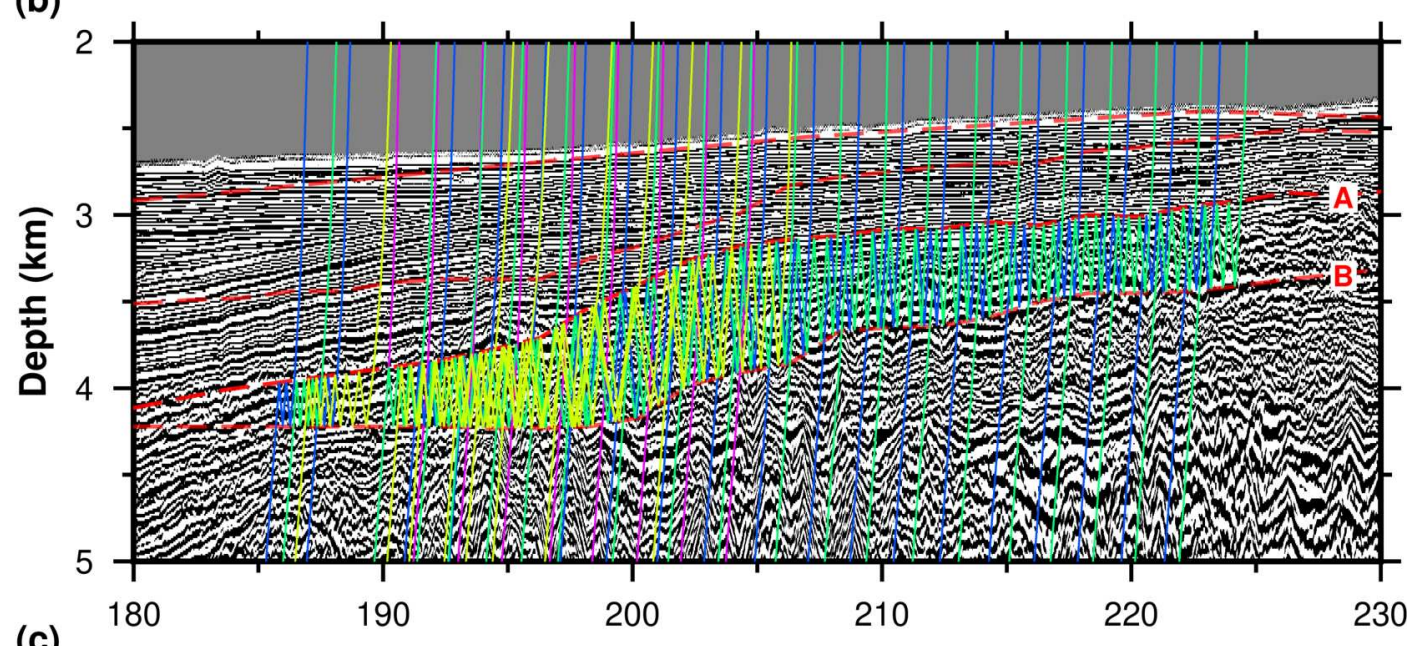

(c)

Model Distance (km)

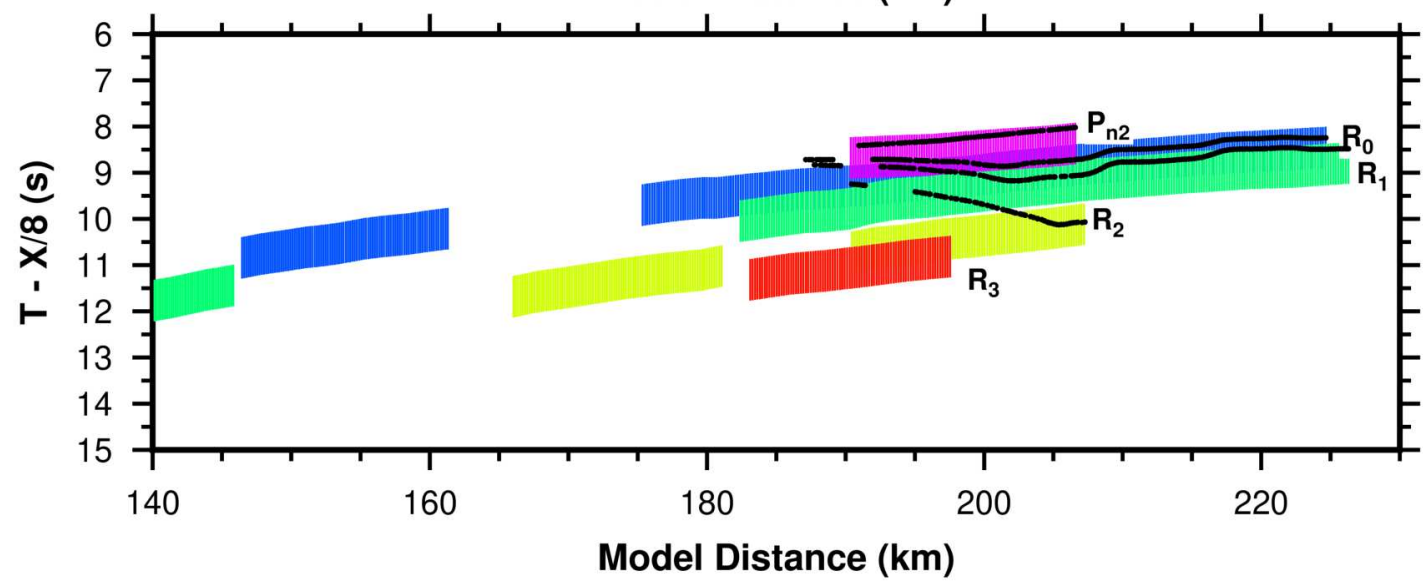

Figure B.1 Simple test to assess whether $R_{0}, R_{1}$ and $R_{2}$ observed in the common receiver gather at BFZ with the airgun shots of SAHKE01 line (Figure 4.2a) could be explained as peg-leg multiples between prominent reflectors within the sedimentary section of $\mathrm{P}_{\mathrm{n} 2}$ upper mantle refracted phase. (a) Structure and raypath diagram. Blue rectangle indicates the extents of (b). (b) Zoomed section of the depth converted multichannel seismic reflection image along SAHKE01 showing the two prominent reflectors A and B in the sedimentary section. (c) Observed (colour-coded as in (a) and (b)) and calculated traveltimes (black). 


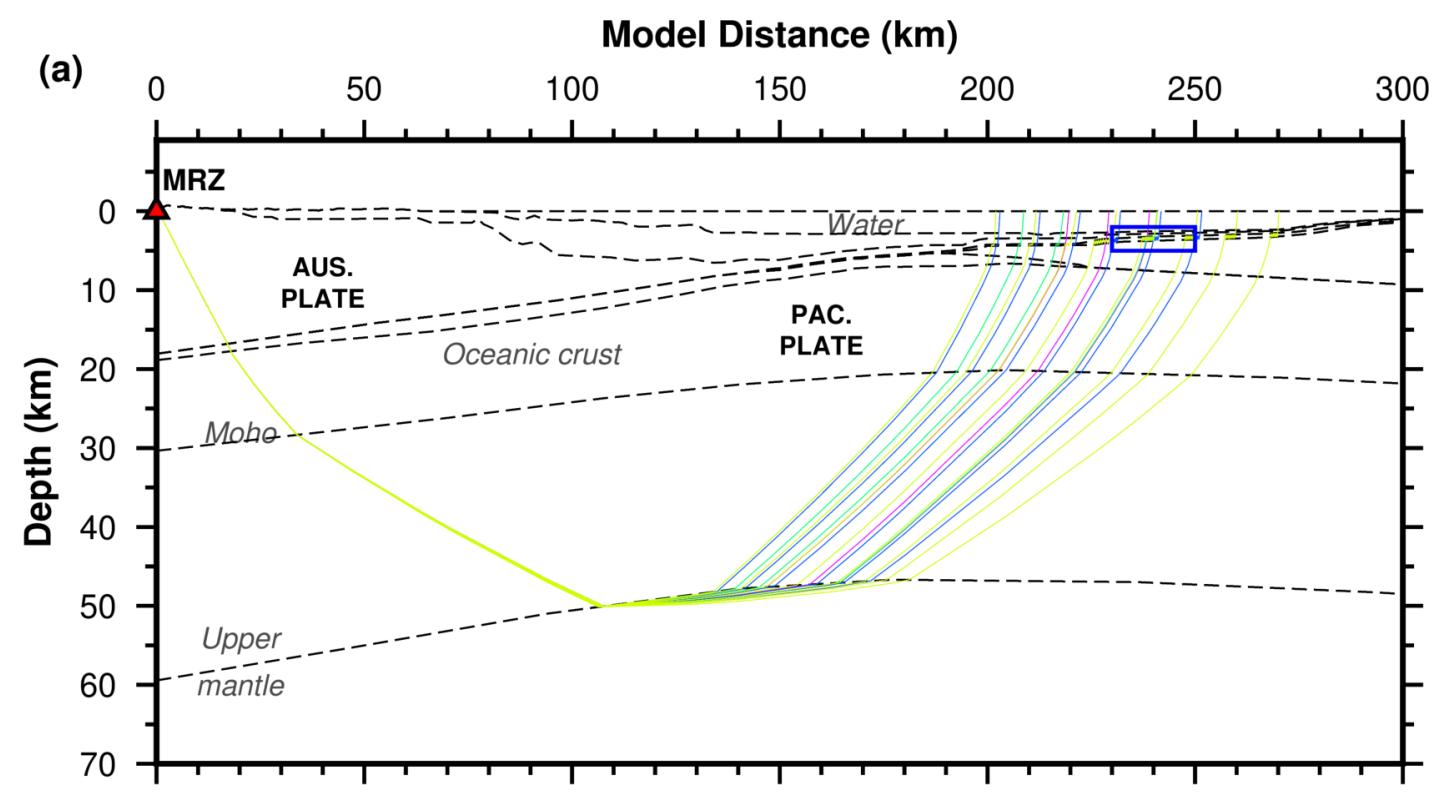

(b)

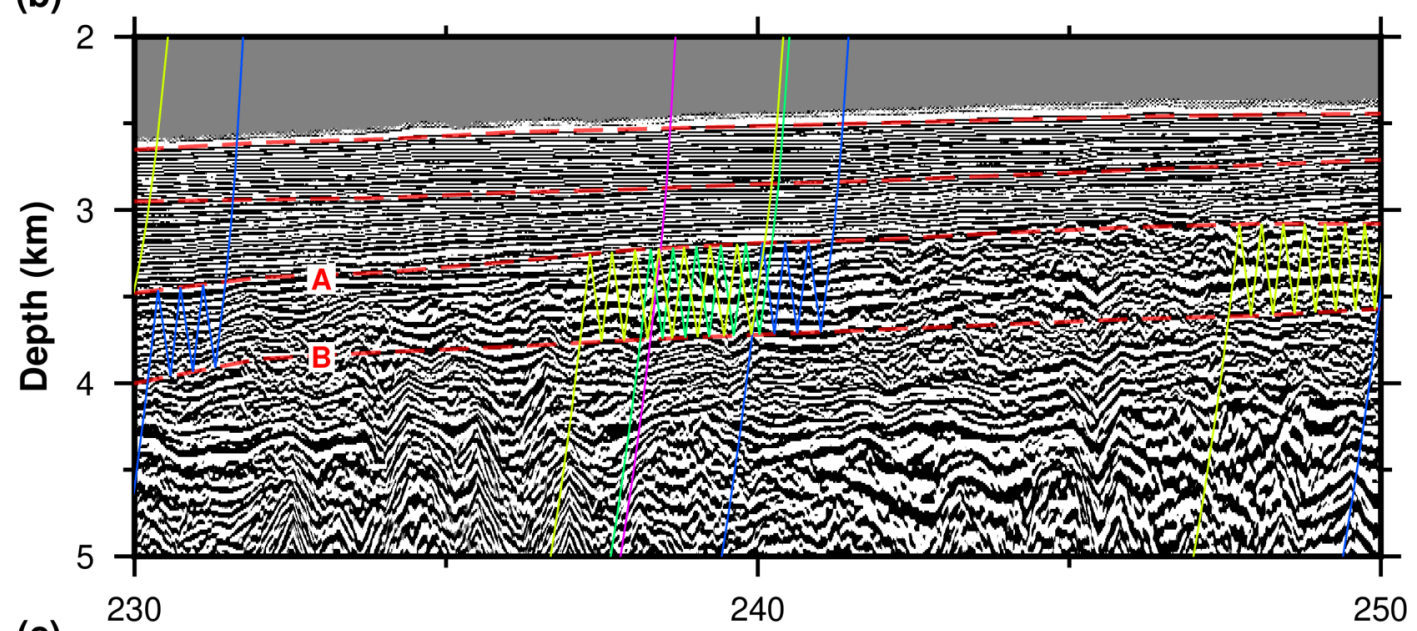

(c)

Model Distance (km)

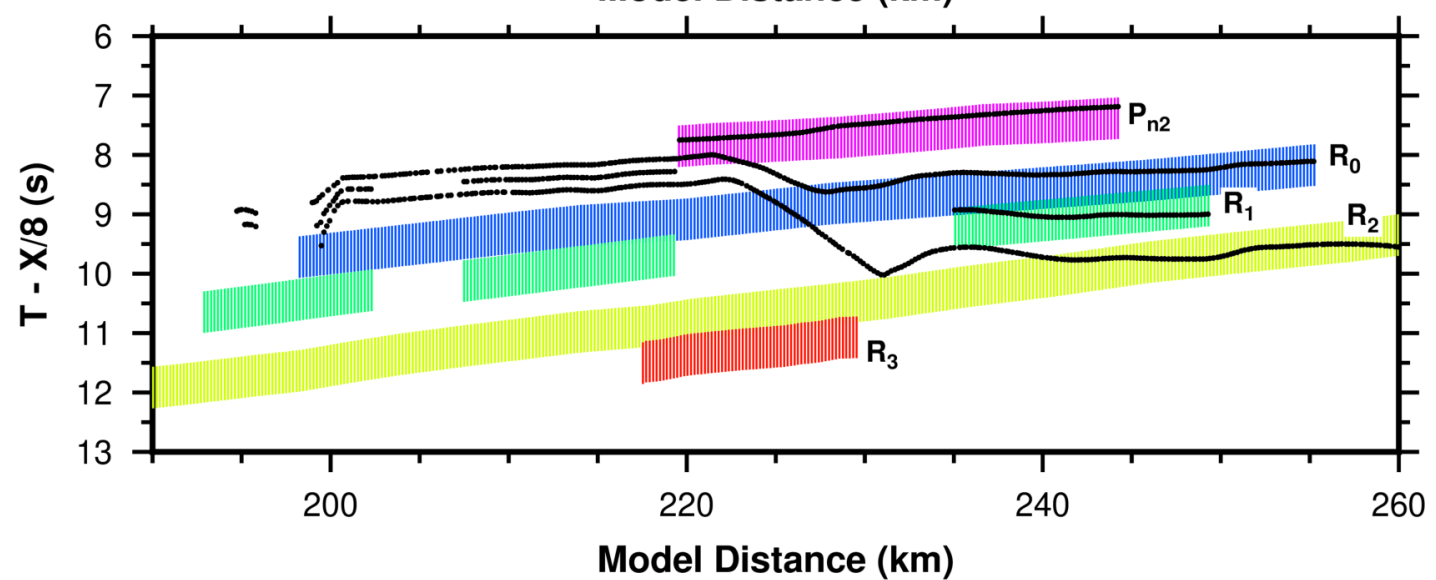

Figure B.2 Simple test to assess whether $R_{0}, R_{1}$ and $R_{2}$ observed in the common receiver gather at MRZ with the airgun shots of SAHKE01 line (Figure 4.2b) could be explained as peg-leg multiples between prominent reflectors within the sedimentary section of $\mathrm{P}_{\mathrm{n} 2}$ upper mantle refracted phase. (a) Structure and raypath diagram. Blue rectangle indicates the extents of (b). (b) Zoomed section of the depth converted multichannel seismic reflection image along SAHKE01 showing the two prominent reflectors A and B in the sedimentary section. (c) Observed (colour-coded as in (a) and (b)) and calculated traveltimes (black). 


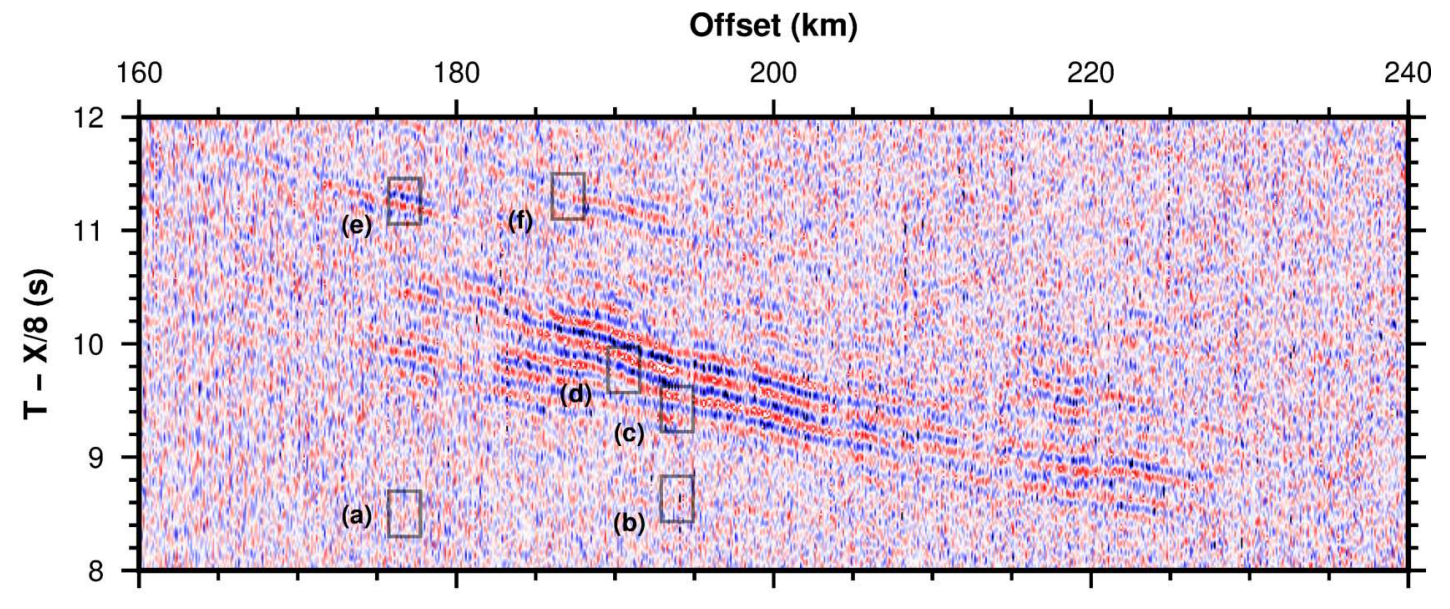

(a) Noise

(b) $P_{n 2}$

(c) $\mathrm{R}_{\mathbf{0}}$

(d) $R_{1}$

(e) $\mathbf{R}_{\mathbf{2}}$

(f) $\mathbf{R}_{3}$

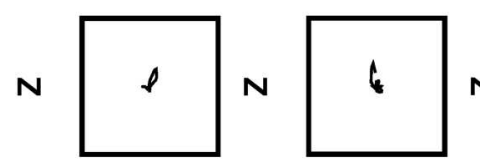

$\mathbf{R}$

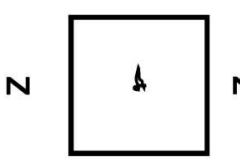

T

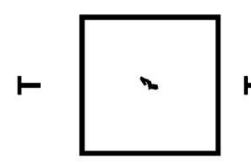

$\mathbf{R}$
$\mathbf{R}$

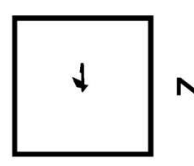

T

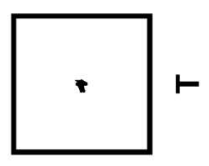

$\mathbf{R}$

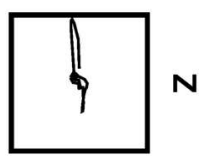

$\mathbf{R}$

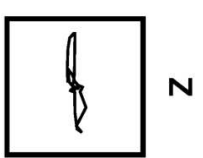

$\mathbf{R}$

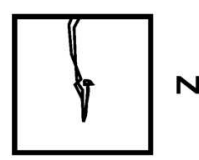

$\mathbf{R}$

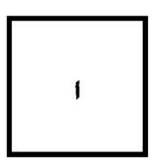

$\mathbf{R}$

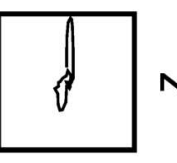

T

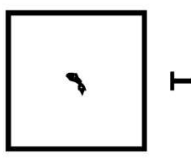

$\mathbf{R}$

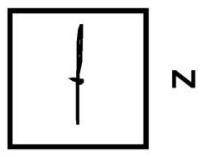

$\mathbf{T}$

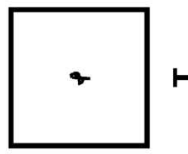

$\mathbf{R}$

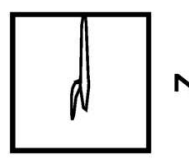

T

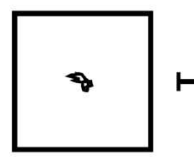

$\mathbf{R}$

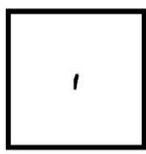

$\mathbf{T}$

Figure B.3 Particle motion plots to test if (a) $P_{n 2}$, (b) $R_{0}$, (c) $R_{1}$, (d) $R_{2}$ and (e) $R_{3}$ are incoming shear waves into the onshore seismograph (BFZ) with airgun shots of SAHKE01 line. Particle motion corresponds to the trace at the center of each rectangle within a time window of $400 \mathrm{msec}$ (shown by the height of each rectangle).

Table B.1 Raytracing statistics for the wide-angle reflections observed in the common receiver gathers shown in Figure 4.2 in the main text.

\begin{tabular}{ccccccccccccc}
\hline \multirow{2}{*}{ Phase } & \multicolumn{3}{c}{ MRZ/PEGASUS23 } & \multicolumn{4}{c}{ MRZ/SAHKE01 } & \multicolumn{4}{c}{ BFZ/SAHKE01 } \\
& Av. Unc. & $\mathbf{N}$ & $\mathbf{T}_{\text {RMS }}$ & $\chi^{2}$ & Av. Unc. & $\mathbf{N}$ & $\mathbf{T}_{\text {RMS }}$ & $\chi^{2}$ & Av. Unc. & $\mathbf{N}$ & $\mathbf{T}_{\text {RMs }}$ & $\chi^{2}$ \\
\hline $\mathbf{R}_{\mathbf{0}}$ & na & na & na & na & 0.200 & 590 & 0.084 & 0.175 & 0.200 & 741 & 0.202 & 1.021 \\
$\mathbf{R}_{\mathbf{1}}$ & 0.200 & 265 & 0.191 & 0.914 & 0.200 & 372 & 0.219 & 1.203 & 0.200 & 654 & 0.132 & 0.435 \\
$\mathbf{R}_{\mathbf{2}}$ & 0.200 & 439 & 0.039 & 0.038 & 0.200 & 1112 & 0.141 & 0.496 & 0.200 & 368 & 0.115 & 0.335 \\
$\mathbf{R}_{\mathbf{3}}$ & na & na & na & na & 0.200 & 126 & 0.130 & 0.433 & 0.200 & 166 & 0.052 & 0.067 \\
\hline
\end{tabular}

Av. Unc. $=$ Average uncertainty in travel-time picks

$\mathrm{N}=$ Number of travel-time picks

$\mathrm{T}_{\mathrm{RMS}}=$ Root mean square travel-time misfit 


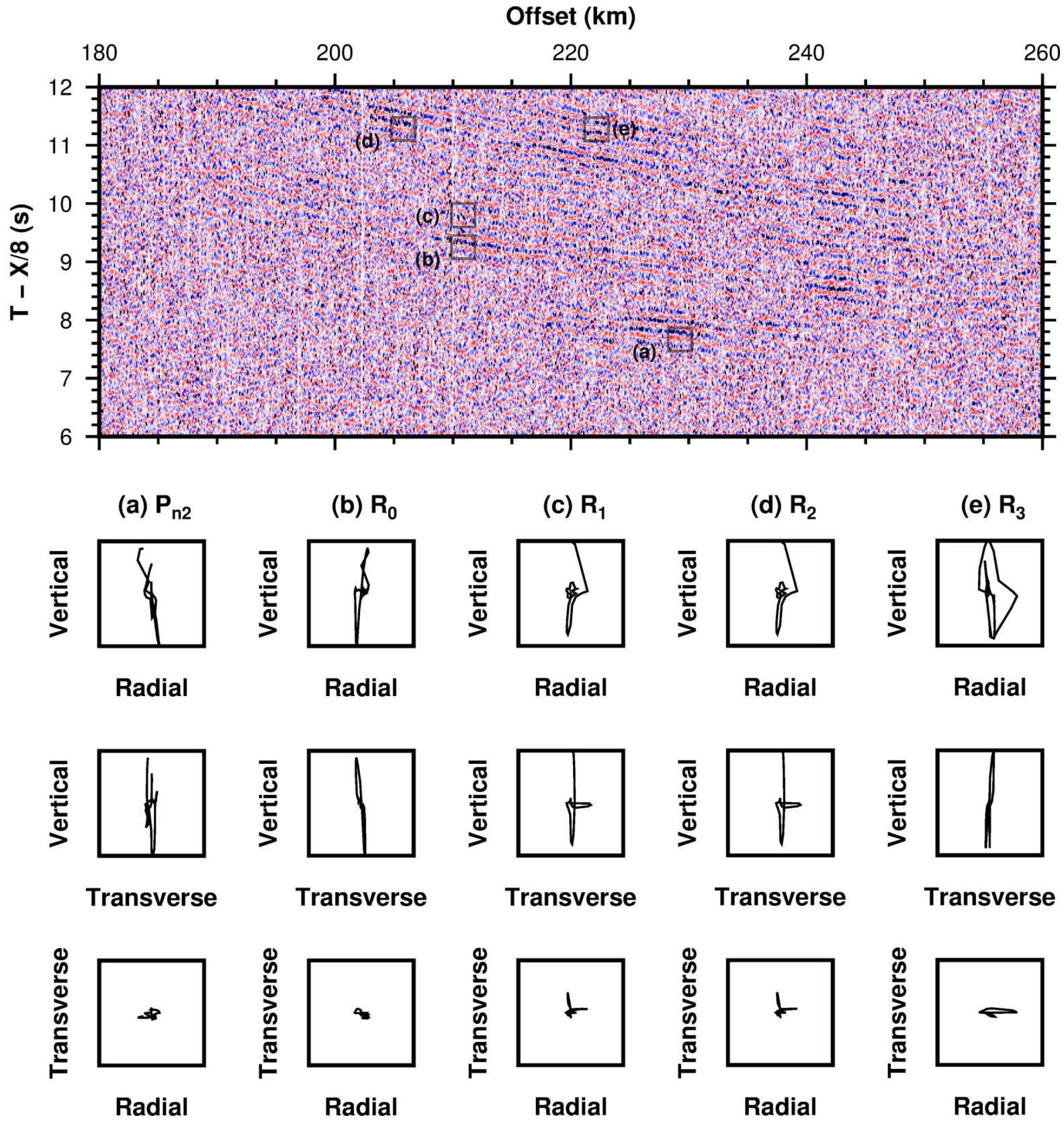

Figure B.4 Particle motion plots to test if (a) $P_{n 2}$, (b) $R_{0}$, (c) $R_{1}$, (d) $R_{2}$ and (e) $R_{3}$ are incoming shear waves into the onshore seismograph (MRZ) with airgun shots of SAHKE01 line. Particle motion corresponds to the trace at the center of each rectangle within a time window of $400 \mathrm{msec}$ (shown by the height of each rectangle).

Stiffness tensor for anisotropic layer $=\left[\begin{array}{ccccccc}291.44 & 75.78 & 81.67 & -1.42 & -14.59 & 4.67 \\ 75.78 & 242.58 & 80.26 & -0.89 & 0.35 & 3.12 \\ 81.67 & 80.26 & 234.35 & -0.89 & -13.03 & 0.95 \\ -1.42 & -0.89 & -0.89 & 75.99 & 2.64 & -4.94 \\ -14.59 & 0.35 & -13.03 & 2.64 & 90.01 & -2.4 \\ 4.67 & 3.12 & 0.95 & -4.94 & -2.4 & 82.09\end{array}\right]$




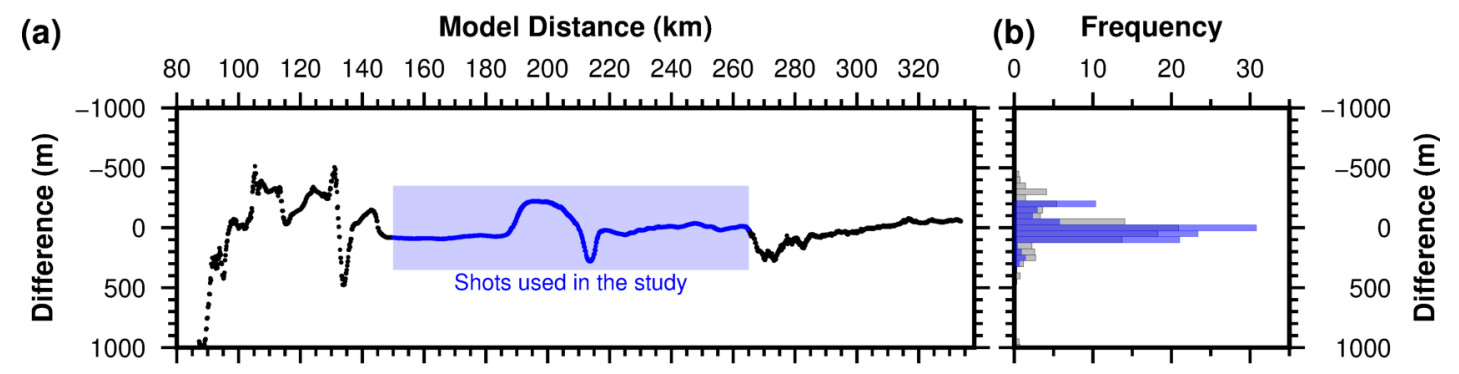

Figure B.5 (a) Difference in water depth between the original location of the airgun shots along the SAHKE01 line and their projected locations on to the PEGASUS23 line. Note the hump near $200 \mathrm{~km}$ model distance is due to the Hikurangi channel (b) Histogram of the depth differences in (a).

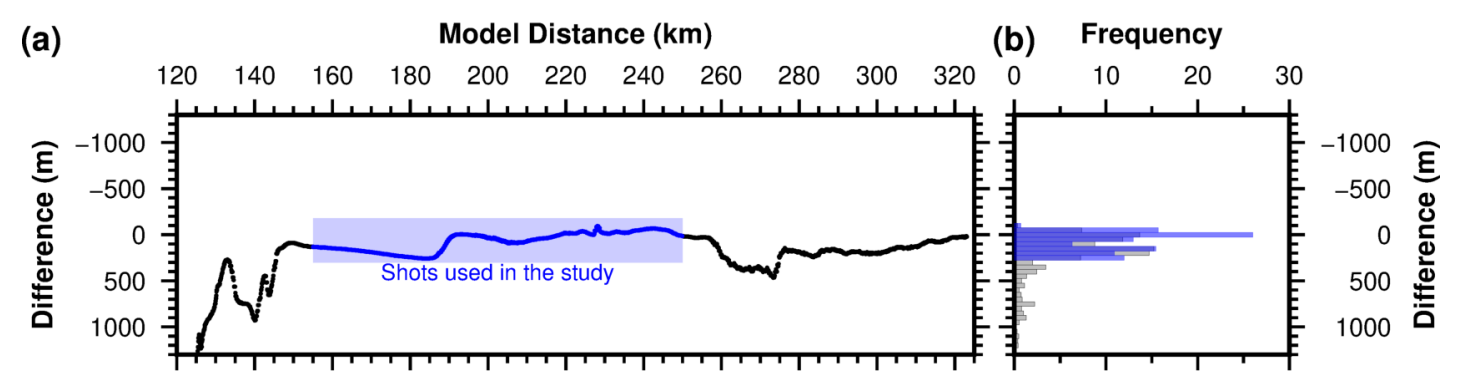

Figure B.6 (a) Difference in water depth between the original location of the airgun shots along the SAHKE01 line and their projected locations on to the PEGASUS25 line. (b) Histogram of the depth differences in $(\mathrm{a})$.

Table B.2 Density (in $\mathrm{kgm}^{-3}$ ) and velocity (in $\mathrm{kms}^{-1}$ ) parameters used for synthetic waveform modelling in SPECFEM2D.

\begin{tabular}{lccc}
\hline Layer & Density & $\mathbf{V}_{\mathbf{P}}$ & $\mathbf{V}_{\mathbf{P}} / \mathbf{V}_{\mathbf{S}}$ \\
\hline Water & 1000 & 1500 & $\infty$ \\
Upper Crust & 2400 & 4500 & 1.9 \\
Oceanic Crust & 2700 & 6700 & 1.8 \\
Upper Lithospheric Mantle & 3300 & 8000 & 1.8 \\
Lower Lithospheric Mantle & 3300 & 8700 & 1.8 \\
Anisotropic Layer & 3350 & Stiffness & Tensor* \\
Low-velocity Channel (Upper) & 3100 & 7600 & 2.5 \\
Low-velocity Channel (Lower) & 3100 & $7400 / 7800$ & 1.85 \\
Asthenosphere & 3300 & 8100 & 1.8 \\
\hline
\end{tabular}




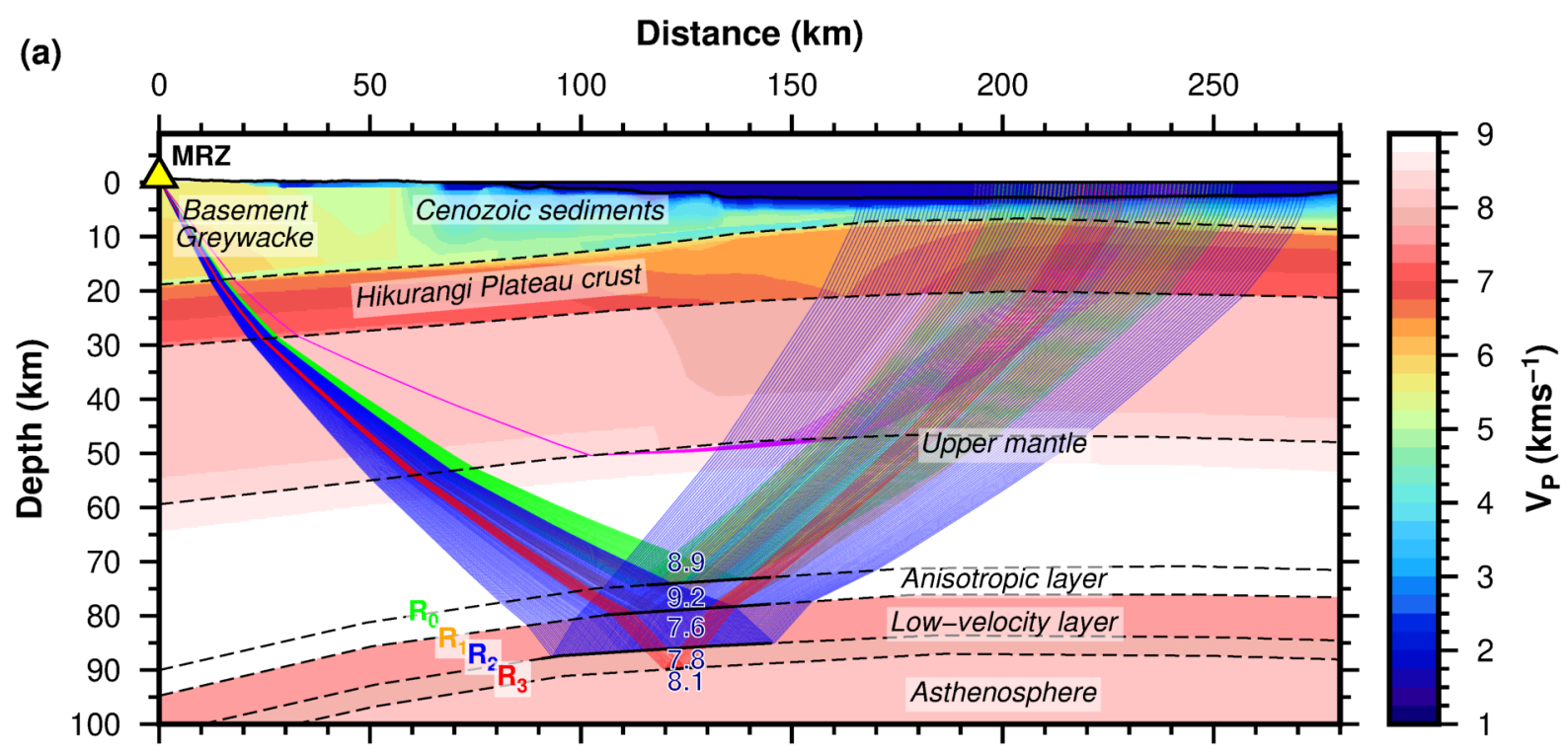

(b)

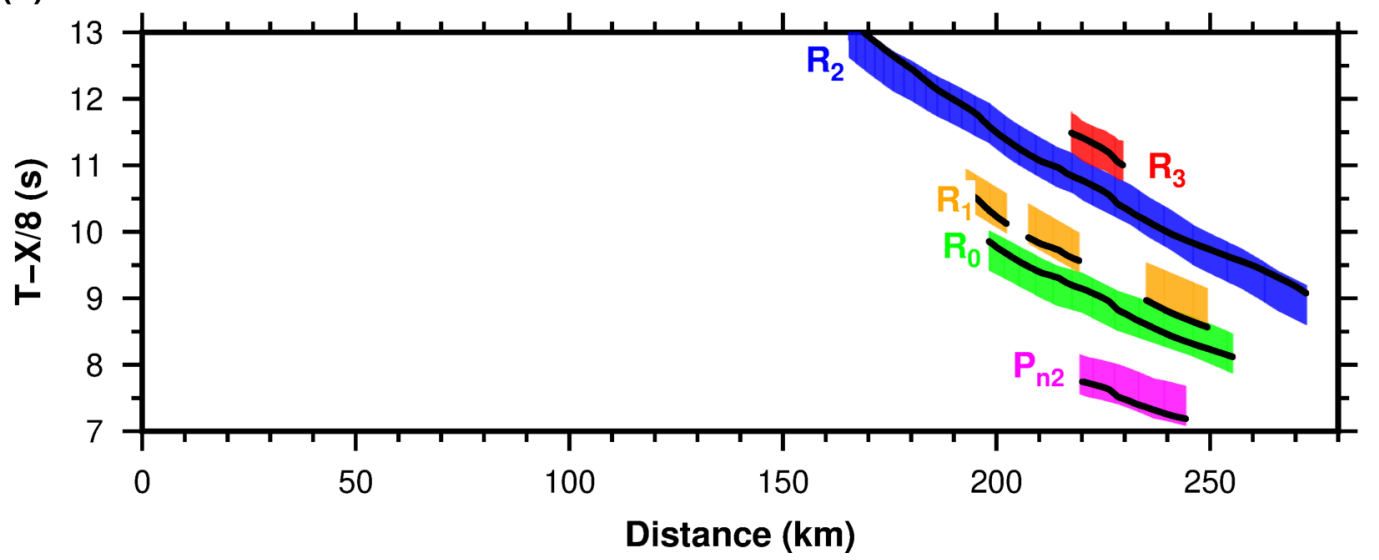

Figure B.7 (a) Velocity model and traced rays for the upper mantle refraction and wide-angle reflections in the common receiver gather at MRZ station with the airgun shots of PEGASUS23 line (Figure 4.2(c)). $\mathrm{P}_{\text {n2-purple, }} \mathrm{R}_{0}$-green, $\mathrm{R}_{1}$-yellow, $\mathrm{R}_{2}$-blue and $\mathrm{R}_{3}$-red. (b) Observed travel-times colour-coded as in (a) and calculated travel-times (black dots). Note that the height of an observed travel-time pick is twice the pick uncertainty. 


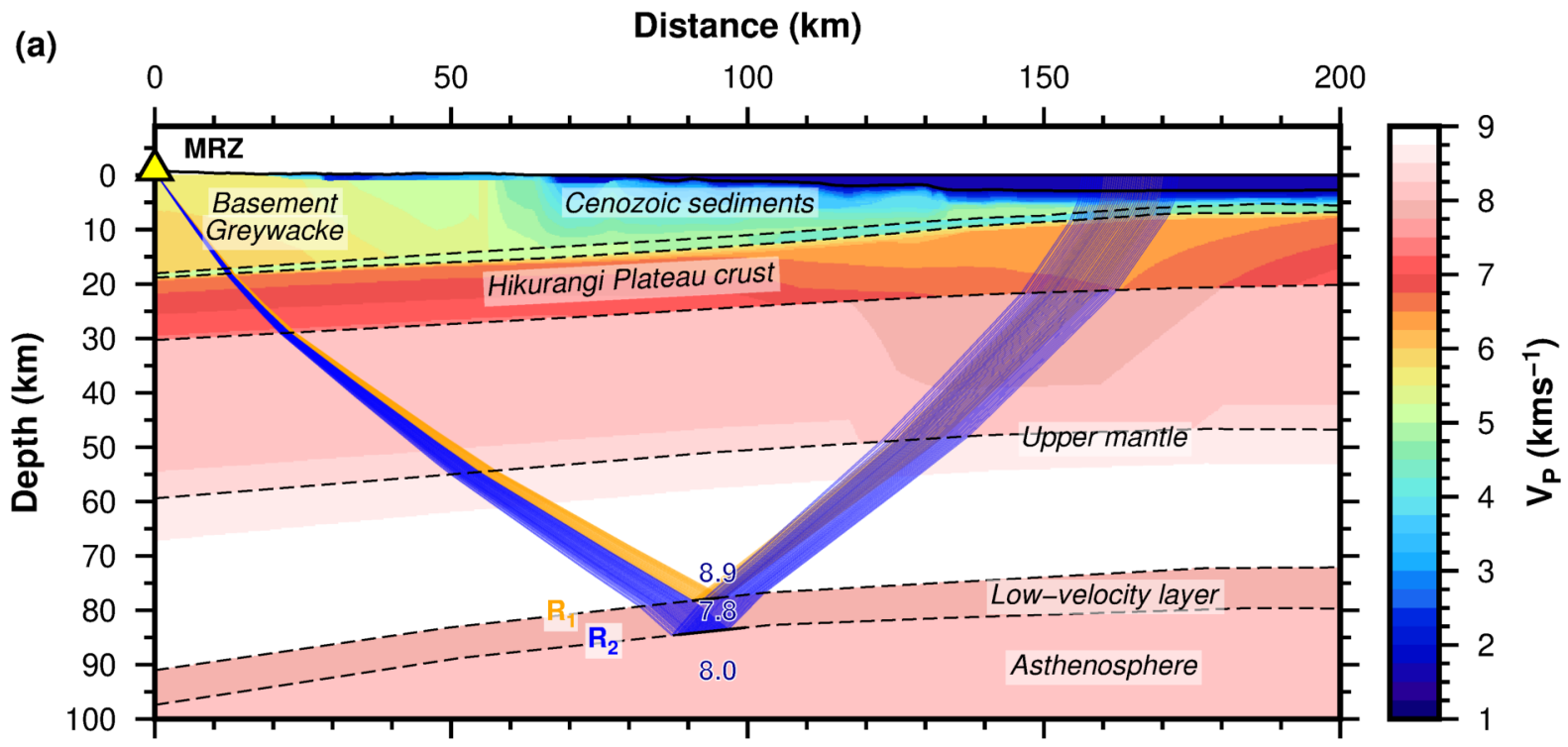

(b)

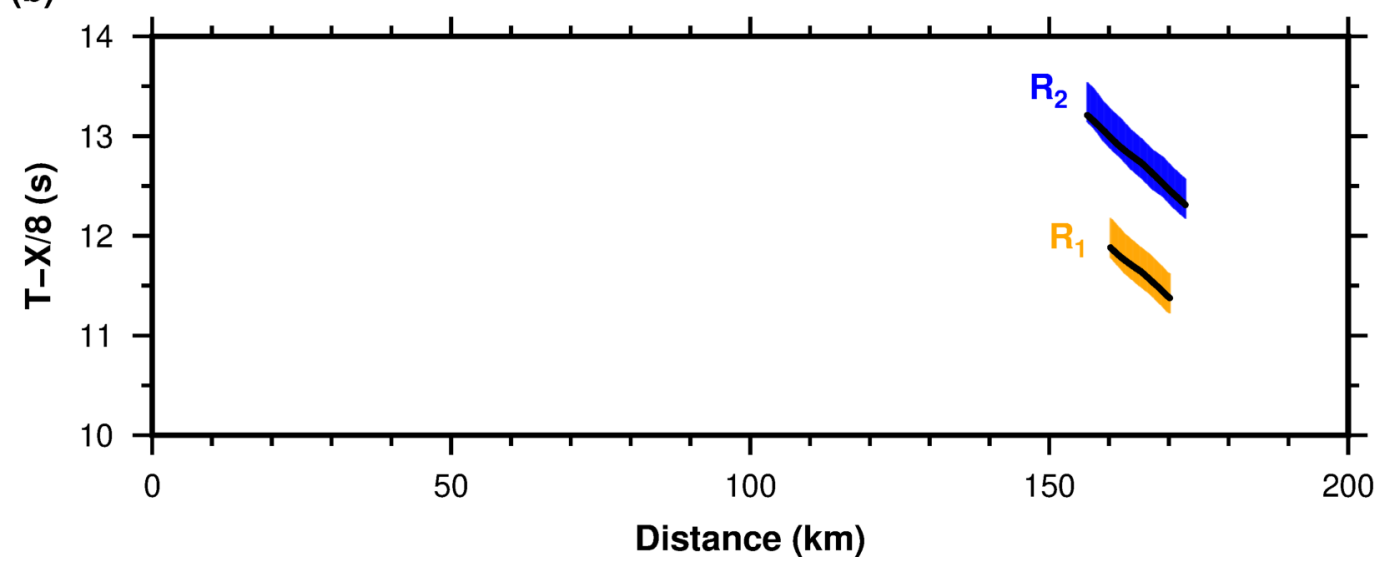

Figure B.8 (a) Velocity model and traced rays for the upper mantle refraction and wide-angle reflections in the common receiver gather at MRZ station with the airgun shots of PEGASUS23 line (Figure 4.2(c)). $\mathrm{R}_{1}$-yellow and $\mathrm{R}_{2}$-blue. (b) Observed travel-times colour-coded as in (a) and calculated travel-times (black dots). Note that the height of an observed travel-time pick is twice the pick uncertainty.

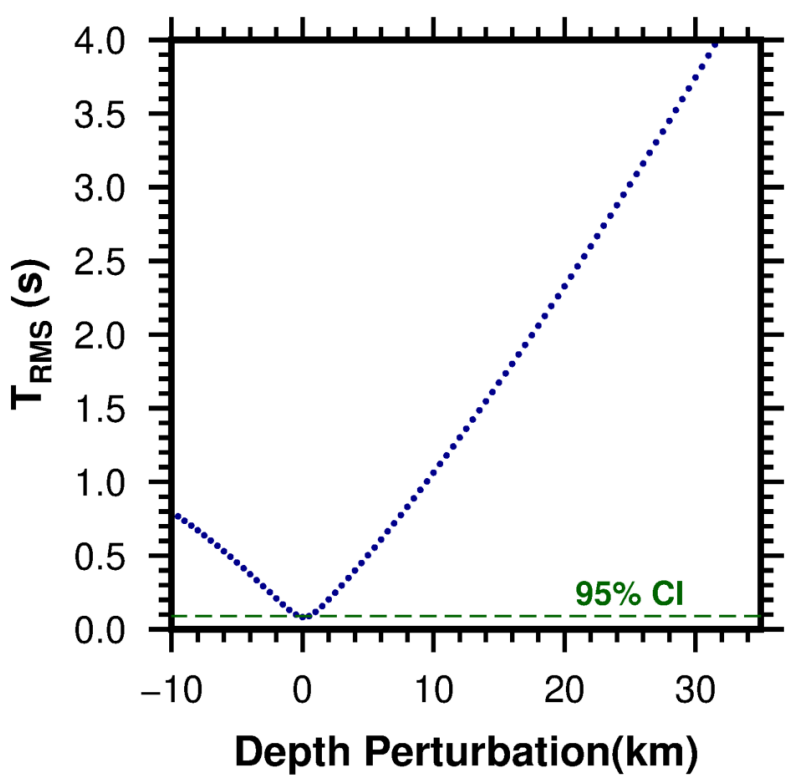

Figure B.9 Uncertainty estimate for the depth to $\mathrm{R}_{0}$ reflection observed in the common receiver gather at BFZ station from the airgun shots of SAHKE01 line. 


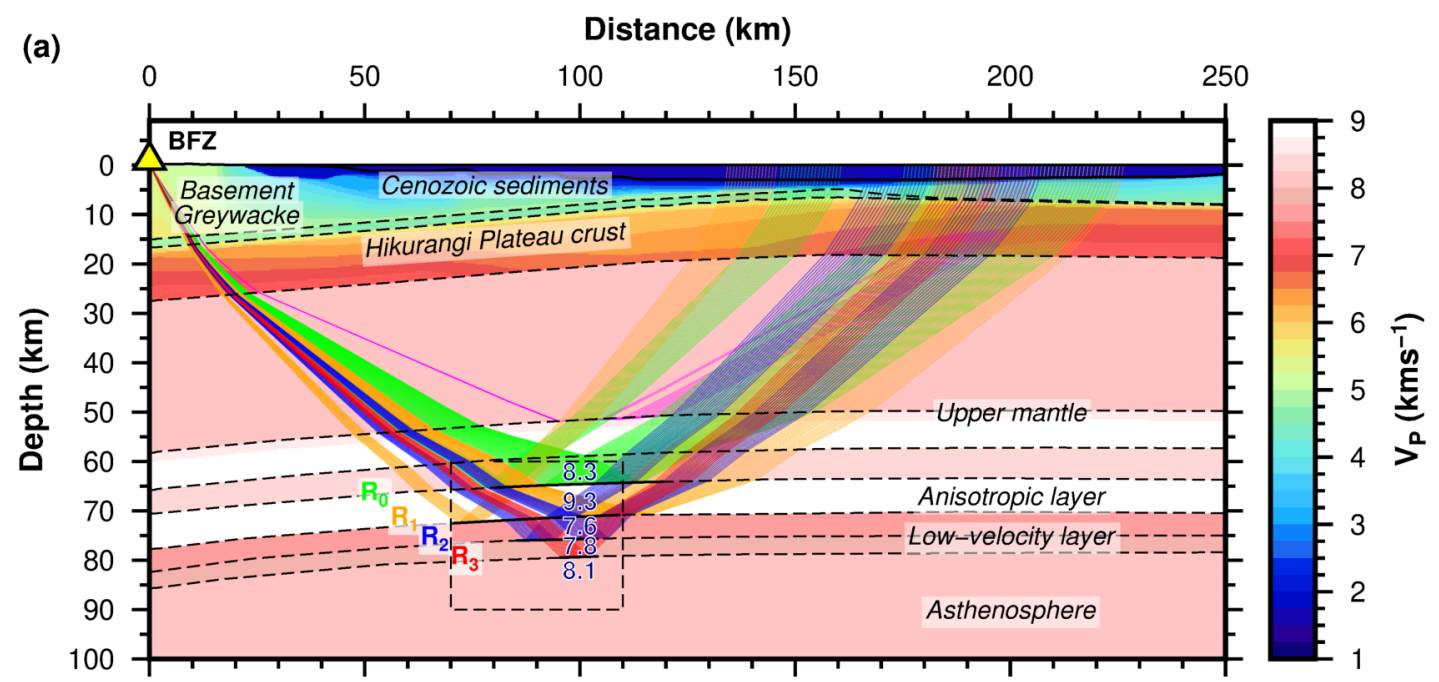

(b)

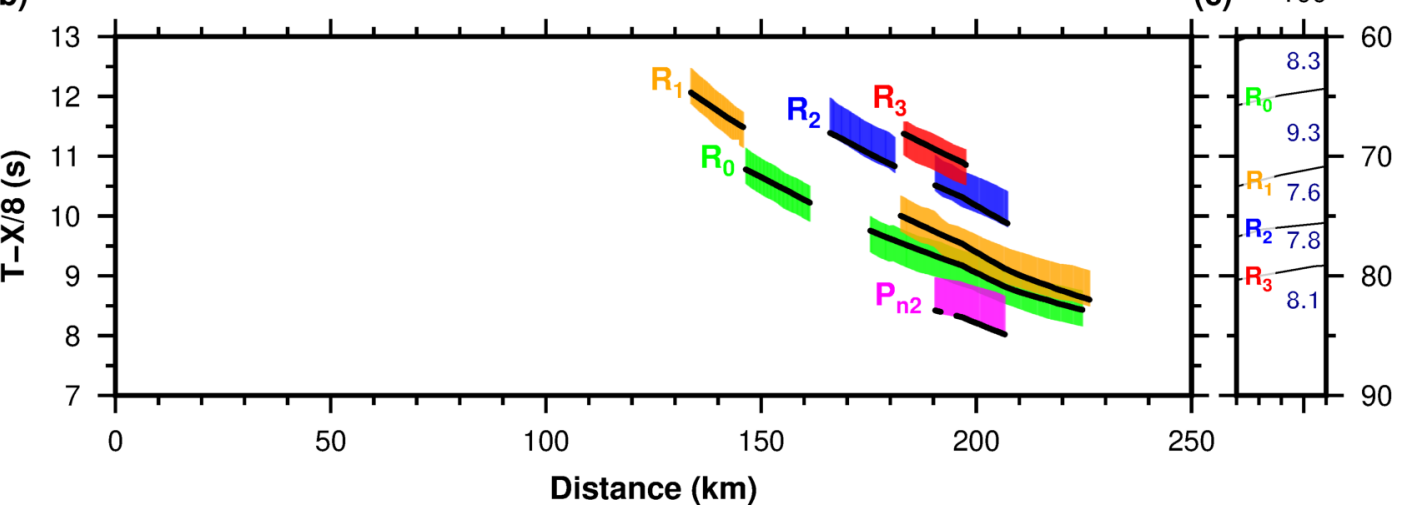

Figure B.10 (a) Raypath diagram for the traced rays for the observed wide-angle reflections and refractions in Figure 4.2a. (b) Travel-time picks of the phases observed in Figure 4.2a colour-coded as in (a). Height of an observed travel-time pick is twice the pick uncertainty. Calculated travel-times are in black. Note that the travel-time axis is reduced with a velocity of $8.0 \mathrm{~km} / \mathrm{s}$. (c) Zoomed-up section of the region shown by the black, dashed rectangle in (a). 

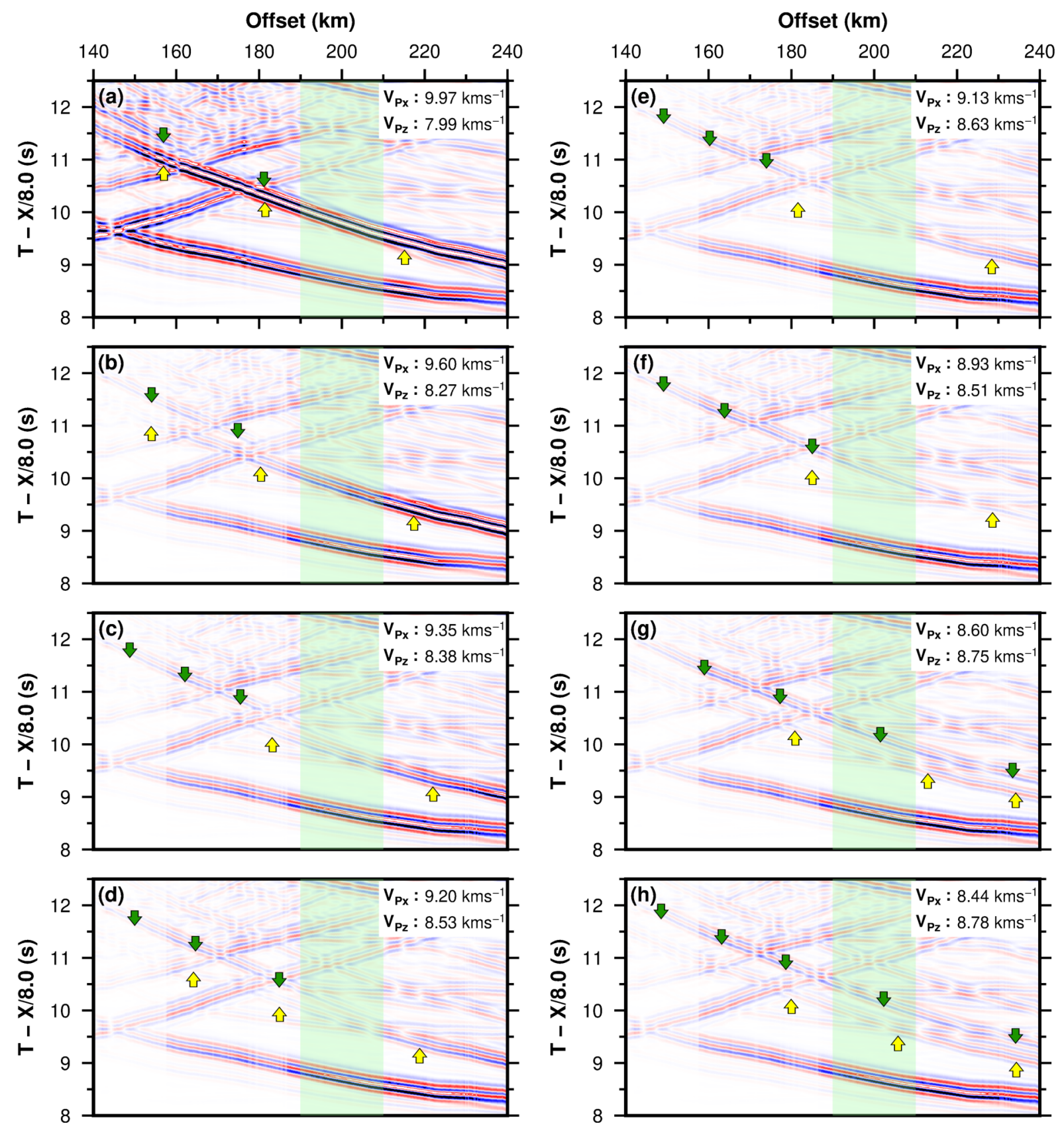

Figure B.11 (a)-(h) Synthetic common receiver gathers for different elastic tensors defining the velocities in the anisotropic layer. Yellow and green arrows indicate $R_{0}$ and $R_{1}$ wide-angle reflections. Light-green shaded region indicates the offset range where $R_{0}$ is observed to merge with $R_{1}$ in the actual common receiver gather in Figure 4.2a. 

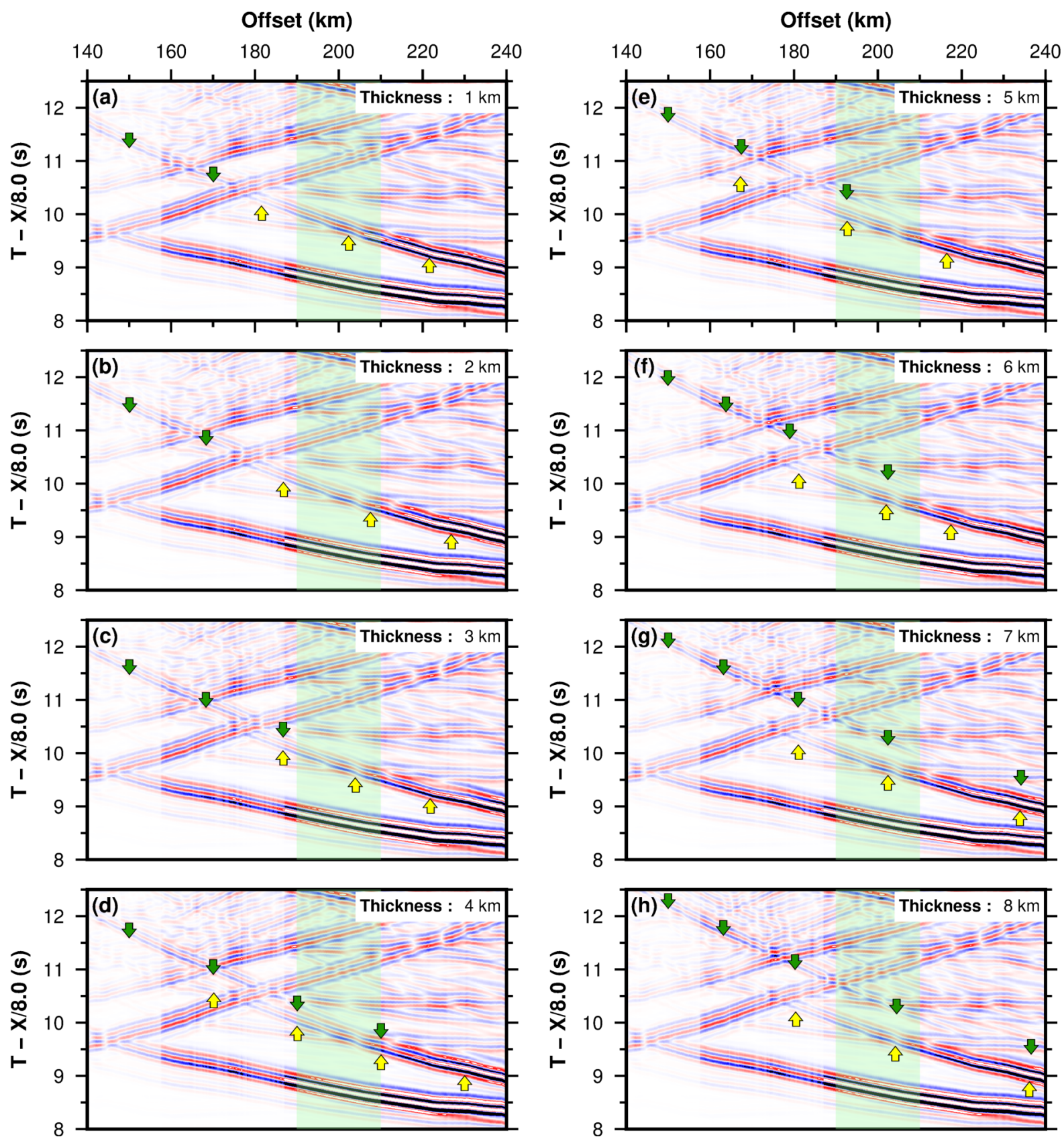

Figure B.12 (a)-(h) Synthetic common receiver gathers for different thicknesses of the anisotropic layer. Yellow and green arrows indicate $\mathrm{R}_{0}$ and $\mathrm{R}_{1}$ wide-angle reflections. Light-green shaded region indicates the offset range where $R_{0}$ is observed to merge with $R_{1}$ in the actual common receiver gather in Figure 4.2a. 

Appendix C

Supplementary Material for Chapter 5 

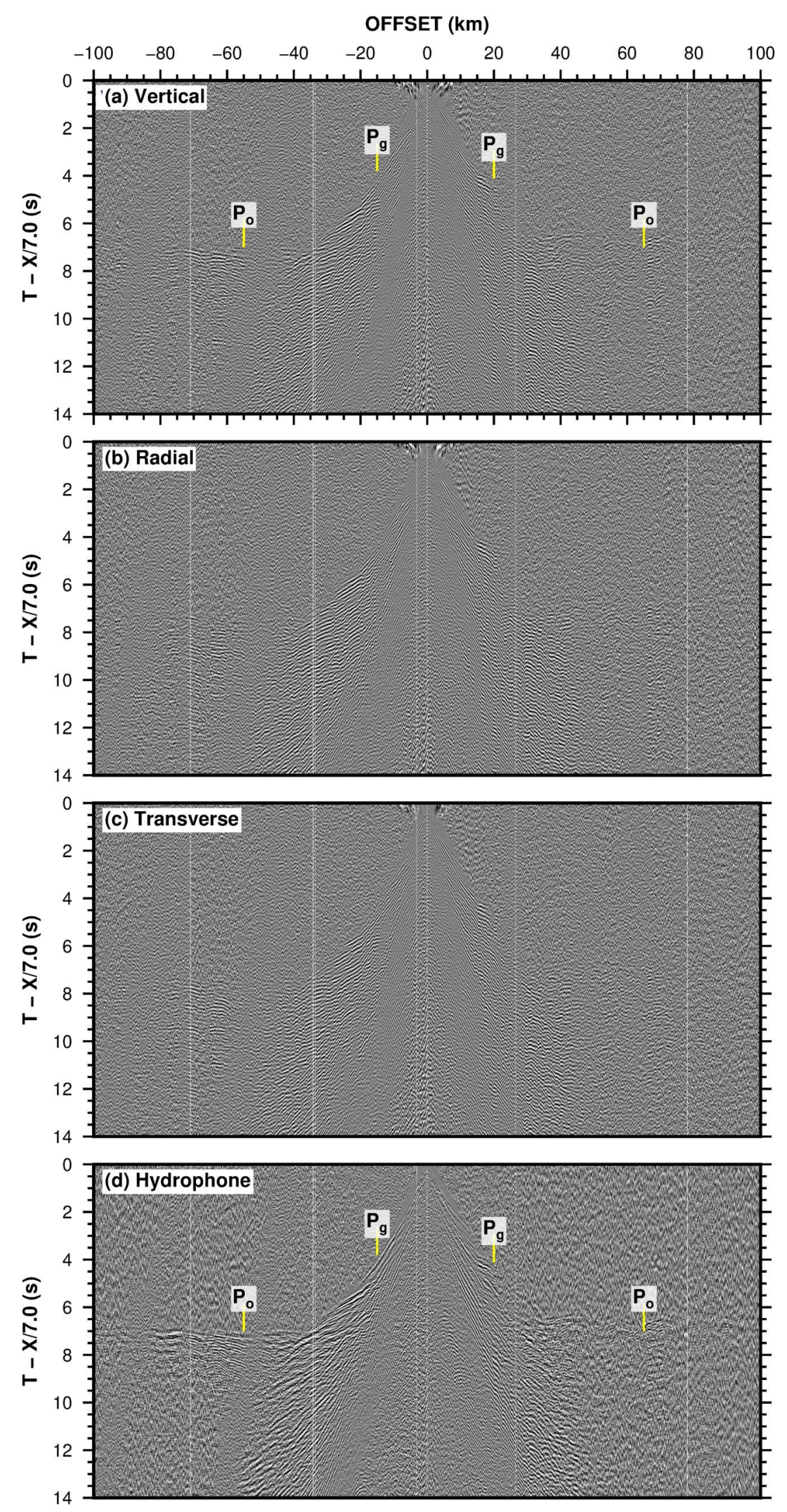

Figure C.1 Common receiver gathers of (a) vertical, (b) radial, (c) transverse and (d) hydrophone components at OBS 331. Note that the travel-time axes are reduced using a reduction velocity of $7.0 \mathrm{~km} / \mathrm{s}$. Identified seismic phases are labelled. $\mathrm{P}_{\mathrm{g}}$-refraction in the upper plate, $\mathrm{P}_{\mathrm{o}}$-refraction in the oceanic crust. Note that converted phases cannot be identified confidently in the radial and transverse components. 


\section{Bibliography}

Aki, K., and P. G. Richards (2002), Quantitative seismology, 2 ed., Sausalito, CA : University Science Books, Sausalito, CA.

Arai, R., S. Kodaira, S. Henrys, N. Bangs, and K. Obana (2020), Three - Dimensional P Wave Velocity Structure of the Northern Hikurangi Margin From the NZ3D Experiment : Evidence for Fault - Bound Anisotropy Journal of Geophysical Research : Solid Earth, Journal of Geophysical Research: Solid Earth, 125, doi:10.1029/2020JB020433.

Babuška, V., and M. Cara (1991), Seismic Anisotropy in the Earth, 1 ed., Kluwer Academic Publishers.

Bangs, N. (2018), SHIRE Project Cruise Report, Tech. rep., University of Texas.

Bannister, S. (1988), Microseismicity and Velocity Structure in the Hawkes Bay Region, New Zealand: Fine Structure of the Subducting Pacific Plate, Geophysical Journal International, 95(1), 45-62.

Barker, D. H. N., R. Sutherland, S. Henrys, and S. Bannister (2009), Geometry of the Hikurangi subduction thrust and upper plate, North Island, New Zealand, Geochemistry, Geophysics, Geosystems, 10(2), doi:10.1029/2008GC002153.

Barker, D. H. N., Van Avendonk, and G. Fujie (2019), Seismogenesis at Hikurangi Integrated Research Experiment (SHIRE) Report of R/V Tangaroa cruise TAN1710, Tech. rep., GNS Science.

Barnes, P. M., G. Lamarche, J. Bialas, S. Henrys, I. Pecher, G. L. Netzeband, J. Greinert, 
J. J. Mountjoy, K. Pedley, and G. Crutchley (2010), Tectonic and geological framework for gas hydrates and cold seeps on the Hikurangi subduction margin, New Zealand, Marine Geology, 272(1-4), 26-48, doi:10.1016/j.margeo.2009.03.012.

Bassett, D., R. Sutherland, and S. Henrys (2014), Slow wavespeeds and fluid overpressure in a region of shallow geodetic locking and slow slip, Hikurangi subduction margin, New Zealand, doi:10.1016/j.epsl.2013.12.021.

Bassett, D., H. Kopp, R. Sutherland, S. Henrys, A. B. Watts, C. Timm, M. Scherwath, I. Grevemeyer, and C. E. J. Ronde (2016), Crustal structure of the Kermadec arc from MANGO seismic refraction profiles, Journal of Geophysical Research: Solid Earth, 121(10), 7514-7546, doi:10.1002/2016JB013194.

Bassett, D., S. Henrys, D. Barker, A. Arnulf, R. Arai, S. Kodaira, H. van Avendonk, N. Bangs, G. Fujie, Y. Yamamoto, K. Obana, and J. Hillman (2018), THE RELATIONSHIP BETWEEN FOREARC STRUCTURE AND GEODETIC LOCKING ALONG THE HIKURANGI MARGIN FROM SHIRE SEISMIC DATA, in Geosciences 2018 Abstract Volume, p. 21, Geoscience Society of New Zealand.

Bean, C. J., and A. Jacob (1990), P-wave anisotropy in the lower lithosphere, Earth and Planetary Science Letters, 99(1-2), 58-65, doi:10.1016/0012-821X(90)90070-E.

Bell, R., R. Sutherland, D. H. N. Barker, S. Henrys, S. Bannister, L. Wallace, and J. Beavan (2010), Seismic reflection character of the Hikurangi subduction interface, New Zealand, in the region of repeated Gisborne slow slip events, Geophysical Journal International, 180(1), 34-48, doi:10.1111/j.1365-246X.2009.04401.x.

Bland, K. J., C. I. Uruski, and M. J. Isaac (2015), Pegasus Basin, eastern New Zealand: A stratigraphic record of subsidence and subduction, ancient and modern, New Zealand Journal of Geology and Geophysics, 58(4), 319-343, doi:10.1080/00288306.2015.1076862.

Blangy, J. P. (1994), AVO in transversely Isotropic media-An overview, GEOPHYSICS, 59(5), 775-781, doi:10.1190/1.1443635. 
Brisbourne, A. M., and G. W. Stuart (1998), Shear-wave velocity structure beneath North Island, New Zealand, from Rayleigh-wave interstation phase velocities, Geophysical Journal International, 133(1), 175-184.

Brudzinski, M. R., C. H. Thurber, B. R. Hacker, and E. R. Engdahl (2007), Global Prevalence of Double Benioff Zones, Science, 316(5830), 1472 LP - 1474, doi:10.1126/ science.1139204.

Cai, C., D. Wiens, W. Shen, and M. Eimer (2018), Water input into the Mariana subduction zone estimated from ocean-bottom seismic data, doi:10.1038/s41586-018-0655-4.

Cape, C. D., S. H. Lamb, P. Vella, P. E. Wells, and D. J. Woodward (1990), Geological structure of Wairarapa Valley, New Zealand, from seismic reflection profiling, Journal of the Royal Society of New Zealand, 20(1), 85-105, doi:10.1080/03036758.1990.10426734.

Castagna, J. P. (1993), Petrophysical imaging using AVO, The Leading Edge, 12(3), 172-178, doi:10.1190/1.1436939.

Castagna, J. P., M. L. Batzle, and R. L. Eastwood (1985), Relationships between compressional-wave and shear-wave velocities in clastic silicate rocks, GEOPHYSICS, 50(4), 571-581, doi:10.1190/1.1441933.

Chadwick, M. P. (1997), The 1991 Hikurangi Margin Seismic Refraction Experiment, Ph.d. thesis, Victoria University of Wellington.

Charvis, P., M. Recq, S. Operto, and D. Brefort (1995), Deep structure of the northern Kerguelen Plateau and hotspot-related activity, Geophysical Journal International, 122(3), 899-924, doi:10.1111/j.1365-246X.1995.tb06845.x.

Chen, M., and J. Tromp (2007), Theoretical and numerical investigations of global and regional seismic wave propagation in weakly anisotropic earth models, Geophysical Journal International, 168(3), 1130-1152, doi:10.1111/j.1365-246X.2006.03218.x. 
Chong, F. K. L. (1982), A Microearthquake Study of the Indian/Pacific Plate Boundary, Central Hawkes Bay, New Zealand, Phd thesis, Victoria University of Wellington.

Chow, B., Y. Kaneko, C. Tape, R. Modrak, and J. Townend (2020), Adjoint Tomography of the North Island, New Zealand Using an Automated Workflow, in Abstract S0630001 presented at 2020 AGU Fall Meeting 1-17 December (online).

Christensen, N. I. (1984), Pore pressure and oceanic crustal seismic structure, Geophysical Journal International, 79(2), 411-423, doi:10.1111/j.1365-246X.1984.tb02232.x.

Christensen, N. I. (1996), Poisson's ratio and crustal seismology, Journal of Geophysical Research: Solid Earth, 101(B2), 3139-3156, doi:10.1029/95JB03446.

Cloos, M. (1993), Lithospheric buoyancy and collisional orogenesis: Subduction of oceanic plateaus, continental margins, island arcs, spreading ridges, and seamounts, GSA Bulletin, 105(6), 715-737, doi:10.1130/0016-7606(1993)105<0715:LBACOS>2.3. $\mathrm{CO} ; 2$.

Coffin, M. F., and O. Eldholm (1994), Large igneous provinces: Crustal structure, dimensions, and external consequences, Reviews of Geophysics, 32(1) , 1-36, doi:10.1029/ 93RG02508.

Contreras-Reyes, E., P. Muñoz-Linford, V. Cortés-Rivas, J. P. Bello-González, J. A. Ruiz, and A. Krabbenhoeft (2019), Structure of the Collision Zone Between the Nazca Ridge and the Peruvian Convergent Margin: Geodynamic and Seismotectonic Implications, Tectonics, 38(9), 3416-3435, doi:10.1029/2019TC005637.

Crampin, S. (1990), The scattering of shear-waves in the crust, pure and applied geophysics, 132(1), 67-91, doi:10.1007/BF00874358.

Davy, B., and R. Wood (1994), Gravity and magnetic modelling of the Hikurangi Plateau, Marine Geology, 118(1), 139-151, doi:https://doi.org/10.1016/0025-3227(94) 90117-1. 
Davy, B., K. Hoernle, and R. Werner (2008), Hikurangi Plateau: Crustal structure, rifted formation, and Gondwana subduction history, Geochemistry, Geophysics, Geosystems, 9(7), doi:10.1029/2007GC001855.

DeMets, C., R. G. Gordon, and D. F. Argus (2010), Geologically current plate motions, Geophysical Journal International, 181(1), 1-80.

Díaz, J., J. Gallart, D. Córdoba, L. Senos, L. Matias, E. SuriÑach, A. Hirn, and P. Maguire (1993), A deep seismic sounding investigation of lithospheric heterogeneity and anisotropy beneath the Iberian Peninsula, Tectonophysics, 221(1), 35-51, doi:10.1016/ 0040-1951(93)90026-G.

Digranes, P., R. Mjelde, S. Kodaira, H. Shimamura, T. Kanazawa, and H. Shiobara (2003), Regional and semi-regional modelling of wide-angle shear waves in OBS data from the Vøring Basin, N. Norway-a comparison, Earth, Planets and Space, 55(2), 65-81, doi:10.1186/BF03351734.

Djeffal, A., I. A. Pecher, S. C. Singh, G. J. Crutchley, and J. Kaipio (2021), Improved imaging of gas hydrate reservoirs and their plumbing system using $2 \mathrm{~d}$ elastic full waveform inversion, Interpretation, pp. 1-61, doi:10.1190/int-2020-0163.1.

Dobrin, M. B. M. B. (1988), Introduction to geophysical prospecting, 4th ed., New York : McGraw-Hill Book Co., New York.

Du, W., C. H. Thurber, M. Reyners, D. Eberhart-Phillips, and H. Zhang (2004), New constraints on seismicity in the Wellington region of New Zealand from relocated earthquake hypocentres, Geophysical Journal International, 158(3), 1088-1102, doi:10. 1111/j.1365-246X.2004.02366.x.

Eberhart-Phillips, D., D.-H. Han, and M. Zoback (1989), Porosity , and Clay Content in Sandstone, Geophysics, 54(1), 82-89.

Eberhart-Phillips, D., M. Reyners, M. Chadwick, and J.-M. Chiu (2005), Crustal het- 
erogeneity and subduction processes: 3-D Vp, Vp/Vs and Q in the southern North Island, New Zealand, Geophysical Journal International, 162(1), 270-288, doi:10.1111/ j.1365-246X.2005.02530.x.

Eberhart-Phillips, D., M. Reyners, S. Bannister, M. Chadwick, and S. Ellis (2010), Establishing a Versatile 3-D Seismic Velocity Model for New Zealand, Seismological Research Letters, 81(6), 992-1000.

Eberhart-Phillips, D., S. Bannister, and M. Reyners (2017), Deciphering the 3-D distribution of fluid along the shallow Hikurangi subduction zone using P- and Swave attenuation, Geophysical Journal International, 211(2), 1032-1045, doi:10.1093/ gji/ggx348.

Eberhart-Phillips, D., S. Bannister, M. Reyners, and S. Henrys (2020), New Zealand Wide model 2.2 seismic velocity and Qs and Qp models for New Zealand [Dataset], Zenodo, doi:10.5281/ZENODO.3779523.

Eccles, J. D. (2008), Shear Wave Analysis of Volcanic Rifted Continental Margins in the North Atlantic, Phd, University of Cambridge.

Eccles, J. D., R. S. White, and P. A. F. Christie (2009), Identification and inversion of converted shear waves: case studies from the European North Atlantic continental margins, Geophysical Journal International, 179(1), 381-400, doi:10.1111/j.1365-246X. 2009.04290.x.

Emry, E. L., and D. A. Wiens (2015), Incoming plate faulting in the Northern and Western Pacific and implications for subduction zone water budgets, Earth and Planetary Science Letters, 414(C), 176-186, doi:10.1016/j.epsl.2014.12.042.

Evanzia, D., T. Wilson, M. K. Savage, S. Lamb, and H. Hirschberg (2017), Stress Orientations in a Locked Subduction Zone at the Southern Hikurangi Margin, New Zealand, Journal of Geophysical Research: Solid Earth, 122(10), 7895-7911, doi:10.1002/ 2017JB013998. 
Evanzia, D., S. Lamb, M. K. Savage, and T. Stern (2018), Illumination of deformation by bending stresses and slab pull within the Southern Hikurangi Double Benioff Zone, doi:10.1080/00288306.2018.1532439.

Faccenda, M., T. V. Gerya, and L. Burlini (2009), Deep slab hydration induced by bending-related variations in tectonic pressure, Nature Geoscience, 2, 790.

Fagereng, A., and S. Ellis (2009), On factors controlling the depth of interseismic coupling on the Hikurangi subduction interface, New Zealand, Earth and Planetary Science Letters, 278(1), 120-130, doi:https://doi.org/10.1016/j.eps1.2008.11.033.

Farfour, M., and W. J. Yoon (2016), A review on multicomponent seismology: A potential seismic application for reservoir characterization, Journal of Advanced Research, 7(3), 515-524, doi:https://doi.org/10.1016/j.jare.2015.11.004.

Fischer, K. M., H. A. Ford, D. L. Abt, and C. A. Rychert (2010), The LithosphereAsthenosphere Boundary, Annual Review of Earth and Planetary Sciences, 38(1), 551-575, doi:10.1146/annurev-earth-040809-152438.

Flinn, E. A. (1965), Signal analysis using rectilinearity and direction of particle motion, Proceedings of the IEEE, 53(12), 1874-1876, doi:10.1109/PROC.1965.4462.

Florez, M. A., and G. A. Prieto (2019), Controlling Factors of Seismicity and Geometry in Double Seismic Zones, Geophysical Research Letters, 46(8), 4174-4181, doi:10.1029/ 2018GL081168.

Fuchs, K. (1979), Structure, Physical Properties and Lateral Heterogeneities of the Subcrustal Lithosphere from Long-range Deep Seismic Sounding Observations on Continents, Tectonophysics, 56, 1-15.

Fujie, G., S. Kodaira, Y. Kaiho, Y. Yamamoto, T. Takahashi, S. Miura, and T. Yamada (2018), Controlling factor of incoming plate hydration at the north-western Pacific margin, Nature Communications, 9(1), doi:10.1038/s41467-018-06320-z. 
Furumoto, A. S., J. P. Webb, M. E. Odegard, and D. M. Hussong (1976), Seismic studies on the Ontong Java Plateau, 1970, Tectonophysics, 34(1), 71-90, doi:https://doi.org/ 10.1016/0040-1951(76)90177-3.

Galea, P. (1992), Observations of very high P-velocities in the subducted slab, New Zealand, and their relation with the slab geometry, Geophysical Journal International, 110(2), 238-250.

Galea, P. (1993), Upper mantle anisotropy in the S.W. Pacific from earthquake traveltime analysis, Physics of the Earth and Planetary Interiors, 76(3), 229-239, doi:https: //doi.org/10.1016/0031-9201(93)90015-2.

Garcia, E. S. M., D. T. Sandwell, and D. Bassett (2019), Outer trench slope flexure and faulting at Pacific basin subduction zones, Geophysical Journal International, 218(1), 708-728, doi:10.1093/gji/ggz155.

Geuzaine, C., and J. F. Remacle (2009), Gmsh: A 3-D finite element mesh generator with built-in pre- and post-processing facilities, International Journal for Numerical Methods in Engineering, 79(11), 1309-1331, doi:10.1002/nme.2579.

Gledhill, K., and D. Gubbins (1996), SKS splitting and the seismic anisotropy of the mantle beneath the Hikurangi subduction zone, New Zealand, Physics of the Earth and Planetary Interiors, 95(3), 227-236, doi:https://doi.org/10.1016/0031-9201(95) 03118-9.

Graham, K. M., M. K. Savage, R. Arnold, H. J. Zal, T. Okada, Y. Iio, and S. Matsumoto (2020), Spatio-temporal Analysis of Seismic Anisotropy Associated with the Cook Strait and Kaikōura Earthquake Sequences in New Zealand, Geophysical Journal International, doi:10.1093/gji/ggaa433.

Grevemeyer, I., E. R. Flueh, C. Reichert, J. Bialas, D. Kläschen, and C. Kopp (2001), Crustal architecture and deep structure of the Ninetyeast Ridge hotspot trail from 
active-source ocean bottom seismology, Geophysical Journal International, 144(2), 414-431, doi:10.1046/j.0956-540X.2000.01334.x.

Grevemeyer, I., C. R. Ranero, E. R. Flueh, D. Kläschen, and J. Bialas (2007), Passive and active seismological study of bending-related faulting and mantle serpentinization at the Middle America trench, Earth and Planetary Science Letters, 258(3), 528-542, doi:https://doi.org/10.1016/j.epsl.2007.04.013.

Grevemeyer, I., R. Cesar, and M. Ivandic (2018), Structure of oceanic crust and serpentinization at subduction trenches, Geosphere, 14(2), 395-418, doi:10.1130/GES01537.1.

Grobys, J. W., K. Gohl, and G. Eagles (2008), Quantitative tectonic reconstructions of Zealandia based on crustal thickness estimates, Geochemistry, Geophysics, Geosystems, 9(1), doi:10.1029/2007GC001691.

Guillot, S., S. Schwartz, B. Reynard, P. Agard, and C. Prigent (2015), Tectonic significance of serpentinites, Tectonophysics, 646,1-19, doi:10.1016/j.tecto.2015.01.020.

Hacker, B. R. (1996), Eclogite formation and the rheology, buoyancy, seismicity, and H2O content of oceanic crust, vol. 96, 337-346 pp., American Geophysical Union, doi:10. 1029/GM096p0337.

Hacker, B. R., S. M. Peacock, G. A. Abers, and S. D. Holloway (2003), Subduction factory 2. Are intermediate-depth earthquakes in subducting slabs linked to metamorphic dehydration reactions?, Journal of Geophysical Research: Solid Earth, 108(B1), n/a-n/a, doi:10.1029/2001JB001129.

Haines, A. J. (1979), Seismic wave velocities in the uppermost mantle beneath New Zealand, New Zealand Journal of Geology and Geophysics, 22(2), 245-257, doi:10.1080/ 00288306.1979.10424223.

Hampel, A., N. Kukowski, J. Bialas, C. Huebscher, and R. Heinbockel (2004), Ridge 
subduction at an erosive margin: The collision zone of the Nazca Ridge in southern Peru, Journal of Geophysical Research: Solid Earth, 109(B2), doi:10.1029/2003jb002593.

Hayes, G. P., G. L. Moore, D. E. Portner, M. Hearne, H. Flamme, M. Furtney, and G. M. Smoczyk (2018), Slab2, a comprehensive subduction zone geometry model, Science, 362(6410), 58 LP - 61, doi:10.1126/science.aat4723.

Henrys, S., M. Reyners, I. Pecher, S. Bannister, Y. Nishimura, and G. Maslen (2006), Kinking of the subducting slab by escalator normal faulting beneath the North Island of New Zealand, Geology, 34(9), 777-780.

Henrys, S., A. Wech, R. Sutherland, T. Stern, M. Savage, H. Sato, K. Mochizuki, T. Iwasaki, D. Okaya, A. Seward, B. Tozer, J. Townend, E. Kurashimo, T. Iidaka, and T. Ishiyama (2013), SAHKE geophysical transect reveals crustal and subduction zone structure at the southern Hikurangi margin, New Zealand, Geochemistry, Geophysics, Geosystems, 14(7), 2063-2083, doi:10.1002/ggge.20136.

Herath, P., T. A. Stern, M. K. Savage, D. Bassett, S. Henrys, and C. Boulton (2020), Hydration of the crust and upper mantle of the Hikurangi Plateau as it subducts at the southern Hikurangi margin, Earth and Planetary Science Letters, 541, 116,271, doi:https://doi.org/10.1016/j.epsl.2020.116271.

Hess, H. H. (1964), Seismic Anisotropy of the Uppermost Mantle under Oceans, Nature, 203(4945), 629-631, doi:10.1038/203629a0.

Hines, B., D. Kulhanek, C. Hollis, C. Atkins, and H. Morgans (2013), Paleocene-Eocene Stratigraphy and Paleoenvironment at Tora, Southeast Wairarapa, New Zealand, New Zealand Journal of Geology and Geophysics, 56, 243-262.

Hirn, A. (1977), Anisotropy in the continental upper mantle: possible evidence from explosion seismology, Geophysical Journal of the Royal Astronomical Society, 49(1) ,49-58, doi:10.1111/j.1365-246X.1977.tb03700.x. 
Hirschberg, H. P., S. Lamb, and M. K. Savage (2019), Strength of an obliquely convergent plate boundary: Lithospheric stress magnitudes and viscosity in New Zealand, Geophysical Journal International, 216(2), 1005-1024, doi:10.1093/gji/ggy477.

Hochmuth, K., K. Gohl, and G. Uenzelmann-Neben (2015), Playing jigsaw with Large Igneous Provinces-A plate tectonic reconstruction of Ontong Java Nui, West Pacific, Geochemistry, Geophysics, Geosystems, 16(11), 3789-3807, doi:10.1002/2015GC006036.

Hochmuth, K., K. Gohl, G. Uenzelmann-Neben, and R. Werner (2019), Multiphase magmatic and tectonic evolution of a large igneous province - Evidence from the crustal structure of the Manihiki Plateau, western Pacific, Tectonophysics, 750, 434-457, doi:https://doi.org/10.1016/j.tecto.2018.11.014.

Hoernle, K., F. Hauff, P. van den Bogaard, R. Werner, N. Mortimer, J. Geldmacher, D. Garbe-Schoenberg, and B. Davy (2010), Age and geochemistry of volcanic rocks from the Hikurangi and Manihiki oceanic plateaus, Geochimica et Cosmochimica Acta, 74(24), 7196-7219, doi:10.1016/j.gca.2010.09.030.

Igel, H. (2017), Computational seismology : a practical introduction, first edit ed., Oxford : Oxford University Press.

Jordan, T. (1978), Composition and development of the continental tectosphere, vol. 274, doi: $10.1038 / 274544 a 0$.

Joyner, W. B. (2000), Strong motion from surface waves in deep sedimentary basins, Bulletin of the Seismological Society of America, 90(6 SUPPL.), 95-112, doi:10.1785/ 0120000505.

Jung, H., and S.-i. Karato (2001), Water-Induced Fabric Transitions in Olivine, Science, 293(5534), 1460 LP - 1463.

Kandilarov, A., R. Mjelde, E. Flueh, and R. B. Pedersen (2015), Vp/Vs-ratios and 
anisotropy on the northern Jan Mayen Ridge, North Atlantic, determined from ocean bottom seismic data, Polar Science, 9(3), 293-310, doi:10.1016/j.polar.2015.06.001.

Kaneko, Y., Y. Ito, B. Chow, L. M. Wallace, C. Tape, R. Grapenthin, E. D'Anastasio, S. Henrys, and R. Hino (2019), Ultra-long duration of seismic ground motion arising from a thick, low velocity sedimentary wedge, Journal of Geophysical Research: Solid Earth, 0(ja), doi:10.1029/2019JB017795.

Karato, S.-i., H. Jung, I. Katayama, and P. Skemer (2008), Geodynamic Significance of Seismic Anisotropy of the Upper Mantle: New Insights from Laboratory Studies, $A n-$ nual Review of Earth and Planetary Sciences, 36(1), 59-95, doi:10.1146/annurev.earth. 36.031207.124120.

Katayama, I., H. Jung, and S.-i. Karato (2004), New type of olivine fabric from deformation experiments at modest water content and low stress, Geology, 32(12), 1045-1048, doi:10.1130/G20805.1.

Kawakatsu, H., P. Kumar, Y. Takei, M. Shinohara, T. Kanazawa, E. Araki, and K. Suyehiro (2009), Seismic evidence for sharp lithosphere-asthenosphere boundaries of oceanic plates, Science, 324(5926), 499-502, doi:10.1126/science.1169499.

Kayal, J. R., and E. G. C. Smith (1984), Upper mantle P-wave velocities in the southeast North Island, New Zealand, Tectonophysics, 104(1), 115-125, doi:https://doi.org/10. 1016/0040-1951(84)90105-7.

Kearey, P., M. Brooks, and I. Hill (2002), An Introduction to Geophysical Exploration, 3 ed., Blackwell Science.

Kennett, B. L. N., and E. R. Engdahl (1991), Traveltimes for global earthquake location and phase identification, Geophysical Journal International, 105(2), 429-465, doi: 10.1111/j.1365-246X.1991.tb06724.x. 
Kerr, A. C. (2005), Oceanic LIPs: The Kiss of Death, Elements, 1(5), 289-292, doi:10. 2113/gselements.1.5.289.

Kerr, A. C. (2014), Oceanic Plateaus, in Encyclopedia of Marine Geosciences, edited by J. Harff, M. Meschede, S. Petersen, and J. Thiede, pp. 1-15, Springer Netherlands, Dordrecht, doi:10.1007/978-94-007-6644-0_21-1.

Kobussen, A. F., N. I. Christensen, and H. Thybo (2006), Constraints on seismic velocity anomalies beneath the Siberian craton from xenoliths and petrophysics, Tectonophysics, 425(1), 123-135, doi:10.1016/j.tecto.2006.07.008.

Kodaira, S., M. Bellenberg, T. Iwasaki, T. Kanazawa, H. B. Hirschleber, and H. Shimamura (1996), Vp/ Vs ratio structure of the Lofoten continental margin, northern Norway, and its geological implications, Geophysical Journal International, 124(3), 724-740, doi:10.1111/j.1365-246X.1996.tb05634.x.

Komatitsch, D., and J. Tromp (1999), Introduction to the spectral element method for three-dimensional seismic wave propagation, Geophysical Journal International, 139(3), 806-822, doi:10.1046/j.1365-246X.1999.00967.x.

Komatitsch, D., G. Erlebacher, D. Göddeke, and D. Michéa (2010), High-order finiteelement seismic wave propagation modeling with MPI on a large GPU cluster, Journal of Computational Physics, 229(20), 7692-7714, doi:10.1016/j.jcp.2010.06.024.

Korenaga, J. (2017), On the extent of mantle hydration caused by plate bending, Earth and Planetary Science Letters, 457, 1-9, doi:10.1016/j.eps1.2016.10.011.

Kreemer, C. (2009), Absolute plate motions constrained by shear wave splitting orientations with implications for hot spot motions and mantle flow, Journal of Geophysical Research: Solid Earth, 114(10), 1-18, doi:10.1029/2009JB006416.

Kumar, P., X. Yuan, M. R. Kumar, R. Kind, X. Li, and R. K. Chadha (2007), The rapid drift of the Indian tectonic plate, Nature, 449(7164), 894-897, doi:10.1038/nature06214. 
Lee, B. M. W. (2003), Velocity Ratio and its Application to Predicting Velocities, U.S. Geological Survey Bulletin, 2197, 19.

Litchfield, N., R. Van Dissen, R. Sutherland, P. Barnes, S. Cox, R. Norris, R. Beavan, R. Langridge, P. Villamor, K. Berryman, M. Stirling, A. Nicol, S. Nodder, G. Lamarche, D. Barrell, J. Pettinga, T. Little, N. Pondard, J. Mountjoy, and K. Clark (2013), A model of active faulting in New Zealand, New Zealand Journal of Geology and Geophysics, 57(1), 1-25, doi:10.1080/00288306.2013.854256.

Love, H., M. LeGood, G. Stuart, M. Reyners, D. E. Eberhart-Phillips, and D. Gubbins (2015), Fast P -wave precursors in New Zealand: high velocity material associated with the subducted Hikurangi Plateau, Geophysical Journal International, 202(2), 1223-1240, doi:10.1093/gji/ggv205.

Lowrie, W. (2007), Fundamentals of Geophysics, second ed., Cambridge University Press.

Luckie, T., A. Gase, K. Jacobs, M. C. White, S. Henrys, D. Okaya, H. Van Avendonk, N. Bangs, D. Barker, D. Bassett, S. Kodaira, R. Arai, G. Fujie, and Y. Yamamoto (2020), P-wave velocity structure of the northern Hikurangi margin from travel time tomography, in Abstract T017-0010 presented at 2020 AGU Fall Meeting 1-17 December (online).

Lund, C. E. (1979), The fine structure of the lower lithosphere underneath the Blue Road profile in northern Scandinavia, Tectonophysics, 56(1-2), 111-122, doi:10.1016/ 0040-1951(79)90017-9.

Mainprice, D., R. Hielscher, and H. Schaeben (2011), Calculating anisotropic physical properties from texture data using the MTEX open-source package, Geological Society of London Special Publications, 360, 175-192, doi:10.1144/SP360.10.

Marson-Pidgeon, K., and M. K. Savage (2004), Shear-wave splitting variations across an array in the southern North Island, New Zealand, Geophysical Research Letters, 31(21), doi:10.1029/2004GL021190. 
Mehouachi, F., and S. C. Singh (2018), Water-rich sublithospheric melt channel in the equatorial Atlantic Ocean, Nature Geoscience, 11(1), 65-69, doi:10.1038/ s41561-017-0034-z.

Miller, N. C., and D. Lizarralde (2016), Finite-frequency wave propagation through outer rise fault zones and seismic measurements of upper mantle hydration, Geophysical Research Letters, 43(15), 7982-7990, doi:10.1002/2016GL070083.

Mitchell, J., K. Mackay, H. Neil, E. Mackay, A. Pallentin, and P. Notman (2012), Undersea New Zealand, 1:5,000,000. NIWA Chart, Miscellaneous Series No. 92.

Miura, S., K. Suyehiro, M. Shinohara, N. Takahashi, E. Araki, and A. Taira (2004), Seismological structure and implications of collision between the Ontong Java Plateau and Solomon Island Arc from ocean bottom seismometer-airgun data, Tectonophysics, 389(3), 191-220, doi:https://doi.org/10.1016/j.tecto.2003.09.029.

Mochizuki, K., R. Sutherland, S. Henrys, D. Bassett, H. van Avendonk, R. Arai, S. Kodaira, G. Fujie, Y. Yamamoto, N. Bangs, and D. Barker (2019), Plumes, Recycling of depleted continental mantle by subduction and Hikurangi Plateau Large Igneous Province, southwest Pacific, Geology.

Moreno, M., C. Haberland, O. Oncken, A. Rietbrock, S. Angiboust, and O. Heidbach (2014), Locking of the Chile subduction zone controlled by fluid pressure before the 2010 earthquake, Nature Geoscience, 7(4), 292, doi:10.1038/ngeo2102.

Mortimer, N. (2004), New Zealand's Geological Foundations, Gondwana Research, 7(1), 261-272, doi:https://doi.org/10.1016/S1342-937X(05)70324-5.

Mountjoy, J. J., and P. M. Barnes (2011), Active upper plate thrust faulting in regions of low plate interface coupling, repeated slow slip events, and coastal uplift: Example from the Hikurangi Margin, New Zealand, Geochemistry, Geophysics, Geosystems, 12(1), 1-26, doi:10.1029/2010GC003326. 
Naif, S., K. Key, S. Constable, and R. L. Evans (2013), Melt-rich channel observed at the lithosphere-asthenosphere boundary, Nature, 495(7441), 356-359, doi:10.1038/ nature11939.

Naif, S., K. Key, S. Constable, and R. L. Evans (2015), Water-rich bending faults at the Middle America Trench, Geochemistry, Geophysics, Geosystems, 16(8), 2582-2597, doi: 10.1002/2015GC005927.

Okaya, D., T. Stern, S. Holbrook, H. van Avendonk, F. Davey, and S. Henrys (2003), Imaging a plate boundary using double-sided onshore-offshore seismic profiling, The Leading Edge, 22(3), 256-260.

Pancha, A. (1994), Gravity survey of the Eketahuna Basin, North Island, New Zealand, Ph.D. thesis, Victoria University of Wellington.

Peacock, S., G. K. Westbrook, and G. Bais (2010), S-wave velocities and anisotropy in sediments entering the Nankai subduction zone, offshore Japan, Geophysical Journal International, 180(2), 743-758, doi:10.1111/j.1365-246X.2009.04430.x.

Peacock, S. M. (2001), Are the lower planes of double seismic zones caused by serpentine dehydration in subducting oceanic mantle?, Geology, 29(4), 299-302, doi: 10.1130/0091-7613(2001)029<0299:ATLPOD>2.0.CO;2.

Perelberg, A. I., and S. C. Hornbostel (1994), Applications of seismic polarization analysis, GEOPHYSICS, 59(1), 119-130, doi:10.1190/1.1443522.

Planert, L., H. Kopp, E. Lueschen, C. Mueller, E. R. Flueh, A. Shulgin, Y. Djajadihardja, and A. Krabbenhoeft (2010), Lower plate structure and upper plate deformational segmentation at the Sunda-Banda arc transition, Indonesia, Journal of Geophysical Research: Solid Earth, 115(8), 1-25, doi:10.1029/2009JB006713.

Pulford, A., M. Savage, and T. Stern (2003), Absent anisotropy: The paradox of the 
Southern Alps orogen, Geophysical Research Letters, 30(20), doi:https://doi.org/10. 1029/2003GL017758.

Raitt, R. W., G. G. Shor, G. B. Morris, and H. K. Kirk (1971), Mantle anisotropy in the Pacific Ocean, Tectonophysics, 12(3), 173-186, doi:https://doi.org/10.1016/ 0040-1951(71)90002-3.

Ranero, C. R., and V. Sallarès (2004), Geophysical evidence for hydration of the crust and mantle of the Nazca plate during bending at the north Chile trench, Geology, $32(7), 549-552$.

Ranero, C. R., J. Phipps Morgan, K. McIntosh, and C. Reichert (2003), Bending-related faulting and mantle serpentinization at the Middle America trench, Nature, 425, 367.

Reyners, M. (2012), The central role of the Hikurangi Plateau in the Cenozoic tectonics of New Zealand and the Southwest Pacific, Earth and Planetary Science Letters, 361, doi:10.1016/j.epsl.2012.11.010.

Reyners, M., and D. Eberhart-Phillips (2009), Small earthquakes provide insight into plate coupling and fluid distribution in the Hikurangi subduction zone, New Zealand, Earth and Planetary Science Letters, 282(1), 299-305, doi:https://doi.org/10. 1016/j.eps1.2009.03.034.

Reyners, M., D. Eberhart-Phillips, and S. Bannister (2011), Tracking repeated subduction of the Hikurangi Plateau beneath New Zealand, Earth and Planetary Science Letters, 311(1), 165-171, doi:10.1016/j.eps1.2011.09.011.

Riefstahl, F., K. Gohl, B. Davy, K. Hoernle, N. Mortimer, C. Timm, R. Werner, and K. Hochmuth (2020), Cretaceous intracontinental rifting at the southern Chatham Rise margin and initialisation of seafloor spreading between Zealandia and Antarctica, Tectonophysics, 776(May 2019), 228,298, doi:10.1016/j.tecto.2019.228298. 
Robinson, R. (1986), Seismicity, structure and tectonics of the Wellington region, New Zealand, Geophysical Journal International, 87(2), 379-409.

Rychert, C. A., K. M. Fischer, and S. Rondenay (2005), A sharp lithosphereasthenosphere boundary imaged beneath eastern North America, Nature, 436(7050), 542-545, doi:10.1038/nature03904.

Rychert, C. A., P. M. Shearer, and K. M. Fischer (2010), Scattered wave imaging of the lithosphere-asthenosphere boundary, Lithos, 120(1-2), 173-185, doi:10.1016/j.lithos. 2009.12.006.

Savage, M. K. (1999), Seismic anisotropy and mantle deformation: What have we learned from shear wave splitting?, Reviews of Geophysics, 37(1) , 65-106, doi:10.1029/ 98RG02075.

Savage, M. K., J. Park, and H. Todd (2007), Velocity and anisotropy structure at the Hikurangi subduction margin, New Zealand from receiver functions, Geophysical Journal International, 168(3), 1034-1050, doi:10.1111/j.1365-246X.2006.03086.x.

Savage, M. K., A. Wessel, N. A. Teanby, and A. W. Hurst (2010), Automatic measurement of shear wave splitting and applications to time varying anisotropy at Mount Ruapehu volcano, New Zealand, Journal of Geophysical Research: Solid Earth, 115(12), 1-17, doi:10.1029/2010JB007722.

Schuberth, B. (2003), The Spectral Element Method for Seismic Wave Propagation, Ph.D. thesis, Ludwig-Maximilians University, Munich.

Seward, A., S. Henrys, M. Henderson, R. Sutherland, T. Stern, M. Savage, K. Mochizuki, H. Sato, T. Iwasaki, C. Kennedy, and D. G. Bassett (2010), Seismic Array HiKurangi Experiment (SAHKE) Field Acquisition Report, Tech. rep., GNS Science.

Shearer, P. M. (2009), Introduction to Seismology, 2 ed., Cambridge University Press, Cambridge, doi:DOI:10.1017/CBO9780511841552. 
Shillington, D., A. Bécel, M. Nedimovic, H. Kuehn, S. Webb, G. Abers, K. Keranen, J. Li, M. Delescluse, and G. Mattei-Salicrup (2015), Link between plate fabric, hydration and subduction zone seismicity in Alaska, Nature Geoscience, 8(12), 961-964, doi:10. 1038/ngeo2586.

Shulgin, A., H. Kopp, C. Mueller, L. Planert, E. Lueschen, E. R. Flueh, and Y. Djajadihardja (2011), Structural architecture of oceanic plateau subduction offshore Eastern Java and the potential implications for geohazards, Geophysical Journal International, 184(1), 12-28, doi:10.1111/j.1365-246X.2010.04834.x.

Silver, P. G., and W. W. Chan (1988), Implications for continental structure and evolution from seismic anisotropy, Nature, 335(6185), 34-39, doi:10.1038/335034a0.

Stein, S., and M. Wysession (2003), An Introduction to Seismology, Earthquakes and Earth Structure, 512 pp., Blackwell Publishing.

Stern, T., and A. Benson (2011), Wide-angle seismic imaging beneath an andesitic arc: Central North Island, New Zealand, Journal of Geophysical Research: Solid Earth, 116(B9), doi:10.1029/2011JB008337.

Stern, T., S. A. Henrys, D. Okaya, J. N. Louie, M. K. Savage, S. Lamb, H. Sato, R. Sutherland, and T. Iwasaki (2015), A seismic reflection image for the base of a tectonic plate, Nature, 518(7537), 85, doi:10.1038/nature14146.

Stern, T., S. Lamb, K. Gohl, K. Hochmuth, K. Mochizuki, J. D. Moore, P. Herath, and D. Okaya (2018), HIGH P-WAVE SPEEDS IN THE UPPER MANTLE AND THEIR POSSIBLE ASSOCIATION WITH SUPER PLUMES, in Abstract (T51I-0278) presented at 2018 AGU Fall Meeting 10-14 Dec, Washington, D.C.

Stern, T., S. Lamb, J. D. Moore, J. D. Moore, D. Okaya, and K. Hochmuth (2020), High mantle seismic P-wave speeds as a signature for gravitational spreading of superplumes, Science Advances, 6(22), doi:10.1126/sciadv.aba7118. 
Storchak, D. A., J. Schweitzer, and P. Bormann (2011), Seismic Phase Names: IASPEI Standard, in Encyclopedia of Solid Earth Geophysics, edited by H. K. Gupta, pp. 1162-1173, Springer Netherlands, Dordrecht, doi:10.1007/978-90-481-8702-7_11.

Tanton, L. T. E., and B. H. Hager (2000), Melt intrusion as a trigger for lithospheric foundering and the eruption of the Siberian flood basalts, Geophysical Research Letters, 27(23), 3937-3940, doi:10.1029/2000GL011751.

Taylor, B. (2006), The single largest oceanic plateau: Ontong Java-Manihiki-Hikurangi, Earth and Planetary Science Letters, 241(3), 372-380, doi:https://doi.org/10.1016/j.epsl. 2005.11.049.

Thomsen, L. (1986), Weak elastic anisotropy., Geophysics, 51(10), 1954-1966, doi:10. 1190/1.1442051.

Tommasi, A. (1998), Forward modeling of the development of seismic anisotropy in the upper mantle, Earth and Planetary Science Letters, 160(1-2), 1-13, doi:10.1016/ S0012-821X(98)00081-8.

Tommasi, A., A. Vauchez, and R. Russo (1996), Seismic anisotropy in ocean basins: Resistive drag of the sublithospheric mantle?, Geophysical Research Letters, 23(21), 2991-2994, doi:10.1029/96GL02891.

Townend, J., S. Sherburn, R. Arnold, C. Boese, and L. Woods (2012), Three-dimensional variations in present-day tectonic stress along the Australia-Pacific plate boundary in New Zealand, Earth and Planetary Science Letters, 353-354, 47-59, doi:10.1016/j.epsl. 2012.08.003.

Tozer, B. (2013), Crustal Structure of Wanganui Basin: Implications for Back-Arc Formation, Ph.D. thesis, Victoria University of Wellington.

Tozer, B., T. Stern, S. L. Lamb, and S. A. Henrys (2017), Crust and upper-mantle structure of Wanganui Basin and southern Hikurangi margin, North Island, New Zealand 
as revealed by active source seismic data, Geophysical Journal International, 211(2), 718-740.

Tromp, J., D. Komatitsch, and Q. Liu (2008), Spectral-element and adjoint methods in seismology, Communications in Computational Physics, 3(1), 1-32.

Tsuji, T., J. Dvorkin, G. Mavko, N. Nakata, T. Matsuoka, A. Nakanishi, S. Kodaira, and O. Nishizawa (2011), VP/VS ratio and shear-wave splitting in the Nankai Trough seismogenic zone: Insights into effective stress, pore pressure, and sediment consolidation, Geophysics, 76(3), doi:10.1190/1.3560018.

Van Avendonk, H. J. A., W. Holbrook, D. Lizarralde, and P. Denyer (2011), Structure and serpentinization of the subducting Cocos plate offshore Nicaragua and Costa Rica, Geochemistry, Geophysics, Geosystems, 12(6), doi:10.1029/2011GC003592.

Walcott, R. I. (1984), Reconstructions of the New Zealand region for the Neogene, Palaeogeography, Palaeoclimatology, Palaeoecology, 46(1), 217-231, doi:10.1016/ 0031-0182(84)90035-X.

Wallace, L. M. (2020), Slow Slip Events in New Zealand, Annual Review of Earth and Planetary Sciences, 48, 175-203, doi:10.1146/annurev-earth-071719-055104.

Wallace, L. M., J. Beavan, R. McCaffrey, and D. Darby (2004), Subduction zone coupling and tectonic block rotations in the North Island, New Zealand, Journal of Geophysical Research: Solid Earth, 109(12), 1-21, doi:10.1029/2004JB003241.

Wallace, L. M., M. Reyners, U. Cochran, S. Bannister, P. M. Barnes, K. Berryman, G. Downes, D. Eberhart-Phillips, A. Fagereng, S. Ellis, A. Nicol, R. McCaffrey, R. J. Beavan, S. Henrys, R. Sutherland, D. H. Barker, N. Litchfield, J. Townend, R. Robinson, R. Bell, K. Wilson, and W. Power (2009), Characterizing the seismogenic zone of a major plate boundary subduction thrust: Hikurangi Margin, New Zealand, Geochemistry, Geophysics, Geosystems, 10(10), doi:10.1029/2009GC002610. 
Wallace, L. M., J. Beavan, S. Bannister, and C. Williams (2012a), Simultaneous long-term and short-term slow slip events at the Hikurangi subduction margin, New Zealand: Implications for processes that control slow slip event occurrence, duration, and migration, Journal of Geophysical Research: Solid Earth, 117(B11), n/a-n/a, doi:10.1029/ 2012JB009489.

Wallace, L. M., P. Barnes, J. Beavan, R. Van Dissen, N. Litchfield, J. Mountjoy, R. Langridge, G. Lamarche, and N. Pondard (2012b), The kinematics of a transition from subduction to strike-slip: An example from the central New Zealand plate boundary, Journal of Geophysical Research: Solid Earth, 117(B2), n/a-n/a, doi:10.1029/ 2011JB008640.

White, R. S. (2012), Wide-angle refraction and reflection, in Regional Geology and Tectonics: Principles of Geologic Analysis, edited by D. G. Roberts, A. W. B. T. R. G. Bally, and T. P. of Geologic Analysis, chap. 11, pp. 310-328, Elsevier, Amsterdam, doi: https://doi.org/10.1016/B978-0-444-53042-4.00011-X.

White, R. S., D. McKenzie, and K. R. O’Nions (1992), Oceanic crustal thickness from seismic measurements and rare earth element inversions, Journal of Geophysical Research, 97(B13), 19,683, doi:10.1029/92JB01749.

Williams, C. A., D. Eberhart-Phillips, S. Bannister, D. H. N. Barker, S. Henrys, M. Reyners, and R. Sutherland (2013), Revised interface geometry for the Hikurangi subduction zone, New Zealand, Seismological Research Letters, 84(6), 1066-1073, doi: $10.1785 / 0220130035$.

Wood, R., and B. Davy (1994), The Hikurangi Plateau, Marine Geology, 118(1), 153-173, doi:https://doi.org/10.1016/0025-3227(94)90118-X.

Wüstefeld, A., G. Bokelmann, C. Zaroli, and G. Barruol (2008), SplitLab: A shear-wave splitting environment in Matlab, Computers and Geosciences, 34(5), 515-528, doi:10. 1016/j.cageo.2007.08.002. 
Yarce, J., A. F. Sheehan, J. S. Nakai, S. Y. Schwartz, K. Mochizuki, M. K. Savage, L. M. Wallace, S. A. Henrys, S. C. Webb, Y. Ito, R. E. Abercrombie, B. Fry, H. Shaddox, and E. K. Todd (2019), Seismicity at the Northern Hikurangi Margin, New Zealand, and Investigation of the Potential Spatial and Temporal Relationships With a Shallow Slow Slip Event, Journal of Geophysical Research: Solid Earth, 124(5), 4751-4766, doi: 10.1029/2018JB017211.

Yilmaz, Ö. (2001), Seismic Data Analysis, Investigations in Geophysics, 159-270 pp., Society of Exploration Geophysicists, doi:doi:10.1190/1.9781560801580.ch2.

Zal, H. J., K. Jacobs, M. K. Savage, J. Yarce, S. Mroczek, K. Graham, E. K. Todd, J. Nakai, Y. Iwasaki, A. Sheehan, K. Mochizuki, L. Wallace, S. Schwartz, S. Webb, and S. Henrys (2020), Temporal and spatial variations in seismic anisotropy and VP/VS ratios in a region of slow slip, Earth and Planetary Science Letters, 532, 115,970, doi:10.1016/j.epsl. 2019.115970 .

Zelt, C. A., and R. Ellis (1988), PRACTICAL AND EFFICIENT RAY TRACING IN TWODIMENSIONAL MEDIA FOR RAPID TRAVELTIME AND AMPLITUDE FORWARD MODELLING, Canadian Journal of Exploration Geophysics, 24(1), 16-31.

Zelt, C. A., and R. B. Smith (1992), Seismic traveltime inversion for 2-D crustal velocity structure, Geophysical Journal International, 108(1), 16-34, doi:10.1111/j.1365-246X. 1992.tb00836.x.

Zha, C.-S., T. S. Duffy, R. T. Downs, H.-K. Mao, and R. J. Hemley (1996), Sound velocity and elasticity of single-crystal forsterite to $16 \mathrm{GPa}$, Journal of Geophysical Research: Solid Earth, 101(B8), 17,535-17,545, doi:10.1029/96jb01266.

Zhang, H., and C. Thurber (2006), Development and Applications of Double-difference Seismic Tomography, pure and applied geophysics, 163(2), 373-403, doi:10.1007/ s00024-005-0021-y.

Zhu, J., J. P. Canales, S. Han, S. M. Carbotte, A. Arnulf, and M. R. Nedimović (2020), 
Vp/Vs Ratio of Incoming Sediments Off Cascadia Subduction Zone From Analysis of Controlled-Source Multicomponent OBS Records, Journal of Geophysical Research: Solid Earth, 125(6), 1-21, doi:10.1029/2019JB019239. 
